\title{
Aryl to Aryl Palladium Migration in the Heck and Suzuki Coupling of o-Halobiaryls \\ Supporting Information
}

Marino A. Campo, Haiming Zhang, Tuanli Yao, Abdellatif Ibdah, Ryan D. McCulla, Qinhua Huang, Jian Zhao, William S. Jenks* and Richard C. Larock*

Department of Chemistry, Iowa State University, Ames, Iowa 50011

wsjenks@iastate.eduandlarock@iastate.edu

\section{Table of Contents}

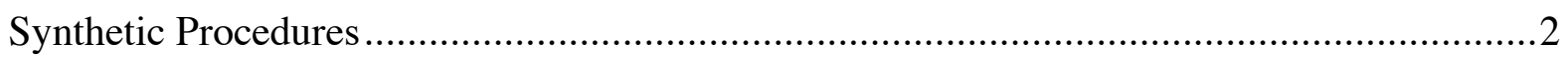

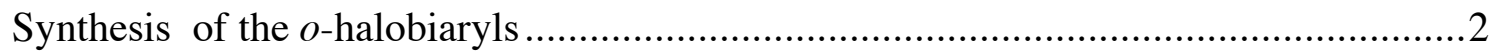

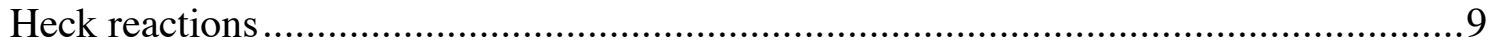

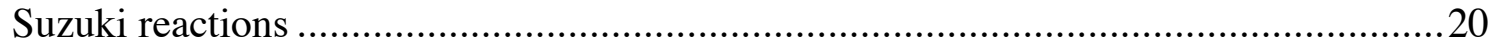

Mass Spectral Data for Deuteration Experiments..............................................................22

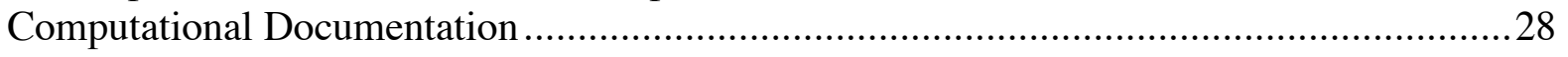

A simplified computational model, based on naked anions ........................................28

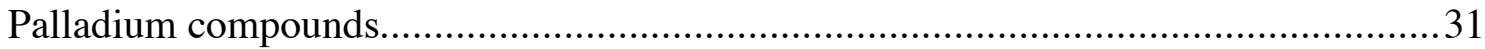

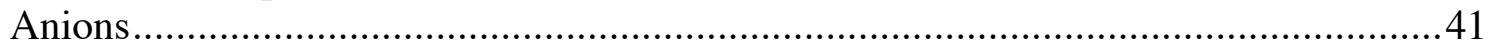

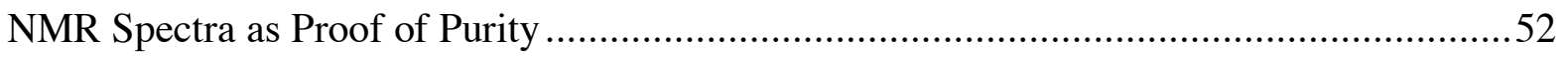

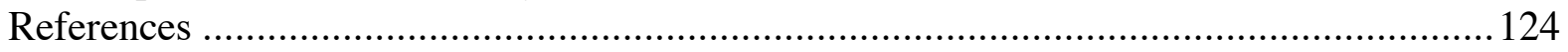




\section{Synthetic Procedures}

General procedures. All ${ }^{1} \mathrm{H}$ and ${ }^{13} \mathrm{C}$ NMR spectra were recorded at 300 and 75.5 MHz or 400 and $100 \mathrm{MHz}$ respectively. Thin-layer chromatography was performed using commercially prepared 60-mesh silica gel plates (Whatman K6F), and visualization was effected with short wavelength UV light $(254 \mathrm{~nm})$ and a basic $\mathrm{KMnO}_{4}$ solution [3 $\mathrm{g}$ of $\mathrm{KMnO}_{4}+20 \mathrm{~g}$ of $\mathrm{K}_{2} \mathrm{CO}_{3}+5 \mathrm{~mL}$ of $\mathrm{NaOH}(5 \%)+300 \mathrm{~mL}$ of $\left.\mathrm{H}_{2} \mathrm{O}\right]$. All melting points are uncorrected. High resolution mass spectra were recorded on a Kratos MS50TC double focusing magnetic sector mass spectrometer using EI at $70 \mathrm{eV}$.

Reagents. All reagents were used directly as obtained commercially unless otherwise noted. Anhydrous forms of THF, DME, DMF, diethyl ether, ethyl acetate and hexanes were purchased from Fisher Scientific Co. Tetra- $n$-butylammonium chloride (TBAC) was purchased from Lancaster Synthesis, Inc. 3-Bromoanisole, 1-bromo-2-iodobenzene, diphenylacetylene and ethyl acrylate were purchased from Aldrich Chemical Co., Inc. Cesium pivalate was prepared according to the procedure of Larock and Campo. ${ }^{1}$

Compounds $1 \mathbf{a},{ }^{1} 2 \mathbf{2 a},{ }^{2} 3 \mathbf{a},{ }^{2} \mathbf{1 c},{ }^{1} \mathbf{2 c},{ }^{2} \mathbf{3 c},{ }^{2} 4 \mathbf{a},{ }^{2} 4 \mathbf{c},{ }^{2} 5,{ }^{2} \mathbf{6},{ }^{2} 7,{ }^{2} \mathbf{8},{ }^{1} 9,{ }^{1}$ and $19^{1}$ have been reported previously.

\section{Synthesis of the $o$-halobiaryls}

$N, N$-Dimethyl-4-(2-iodophenyl)aniline (1b). Compound $1 \mathrm{~b}$ was prepared in two steps from compound 1e. To a solution of $1 \mathrm{e}(0.389 \mathrm{~g}, 1.19 \mathrm{mmol}), \mathrm{FeCl}_{3} \bullet 6 \mathrm{H}_{2} \mathrm{O}(5.0 \mathrm{mg}$, $0.018 \mathrm{mmol})$, and activated charcoal $(2.4 \mathrm{mg})$ in methanol $(10 \mathrm{~mL})$ under reflux was added $\mathrm{N}_{2} \mathrm{H}_{4} \bullet 1 \mathrm{H}_{2} \mathrm{O}(0.115 \mathrm{~g}, 3.03 \mathrm{mmol})$ dropwise over $10 \mathrm{~min}$. The mixture was refluxed for an 
additional $14 \mathrm{~h}$ and then allowed to cool to room temperature. The reaction mixture was diluted with diethyl ether $(50 \mathrm{~mL})$ and the organic layer was washed with water $(50 \mathrm{~mL})$. The organic layer was dried $\left(\mathrm{Na}_{2} \mathrm{SO}_{4}\right)$, filtered and the solvent evaporated under reduced pressure to obtain a yellow oil [presumed to be 4-(2-iodophenyl)aniline], which without further purification or characterization was dissolved in acetonitrile $(8 \mathrm{~mL})$. To this acetonitrile solution was added $37 \%$ aq formaldehyde $(0.96 \mathrm{~mL})$ and $\mathrm{NaBH}_{3} \mathrm{CN}(0.230 \mathrm{~g}$, $3.66 \mathrm{mmol}$ ). The resulting reaction mixture was stirred at room temperature while glacial acetic acid $(0.12 \mathrm{~mL})$ was added dropwise over $10 \mathrm{~min}$. After the addition, the reaction mixture was stirred for $40 \mathrm{~min}$, then another portion of glacial acetic acid $(0.12 \mathrm{~mL})$ was added at once and stirring continued for an additional $30 \mathrm{~min}$. The reaction mixture was diluted with diethyl ether $(50 \mathrm{~mL})$ and washed with $5 \%$ aq $\mathrm{NaOH}$. The organic layer was dried $\left(\mathrm{Na}_{2} \mathrm{SO}_{4}\right)$, filtered, and the solvent evaporated under reduced pressure. The residue was purified by chromatography on a silica gel column using 9:1 hexanes/ethyl acetate to afford $36.9 \mathrm{mg}(96 \%)$ of the indicated compound $\mathbf{1 b}$ as a colorless oil: ${ }^{1} \mathrm{H}$ NMR $\left(\mathrm{CDCl}_{3}\right) \delta 3.00(\mathrm{~s}$, $6 \mathrm{H}), 6.75-6.77(\mathrm{~m}, 2 \mathrm{H}), 6.94-6.98(\mathrm{~m}, 1 \mathrm{H}), 7.23-7.26(\mathrm{~m}, 2 \mathrm{H}), 7.29-7.36(\mathrm{~m}, 2 \mathrm{H}), 7.93(\mathrm{~d}, J$ $=8.0 \mathrm{~Hz}, 1 \mathrm{H}) ;{ }^{13} \mathrm{C} \mathrm{NMR}\left(\mathrm{CDCl}_{3}\right) \delta 40.5,99.6,111.5,128.1,128.2,130.1,130.3,132.4$, 139.5, 146.8, 149.9; IR $\left(\mathrm{CH}_{2} \mathrm{Cl}_{2}\right) 2917,2849,1612,1525,1461,1351 \mathrm{~cm}^{-1} ; \mathrm{HRMS} \mathrm{m/z}$ 323.0176 (calcd for $\mathrm{C}_{14} \mathrm{H}_{14} \mathrm{IN}, 323.0171$ ).

Ethyl 4-(2-iodophenyl)benzoate (1d). To a solution of 4-(2iodophenyl)benzaldehyde ${ }^{1}(0.33 \mathrm{~g}, 1.07 \mathrm{mmol})$ in ethanol $(10 \mathrm{~mL})$ was added activated $\mathrm{MnO}_{2}(1.90 \mathrm{~g}, 20$ equiv), $\mathrm{KCN}(0.35 \mathrm{~g}, 5$ equiv) and $\mathrm{HOAc}(0.20 \mathrm{~mL}, 3$ equiv). The mixture was stirred at room temperature for $1 \mathrm{~d}$ and filtered. The organic solution was diluted with EtOAc and washed with brine, dried over anhydrous $\mathrm{Na}_{2} \mathrm{SO}_{4}$, filtered and evaporated. The 
residue was purified on a silica gel column using 9:1 hexanes/EtOAc to afford 0.353 g (94\%) of the desired compound as a colorless oil: ${ }^{1} \mathrm{H} \mathrm{NMR}\left(\mathrm{CDCl}_{3}\right) \delta 1.41(\mathrm{t}, J=7.2 \mathrm{~Hz}, 3 \mathrm{H}), 4.40$ (q, $J=7.2 \mathrm{~Hz}, 2 \mathrm{H}), 7.04(\mathrm{dt}, J=1.8,7.8 \mathrm{~Hz}, 1 \mathrm{H}), 7.28(\mathrm{dd}, J=1.8,7.8 \mathrm{~Hz}, 1 \mathrm{H}), 7.37-7.44$ (m, 3H), $7.95(\mathrm{dd}, J=0.9,7.8 \mathrm{~Hz}, 1 \mathrm{H}), 8.11(\mathrm{dt}, J=8.4,1.8 \mathrm{~Hz}, 2 \mathrm{H}) ;{ }^{13} \mathrm{C} \mathrm{NMR}\left(\mathrm{CDCl}_{3}\right) \delta$ 14.7, 61.3, 98.1, 128.5, 129.5 (2), 129.6, 129.9, 130.1, 139.9, 145.9, 148.7, 166.6; IR (neat, $\mathrm{cm}^{-1}$ ) 3042, 2970, 1726; HRMS calcd for $\mathrm{C}_{15} \mathrm{H}_{13} \mathrm{IO}_{2}$ : 351.9960. Found: 351.9966 .

2-Iodo-4'-nitrobiphenyl (1e). Compound 1e was prepared by the procedure of Ozasa et al. ${ }^{3}$

$N, N$-Dimethyl-3-iodo-4-phenylaniline (4b). Compound $\mathbf{4 b}$ was prepared in two steps from 2-iodo-4-nitrobiphenyl (4e) by a procedure similar to that used for $\mathbf{1 d}$. The reaction mixture was purified by chromatography on a silica gel column using 9:1 hexanes/ethyl acetate to afford $38.1 \mathrm{mg}(98 \%)$ of the indicated compound (4b) as a yellow solid: $\operatorname{mp~76-77~}{ }^{\circ} \mathrm{C} ;{ }^{1} \mathrm{H} \mathrm{NMR}\left(\mathrm{CDCl}_{3}\right) \delta 2.96(\mathrm{~s}, 6 \mathrm{H}), 6.75(\mathrm{dd}, J=8.4,2.8 \mathrm{~Hz}, 1 \mathrm{H}), 7.14(\mathrm{~d}$, $J=8.4 \mathrm{~Hz}, 1 \mathrm{H}), 7.26(\mathrm{~d}, J=2.8 \mathrm{~Hz}, 1 \mathrm{H}), 7.33-7.38(\mathrm{~m}, 5 \mathrm{H}) ;{ }^{13} \mathrm{C} \mathrm{NMR}\left(\mathrm{CDCl}_{3}\right) \delta 40.5,99.5$, 112.3, 122.9, 127.0, 127.8, 129.8, 130.1, 134.4, 144.3, 150.4; IR $\left(\mathrm{CH}_{2} \mathrm{Cl}_{2}\right) 2917,2849,1598$,

Ethyl 3-iodo-4-phenylbenzoate (4d). Compound 4d was prepared from ethyl 4iodo-3-nitrobenzoate. ${ }^{4}$ First, a Suzuki coupling of ethyl 4-iodo-3-nitrobenzoate and $\mathrm{NaBPh}_{4}$ was carried out as follows. $\mathrm{NaBPh}_{4}(1.69 \mathrm{~g}, 4.94 \mathrm{mmol})$, ethyl 4-iodo-3-nitrobenzoate (1.38 g, $4.3 \mathrm{mmol}), \mathrm{LiCl}(0.182 \mathrm{~g}, 4.3 \mathrm{mmol}), \mathrm{Pd}(\mathrm{OAc})_{2}(0.048 \mathrm{~g}, 5 \mathrm{~mol} \%)$, and $\mathrm{PPh}_{3}(0.113 \mathrm{~g}, 10$ mol \%) in DMF $(10 \mathrm{~mL})$ were stirred under $\operatorname{Ar}$ at $70{ }^{\circ} \mathrm{C}$ for $2 \mathrm{~d}$. The reaction mixture was diluted with ether and washed with brine. The organic layer was dried $\left(\mathrm{MgSO}_{4}\right)$, filtered and evaporated to afford a yellow oil, which is presumed to be crude ethyl 3-nitro-4phenylbenzoate. The crude ethyl 3-nitro-4-phenylbenzoate was dissolved in a mixture of 
DME/EtOH/AcOH (5:4:1, $50 \mathrm{~mL})$ and $\mathrm{SnCl}_{2}(5.71 \mathrm{~g}, 30.1 \mathrm{mmol})$ was added. The resulting mixture was stirred at $60{ }^{\circ} \mathrm{C}$ under Ar for $16 \mathrm{~h}$, diluted with ether and washed with $10 \%$ aq $\mathrm{Na}_{2} \mathrm{CO}_{3}$. The organic layer was dried $\left(\mathrm{Na}_{2} \mathrm{SO}_{4}\right)$, filtered and evaporated. The residue was purified on a silica gel column using 3:1 hexanes/EtOAc to afford $0.93 \mathrm{~g} \mathrm{(90 \% )} \mathrm{of} \mathrm{ethyl} \mathrm{3-}$ amino-4-phenylbenzoate as a yellow oil, which was quickly diazotized and iodinated by the following procedure. To a solution of ethyl 3-amino-4-phenylbenzoate $(0.93 \mathrm{~g}, 3.86 \mathrm{mmol})$ in DME $(10 \mathrm{~mL})$ was added $\mathrm{H}_{2} \mathrm{O}(8 \mathrm{~mL})$ containing concentrated $\mathrm{H}_{2} \mathrm{SO}_{4}(0.7 \mathrm{~mL})$. The reaction mixture was stirred at $0{ }^{\circ} \mathrm{C}$ and $\mathrm{NaNO}_{2}(0.39 \mathrm{~g}, 5.6 \mathrm{mmol})$ in $\mathrm{H}_{2} \mathrm{O}(2 \mathrm{~mL})$ was added dropwise over $30 \mathrm{~min}$. The mixture was stirred for another $20 \mathrm{~min}$ at $0{ }^{\circ} \mathrm{C}$ and then the mixture was added to $\mathrm{NaI}(3.0 \mathrm{~g}, 20.0 \mathrm{mmol})$ in $\mathrm{H}_{2} \mathrm{O}(7 \mathrm{~mL})$. The mixture was extracted with ether and washed with $10 \%$ aq $\mathrm{Na}_{2} \mathrm{~S}_{2} \mathrm{O}_{3}$. The organic layer was filtered, dried $\left(\mathrm{Na}_{2} \mathrm{SO}_{4}\right)$ and evaporated. The residue was purified on a silica gel column using 7:1 hexanes/EtOAc to afford $1.06 \mathrm{~g}(78 \%)$ of compound $4 \mathbf{d}$ as a white solid: $\mathrm{mp} 76-77^{\circ} \mathrm{C} ;{ }^{1} \mathrm{H} \mathrm{NMR}\left(\mathrm{CDCl}_{3}\right) \delta$ $1.41(\mathrm{t}, J=7.2 \mathrm{~Hz}, 3 \mathrm{H}), 4.40(\mathrm{q}, J=7.2 \mathrm{~Hz}, 2 \mathrm{H}), 7.33-7.37(\mathrm{~m}, 3 \mathrm{H}), 7.41-7.45(\mathrm{~m}, 3 \mathrm{H}), 8.04$ $(\mathrm{dd}, J=8.0,1.6 \mathrm{~Hz}, 1 \mathrm{H}), 8.61(\mathrm{~d}, J=1.6 \mathrm{~Hz}, 1 \mathrm{H}) ;{ }^{13} \mathrm{C} \mathrm{NMR}\left(\mathrm{CDCl}_{3}\right) \delta$ 14.4, 61.4, 98.1, 128.2 (2), 129.0, 129.2, 129.9, 130.8, 140.6, 143.4, 150.9, 165.0; IR (neat, $\mathrm{cm}^{-1}$ ) 2973, 2818, 1722; HRMS calcd for $\mathrm{C}_{15} \mathrm{H}_{13} \mathrm{IO}_{2}$ : 351.9960. Found: 351.9966. Anal. calcd for $\mathrm{C}_{15} \mathrm{H}_{13} \mathrm{IO}_{2}$ : C, 51.14; H, 3.34. Found: C, 51.16; H, 3.72.

2-Iodo-4-nitrobiphenyl (4e). Compound 4e was prepared in two steps from 2-iodo4-nitroaniline. ${ }^{5}$ First of all, 2-iodo-4-nitroaniline was diazotized by the following procedure. To a solution of 2-iodo-4-nitroaniline (1.09 g, $4.13 \mathrm{mmol})$, and 48-50\% aq $\mathrm{HBF}_{4}(3.0 \mathrm{~g})$ in methanol $(20 \mathrm{~mL})$ was added $\mathrm{NaNO}_{2}(0.342 \mathrm{~g}, 4.96 \mathrm{mmol})$ in $\mathrm{H}_{2} \mathrm{O}(0.5 \mathrm{~mL})$ at room temperature. The reaction mixture was stirred for $30 \mathrm{~min}$, and then the reaction mixture was 
concentrated to $10 \mathrm{~mL}$ by passing a stream of Ar over it. At this point, a yellow solid had precipitated out of solution, and further crystallization was induced by cooling the mixture to $-20{ }^{\circ} \mathrm{C}$. The solid was filtered and washed repeatedly with diethyl ether. Recrystallization from methanol/diethyl ether afforded $0.673 \mathrm{~g}(49 \%)$ of 4-nitro-2-iodophenyldiazonium tetrafluoroborate as a yellow solid. 4-Nitro-2-iodophenyldiazonium tetrafluoroborate (82.7 $\mathrm{mg}, 0.25 \mathrm{mmol})$ was dissolved in dry DMSO $(2.0 \mathrm{~mL})$, then diluted with dry benzene $(8.0$ $\mathrm{mL}$ ). The reaction mixture was stirred at $80{ }^{\circ} \mathrm{C}$ for $2 \mathrm{~h}$ under Ar. The reaction mixture was diluted with diethyl ether $(20 \mathrm{~mL})$ and washed with brine $(30 \mathrm{~mL})$. The organic layer was dried $\left(\mathrm{Na}_{2} \mathrm{SO}_{4}\right)$, filtered and the solvent removed under reduced pressure. The residue was purified by chromatography on a silica gel column using 12:1 hexanes/ethyl acetate to obtain $35.8 \mathrm{mg}(44 \%)$ of the indicated compound $4 \mathbf{e}$ as a yellow solid: $\mathrm{mp} 91-92{ }^{\circ} \mathrm{C} ;{ }^{1} \mathrm{H} \mathrm{NMR}$ $\left(\mathrm{CDCl}_{3}\right) \delta 7.33-7.35(\mathrm{~m}, 2 \mathrm{H}), 7.45-7.48(\mathrm{~m}, 4 \mathrm{H}), 8.25(\mathrm{dd}, J=8.4,2.4 \mathrm{~Hz}, 1 \mathrm{H}), 8.80(\mathrm{~d}, J=$ $2.4 \mathrm{~Hz}, 1 \mathrm{H}) ;{ }^{13} \mathrm{C} \mathrm{NMR}\left(\mathrm{CDCl}_{3}\right) \delta 97.9,123.1,128.4,128.8,130.2,134.5,142.3,147.0,153.1$ (one $\mathrm{sp}^{2}$ carbon missing due to overlap); IR $\left(\mathrm{CH}_{2} \mathrm{Cl}_{2}\right)$ 2918, 2850, 1523, $1349 \mathrm{~cm}^{-1}$; HRMS $m / z 324.9606$ (calcd for $\mathrm{C}_{12} \mathrm{H}_{8} \mathrm{INO}_{2}, 324.9600$ ); Anal. C, 44.29, H, 2.29; N, 4.11 (calcd for $\left.\mathrm{C}_{12} \mathrm{H}_{8} \mathrm{INO}_{2}: \mathrm{C}, 44.33 ; \mathrm{H}, 2.48 ; \mathrm{N}, 4.34\right)$.

3-(2-Iodophenyl)-1-methylindole (12). Compound 12 was prepared in two steps from 2-(2-iodophenyl)acetaldehyde. ${ }^{6}$ A solution of 2-(2-iodophenyl)acetaldehyde (0.216 g, $0.88 \mathrm{mmol})$, phenylhydrazine $(0.105 \mathrm{~g}, 0.97 \mathrm{mmol})$ and methanesulfonic acid $(17 \mathrm{mg}, 0.18$ mmol) in ethanol $(5 \mathrm{~mL})$ was stirred at room temperature for 40 min under Ar. Then more methanesulfonic acid $(0.152 \mathrm{~g}, 1.58 \mathrm{mmol})$ was added and the mixture was stirred at $85{ }^{\circ} \mathrm{C}$ for $1.5 \mathrm{~d}$ under Ar. The reaction mixture was diluted with ether, washed with satd aq $\mathrm{NH}_{4} \mathrm{Cl}$ and the organic layer was dried $\left(\mathrm{Na}_{2} \mathrm{SO}_{4}\right)$, filtered and evaporated. The residue was purified 
on a silica gel column using 3:1 hexanes/EtOAc to afford $0.115 \mathrm{~g}(41 \%)$ of 3-(2iodophenyl)indole as a yellow oil: ${ }^{1} \mathrm{H} \mathrm{NMR}\left(\mathrm{CDCl}_{3}\right) \delta 6.99(\mathrm{~m}, 1 \mathrm{H}), 7.13-7.17(\mathrm{~m}, 1 \mathrm{H})$, $7.21-7.25(\mathrm{~m}, 1 \mathrm{H}), 7.32-7.47(\mathrm{~m}, 4 \mathrm{H}), 7.53(\mathrm{~d}, J=8.0 \mathrm{~Hz}, 1 \mathrm{H}), 8.00(\mathrm{dd}, J=8.0,0.8 \mathrm{~Hz}$, $1 \mathrm{H}), 8.18$ (br s, $1 \mathrm{H}) ;{ }^{13} \mathrm{C} \mathrm{NMR}\left(\mathrm{CDCl}_{3}\right) \delta 100.9,111.4,120.2,122.5,123.7,126.6,128.1$, $128.4,131.5,135.7,139.9,140.0$ (two $\mathrm{sp}^{2}$ carbons missing due to overlap). To a suspension of $\mathrm{NaH}(0.031 \mathrm{~g}, 1.3 \mathrm{mmol})$ in DMF $(2.0 \mathrm{~mL})$ at $0{ }^{\circ} \mathrm{C}$ was added 3-(2-iodophenyl)indole $(0.319 \mathrm{~g}, 1.0 \mathrm{mmol})$ in DMF $(3.0 \mathrm{~mL})$ and the mixture was stirred at room temperature for 30 min. $\mathrm{MeI}(1.42 \mathrm{~g}, 10.0 \mathrm{mmol})$ in DMF $(3 \mathrm{~mL})$ was added and the reaction mixture was stirred at room temperature for $14 \mathrm{~h}$. The reaction mixture was diluted with ether, washed with brine and the organic layer was dried $\left(\mathrm{MgSO}_{4}\right)$, filtered and evaporated. The residue was purified on a silica gel column using 5:1 hexanes/EtOAc to afford $0.320 \mathrm{~g}(96 \%)$ of compound 12 as a yellow oil: ${ }^{1} \mathrm{H}$ NMR $\left(\mathrm{CDCl}_{3}\right) \delta 3.84(\mathrm{~s}, 3 \mathrm{H}), 6.97-7.01(\mathrm{~m}, 1 \mathrm{H})$, 7.12-7.16 $(\mathrm{m}, 1 \mathrm{H}), 7.22-7.28(\mathrm{~m}, 2 \mathrm{H}), 7.35-7.40(\mathrm{~m}, 2 \mathrm{H}), 7.45(\mathrm{dd}, J=7.6,1.6 \mathrm{~Hz}, 1 \mathrm{H}), 7.53(\mathrm{~d}, J=$ 8.0 Hz, 1H), $7.99(\mathrm{dd}, J=7.6,0.8 \mathrm{~Hz}, 1 \mathrm{H}) ;{ }^{13} \mathrm{C} \mathrm{NMR}\left(\mathrm{CDCl}_{3}\right) \delta 33.1,100.8,109.5,118.5$, $119.7,120.3,122.0,127.0,128.1,128.2,128.4,131.5,136.6,139.9,140.1 ;$ IR (neat, $\mathrm{cm}^{-1}$ ) 3050, 2922, 1547; HRMS calcd for $\mathrm{C}_{15} \mathrm{H}_{12} \mathrm{IN}$ : 333.0014. Found: 333.0024 .

2-Iodo-3',5'-dimethylbiphenyl (13). 2-Iodo-3',5'-dimethylbiphenyl (13) was prepared by a procedure reported by Hart. $^{7}$ A solution of 2-bromoiodobenzene (1.415 g, 5.0 $\mathrm{mmol})$ in THF (10 $\mathrm{mL})$ was added slowly $(90 \mathrm{~min})$ to a solution of 3,5dimethylphenylmagnesium bromide [prepared from 1-bromo-3,5-dimethylbenzene (1.85 g, $10 \mathrm{mmol})$ and $\mathrm{Mg}(0.246 \mathrm{~g}, 10 \mathrm{mmol})$ in THF $(30 \mathrm{~mL})]$, and the mixture was stirred under Ar for an additional $14 \mathrm{~h}$ at room temperature. The reaction was quenched by adding $\mathrm{I}_{2}(3.8$ $\mathrm{g}, 15 \mathrm{mmol}$ ), and the mixture was stirred for an additional $30 \mathrm{~min}$ at room temperature. The 
excess $\mathrm{I}_{2}$ was destroyed by adding $10 \%$ aq $\mathrm{NaHSO}_{3}(35 \mathrm{~mL})$ and the organic layer was separated and rewashed with brine $(20 \mathrm{~mL})$. Finally, the organic layer was dried $\left(\mathrm{MgSO}_{4}\right)$, filtered, and the solvent removed under reduced pressure. The residue was chromatographed using hexanes to afford $0.836 \mathrm{~g}(54 \%)$ of the desired compound 13 as a clear oil: ${ }^{1} \mathrm{H}$ NMR $\left(\mathrm{CDCl}_{3}\right) \delta 2.36(\mathrm{~s}, 6 \mathrm{H}), 6.95-7.02(\mathrm{~m}, 4 \mathrm{H}), 7.33-7.82(\mathrm{~m}, 1 \mathrm{H}), 7.92-7.94(\mathrm{~m}, 1 \mathrm{H}) ;{ }^{13} \mathrm{C} \mathrm{NMR}$ $\left(\mathrm{CDCl}_{3}\right) \delta 21.6,98.9,127.3,128.3,128.8,129.4,130.3,137.6,139.6,144.3,147.1 ; \mathrm{IR}$ $\left(\mathrm{CH}_{2} \mathrm{Cl}_{2}\right)$ 2917, 2849, 1602, 1461, $1015 \mathrm{~cm}^{-1} ; \mathrm{HRMS} \mathrm{m} / z 308.0070$ (calcd for $\mathrm{C}_{14} \mathrm{H}_{13} \mathrm{I}$, 308.0062).

2-Iodo-2'-methylbiphenyl (16). Compound 16 was prepared by a procedure reported by Hart. ${ }^{7}$ A solution of 2-bromoiodobenzene $(1.415 \mathrm{~g}, 5.0 \mathrm{mmol})$ in THF (10 mL) was added slowly (90 min) to a solution of 2-methylphenylmagnesium bromide [prepared from 2-bromotoluene $(1.71 \mathrm{~g}, 10 \mathrm{mmol})$ and $\mathrm{Mg}(0.246 \mathrm{~g}, 10 \mathrm{mmol})$ in THF (30 mL)], and the mixture was stirred under Ar for an additional $14 \mathrm{~h}$ at room temperature. The reaction was quenched by adding $\mathrm{I}_{2}(3.8 \mathrm{~g}, 15 \mathrm{mmol})$, and the mixture was stirred for an additional 30 min at room temperature. The excess $\mathrm{I}_{2}$ was destroyed by adding $10 \%$ aq $\mathrm{NaHSO}_{3}(35 \mathrm{~mL})$ and the organic layer was separated and rewashed with brine $(20 \mathrm{~mL})$. Finally, the organic layer was dried $\left(\mathrm{MgSO}_{4}\right)$, filtered, and the solvent removed under reduced pressure. The residue was chromatographed using hexanes to afford $0.97 \mathrm{~g}(66 \%)$ of the desired compound 16 as a clear oil: ${ }^{1} \mathrm{H}$ NMR $\left(\mathrm{CDCl}_{3}\right) \delta 2.07(\mathrm{~s}, 3 \mathrm{H}), 7.01-7.07(\mathrm{~m}, 2 \mathrm{H}), 7.20-7.31(\mathrm{~m}, 4 \mathrm{H})$, 7.36-7.40 (m, 1H), 7.92-7.94 (m, 1H); ${ }^{13} \mathrm{C} \mathrm{NMR}\left(\mathrm{CDCl}_{3}\right) \delta 20.1,100.1,125.6,128.0,128.1$, 128.7, 129.2, 129.7, 129.9, 135.7, 138.9, 144.4, 146.8; IR $\left(\mathrm{CH}_{2} \mathrm{Cl}_{2}\right) 3056,3018,2921,1461$, $1428 \mathrm{~cm}^{-1}$; HRMS m/z, 293.9910 (calcd for $\mathrm{C}_{14} \mathrm{H}_{13} \mathrm{I}, 293.9906$ ). 
2-Iodo-3'-methoxybiphenyl (26). Compound 26 was prepared by a procedure reported by Hart. ${ }^{7}$ A solution of 2-bromoiodobenzene (1.415 g, $\left.5.0 \mathrm{mmol}\right)$ in THF (10 mL) was added slowly (90 min) to a solution of 3-methoxyphenylmagnesium bromide [prepared from 3-bromoanisole (1.87 g, $10 \mathrm{mmol})$ and $\mathrm{Mg}(0.246 \mathrm{~g}, 10 \mathrm{mmol})$ in THF (30 mL)], and the mixture was stirred under Ar for an additional $14 \mathrm{~h}$ at room temperature. The reaction was quenched by adding $\mathrm{I}_{2}(3.8 \mathrm{~g}, 15 \mathrm{mmol})$, and the mixture was stirred for an additional 30 min at room temperature. The excess $\mathrm{I}_{2}$ was destroyed by adding $10 \%$ aq $\mathrm{NaHSO}_{3}(35 \mathrm{~mL})$ and the organic layer was separated and rewashed with brine $(20 \mathrm{~mL})$. Finally, the organic layer was dried $\left(\mathrm{MgSO}_{4}\right)$, filtered, and the solvent removed under reduced pressure. The residue was chromatographed using 30:1 hexanes/ethyl acetate to afford $0.620 \mathrm{~g} \mathrm{(40 \% )}$ of the desired compound 26 as a clear oil: ${ }^{1} \mathrm{H}$ NMR $\left(\mathrm{CDCl}_{3}\right) \delta 3.84(\mathrm{~s}, 3 \mathrm{H}), 6.88-6.95(\mathrm{~m}, 3 \mathrm{H})$, 7.01-7.05 (m, 1H), 7.29-7.38 (m, 3H), $7.95(\mathrm{dd}, J=8.0,1.2 \mathrm{~Hz}, 1 \mathrm{H}) ;{ }^{13} \mathrm{C} \mathrm{NMR}\left(\mathrm{CDCl}_{3}\right) \delta$ $55.4,98.5,113.4,115.0,121.8,128.1,128.9,129.1,130.1,139.6,145.5,146.5,159.1$; HRMS $m / z 309.9859$ (calcd for $\mathrm{C}_{13} \mathrm{H}_{11} \mathrm{IO}, 309.9855$ ).

\section{Heck reactions}

The following procedures are representative of the palladium-catalyzed Heck reactions with ethyl acrylate.

Migration reaction conditions (procedure $\mathbf{C})$ : The appropriate aryl halide $(0.25$ mmol), $\mathrm{Pd}(\mathrm{OAc})_{2}(2.8 \mathrm{mg}, 0.0125 \mathrm{mmol}), 1$,1-bis(diphenylphosphino)methane (dppm) (4.8 $\mathrm{mg}, 0.0125 \mathrm{mmol}), \mathrm{CsO}_{2} \mathrm{CCMe}_{3}$ (CsPiv) $(0.117 \mathrm{~g}, 0.5 \mathrm{mmol})$ and ethyl acrylate $(0.025 \mathrm{~g}$, $0.25 \mathrm{mmol})$ in $\mathrm{DMF}(4.0 \mathrm{ml})$ under $\mathrm{Ar}$ at $100{ }^{\circ} \mathrm{C}$ were stirred for the specified length of time. The reaction mixture was then cooled to room temperature, diluted with diethyl ether (35 
$\mathrm{mL})$ and washed with brine $(30 \mathrm{~mL})$. The aqueous layer was reextracted with diethyl ether $(15 \mathrm{~mL})$. The organic layers were combined, dried $\left(\mathrm{MgSO}_{4}\right)$, filtered, and the solvent removed under reduced pressure. The residue was purified by column chromatography on a silica gel column.

Migration reaction conditions (procedure D): The appropriate aryl halide $(0.25$ $\mathrm{mmol}), \mathrm{Pd}(\mathrm{OAc})_{2}(2.8 \mathrm{mg}, 0.0125 \mathrm{mmol}), 1,1$-bis(diphenylphosphino)methane (dppm) (4.8 $\mathrm{mg}, 0.0125 \mathrm{mmol}), \mathrm{CsO}_{2} \mathrm{CCMe}_{3}(\mathrm{CsPiv})(0.117 \mathrm{~g}, 0.5 \mathrm{mmol})$ and ethyl acrylate $(0.025 \mathrm{~g}$, $0.25 \mathrm{mmol})$ in wet DMF $\left(5 \% \mathrm{H}_{2} \mathrm{O}\right.$ by volume) $(4.0 \mathrm{~mL})$ under $\mathrm{Ar}$ at $100{ }^{\circ} \mathrm{C}$ were stirred for the specified length of time. The reaction mixture was then cooled to room temperature, diluted with diethyl ether $(35 \mathrm{~mL})$ and washed with brine $(30 \mathrm{~mL})$. The aqueous layer was reextracted with diethyl ether $(15 \mathrm{~mL})$. The organic layers were combined, dried $\left(\mathrm{MgSO}_{4}\right)$, filtered, and the solvent removed under reduced pressure. The residue was purified by column chromatography on a silica gel column.

Non-migration reaction conditions (procedure A): ${ }^{6}$ The appropriate aryl iodide (0.25 mmol), $\mathrm{Pd}(\mathrm{OAc})_{2}(2.8 \mathrm{mg}, 0.0125 \mathrm{mmol}), n-\mathrm{Bu}_{4} \mathrm{NCl}(0.0694 \mathrm{~g}, 0.25 \mathrm{mmol}), \mathrm{NaHCO}_{3}$ $(0.042 \mathrm{~g}, 0.5 \mathrm{mmol})$ and ethyl acrylate $(0.10 \mathrm{~g}, 1.0 \mathrm{mmol})$ in DMF $(1 \mathrm{ml})$ under Ar at $100{ }^{\circ} \mathrm{C}$ were stirred for the specified length of time. The reaction mixture was then cooled to room temperature, diluted with diethyl ether $(35 \mathrm{~mL})$ and washed with brine $(30 \mathrm{~mL})$. The aqueous layer was reextracted with diethyl ether $(15 \mathrm{~mL})$. The organic layers were combined, dried $\left(\mathrm{MgSO}_{4}\right)$, filtered, and the solvent removed under reduced pressure. The residue was purified by column chromatography on a silica gel column.

Non-migration reaction conditions (procedure B): The appropriate aryl iodide (0.25 mmol), $\mathrm{Pd}(\mathrm{OAc})_{2}(2.8 \mathrm{mg}, 0.0125 \mathrm{mmol}), n-\mathrm{Bu}_{4} \mathrm{NCl}(0.0694 \mathrm{~g}, 0.25 \mathrm{mmol}), \mathrm{Et}_{3} \mathrm{~N}(50.5$ 
$\mathrm{mg}, 0.5 \mathrm{mmol})$ and ethyl acrylate $(0.10 \mathrm{~g}, 1.0 \mathrm{mmol})$ in DMF $(1 \mathrm{ml})$ under Ar at $100{ }^{\circ} \mathrm{C}$ were stirred for the specified length of time. The reaction mixture was then cooled to room temperature, diluted with diethyl ether $(35 \mathrm{~mL})$ and washed with brine $(30 \mathrm{~mL})$. The aqueous layer was reextracted with diethyl ether $(15 \mathrm{~mL})$. The organic layers were combined, dried $\left(\mathrm{MgSO}_{4}\right)$, filtered, and the solvent removed under reduced pressure. The residue was purified by column chromatography on a silica gel column.

Ethyl $\boldsymbol{E}$-3-(4'-dimethylaminobiphen-2-yl)acrylate (2b). $N, N$-Dimethyl-4-(2iodophenyl)aniline (1b) $(80.8 \mathrm{mg}, 0.25 \mathrm{mmol})$ was allowed to react with ethyl acrylate under non-migration reaction conditions (procedure $\mathrm{A}$ ) for $1 \mathrm{~d}$. The reaction mixture was chromatographed using 5:1 hexanes/ethyl acetate to afford $59.0 \mathrm{mg}(80 \%)$ of the indicated compound as a yellow solid: $\mathrm{mp} 117-118{ }^{\circ} \mathrm{C} ;{ }^{1} \mathrm{H}$ NMR $\left(\mathrm{CDCl}_{3}\right) \delta 1.30(\mathrm{t}, J=7.0 \mathrm{~Hz}, 3 \mathrm{H})$, $3.01(\mathrm{~s}, 6 \mathrm{H}), 4.22(\mathrm{q}, J=7.2 \mathrm{~Hz}, 2 \mathrm{H}), 6.39(\mathrm{~d}, J=15.6 \mathrm{~Hz}, 1 \mathrm{H}), 6.78-6.80(\mathrm{~m}, 2 \mathrm{H}), 7.20-7.22$ $(\mathrm{m}, 2 \mathrm{H}), 7.31-7.40(\mathrm{~m}, 3 \mathrm{H}), 7.66(\mathrm{~d}, J=7.2 \mathrm{~Hz}, 1 \mathrm{H}), 7.84(\mathrm{~d}, J=16.0 \mathrm{~Hz}, 1 \mathrm{H}) ;{ }^{13} \mathrm{C} \mathrm{NMR}$ $\left(\mathrm{CDCl}_{3}\right) \delta 14.4,40.6,60.3,112.2,118.5,126.7,126.9,127.7,129.8,130.4,130.7,132.6$, 143.2, 144.7, 150.0, 167.2; IR $\left(\mathrm{CH}_{2} \mathrm{Cl}_{2}\right) 2975,2926,2857,1715,1612,1631,1526 \mathrm{~cm}^{-1}$; HRMS m/z, 295.1580 (calcd for $\mathrm{C}_{19} \mathrm{H}_{21} \mathrm{NO}_{2}, 295.1572$ ); Anal. C, 77.05, H, 7.29; N, 4.57 (calcd for $\mathrm{C}_{19} \mathrm{H}_{21} \mathrm{NO}_{2}: \mathrm{C}, 77.26 ; \mathrm{H}, 7.17 ; \mathrm{N}, 4.77$ ).

Ethyl $\boldsymbol{E}$-3-(4-dimethylaminobiphen-2-yl)acrylate (3b). $N, N$-Dimethyl-3-iodo-4phenylaniline (4b) (80.8 $\mathrm{mg}, 0.25 \mathrm{mmol}$ ) was allowed to react with ethyl acrylate under nonmigration reaction conditions (procedure A) for $1 \mathrm{~d}$. The reaction mixture was chromatographed using 5:1 hexanes/ethyl acetate to afford $73.8 \mathrm{mg}(100 \%)$ of the indicated compound as a yellow oil: ${ }^{1} \mathrm{H} \mathrm{NMR}\left(\mathrm{CDCl}_{3}\right) \delta 1.28(\mathrm{t}, J=7.2 \mathrm{~Hz}, 3 \mathrm{H}), 3.01(\mathrm{~s}, 6 \mathrm{H}), 4.21(\mathrm{q}$, $J=7.2 \mathrm{~Hz}, 2 \mathrm{H}), 6.39(\mathrm{~d}, J=15.6 \mathrm{~Hz}, 1 \mathrm{H}), 6.85(\mathrm{dd}, J=8.8,2.8 \mathrm{~Hz}, 1 \mathrm{H}), 6.97(\mathrm{~d}, J=2.8 \mathrm{~Hz}$, 
1H), 7.24-7.34 (m, 4H), 7.38-7.42 (m, 2H), $7.77(\mathrm{~d}, J=16.0 \mathrm{~Hz}, 1 \mathrm{H}) ;{ }^{13} \mathrm{C} \mathrm{NMR}\left(\mathrm{CDCl}_{3}\right) \delta$ 14.4, 40.6, 60.4, 109.8, 114.7, 118.7, 126.8, 128.2, 130.0, 131.3, 131.6, 133.1, 140.2, 145.1, 149.8, 167.1; IR $\left(\mathrm{CH}_{2} \mathrm{Cl}_{2}\right)$ 2975, 2920, 1711, 1632, 1605, $1174 \mathrm{~cm}^{-1} ;$ HRMS $m / z 295.1577$ (calcd for $\mathrm{C}_{19} \mathrm{H}_{21} \mathrm{NO}_{2}, 295.1572$ ).

Ethyl $E$-3-(4'-dimethylaminobiphen-2-yl)acrylate (2b) and ethyl E-3-(4dimethylaminobiphen-2-yl)acrylate (3b). Compound $\mathbf{1 b}(80.8 \mathrm{mg}, 0.25 \mathrm{mmol})$ was allowed to react with ethyl acrylate under our migration reaction conditions (procedure $\mathrm{C}$ ) for $1 \mathrm{~d}$. The reaction mixture was chromatographed using 5:1 hexanes/ethyl acetate to afford $66.4 \mathrm{mg}(90 \%)$ of the indicated compounds as a clear oil in a 55:45 ratio. Similarly, compound $\mathbf{4 b}$ ( $80.8 \mathrm{mg}, 0.25 \mathrm{mmol})$ was allowed to react with ethyl acrylate under our standard migration reaction conditions A for $1 \mathrm{~d}$. The reaction mixture was chromatographed using 5:1 hexanes/ethyl acetate to afford $68.6 \mathrm{mg}(93 \%)$ of the indicated compounds as a clear oil in a 49:51 ratio.

Ethyl E-3-(4'-ethoxycarbonylbiphen-2-yl)acrylate (2d). Ethyl 4-(2iodophenyl)benzoate (1d) $(76.2 \mathrm{mg}, 0.25 \mathrm{mmol})$ was allowed to react for $2 \mathrm{~d}$ under nonmigration reaction conditions (procedure A) with ethyl acrylate. The reaction mixture was chromatographed using 7:1 hexanes/ethyl acetate to afford $58.3 \mathrm{mg}(72 \%)$ of the indicated compound (2d) as a clear oil: ${ }^{1} \mathrm{H}$ NMR $\left(\mathrm{CDCl}_{3}\right) \delta 1.28(\mathrm{t}, J=7.0 \mathrm{~Hz}, 3 \mathrm{H}), 1.42(\mathrm{t}, J=7.2$ $\mathrm{Hz}, 3 \mathrm{H}), 4.21(\mathrm{q}, J=7.2 \mathrm{~Hz}, 2 \mathrm{H}), 4.42(\mathrm{q}, J=7.2 \mathrm{~Hz}, 2 \mathrm{H}), 6.41(\mathrm{~d}, J=15.6 \mathrm{~Hz}, 1 \mathrm{H}), 7.36-$ $7.46(\mathrm{~m}, 5 \mathrm{H}), 7.66(\mathrm{~d}, J=16.0 \mathrm{~Hz}, 1 \mathrm{H}), 7.70-7.72(\mathrm{~m}, 1 \mathrm{H}), 8.11-8.13(\mathrm{~m}, 2 \mathrm{H}) ;{ }^{13} \mathrm{C} \mathrm{NMR}$ $\left(\mathrm{CDCl}_{3}\right) \delta 14.3,14.4,60.5,61.1,119.8,127.0,128.3,129.6,129.7,129.9,130.0,130.4$ 132.7, 141.8, 143.2, 144.5, 166.4, 166.7; IR $\left(\mathrm{CH}_{2} \mathrm{Cl}_{2}\right) 2880,2933,1713,1633 \mathrm{~cm}^{-1} ; \mathrm{HRMS}$ $m / z 324.1368$ (calcd for $\mathrm{C}_{20} \mathrm{H}_{20} \mathrm{O}_{4}, 324.1362$ ). 
Ethyl $E$-3-(4-ethoxycarbonylbiphen-2-yl)acrylate (3d). Ethyl 3-iodo-4phenylbenzoate (4d) $(76.2 \mathrm{mg}, 0.25 \mathrm{mmol})$ was allowed to react with ethyl acrylate under non-migration reaction conditions (procedure A) for $1 \mathrm{~d}$. The reaction mixture was chromatographed using 7:1 hexanes/ethyl acetate to afford $80.2 \mathrm{mg}(99 \%)$ of the indicated compound (3d) as a clear oil: ${ }^{1} \mathrm{H}$ NMR $\left(\mathrm{CDCl}_{3}\right) \delta 1.30(\mathrm{t}, J=7.2 \mathrm{~Hz}, 3 \mathrm{H}), 1.43(\mathrm{t}, J=7.0$ $\mathrm{Hz}, 3 \mathrm{H}), 4.22(\mathrm{q}, J=7.2 \mathrm{~Hz}, 2 \mathrm{H}), 4.42(\mathrm{q}, J=7.2 \mathrm{~Hz}, 2 \mathrm{H}), 6.52(\mathrm{~d}, J=16.0 \mathrm{~Hz}, 1 \mathrm{H}), 7.32-$ $7.33(\mathrm{~m}, 2 \mathrm{H}), 7.42-7.48(\mathrm{~m}, 4 \mathrm{H}), 7.72(\mathrm{~d}, J=16.0 \mathrm{~Hz}, 1 \mathrm{H}), 8.08-8.10(\mathrm{~m}, 1 \mathrm{H}), 8.38(\mathrm{~s}, 1 \mathrm{H})$;

${ }^{13} \mathrm{C} \mathrm{NMR}\left(\mathrm{CDCl}_{3}\right) \delta 14.3,14.4,60.6,61.3,120.4,128.2,128.5,129.7,129.9,130.5,130.7$, $132.9,139.0,142.8,146.9,166.0,166.7$ (one $\mathrm{sp}^{2}$ carbon missing due to overlap) ; IR $\left(\mathrm{CH}_{2} \mathrm{Cl}_{2}\right)$ 2980, 1717, 1636, 1285, $1245 \mathrm{~cm}^{-1}$; HRMS m/z 324.1368 (calcd for $\mathrm{C}_{20} \mathrm{H}_{20} \mathrm{O}_{4}$, 324.1362).

Ethyl E-3-(4'-ethoxycarbonylbiphen-2-yl)acrylate (2d) and ethyl $E$-3-(4ethoxycarbonylbiphen-2-yl)acrylate (3d). Compound 1d (76.2 mg, $0.25 \mathrm{mmol})$ was allowed to react with ethyl acrylate under our migration reaction conditions (procedure C) for $1 \mathrm{~d}$. The reaction mixture was chromatographed using 7:1 hexanes/ethyl acetate to afford $69.6 \mathrm{mg}(86 \%)$ of the indicated compounds as a clear oil in a 48:52 ratio. Similarly, compound $4 \mathbf{d}(76.2 \mathrm{mg}, 0.25 \mathrm{mmol})$ was allowed to react with ethyl acrylate under our migration reaction conditions (procedure $\mathrm{C}$ ) for $1 \mathrm{~d}$. The reaction mixture was chromatographed using 7:1 hexanes/ethyl acetate to afford $67.2 \mathrm{mg}(83 \%)$ of the indicated compounds as a clear oil in a 42:58 ratio.

Ethyl $\boldsymbol{E}$-3-(4'-nitrobiphen-2-yl)acrylate (2e). Compound 1e (81.2 mg, $0.25 \mathrm{mmol})$ was allowed to react with ethyl acrylate under non-migration reaction conditions (procedure B). After $1 \mathrm{~d}$, the reaction mixture was chromatographed using 5:1 hexanes/ethyl acetate to 
afford $63.1 \mathrm{mg}(85 \%)$ of the indicated compound $\mathbf{2 e}$ as a yellow oil: ${ }^{1} \mathrm{H} \mathrm{NMR}\left(\mathrm{CDCl}_{3}\right) \delta 1.29$ $(\mathrm{t}, J=7.0 \mathrm{~Hz}, 3 \mathrm{H}), 4.22(\mathrm{q}, J=7.2 \mathrm{~Hz}, 2 \mathrm{H}), 6.43(\mathrm{~d}, J=16.0 \mathrm{~Hz}, 1 \mathrm{H}), 7.36-7.38(\mathrm{~m}, 1 \mathrm{H})$, 7.46-7.51 (m, 4H), $7.60(\mathrm{~d}, J=15.6 \mathrm{~Hz}, 1 \mathrm{H}), 7.72-7.74(\mathrm{~m}, 1 \mathrm{H}), 8.29-8.32(\mathrm{~m}, 2 \mathrm{H}) ;{ }^{13} \mathrm{C}$ $\operatorname{NMR}\left(\mathrm{CDCl}_{3}\right) \delta 14.3,60.7,120.5,123.6,127.3,129.0,130.1,130.3,130.7,132.7,140.3$, 142.4, 146.7, 147.3, 166.6; IR $\left(\mathrm{CH}_{2} \mathrm{Cl}_{2}\right) 2917,1710,1517,1348,1314,1179 \mathrm{~cm}^{-1}$; HRMS $m / z 297.1010$ (calcd for $\mathrm{C}_{17} \mathrm{H}_{15} \mathrm{NO}_{4}, 297.1001$ ).

Ethyl $\boldsymbol{E}$-3-(4-nitrobiphen-2-yl)acrylate (3e). Compound 4e (81.2 mg, $0.25 \mathrm{mmol})$ was allowed to react with ethyl acrylate under non-migration reaction conditions (procedure B). After $1 \mathrm{~d}$, the reaction mixture was chromatographed using 5:1 hexanes/ethyl acetate to afford $66.1 \mathrm{mg}(89 \%)$ of the indicated compound $\mathbf{3 e}$ as a yellow solid: $\mathrm{mp}=118-119{ }^{\circ} \mathrm{C},{ }^{1} \mathrm{H}$ $\operatorname{NMR}\left(\mathrm{CDCl}_{3}\right) \delta 1.31(\mathrm{t}, J=7.2 \mathrm{~Hz}, 3 \mathrm{H}), 4.24(\mathrm{q}, J=7.2 \mathrm{~Hz}, 2 \mathrm{H}), 6.55(\mathrm{~d}, J=15.9 \mathrm{~Hz}, 1 \mathrm{H})$, 7.31-7.34 (m, 2H), 7.46-7.56 (m, 4H), $7.69(\mathrm{~d}, J=15.9 \mathrm{~Hz}, 1 \mathrm{H}), 8.26(\mathrm{dd}, J=8.4,2.4 \mathrm{~Hz}$, $1 \mathrm{H}), 8.55(\mathrm{~d}, J=2.4 \mathrm{~Hz}, 1 \mathrm{H}) ;{ }^{13} \mathrm{C} \mathrm{NMR}\left(\mathrm{CDCl}_{3}\right) \delta 14.5,61.0,122.2,122.2,124.2,128.9$, 129.1, 129.7, 131.8, 134.4, 138.1, 141.6, 147.5, 148.8, 166.3; IR $\left(\mathrm{CH}_{2} \mathrm{Cl}_{2}\right)$ 2974, 2917, 2849, 1719, 1523, 1347, 1179, $1118 \mathrm{~cm}^{-1}$; HRMS m/z 297.1010 (calcd for $\mathrm{C}_{17} \mathrm{H}_{15} \mathrm{NO}_{4}, 297.1001$ ); Anal. C, 68.41; H, 4.84; N, 4.51 (calcd for $\mathrm{C}_{17} \mathrm{H}_{15} \mathrm{NO}_{4}$ : C, 68.68; H, 5.09; N, 4.71).

\section{Ethyl $E$-3-(4'-nitrobiphen-2-yl)acrylate (2e) and ethyl $E$-3-(4-nitrobiphen-2-}

yl)acrylate (3e). Compound $1 \mathbf{e}(81.2 \mathrm{mg}, 0.25 \mathrm{mmol})$ was allowed to react with ethyl acrylate under our migration reaction conditions (procedure D) for $2.5 \mathrm{~d}$. The reaction mixture was chromatographed using 5:1 hexanes/ethyl acetate to afford $34.2 \mathrm{mg}(46 \%)$ of the indicated compounds as a yellow oil in a 39:61 ratio. In addition, we obtained $19.9 \mathrm{mg}$ (40\%) of 4-nitrobiphenyl as a yellow solid with spectral properties identical to those previously reported. ${ }^{11}$ Similarly, compound $4 \mathbf{e}(81.2 \mathrm{mg}, 0.25 \mathrm{mmol})$ was allowed to react 
with ethyl acrylate under our migration reaction conditions (procedure $\mathrm{D}$ ) for $2.5 \mathrm{~d}$. The reaction mixture was chromatographed using 5:1 hexanes/ethyl acetate to afford $27.5 \mathrm{mg}$ (37\%) of the indicated compounds as a yellow oil in a 33:67 ratio. In addition, we obtained $24.9 \mathrm{mg}(50 \%)$ of 4-nitrobiphenyl as a yellow solid with spectral properties identical to those previously reported. ${ }^{11}$

Ethyl $\boldsymbol{E}$-3-(3-phenylbenzofuran-2-yl)acrylate (6). Compound 5 (80.0 mg, 0.25 mmol) was allowed to react with ethyl acrylate under our migration reaction conditions (procedure C) for $1 \mathrm{~d}$. The reaction mixture was chromatographed using 7:1 hexanes/ethyl acetate to afford $62.1 \mathrm{mg}(85 \%)$ of the indicated compound as a white solid: $\mathrm{mp} 84-85^{\circ} \mathrm{C}$, ${ }^{1} \mathrm{H} \mathrm{NMR}\left(\mathrm{CDCl}_{3}\right) \delta 1.32(\mathrm{t}, J=7.2 \mathrm{~Hz}, 3 \mathrm{H}), 4.25(\mathrm{q}, J=7.2 \mathrm{~Hz}, 2 \mathrm{H}), 6.68(\mathrm{~d}, J=15.6 \mathrm{~Hz}$, 1H), 7.25-7.29 (m, 1H), 7.40-7.54 (m, 7H), 7.63-7.68 (m, 2H); ${ }^{13} \mathrm{C} \mathrm{NMR}\left(\mathrm{CDCl}_{3}\right)$ \& 14.4, $60.7,111.6,119.4,121.0,123.5,125.7,127.0,128.4,128.4,129.2,129.6,130.3,131.2$, 148.3, 155.0, 167.0; IR $\left(\mathrm{CH}_{2} \mathrm{Cl}_{2}\right)$ 3062, 2979, 1709, 1628, $1450 \mathrm{~cm}^{-1}$; HRMS m/z 292.1104 (calcd for $\left.\mathrm{C}_{19} \mathrm{H}_{16} \mathrm{O}_{3}, 292.1099\right)$. Similarly, compound 8 (80.0 mg, $\left.0.25 \mathrm{mmol}\right)$ was allowed to react with ethyl acrylate under our migration reaction conditions (procedure $\mathrm{C}$ ). The reaction mixture was chromatographed using 7:1 hexanes/ethyl acetate to afford $57.0 \mathrm{mg}(78 \%)$ of the indicated compound as a yellow solid: $\mathrm{mp} 82-84^{\circ} \mathrm{C}$.

Ethyl $\boldsymbol{E}$-3-[2-(benzofuran-3-yl)phenyl]acrylate (7). Compound 8 (80.0 mg, 0.25 mmol) was allowed to react with ethyl acrylate under non-migration reaction conditions (procedure A) at $80^{\circ} \mathrm{C}$ for $1 \mathrm{~d}$. The reaction mixture was chromatographed using $7: 1$ hexanes/ethyl acetate to afford $54.8 \mathrm{mg}(75 \%)$ of the indicated compound as a clear oil: ${ }^{1} \mathrm{H}$ $\operatorname{NMR}\left(\mathrm{CDCl}_{3}\right) \delta 1.24(\mathrm{t}, J=7.0 \mathrm{~Hz}, 3 \mathrm{H}), 4.18(\mathrm{q}, J=7.2 \mathrm{~Hz}, 2 \mathrm{H}), 6.44(\mathrm{~d}, J=16.0 \mathrm{~Hz}, 1 \mathrm{H})$, 7.24-7.28 (m, 1H), 7.33-7.58 (m, 6H), $7.60(\mathrm{~s}, 1 \mathrm{H}), 7.76(\mathrm{~d}, J=7.6 \mathrm{~Hz}, 1 \mathrm{H}), 7.86(\mathrm{~d}, J=15.6$ 
$\mathrm{Hz}, 1 \mathrm{H}) ;{ }^{13} \mathrm{C} \mathrm{NMR}\left(\mathrm{CDCl}_{3}\right) \delta 14.3,60.5,111.8,119.8,119.8,120.5,123.2,124.9,127.1$, 127.6, 128.2, 130.1, 130.7, 132.2, 133.7, 143.1, 143.5, 155.3, 166.8; IR $\left(\mathrm{CH}_{2} \mathrm{Cl}_{2}\right) 3060,2979$, 1711, 1633, $1452 \mathrm{~cm}^{-1}$; HRMS $m / z, 292.1104$ (calcd for $\mathrm{C}_{19} \mathrm{H}_{16} \mathrm{O}_{3}, 292.1099$ ).

Ethyl $\boldsymbol{E}$-3-(1-methyl-3-phenylindol-2-yl)acrylate (10). Compound 9 (83.2 mg, 0.25

mmol) was allowed to react with ethyl acrylate under our migration reaction conditions (procedure C) for $1 \mathrm{~d}$. The reaction mixture was chromatographed using 3:1 hexanes/ethyl acetate to afford $71.2 \mathrm{mg}(94 \%)$ of the indicated compound $\mathbf{1 0}$ as a yellow oil: ${ }^{1} \mathrm{H}$ NMR $\left(\mathrm{CDCl}_{3}\right) \delta 1.29(\mathrm{t}, J=7.2 \mathrm{~Hz}, 3 \mathrm{H}), 3.90(\mathrm{~s}, 3 \mathrm{H}), 4.22(\mathrm{q}, J=7.2 \mathrm{~Hz}, 2 \mathrm{H}), 6.23(\mathrm{~d}, J=16.4$ $\mathrm{Hz}, 1 \mathrm{H}), 7.12-7.14(\mathrm{~m}, 1 \mathrm{H}), 7.33-7.39(\mathrm{~m}, 3 \mathrm{H}), 7.45-7.48(\mathrm{~m}, 4 \mathrm{H}), 7.62(\mathrm{~d}, J=8.0 \mathrm{~Hz}, 1 \mathrm{H})$, $7.84(\mathrm{~d}, J=16.4 \mathrm{~Hz}, 1 \mathrm{H}) ;{ }^{13} \mathrm{C} \mathrm{NMR}\left(\mathrm{CDCl}_{3}\right) \delta 14.4,31.7,60.6,109.7,118.7,120.6,120.6$, $122.7,124.6,127.0,127.2,128.8,130.4,131.1,133.3,134.2,139.1,167.2 ; \mathrm{IR}\left(\mathrm{CH}_{2} \mathrm{Cl}_{2}\right)$ $3057,2975,2927,1708,1625,1369,1289,1180 \mathrm{~cm}^{-1}$; HRMS m/z 305.1420 (calcd for $\left.\mathrm{C}_{20} \mathrm{H}_{19} \mathrm{NO}_{2}, 305.1416\right)$.

Ethyl E-3-(2-(1-methylindol-3-yl)phenyl)acrylate (11). Compound $12(83.2 \mathrm{mg}$, $0.25 \mathrm{mmol}$ ) was allowed to react with ethyl acrylate under non-migration reaction conditions (procedure A) at $75^{\circ} \mathrm{C}$ for $2 \mathrm{~d}$. The reaction mixture was chromatographed using 3:1 hexanes/ethyl acetate to afford $64.4 \mathrm{mg}(85 \%)$ of the indicated compound as a white solid: mp 130-131 ${ }^{\circ} \mathrm{C} ;{ }^{1} \mathrm{H}$ NMR $\left(\mathrm{CDCl}_{3}\right) \delta 1.26(\mathrm{t}, J=7.2 \mathrm{~Hz}, 3 \mathrm{H}), 3.85(\mathrm{~s}, 3 \mathrm{H}), 4.19(\mathrm{q}, J=7.2$ Hz, 2H), $6.43(\mathrm{~d}, J=16.0 \mathrm{~Hz}, 1 \mathrm{H}), 7.01(\mathrm{~s}, 1 \mathrm{H}), 7.14-7.18(\mathrm{~m}, 1 \mathrm{H}), 7.27-7.39(\mathrm{~m}, 3 \mathrm{H}), 7.42-$ $7.46(\mathrm{~m}, 1 \mathrm{H}), 7.60-7.64(\mathrm{~m}, 2 \mathrm{H}), 7.73(\mathrm{~d}, J=8.0 \mathrm{~Hz}, 1 \mathrm{H}), 7.97(\mathrm{~d}, J=16.0 \mathrm{~Hz}, 1 \mathrm{H}) ;{ }^{13} \mathrm{C}$ $\operatorname{NMR}\left(\mathrm{CDCl}_{3}\right) \delta 14.4,33.0,60.3,109.5,113.9,118.5,120.0,120.1,122.2,126.6,127.0$, 127.4, 129.4, 129.9, 131.0, 133.2, 136.0, 137.0, 144.6, 167.2; IR $\left(\mathrm{CH}_{2} \mathrm{Cl}_{2}\right) 2918,2848,1712$, 
1631, $1314 \mathrm{~cm}^{-1}$; HRMS m/z 305.1420 (calcd for $\mathrm{C}_{20} \mathrm{H}_{19} \mathrm{NO}_{2}, 305.1416$ ); Anal. C, 78.36; H, 6.28; N, 4.45 (calcd for $\mathrm{C}_{20} \mathrm{H}_{19} \mathrm{NO}_{2}$ : C, 78.66; $\mathrm{H}, 6.27 ; \mathrm{N}, 4.59$ ).

Ethyl E-3-(3',5'-dimethylbiphen-2-yl)acrylate (14). Compound 13 (77.0 mg, 0.25 mmol) was allowed to react with ethyl acrylate under non-migration reaction conditions (procedure A) for $1 \mathrm{~d}$. The reaction mixture was chromatographed using 7:1 hexanes/ethyl acetate to afford $69.0 \mathrm{mg}(97 \%)$ of the indicated compound $\mathbf{1 4}$ as a clear oil: ${ }^{1} \mathrm{H} \mathrm{NMR}$ $\left(\mathrm{CDCl}_{3}\right) \delta 1.28(\mathrm{t}, J=7.2 \mathrm{~Hz}, 3 \mathrm{H}), 2.36(\mathrm{~s}, 6 \mathrm{H}), 4.20(\mathrm{q}, J=7.2 \mathrm{~Hz}, 2 \mathrm{H}), 6.38(\mathrm{~d}, J=16.0$ Hz, 1H), 6.92 (s, 2H), $7.02(\mathrm{~s}, 1 \mathrm{H}), 7.33-7.41(\mathrm{~m}, 3 \mathrm{H}), 7.67-7.69$ (m, 1H), 7.75 (d, J = 15.6 $\mathrm{Hz}, 1 \mathrm{H}) ;{ }^{13} \mathrm{C} \mathrm{NMR}\left(\mathrm{CDCl}_{3}\right) \delta 14.4,21.4,60.3,118.9,126.6,127.5,127.8,129.2,129.8$, 130.5, 132.6, 137.8, 139.9, 143.3, 143.9, 167.0; IR $\left(\mathrm{CH}_{2} \mathrm{Cl}_{2}\right) 2918,2976,1712,1632,1313$ $\mathrm{cm}^{-1}$; HRMS m/z 280.1468 (calcd for $\mathrm{C}_{19} \mathrm{H}_{20} \mathrm{O}_{2}, 280.1463$ ).

Ethyl $\boldsymbol{E}$-3-(2'-methylbiphen-2-yl)acrylate (17). Compound 16 (73.5 mg, 0.25 mmol) was allowed to react with ethyl acrylate under non-migration reaction conditions (procedure A) for $1 \mathrm{~d}$. The reaction mixture was chromatographed using 7:1 hexanes/ethyl acetate to afford $61.2 \mathrm{mg}(92 \%)$ of the indicated compound $\mathbf{1 7}$ as a yellow oil: ${ }^{1} \mathrm{H}$ NMR $\left(\mathrm{CDCl}_{3}\right) \delta 1.24(\mathrm{t}, J=7.2 \mathrm{~Hz}, 3 \mathrm{H}), 2.03(\mathrm{~s}, 3 \mathrm{H}), 4.16(\mathrm{q}, J=7.2 \mathrm{~Hz}, 2 \mathrm{H}), 6.33(\mathrm{~d}, J=16.0$ $\mathrm{Hz}, 1 \mathrm{H}), 7.10(\mathrm{~d}, J=7.2 \mathrm{~Hz}, 1 \mathrm{H}), 7.20-7.29(\mathrm{~m}, 4 \mathrm{H}), 7.37-7.44(\mathrm{~m}, 3 \mathrm{H}), 7.70-7.72(\mathrm{~m}, 1 \mathrm{H})$;

${ }^{13} \mathrm{C} \mathrm{NMR}\left(\mathrm{CDCl}_{3}\right) \delta 14.3,20.1,60.4,119.0,125.7,126.2,127.7,128.0,129.8,129.9,130.1$, 130.5, 133.0, 136.1, 139.6, 142.9, 143.0, 166.9; IR $\left(\mathrm{CH}_{2} \mathrm{Cl}_{2}\right) 3061,2978,2920,1713,1633$, $1177 \mathrm{~cm}^{-1}$; HRMS m/z 266.1312 (calcd for $\mathrm{C}_{18} \mathrm{H}_{18} \mathrm{O}_{2}, 266.1307$ ).

Ethyl $E$-3-(2'-methylbiphen-2-yl)acrylate (17) and ethyl $E$-3-(6-methylbiphen-2yl)acrylate (18). Compound $\mathbf{1 6}(73.5 \mathrm{mg}, 0.25 \mathrm{mmol})$ was allowed to react with ethyl acrylate under our migration reaction conditions (procedure $\mathrm{C}$ ) for $1 \mathrm{~d}$. The reaction mixture 
was chromatographed using 7:1 hexanes/ethyl acetate to afford $60.5 \mathrm{mg}(91 \%)$ of the indicated compounds as a yellow oil in a 65:35 ratio. Minor isomer 18: ${ }^{1} \mathrm{H} \mathrm{NMR}\left(\mathrm{CDCl}_{3}\right) \delta$ $2.08(\mathrm{~s}, 3 \mathrm{H}), 6.27(\mathrm{~d}, J=16.0 \mathrm{~Hz}, 1 \mathrm{H}), 7.54-7.56(\mathrm{~m}, 1 \mathrm{H})$ as characteristic peaks; ${ }^{13} \mathrm{C}$ NMR $\left(\mathrm{CDCl}_{3}\right) \delta 20.9,60.3,118.8,123.8,127.4,127.5,128.5,129.7,131.5,133.4,137.0,138.9$, 142.8, 143.9, 143.9 as characteristic peaks; HRMS $m / z, 266.1312$ (calcd for $\mathrm{C}_{18} \mathrm{H}_{18} \mathrm{O}_{2}$, 266.1307).

Ethyl $\boldsymbol{E}$-3-(3-methoxybiphen-2-yl)acrylate (20). Compound 19 (77.5 mg, 0.25 mmol) was allowed to react with ethyl acrylate under non-migration reaction conditions (procedure A) for $2 \mathrm{~d}$. The reaction mixture was chromatographed using 7:1 hexanes/ethyl acetate to afford $55.1 \mathrm{mg}(78 \%)$ of the indicated compound $\mathbf{2 0}$ as a clear oil: ${ }^{1} \mathrm{H}$ NMR $\left(\mathrm{CDCl}_{3}\right) \delta 1.25(\mathrm{t}, J=7.2 \mathrm{~Hz}, 3 \mathrm{H}), 3.94(\mathrm{~s}, 3 \mathrm{H}), 4.16(\mathrm{q}, J=7.2 \mathrm{~Hz}, 2 \mathrm{H}), 6.61(\mathrm{~d}, J=16.4$ $\mathrm{Hz}, 1 \mathrm{H}), 6.94-6.96(\mathrm{~m}, 2 \mathrm{H}), 7.28-7.41(\mathrm{~m}, 6 \mathrm{H}), 7.63(\mathrm{~d}, J=16.4 \mathrm{~Hz}, 1 \mathrm{H}) ;{ }^{13} \mathrm{C} \mathrm{NMR}\left(\mathrm{CDCl}_{3}\right)$ $\delta 14.3,55.7,60.2,110.0,121.5,122.4,123.0,127.5,128.2,129.9,130.0,139.8,140.6,145.5$, 159.3, 168.0; IR $\left(\mathrm{CH}_{2} \mathrm{Cl}_{2}\right)$ 3054, 2985, 2926, 1705, 1265, $\mathrm{cm}^{-1} ; \mathrm{HRMS} 282.1256 \mathrm{~m} / \mathrm{z}$ (calcd for $\left.\mathrm{C}_{18} \mathrm{H}_{18} \mathrm{O}_{3}, 282.1256\right)$.

Ethyl E-3-(3'-methoxybiphen-2-yl)acrylate (21). Compound 26 (77.5 mg, 0.25 mmol) was allowed to react with ethyl acrylate under non-migration reaction conditions (procedure A) for $2 \mathrm{~d}$. The reaction mixture was chromatographed using 7:1 hexanes/ethyl acetate to afford $60.0 \mathrm{mg}(85 \%)$ of the indicated compound as a clear oil: ${ }^{1} \mathrm{H} \mathrm{NMR}\left(\mathrm{CDCl}_{3}\right)$ $\delta 1.28(\mathrm{t}, J=7.2 \mathrm{~Hz}, 3 \mathrm{H}), 3.83(\mathrm{~s}, 3 \mathrm{H}), 4.21(\mathrm{q}, J=7.2 \mathrm{~Hz}, 2 \mathrm{H}), 6.39(\mathrm{~d}, J=16.0 \mathrm{~Hz}, 1 \mathrm{H})$, 6.85-6.95 (m, 3H), 7.32-7.43 (m, 4H), 7.68-7.70 (m, 1H), $7.75(\mathrm{~d}, J=16.0 \mathrm{~Hz}, 1 \mathrm{H}) ;{ }^{13} \mathrm{C}$ $\operatorname{NMR}\left(\mathrm{CDCl}_{3}\right) \delta 14.3,55.4,60.4,113.4,115.4,119.2,122.4,126.8,127.8,129.3,129.8$ 
130.4, 132.7, 141.3, 142.8, 143.8, 159.4, 166.9; $\mathrm{IR}\left(\mathrm{CH}_{2} \mathrm{Cl}_{2}\right) 3061,2980,1711,1632,1314$, 1177, $\mathrm{cm}^{-1}$; HRMS $\mathrm{m} / z$ (calcd for $\mathrm{C}_{18} \mathrm{H}_{18} \mathrm{O}_{3}, 282.1256$ ).

Ethyl E-3-(5-methoxybiphen-2-yl)acrylate (22). An authentic sample of compound 22 was prepared in two steps from 2-iodo-4-methoxybenzaldehyde ${ }^{8}\left[\mathrm{mp} 113-114{ }^{\circ} \mathrm{C} ;{ }^{1} \mathrm{H}\right.$ $\operatorname{NMR}\left(\mathrm{CDCl}_{3}\right) \delta 3.88(\mathrm{~s}, 3 \mathrm{H}), 6.99(\mathrm{dd}, J=8.8,2.4 \mathrm{~Hz}, 1 \mathrm{H}), 7.44(\mathrm{~d}, J=2.0 \mathrm{~Hz}, 1 \mathrm{H}), 7.85$ $(\mathrm{d}, J=8.8 \mathrm{~Hz}, 1 \mathrm{H}), 9.93(\mathrm{~s}, 1 \mathrm{H}) ;{ }^{13} \mathrm{C} \mathrm{NMR}\left(\mathrm{CDCl}_{3}\right) \delta 55.9,102.4,114.9,125.4,128.6,131.6$, 164.4, 194.5] in the following manner. 2-Iodo-4-methoxybenzaldehyde $(0.262 \mathrm{~g}, 1.0 \mathrm{mmol})$, $\mathrm{Pd}(\mathrm{OAc})_{2}(11.2 \mathrm{mg}, 0.05 \mathrm{mmol}), \mathrm{PPh}_{3}(26.2 \mathrm{mg}, 0.1 \mathrm{mmol}), \mathrm{CsF}(0.334 \mathrm{~g}, 2.2 \mathrm{mmol})$ and phenylboronic acid $(0.146 \mathrm{~g}, 1.2 \mathrm{mmol})$ in $\mathrm{DME}(5 \mathrm{ml})$ under $\mathrm{Ar}$ at $90^{\circ} \mathrm{C}$ were stirred for 3 h. The reaction mixture was then cooled to room temperature, diluted with diethyl ether (35 $\mathrm{mL})$ and washed with brine $(30 \mathrm{~mL})$. The aqueous layer was reextracted with diethyl ether $(15 \mathrm{~mL})$. The organic layers were combined, dried $\left(\mathrm{MgSO}_{4}\right)$, filtered, and the solvent removed under reduced pressure. The residue was purified by column chromatography on a silica gel column using 7:1 hexanes/ethyl acetate to afford $21.0 \mathrm{mg}$ (99\%) of 2-phenyl-4methoxybenzaldehyde as a clear oil: ${ }^{1} \mathrm{H}$ NMR $\left(\mathrm{CDCl}_{3}\right) \delta 3.91(\mathrm{~s}, 3 \mathrm{H}), 6.88(\mathrm{~d}, J=2.4 \mathrm{~Hz}$, 1H), 6.99-7.02 (m, 1H), 7.38-7.47 (m, 5H), $8.03(\mathrm{~d}, J=8.8 \mathrm{~Hz}, 1 \mathrm{H}), 9.84(\mathrm{~d}, J=0.4 \mathrm{~Hz}, 1 \mathrm{H})$; ${ }^{13} \mathrm{C}$ NMR $\left(\mathrm{CDCl}_{3}\right) \delta 55.7,114.0,115.2,127.4,128.3,128.4,129.9,130.0,137.9,148.6$, 163.6, 191.1. 2-Phenyl-4-methoxybenzaldehyde $(53.0 \mathrm{mg}, 0.25 \mathrm{mmol})$ and (carboethoxymethylene)triphenylphosphorane $(0.1305 \mathrm{~g}, 0.375 \mathrm{mmol})$ in $\mathrm{CH}_{2} \mathrm{Cl}_{2}(5 \mathrm{ml})$ were stirred at $60{ }^{\circ} \mathrm{C}$ under argon for $15 \mathrm{~h}$. The reaction mixture was allowed to cool to room temperature and the solvent evaporated under reduced pressure. The resulting yellow oil was purified by column chromatography on a silica gel column using 1:9 ethyl acetate/hexanes to afford $43.7 \mathrm{mg}(62 \%)$ of the desired compound 22 as a clear oil: ${ }^{1} \mathrm{H}$ NMR $\left(\mathrm{CDCl}_{3}\right) \delta 1.27(\mathrm{t}$, 
$J=7.2 \mathrm{~Hz}, 3 \mathrm{H}), 3.85(\mathrm{~s}, 3 \mathrm{H}), 4.18(\mathrm{q}, J=7.2 \mathrm{~Hz}, 2 \mathrm{H}), 6.29(\mathrm{~d}, J=15.9 \mathrm{~Hz}, 1 \mathrm{H}), 6.87(\mathrm{~d}, J=$ $2.7 \mathrm{~Hz}, 1 \mathrm{H}), 6.92-6.95(\mathrm{~m}, 1 \mathrm{H}), 7.29-7.33(\mathrm{~m}, 2 \mathrm{H}), 7.39-7.44(\mathrm{~m}, 3 \mathrm{H}), 7.64-7.69(\mathrm{~m}, 2 \mathrm{H})$;

${ }^{13} \mathrm{C} \mathrm{NMR}\left(\mathrm{CDCl}_{3}\right) \delta 14.5,55.7,60.4,114.2,115.4,117.0,125.6,127.9,128.5,128.5,129.9$, 140.1, 143.4, 145.1, 160.9, 167.4; IR $\left(\mathrm{CH}_{2} \mathrm{Cl}_{2}\right)$ 3056, 2978, 2937, 1709, 1630. 1600, 1484

$\mathrm{cm}^{-1}$; HRMS $\mathrm{m} / z$ (calcd for $\mathrm{C}_{18} \mathrm{H}_{18} \mathrm{O}_{3}, 282.1256$ ).

Ethyl E-3-(3-methoxybiphen-2-yl)acrylate (20), ethyl E-3-(3'-methoxybiphen-2yl)acrylate (21), and ethyl $E$-3-(5-methoxybiphen-2-yl)acrylate (22). Compound 19 (77.5 $\mathrm{mg}, 0.25 \mathrm{mmol}$ ) was allowed to react with ethyl acrylate under our migration reaction conditions (procedure $\mathrm{C}$ ) for $2 \mathrm{~d}$. The reaction mixture was chromatographed using 7:1 hexanes/ethyl acetate to afford $68.5 \mathrm{mg}(97 \%)$ of the indicated compounds as a clear oil in a 53:38:9 ratio. Similarly, compound $26(77.5 \mathrm{mg}, 0.25 \mathrm{mmol})$ was allowed to react with ethyl acrylate under our standard migration reaction conditions (Procedure C) for $1 \mathrm{~d}$. The reaction mixture was chromatographed using 7:1 hexanes/ethyl acetate to afford $61.3 \mathrm{mg}$ $(87 \%)$ of the indicated compounds as a clear oil in a 25:62:13 ratio.

\section{Suzuki reactions}

\section{General Procedure for the Suzuki Coupling of o-Iodobiaryls with Arylboronic} Acids under the Conditions Described by Wright et al ${ }^{9}$ (Suzuki Non-migration

Conditions): To a 2-dram vial was added the $o$-iodobiaryl $(0.25 \mathrm{mmol})$, the arylboronic acid (0.30 mmol, 1.2 equiv), $\mathrm{Pd}(\mathrm{OAc})_{2}(2.8 \mathrm{mg}, 5 \mathrm{~mol} \%), \mathrm{PPh}_{3}(6.5 \mathrm{mg}, 10 \mathrm{~mol} \%), \mathrm{CsF}(84.0$ mg, 2.2 equiv) and DME $(1.0 \mathrm{~mL})$. The mixture was flushed with $\mathrm{Ar}$ and stirred at $90{ }^{\circ} \mathrm{C}$ in an oil bath. The completion of the reaction was determined by the observation of palladium 
black. The reaction mixture was cooled and purified directly by column chromatography on a silica gel column.

\section{General Procedure for the Suzuki Coupling of $o$-Iodobiaryls with Arylboronic} Acids under Migration Conditions (Suzuki Migration Conditions): To a 2-dram vial was added the $o$-iodobiaryl $(0.25 \mathrm{mmol})$, the arylboronic acid $\left(0.35 \mathrm{mmol}, 1.4\right.$ equiv), $\mathrm{Pd}(\mathrm{OAc})_{2}$ (2.8 mg, $5 \mathrm{~mol} \%),\left(\mathrm{Ph}_{2} \mathrm{P}\right)_{2} \mathrm{CH}_{2}(\mathrm{dppm}, 4.8 \mathrm{mg}, 5 \mathrm{~mol} \%), \mathrm{CsO}_{2} \mathrm{CCMe}_{3}(\mathrm{CsPiv}, 117 \mathrm{mg}, 2.0$ equiv), $\mathrm{Me}_{3} \mathrm{CCO}_{2} \mathrm{H}$ (PA, $51 \mathrm{mg}, 2$ equiv), $\mathrm{H}_{2} \mathrm{O}$ (90 mg, 20 equiv) and DMF (4.0 mL). The mixture was flushed with $\mathrm{Ar}$ and stirred at $100{ }^{\circ} \mathrm{C}$ in an oil bath. The completion of the reaction was determined by the observation of palladium black. The reaction mixture was cooled, diluted with ether and washed with satd aq $\mathrm{NaHCO}_{3}$. The organic layer was dried $\left(\mathrm{Na}_{2} \mathrm{SO}_{4}\right)$, filtered and evaporated. The residue was purified by column chromatography on a silica gel column and the molar ratio of the products was determined by ${ }^{1} \mathrm{H}$ NMR spectroscopic analysis.

Methyl 4"-methyl-o-terphenyl-4-carboxylate (28a). This compound was prepared employing 1a and 27a under Suzuki non-migration conditions for $8 \mathrm{~h}$. The reaction mixture was chromatographed using 9:1 hexanes/EtOAc to afford a 62\% yield of the indicated compound as a colorless oil: ${ }^{1} \mathrm{H}$ NMR $\left(\mathrm{CDCl}_{3}\right) \delta 2.30(\mathrm{~s}, 3 \mathrm{H}), 3.89(\mathrm{~s}, 3 \mathrm{H})$, 6.97-7.04 (m, 4H), 7.20-7.23 (m, 2H), 7.40-7.44 (m, 4H), 7.87-7.90 (m, 2H); ${ }^{13} \mathrm{C} \mathrm{NMR}\left(\mathrm{CDCl}_{3}\right) \delta$ 21.3, 52.3, 127.6, 128.3 (2), 129.0, 129.4, 129.9, 130.1, 130.6, 131.0, 136.6, 138.3, 139.7, 140.9, 146.9, 167.4; IR (neat, $\mathrm{cm}^{-1}$ ) 3023, 2918, 1724; HRMS calcd for $\mathrm{C}_{21} \mathrm{H}_{18} \mathrm{O}_{2}: 302.1307$. Found: 302.1313.

4"-Methyl-o-terphenyl. This compound was prepared employing $\mathbf{1 a}$ and $\mathbf{2 7 b}$ under Suzuki non-migration conditions for $40 \mathrm{~h}$. The reaction mixture was chromatographed using 
50:1 hexanes/EtOAc to afford an $85 \%$ yield of the indicated compound as a colorless oil, which crystallizes upon cooling and standing: $\mathrm{mp} 74-75^{\circ} \mathrm{C}\left(\right.$ lit. $\left.{ }^{10} \mathrm{mp} 74-75^{\circ} \mathrm{C}\right) ;{ }^{13} \mathrm{C} \mathrm{NMR}$ $\left(\mathrm{CDCl}_{3}\right) \delta 21.4,126.7,127.5,127.7,128.1,128.9,130.0,130.2,130.9,136.3,138.8,140.78$ 140.79, 142.0 (one $\mathrm{sp}^{2}$ carbon missing due to overlap); all other spectral properties are identical to those previously reported..$^{10,11}$

4-Methoxy-4"'-methyl-o-terphenyl. This compound was prepared employing 1a and 27c under Suzuki non-migration conditions for $18 \mathrm{~h}$. The reaction mixture was chromatographed using 40:1 hexanes/EtOAc to afford a 93\% yield of the indicated compound as a colorless oil: ${ }^{13} \mathrm{C} \mathrm{NMR}\left(\mathrm{CDCl}_{3}\right) \delta 21.2,55.2,113.4,127.2,127.4,128.8$, $129.8,130.6,130.7,131.0,134.2,136.0,138.8,140.2,140.5,158.3$; all other spectral properties are identical to those previously reported. ${ }^{12}$

Methyl 4"'-methoxy-o-terphenyl-4-carboxylate (28b). This compound was prepared employing $\mathbf{1 b}$ and 27a under Suzuki non-migration conditions for $18 \mathrm{~h}$. The reaction mixture was chromatographed using 6:1 hexanes/EtOAc to afford a $92 \%$ yield of the indicated compound as a colorless oil, which crystallizes upon cooling and standing: mp 113-114 ${ }^{\circ} \mathrm{C} ;{ }^{1} \mathrm{H} \mathrm{NMR}\left(\mathrm{CDCl}_{3}\right) \delta 3.76(\mathrm{~s}, 3 \mathrm{H}), 3.89(\mathrm{~s}, 3 \mathrm{H}), 6.74(\mathrm{~d}, J=8.8 \mathrm{~Hz}, 2 \mathrm{H}), 7.02(\mathrm{~d}$, $J=8.8 \mathrm{~Hz}, 2 \mathrm{H}), 7.22(\mathrm{~d}, J=8.4 \mathrm{~Hz}, 2 \mathrm{H}), 7.41(\mathrm{~m}, 4 \mathrm{H}), 7.90(\mathrm{~d}, J=8.4 \mathrm{~Hz}, 2 \mathrm{H}) ;{ }^{13} \mathrm{C} \mathrm{NMR}$ $\left(\mathrm{CDCl}_{3}\right) \delta 52.1,55.2,113.6,127.3,128.1,128.2,129.3,129.9,130.5,130.7,131.0,133.4$, 139.4, 140.3, 146.7, 158.5, 167.2; IR (neat, $\mathrm{cm}^{-1}$ ) 3024, 2951, 1721; HRMS calcd for $\mathrm{C}_{21} \mathrm{H}_{18} \mathrm{O}_{3}$ : 318.1256. Found: 318.1261.

Ethyl methyl o-terphenyl-4,4"'-dicarboxylate (28c). This compound was prepared employing 1c and 27a under Suzuki non-migration conditions for $16 \mathrm{~h}$. The reaction mixture was chromatographed using 6:1 hexanes/EtOAc to afford a $98 \%$ yield of the indicated 
compound as a colorless oil, which crystallizes upon cooling and standing: $\mathrm{mp} 103-104{ }^{\circ} \mathrm{C}$; ${ }^{1} \mathrm{H}$ NMR $\left(\mathrm{CDCl}_{3}\right) \delta 1.37$ (t, $\left.J=7.2 \mathrm{~Hz}, 3 \mathrm{H}\right), 3.89$ (s, 3H), 4.35 (q, $\left.J=7.2 \mathrm{~Hz}, 2 \mathrm{H}\right), 7.18$ (dd, $J=2.4,8.4 \mathrm{~Hz}, 4 \mathrm{H}), 7.45(\mathrm{~m}, 4 \mathrm{H}), 7.89(\mathrm{dd}, J=2.0,8.4 \mathrm{~Hz}, 4 \mathrm{H}) ;{ }^{13} \mathrm{C} \mathrm{NMR}\left(\mathrm{CDCl}_{3}\right) \delta 14.4$, $52.1,61.0,128.28,128.30,128.5,128.8,129.37,129.40,129.87,129.92,130.58,130.60$, 139.6, 139.7, 145.8, 145.9, 166.5, 167.0; IR (neat, $\mathrm{cm}^{-1}$ ) 3024, 2985, 1720; HRMS calcd for $\mathrm{C}_{23} \mathrm{H}_{20} \mathrm{O}_{4}: 360.1362$. Found: 360.1371.

Methyl 4"'-nitro-o-terphenyl-4-carboxylate (28d). This compound was prepared employing 1d and 27a under Suzuki non-migration conditions for $5 \mathrm{~h}$. The reaction mixture was chromatographed using 4:1 hexanes/EtOAc to afford a 95\% yield of the indicated compound as a colorless solid: $\mathrm{mp} 149-150{ }^{\circ} \mathrm{C} ;{ }^{1} \mathrm{H} \mathrm{NMR}\left(\mathrm{CDCl}_{3}\right) \delta 3.90(\mathrm{~s}, 3 \mathrm{H}), 7.18(\mathrm{~d}, J=$ $8.4 \mathrm{~Hz}, 2 \mathrm{H}), 7.27(\mathrm{~d}, J=9.0 \mathrm{~Hz}, 2 \mathrm{H}), 7.45-7.51(\mathrm{~m}, 4 \mathrm{H}), 7.91(\mathrm{~d}, J=8.4 \mathrm{~Hz}, 2 \mathrm{H}), 8.07$ (d, $J$ $=8.7 \mathrm{~Hz}, 2 \mathrm{H}) ;{ }^{13} \mathrm{C} \mathrm{NMR}\left(\mathrm{CDCl}_{3}\right) \delta 52.4,123.6,128.7,129.0,129.1,129.8,130.1,130.6$, 130.8, 130.9, 138.5, 139.9, 145.6, 146.8, 148.2, 167.0; IR (neat, $\mathrm{cm}^{-1}$ ) 3011, 2949, 1722, 1599; HRMS calcd for $\mathrm{C}_{20} \mathrm{H}_{15} \mathrm{NO}_{4}$ : 333.1001. Found: 333.1007 .

2-(4-Methoxycarbonylphenyl)-3-phenylbenzofuran (30). This compound was prepared employing 5 and 27a under our Suzuki migration conditions for $3 \mathrm{~h}$. The reaction mixture was chromatographed using 9:1 hexanes/EtOAc to afford a 79\% yield of the indicated compound as a white solid: $\mathrm{mp} 122-123{ }^{\circ} \mathrm{C} ;{ }^{1} \mathrm{H}$ NMR $\left(\mathrm{CDCl}_{3}\right) \delta 3.90(\mathrm{~s}, 3 \mathrm{H}), 7.23-$ $7.27(\mathrm{~m}, 1 \mathrm{H})$, 7.34-7.38 (m, H), 7.43-7.51 (m, 6H), 7.56-7.58 (m, 1H), 7.71-7.73 (m, 2H), 7.96-7.98 (m, 2H); ${ }^{13} \mathrm{C} \mathrm{NMR}\left(\mathrm{CDCl}_{3}\right) \delta 52.2,111.3,119.6,120.4,123.2,125.5,126.6,128.1$, $129.2,129.4,129.7,130.1,132.4,134.9,149.3,154.2,166.7$ (one $\mathrm{sp}^{2}$ carbon missing due to overlap); IR (neat, $\mathrm{cm}^{-1}$ ) 2917, 2849, 1727; HRMS calcd for $\mathrm{C}_{22} \mathrm{H}_{16} \mathrm{O}_{3}$ : 328.1099. Found: 328.1104. Anal. calcd for $\mathrm{C}_{22} \mathrm{H}_{16} \mathrm{O}_{3}:$ C, 80.47; H, 4.91. Found: C, 80.12; H, 4.81. 
2-(4-Methoxycarbonylphenyl)-1-methyl-3-phenylindole (32). This compound was prepared employing 9 and 27a under our Suzuki migration conditions for $3 \mathrm{~h}$. The reaction mixture was chromatographed using 6:1 hexanes/EtOAc to afford a 67\% yield of the indicated compound as a white solid: $\mathrm{mp} 122-123{ }^{\circ} \mathrm{C} ;{ }^{1} \mathrm{H} \mathrm{NMR}\left(\mathrm{CDCl}_{3}\right) \delta 3.71(\mathrm{~s}, 3 \mathrm{H}), 3.94$ (s, 3H), $7.19(\mathrm{~m}, 2 \mathrm{H}), 7.27(\mathrm{~m}, 4 \mathrm{H}), 7.32(\mathrm{t}, J=7.2 \mathrm{~Hz}, 1 \mathrm{H}), 7.71(\mathrm{~m}, 3 \mathrm{H}), 7.78(\mathrm{~d}, J=8.0$ $\mathrm{Hz}, 1 \mathrm{H}), 8.04(\mathrm{~d}, J=8.0 \mathrm{~Hz}, 2 \mathrm{H}) ;{ }^{13} \mathrm{C} \mathrm{NMR}\left(\mathrm{CDCl}_{3}\right) \delta 31.2,52.3,109.8,116.2,119.9,120.5$, $122.8,125.9,127.0,128.4,129.5,129.7,130.0,131.2,134.8,136.5,136.7,137.8,166.9 ;$ IR (neat, $\mathrm{cm}^{-1}$ ) 3053, 2950, 1723; HRMS calcd for $\mathrm{C}_{23} \mathrm{H}_{19} \mathrm{NO}_{2}$ : 341.1416. Found: 341.1421 . Methyl 5'-methyl-o-terphenyl-4-carboxylate (29a). This compound was prepared employing 4a and 27a under Suzuki non-migration conditions for $12 \mathrm{~h}$. The reaction mixture was chromatographed using 10:1 hexanes/EtOAc to afford a 90\% yield of the indicated compound as a colorless oil: ${ }^{1} \mathrm{H}$ NMR $\left(\mathrm{CDCl}_{3}\right) \delta 2.42(\mathrm{~s}, 3 \mathrm{H}), 3.86(\mathrm{~s}, 3 \mathrm{H}), 7.07(\mathrm{~m}, 2 \mathrm{H})$, 7.14-7.26 (m, 7H), 7.32 (m, 1H), $7.86(\mathrm{dt}, J=8.4,1.8 \mathrm{~Hz}, 2 \mathrm{H}) ;{ }^{13} \mathrm{C} \mathrm{NMR}\left(\mathrm{CDCl}_{3}\right) \delta 21.3$, $52.3,126.8,128.26,128.34,129.1,129.4,130.1,130.2,130.9,131.4,137.6,138.1,139.6$, 141.2, 146.8, 167.3; IR (neat, $\mathrm{cm}^{-1}$ ) 3025, 2950, 1727; HRMS calcd for $\mathrm{C}_{21} \mathrm{H}_{18} \mathrm{O}_{2}: 302.1307$. Found: 302.1313.

5'-Methyl-o-terphenyl. This compound was prepared employing $\mathbf{4 a}$ and $\mathbf{2 7 b}$ under Suzuki non-migration conditions for $8 \mathrm{~h}$. The reaction mixture was chromatographed using 50:1 hexanes/EtOAc to afford a 93\% yield of the indicated compound as a colorless oil. All spectral properties are identical to those previously reported. ${ }^{13}$

4-Methoxy-5'-methyl-o-terphenyl. This compound was prepared employing $4 \mathbf{a}$ and 27c under Suzuki non-migration conditions for $16 \mathrm{~h}$. The reaction mixture was chromatographed using 30:1 hexanes/EtOAc to afford a 94\% yield of the indicated 
compound as a colorless oil, which crystallizes upon cooling and standing: $\mathrm{mp} 107-108^{\circ} \mathrm{C}$; ${ }^{1} \mathrm{H}$ NMR $\left(\mathrm{CDCl}_{3}\right) \delta 2.42(\mathrm{~s}, 3 \mathrm{H}), 3.75(\mathrm{~s}, 3 \mathrm{H}), 6.73(\mathrm{~m}, 2 \mathrm{H}), 7.05(\mathrm{~m}, 2 \mathrm{H}), 7.10-7.22(\mathrm{~m}, 7 \mathrm{H})$, $7.30(\mathrm{~d}, J=8.4 \mathrm{~Hz}, 1 \mathrm{H}) ;{ }^{13} \mathrm{C} \mathrm{NMR}\left(\mathrm{CDCl}_{3}\right) \delta 21.4,55.4,113.6,126.4,128.1,130.2,130.9$, 131.2, 131.6, 134.3, 137.4, 137.9, 140.2, 141.9, 158.5 (one $\mathrm{sp}^{2}$ carbon missing due to overlap); IR (neat, $\mathrm{cm}^{-1}$ ) 3026, 2931, 1516; HRMS calcd for $\mathrm{C}_{20} \mathrm{H}_{18} \mathrm{O}: 274.1358$. Found: 274.1362.

Methyl 5'-methoxy-o-terphenyl-4-carboxylate (29b). This compound was prepared employing $4 \mathbf{b}$ and 27a under Suzuki non-migration conditions for $16 \mathrm{~h}$. The reaction mixture was chromatographed using 6:1 hexanes/EtOAc to afford a 94\% yield of the indicated compound as a colorless oil, which crystallizes upon cooling and standing: mp 113-114 ${ }^{\circ} \mathrm{C} ;{ }^{1} \mathrm{H}$ NMR $\left(\mathrm{CDCl}_{3}\right) \delta 3.87$ (s, 3H), $3.88(\mathrm{~s}, 3 \mathrm{H}), 6.95-7.01(\mathrm{~m}, 2 \mathrm{H})$, 7.02-7.08 (m, 2H), 7.15-7.23 (m, 5H), 7.36 (d, $J=8.4 \mathrm{~Hz}, 1 \mathrm{H}), 7.88(\mathrm{~d}, J=8.4 \mathrm{~Hz}, 2 \mathrm{H}) ;{ }^{13} \mathrm{C} \mathrm{NMR}\left(\mathrm{CDCl}_{3}\right)$ $\delta 52.3,55.7,113.8,116.0,126.6,128.2,128.5,129.5,130.09,130.14,132.1,133.6,140.9$, 141.0, 146.6, 159.2, 167.3; IR (neat, $\mathrm{cm}^{-1}$ ) 3027, 2951, 1723; HRMS calcd for $\mathrm{C}_{21} \mathrm{H}_{18} \mathrm{O}_{3}$ : 318.1256. Found: 318.1263 .

Ethyl methyl $o$-terphenyl-5',4-dicarboxylate (29c). This compound was prepared employing $4 \mathbf{c}$ and 27a under Suzuki non-migration conditions for $16 \mathrm{~h}$. The reaction mixture was chromatographed using 5:1 hexanes/EtOAc to afford a 97\% yield of the indicated compound as a colorless oil, which crystallizes upon cooling and standing: $\mathrm{mp} 105-106{ }^{\circ} \mathrm{C}$; ${ }^{1} \mathrm{H} \mathrm{NMR}\left(\mathrm{CDCl}_{3}\right) \delta 1.41(\mathrm{t}, J=7.2 \mathrm{~Hz}, 3 \mathrm{H}), 3.89(\mathrm{~s}, 3 \mathrm{H}), 4.42(\mathrm{q}, J=7.2 \mathrm{~Hz}, 2 \mathrm{H}), 7.11(\mathrm{~m}$, 2H), 7.20-7.24 (m, 5H), $7.51(\mathrm{~d}, J=8.4 \mathrm{~Hz}, 1 \mathrm{H}), 7.90(\mathrm{~d}, J=8.4 \mathrm{~Hz}, 2 \mathrm{H}), 8.10(\mathrm{~m}, 2 \mathrm{H}) ;{ }^{13} \mathrm{C}$ NMR $\left(\mathrm{CDCl}_{3}\right) \delta 14.6,52.3,61.4,127.6,128.4,128.8,129.3,129.6,129.9,130.0,130.1$, 
131.1, 131.8, 139.9, 140.3, 145.3, 145.7, 166.5, 167.1; IR (neat, $\mathrm{cm}^{-1}$ ) 3027, 2983, 1723;

HRMS calcd for $\mathrm{C}_{23} \mathrm{H}_{20} \mathrm{O}_{4}$ : 360.1362 . Found: 360.1371 .

Methyl 5'-nitro-o-terphenyl-4-dicarboxylate (29d). This compound was prepared employing 4d and 27a under Suzuki non-migration conditions for $5 \mathrm{~h}$. The reaction mixture was chromatographed using 5:1 hexanes/EtOAc to afford an 84\% yield of the indicated compound as a colorless solid: $\mathrm{mp} 124-126{ }^{\circ} \mathrm{C} ;{ }^{1} \mathrm{H} \mathrm{NMR}\left(\mathrm{CDCl}_{3}\right) \delta 3.91(\mathrm{~s}, 3 \mathrm{H}), 7.10-7.13$ (m, 2H), 7.21-7.28 (m, 5H), $7.61(\mathrm{~d}, J=8.1 \mathrm{~Hz}, 1 \mathrm{H}), 7.93(\mathrm{dd}, J=6.6,1.6 \mathrm{~Hz}, 2 \mathrm{H}), 8.27-$ $8.31(\mathrm{~m}, 2 \mathrm{H}) ;{ }^{13} \mathrm{C} \mathrm{NMR}\left(\mathrm{CDCl}_{3}\right) \delta 52.4,123.1,125.6,128.3,128.7,129.5,129.7,129.8$, 130.0, 131.9, 139.1, 141.1, 144.2, 147.35, 147.41, 166.9; IR (neat, $\mathrm{cm}^{-1}$ ) 3063, 2951, 1723, 1611; HRMS calcd for $\mathrm{C}_{20} \mathrm{H}_{15} \mathrm{NO}_{4}$ : 333.1001. Found: 333.1007 .

Methyl 4-[2-(benzofuran-3-yl)phenyl]benzoate (31). This compound was prepared employing 8 and 27a under Suzuki non-migration conditions for $6 \mathrm{~h}$. The reaction mixture was chromatographed using 9:1 hexanes/EtOAc to afford a 75\% yield of the indicated compound as a colorless oil: ${ }^{1} \mathrm{H}$ NMR $\left(\mathrm{CDCl}_{3}\right) \delta 3.87(\mathrm{~s}, 3 \mathrm{H}), 7.11-7.14(\mathrm{~m}, 1 \mathrm{H}), 7.21-7.27$ $(\mathrm{m}, 2 \mathrm{H}), 7.30-7.34(\mathrm{~m}, 3 \mathrm{H}), 7.42-7.49(\mathrm{~m}, 4 \mathrm{H}), 7.58-7.60(\mathrm{~m}, 1 \mathrm{H}), 7.87-7.89(\mathrm{~m}, 2 \mathrm{H}) ;{ }^{13} \mathrm{C}$ $\operatorname{NMR}\left(\mathrm{CDCl}_{3}\right) \delta 52.3,111.7,120.6,121.2,123.0,124.6,127.4,128.3,128.4,128.8,129.5$, 129.6, 130.1, 130.8, 131.1, 140.7, 143.1, 146.4, 155.3, 167.2; IR (neat, $\mathrm{cm}^{-1}$ ) 2973, 2850, 1723; HRMS calcd for $\mathrm{C}_{22} \mathrm{H}_{16} \mathrm{O}_{3}$ : 328.1099. Found: 328.1104 .

Methyl 4-[2-(1-methylindol-3-yl)phenyl]benzoate (33). This compound was prepared employing 12 and 27a under Suzuki non-migration conditions for $16 \mathrm{~h}$. The reaction mixture was chromatographed using 6:1 hexanes/EtOAc to afford a 79\% yield of the indicated compound as a white solid: $\mathrm{mp} 147-148{ }^{\circ} \mathrm{C}$; ${ }^{1} \mathrm{H}$ NMR $\left(\mathrm{CDCl}_{3}\right) \delta 3.64(\mathrm{~s}, 3 \mathrm{H}), 3.88$ (s, 3H), $7.06(\mathrm{t}, J=7.2 \mathrm{~Hz}, 1 \mathrm{H}), 7.21(\mathrm{t}, J=7.2 \mathrm{~Hz}, 1 \mathrm{H}), 7.28(\mathrm{~d}, J=8.0 \mathrm{~Hz}, 1 \mathrm{H}), 7.31(\mathrm{~d}, J$ 
$=8.0 \mathrm{~Hz}, 2 \mathrm{H}), 7.40(\mathrm{~m}, 1 \mathrm{H}), 7.44(\mathrm{~m}, 2 \mathrm{H}), 7.54(\mathrm{~d}, J=8.0 \mathrm{~Hz}, 1 \mathrm{H}), 7.65(\mathrm{~d}, J=8.0 \mathrm{~Hz}, 1 \mathrm{H})$, $7.85(\mathrm{~d}, J=8.0 \mathrm{~Hz}, 2 \mathrm{H}) ;{ }^{13} \mathrm{C} \mathrm{NMR}\left(\mathrm{CDCl}_{3}\right) \delta 32.8,52.1,109.3,114.9,119.6,119.9,121.8$, 126.6, 127.2, 128.0, 128.6, 129.2, 129.6, 130.6, 131.3, 133.5, 136.8, 140.0, 147.4, 167.2; IR

(neat, $\mathrm{cm}^{-1}$ ) 3056, 2950, 1719; HRMS calcd for $\mathrm{C}_{23} \mathrm{H}_{19} \mathrm{NO}_{2}$ : 341.1416. Found: 341.1422 .

\section{Mass Spectral Data for Deuteration Experiments}

Ethyl $\boldsymbol{E}$-3-(biphen-2-yl)acrylate $\left(\mathrm{C}_{17} \mathrm{H}_{16} \mathrm{O}_{2}\right.$, nominal mass 252)

${ }^{1} \mathrm{H} \mathrm{NMR}\left(\mathrm{CDCl}_{3}\right) \delta 1.30(\mathrm{t}, J=7.1 \mathrm{~Hz}, 3 \mathrm{H}), 2.22(\mathrm{q}, J=7.2 \mathrm{~Hz}, 2 \mathrm{H}), 6.43(\mathrm{~d}, J=15.9 \mathrm{~Hz}$, 1H), 7.31-7.49 (m, 8H), 7.70-7.79 (m, 2H).

Mass spectral data near 252:

$\begin{array}{lrrr}\mathrm{m} / \mathrm{z} & 251 & 252 & 253 \\ \text { rel intensity } & 0 & 100 & 18\end{array}$

Deuterated ethyl $\boldsymbol{E}$-3-(biphen-2-yl)acrylate (after synthetic run in $\mathrm{D}_{2} \mathrm{O}$ )

${ }^{1} \mathrm{H} \mathrm{NMR}\left(\mathrm{CDCl}_{3}\right) \delta 1.32(\mathrm{t}, J=7.1 \mathrm{~Hz}, 3 \mathrm{H}), 2.24(\mathrm{q}, J=7.2 \mathrm{~Hz}, 2 \mathrm{H}), 6.45(\mathrm{~d}, J=15.9 \mathrm{~Hz}$, 1H), 7.35-7.49 (m, ca. 6H), 7.73-7.81 (m, 2H).

${ }^{2} \mathrm{H}$ NMR (acetone-d $\left.{ }_{6}\right) \delta 7.39$ (s)

MS data (FM 252)

$\mathrm{m} / \mathrm{z} \quad 251 \quad 252 \quad 253 \quad 254 \quad 255 \quad 256$

$\begin{array}{lllllll}\text { rel intensity } & 12 & 30 & 55 & 100 & 90 & 50\end{array}$ 
Mass spectral data for 3d (nominal mass 324)

$\mathrm{m} / \mathrm{z} \quad 323 \quad 324 \quad 325$

intensity $\quad 100 \quad 30 \quad 0$

MS data for 3d, prepared with $\mathrm{D}_{2} \mathrm{O}$

$\begin{array}{llllll}\mathrm{m} / \mathrm{z} & 323 & 324 & 325 & 326 & 327 \\ \text { rel intensity } & \text { a } & \text { a } & \sim 0 & 50 & 100\end{array}$

${ }^{a}$ Edge of noise threshold spanning large $m / z$ range, $c a .4$ relative to 327

\section{Computational Documentation}

\section{A simplified computational model, based on naked anions}

As noted in the main text, the data on the equilibrium position of palladation appear to correlate with the $\mathrm{C}-\mathrm{H}$ acidities of the potential positions. In order to predict this in advance, ideally, high quality calculations could be carried out on a whole series of Pd complexes with appropriate ligands to establish energy differences between the various isomers, instead of the small set we reported in the main text. However, we thought a simplified model, using the relative stabilities of the anions illustrated in Scheme S1, might still provide a qualitative tool if there was a physical basis underlying the apparent correlation. The numbering used in Scheme S1 and in the tables below is consistent with the main text. 


\section{Scheme S1.}

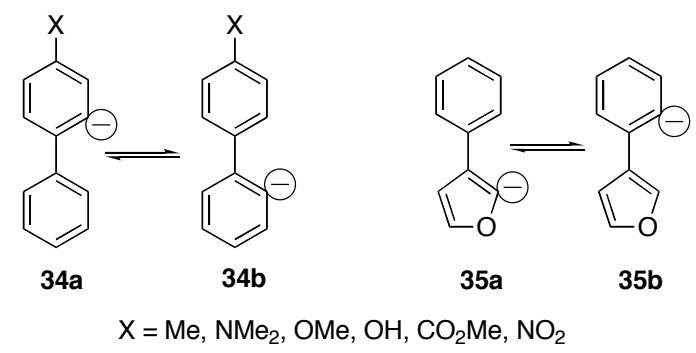

The energy differences, and, thus, hypothetical equilibrium distributions of the anions, were determined from $\Delta \mathrm{H}$ and $\Delta \mathrm{S}$ values as estimated from MP2/6$311+\mathrm{G}(\mathrm{d}, \mathrm{p}) / / \mathrm{HF} / 6-31+\mathrm{G}(\mathrm{d})$ and B3LYP/6-311+G(d,p)//HF/6-31+G(d) total electronic and vibrational calculations. Recognizing the shortcomings of the highly simplified modeling of the anions for the organometallic compounds and the lack of solvation, the originally determined $\Delta \mathrm{G}$ values were - quite empirically - multiplied by a factor such that the predicted ratio of ions and the product ratio for the Suzuki coupling products of the biphenyl bearing a $\mathrm{CO}_{2}$ Et group would match. It was assumed that solvation/coordination would not be greatly influenced by the substituent, and thus that this correction factor would be approximately constant for a series of related molecules. The adjusted values of $\Delta \mathrm{G}$ were used to determine the equilibrium constant, $\mathrm{K}$, and subsequently the ratios for the rest of the anions. The results are given in Tables $\mathrm{S} 1$ and S2, using MP2/6-311+G(d,p)//RHF/631+G(d,p) and B3LYP/6-311+G(d,p)//RHF/6-31+G(d,p) energies, respectively. 
Table S1. $\Delta \mathrm{H}, \Delta \mathrm{S}, \Delta \mathrm{G}, \mathrm{K}$, and Calculated Ratios for Substituted Biphenyl and Furan

Carbanions Using MP2 Energies.

\begin{tabular}{|c|c|c|c|c|c|c|c|c|}
\hline 34, $X=$ & $\Delta \mathrm{H}^{a}$ & $\mathrm{~T} \Delta \mathrm{S}^{b}$ & $\Delta \mathrm{G}^{b}$ & $\Delta \mathrm{G}_{\text {adjusted }}{ }^{c}$ & $\mathrm{~K}_{\text {adjusted }}{ }^{d}$ & Calc'd 34a:34b ${ }^{e}$ & $\begin{array}{l}\text { Expt, } \\
\text { Heck }^{\text {f }}\end{array}$ & $\begin{array}{c}\text { Expt, } \\
\text { Suzuki }^{g}\end{array}$ \\
\hline $\mathrm{Me}$ & 0.14 & -0.13 & 0.27 & 0.04 & 0.93 & $52: 48$ & $\sim 50: 50$ & $\sim 50: 50$ \\
\hline $\mathrm{NMe}_{2}$ & 1.04 & -0.09 & 1.13 & 0.17 & 0.75 & $57: 43$ & $\sim 48: 52$ & \\
\hline $\mathrm{OMe}$ & 1.61 & 0.10 & 1.51 & 0.23 & 0.68 & $59: 41$ & $\sim 50: 50$ & $\sim 60: 40$ \\
\hline $\mathrm{CO}_{2} \mathrm{Me}$ & 1.91 & -0.16 & 2.07 & 0.31 & 0.59 & $63: 37$ & $\sim 55: 45$ & $\sim 63: 37$ \\
\hline $\mathrm{OH}$ & 2.46 & 0.20 & 2.26 & 0.34 & 0.56 & $64: 36$ & & \\
\hline $\mathrm{NO}_{2}$ & 5.37 & -0.33 & 5.70 & 0.86 & 0.24 & $81: 19$ & $\sim 64: 36$ & $\sim 80: 20$ \\
\hline Furan $^{\mathrm{h}}$ & 11.61 & 0.14 & 11.47 & 1.72 & 0.05 & $95: 5$ & $\sim 97: 3$ & $\sim 97: 3$ \\
\hline $\begin{array}{l}{ }^{a} \text { Calcula } \\
=298.15 \\
\text { from diff } \\
\text { different } \\
\mathbf{6} \text { and } 7 .\end{array}$ & from th & MP2 en & gies for & forward & ion in & $\begin{array}{l}\text { ne } 10 \text { in units of } 1 \\
\text { om } \mathrm{K}_{\text {adjusted }}{ }^{f} \text { Ave }\end{array}$ & mol. ${ }^{b}$ In $\mathrm{k}$ & $\begin{array}{l}\text { 1/ } / \mathrm{mol}, \mathrm{T} \\
\text { io of } 3: 2 \\
\text { from } \\
\text { ct ratio of }\end{array}$ \\
\hline
\end{tabular}

Table S2. $\Delta \mathrm{H}, \Delta \mathrm{S}, \Delta \mathrm{G}, \mathrm{K}$, and Calculated Ratios for Substituted Biphenyl and Furan Carbanions Using B3LYP Energies.

\begin{tabular}{|c|c|c|c|c|c|c|c|c|}
\hline $34, X$ & $\Delta \mathrm{H}^{a}$ & $\mathrm{~T} \Delta \mathrm{S}^{b}$ & $\Delta \mathrm{G}^{b}$ & $\Delta \mathrm{G}_{\text {adjusted }}{ }^{c}$ & $\mathrm{~K}_{\text {adjusted }}{ }^{d}$ & Calc'd 34a:34b ${ }^{e}$ & $\begin{array}{l}\text { Expt, } \\
\text { Heck }^{f}\end{array}$ & $\begin{array}{c}\text { Expt, } \\
\text { Suzuki }^{8}\end{array}$ \\
\hline $\mathrm{Me}$ & 0.52 & -0.13 & 0.65 & 0.10 & 0.85 & $54: 46$ & $\sim 50: 50$ & $\sim 50: 50$ \\
\hline OMe & 2.07 & 0.10 & 1.97 & 0.30 & 0.61 & $62: 48$ & $\sim 50: 50$ & $\sim 60: 40$ \\
\hline $\mathrm{CO}_{2} \mathrm{Me}$ & 0.25 & -0.16 & 0.41 & 0.06 & 0.90 & $53: 47$ & $\sim 55: 45$ & $\sim 63: 37$ \\
\hline $\mathrm{OH}$ & 3.39 & 0.20 & 3.19 & 0.48 & 0.44 & $69: 31$ & & \\
\hline $\mathrm{NO}_{2}$ & 2.89 & -0.33 & 3.22 & 0.48 & 0.44 & $69: 31$ & $\sim 64: 36$ & $\sim 80: 20$ \\
\hline Furan $^{\mathrm{h}}$ & 13.85 & 0.14 & 13.71 & 2.06 & 0.03 & $97: 3$ & $\sim 97: 3$ & $\sim 97: 3$ \\
\hline
\end{tabular}

${ }^{a}$ Calculated from the MP2 energies for the forward reaction in Scheme 10 in units of kcal $/ \mathrm{mol} .{ }^{b} \mathrm{In} \mathrm{kcal} / \mathrm{mol}, \mathrm{T}=$ $298.15 \mathrm{~K} .{ }^{c} \Delta \mathrm{G}$ multiplied by $0.15 .{ }^{d}$ Calculated from $\Delta \mathrm{G}_{\text {adjusted. }}{ }^{e}$ From $\mathrm{K}_{\text {adjusted. }}{ }^{f}$ Average of mole ratio of 3:2 from different substrates in Table 2 under "equilibrating" conditions. ${ }^{g}$ Average of mol ratio of 29:28 from different substrates in Table 4 under "equilibrating" conditions. ${ }^{\mathrm{h}}$ Computations using 35 . Heck product ratio of $\mathbf{6}$ and 7. Suzuki product ratio of $\mathbf{3 0}$ to $\mathbf{3 1}$.

The variation between the experimental ratios determined from the Heck and Suzuki procedures is a reminder that the assumption of a rapid equilibrium, followed by precisely identical rates for the product-determining step, is only approximate. Nonetheless, this very 
empirical approach to predicting the product ratios for several substituents is surprisingly

good, and we take it as support for the general hypothesis of a nearly-equilibrating

intermediate, where the favored regiochemistry is determined qualitatively by the acidities of the relevant protons.

\section{Palladium compounds}<smiles>CN(C)CCPC1(P)CCPC1c1ccccc1-c1ccccc1</smiles>

$\mathrm{HF} / \mathrm{LANL2DV}+\mathrm{f}$ for Pd, LANL2DV for $\mathrm{I}$, and $6-31 \mathrm{G}(\mathrm{d})$ for $\mathrm{C}, \mathrm{H}, \mathrm{P}, \mathrm{N}$

$\begin{array}{lrrrr}\text { C } & 6.0 & 1.5761211741 & -.0868267211 & -2.6084874285 \\ \mathrm{C} & 6.0 & 1.4943746159 & -.2022072982 & .1771277887 \\ \mathrm{C} & 6.0 & .5423334596 & .5129801182 & -1.9097870039 \\ \mathrm{C} & 6.0 & 2.5808599019 & -.7464557370 & -1.9195109622 \\ \mathrm{C} & 6.0 & 2.5359655339 & -.7963032591 & -.5399531182 \\ \mathrm{C} & 6.0 & .4804913218 & .4661362252 & -.5186734499 \\ \mathrm{H} & 1.0 & -.2183790462 & 1.0394513376 & -2.4578302054 \\ \mathrm{H} & 1.0 & 3.3960753083 & -1.2064296838 & -2.4498026151 \\ \mathrm{H} & 1.0 & 3.3267754793 & -1.2878883059 & -.0002526208 \\ \mathrm{PD} & 46.0 & -1.1179094748 & 1.2953526909 & .3985496548 \\ \mathrm{P} & 15.0 & -3.2449582229 & 2.0189715479 & 1.5653019488 \\ \mathrm{P} & 15.0 & -2.0226094698 & -.8845764202 & .7583898914 \\ \mathrm{C} & 6.0 & 1.4996185400 & -.3127381966 & 1.6682080116 \\ \mathrm{C} & 6.0 & 1.5082538406 & -.5512561197 & 4.5076551351 \\ \mathrm{C} & 6.0 & 1.4030646171 & .8025396454 & 2.4947217577 \\ \mathrm{C} & 6.0 & 1.6244251623 & -1.5449070895 & 2.3001244374 \\ \mathrm{C} & 6.0 & 1.6280110572 & -1.6717243716 & 3.6781608469 \\ \mathrm{C} & 6.0 & 1.4076067641 & .6931510145 & 3.8743760679 \\ \mathrm{H} & 1.0 & 1.3482370223 & 1.7818770413 & 2.0561882241 \\ \mathrm{H} & 1.0 & 1.7150136499 & -2.4364518712 & 1.7035424136 \\ \mathrm{H} & 1.0 & 1.7256301489 & -2.6544122170 & 4.0950852222 \\ \mathrm{H} & 1.0 & 1.3520443370 & 1.5969877886 & 4.4472849925 \\ \mathrm{H} & 1.0 & -2.0729534293 & -1.8197176463 & -.2679743119\end{array}$




$\begin{array}{rrrrr}\mathrm{C} & 6.0 & -3.8018132114 & -.7145297838 & 1.2656934790 \\ \mathrm{H} & 1.0 & -1.4670095342 & -1.6535053892 & 1.7738622149 \\ \mathrm{H} & 1.0 & -4.2817879169 & 2.5511877358 & .8017439182 \\ \mathrm{H} & 1.0 & -3.2903012626 & 2.8843900135 & 2.6527791971 \\ \mathrm{H} & 1.0 & -4.3715349558 & -.5386947044 & .3576934583 \\ \mathrm{C} & 6.0 & -3.9973205862 & .4577149972 & 2.2456103482 \\ \mathrm{H} & 1.0 & -4.1724980238 & -1.6357356383 & 1.6996304845 \\ \mathrm{H} & 1.0 & -3.4917462360 & .2442428818 & 3.1829653620 \\ \mathrm{H} & 1.0 & -5.0492435363 & .5813979349 & 2.4733329761 \\ \mathrm{I} & 53.0 & -.3044916357 & 3.8728724148 & -.0904952587 \\ \mathrm{~N} & 7.0 & 1.4736639766 & -.6731341346 & 5.8987854679 \\ \mathrm{H} & 1.0 & 1.6014166317 & -.0288425025 & -3.6828507265 \\ \mathrm{C} & 6.0 & 1.9882289124 & -1.8931842224 & 6.4841160369 \\ \mathrm{C} & 6.0 & 1.6544265031 & .5254716092 & 6.6898396688 \\ \mathrm{H} & 1.0 & 2.6154375867 & 1.0109646143 & 6.5192348997 \\ \mathrm{H} & 1.0 & 1.5841879035 & .2691786253 & 7.7376276278 \\ \mathrm{H} & 1.0 & .8703331532 & 1.2426833080 & 6.4834225765 \\ \mathrm{H} & 1.0 & 3.0260070806 & -2.0899957620 & 6.2150928904 \\ \mathrm{H} & 1.0 & 1.3923859791 & -2.7470450746 & 6.1864742969 \\ \mathrm{H} & 1.0 & 1.9244095473 & -1.8218537812 & 7.5607852712\end{array}$

ZPE $=.365175$ HARTREE/MOLECULE

B3LYP/ LANL2DV+ $f$ for Pd, LANL2DV for I+ P diffuse + $d$ polarization function, and $6-31 \mathrm{G}(d) \mathrm{C}, \mathrm{H}, \mathrm{N}, \mathrm{P}$

$\begin{array}{lrrrr}\mathrm{C} & 6.0 & 1.5754592010 & -0.0998559861 & -2.6172969998 \\ \mathrm{C} & 6.0 & 1.4584559545 & -0.1900011920 & 0.2011682792 \\ \mathrm{C} & 6.0 & 0.5130073673 & 0.4949329537 & -1.9318553439 \\ \mathrm{C} & 6.0 & 2.5891368886 & -0.7383090603 & -1.9008061168 \\ \mathrm{C} & 6.0 & 2.5260840393 & -0.7762770912 & -0.5112892290 \\ \mathrm{C} & 6.0 & 0.4436234972 & 0.4581443640 & -0.5334079418 \\ \mathrm{H} & 1.0 & -0.2619655301 & 1.0073318752 & -2.4950787550 \\ \mathrm{H} & 1.0 & 3.4290436433 & -1.1931529296 & -2.4206463218 \\ \mathrm{H} & 1.0 & 3.3261586741 & -1.2519082081 & 0.0520820761 \\ \mathrm{PD} & 46.0 & -1.2058408499 & 1.2966668181 & 0.3549250269 \\ \mathrm{P} & 15.0 & -3.2478610058 & 2.0725058263 & 1.3878477567 \\ \mathrm{P} & 15.0 & -2.0150353795 & -0.8093502753 & 0.7930652966 \\ \mathrm{C} & 6.0 & 1.4529226171 & -0.3114470811 & 1.6856541357 \\ \mathrm{C} & 6.0 & 1.5242609304 & -0.5901353566 & 4.5395576205 \\ \mathrm{C} & 6.0 & 1.2458064870 & 0.7877924940 & 2.5358371663 \\ \mathrm{C} & 6.0 & 1.7007736692 & -1.5485311607 & 2.3005812919 \\ \mathrm{C} & 6.0 & 1.7344979059 & -1.6950314724 & 3.6850477669 \\ \mathrm{C} & 6.0 & 1.2856995383 & 0.6587069655 & 3.9226325611 \\ \mathrm{H} & 1.0 & 1.0908667413 & 1.7732563553 & 2.1053630565 \\ \mathrm{H} & 1.0 & 1.8676098811 & -2.4267646227 & 1.6808828199\end{array}$




$\begin{array}{rrrrr}\mathrm{H} & 1.0 & 1.9341830704 & -2.6775816849 & 4.0956406277 \\ \mathrm{H} & 1.0 & 1.1547117458 & 1.5523160146 & 4.5217340890 \\ \mathrm{H} & 1.0 & -2.0553420366 & -1.8142953164 & -0.1977720821 \\ \mathrm{C} & 6.0 & -3.8115842174 & -0.6811867724 & 1.3148633434 \\ \mathrm{H} & 1.0 & -1.4302669516 & -1.5555959922 & 1.8402791520 \\ \mathrm{H} & 1.0 & -4.3022759250 & 2.5581954517 & 0.5785117184 \\ \mathrm{H} & 1.0 & -3.3298890692 & 3.0318325796 & 2.4216110278 \\ \mathrm{H} & 1.0 & -4.4006307758 & -0.6064105391 & 0.3922412521 \\ \mathrm{C} & 6.0 & -4.0333721666 & 0.5658298767 & 2.1922721648 \\ \mathrm{H} & 1.0 & -4.1320366046 & -1.5911335174 & 1.8337867330 \\ \mathrm{H} & 1.0 & -3.5303360647 & 0.4364123206 & 3.1585021352 \\ \mathrm{H} & 1.0 & -5.0988300844 & 0.7163174992 & 2.3935591373 \\ \mathrm{I} & 53.0 & -0.3632192765 & 3.8025062796 & -0.2099689031 \\ \mathrm{~N} & 7.0 & 1.5454455002 & -0.7306834749 & 5.9315609204 \\ \mathrm{H} & 1.0 & 1.6148380735 & -0.0499257447 & -3.7035638897 \\ \mathrm{C} & 6.0 & 2.0861159372 & -1.9609876457 & 6.4875689701 \\ \mathrm{C} & 6.0 & 1.6865448146 & 0.4663513407 & 6.7463065779 \\ \mathrm{H} & 1.0 & 2.6340044009 & 1.0026258735 & 6.5664751366 \\ \mathrm{H} & 1.0 & 1.6449615927 & 0.1879633630 & 7.8026224251 \\ \mathrm{H} & 1.0 & 0.8629795616 & 1.1632056552 & 6.5606225579 \\ \mathrm{H} & 1.0 & 3.1342220903 & -2.1429860719 & 6.1962995785 \\ \mathrm{H} & 1.0 & 1.4903682537 & -2.8247328971 & 6.1715499300 \\ \mathrm{H} & 1.0 & 2.0375465276 & -1.9174741996 & 7.5788221174\end{array}$

Energy $=-1497.94076$ HARTREE

B3LYP/ LANL2DV+ $f$ for Pd, LANL2DV for $I+S \& P$ diffuse $+d \& F$ polarization function, and 6-31G+G(3df) for $P, 6-311+G(2 d)$ for $N, 6-$ $31+G(d)$ for $\mathrm{C}, \mathrm{H}$

Energy $=-1498.04939$ HARTREE

MP2/ LANL2DV $+f$ for Pd, LANL2DV for $I+S \& P$ diffuse $+d \& F$ polarization function, and $6-31 \mathrm{G}+\mathrm{G}(3 d f)$ for $\mathrm{P}, 6-311+\mathrm{G}(2 d)$ for $\mathrm{N}, 6-31+G(d)$ for $\mathrm{C}, \mathrm{H}$ Energy $=-1494.77161$ HARTREE 
<smiles>CN(C)c1ccc(-c2ccccc2)c(P2CCP2P2CCP2)c1</smiles>

$H F / L A N L 2 D V+f$ for $P d$, LANL2DV for $I$, and 6-31G(d) for $C, H, P, N$

$\begin{array}{lrrrr}\mathrm{C} & 6.0 & 1.44773245 & 0.25426918 & -2.64156342 \\ \mathrm{C} & 6.0 & 1.37720633 & -0.00700015 & 0.18075365 \\ \mathrm{C} & 6.0 & 0.39459142 & 0.77061892 & -1.87887645 \\ \mathrm{C} & 6.0 & 2.46314454 & -0.42174131 & -1.95432806 \\ \mathrm{C} & 6.0 & 2.41698194 & -0.53608674 & -0.58231890 \\ \mathrm{C} & 6.0 & 0.35137579 & 0.66076261 & -0.49407148 \\ \mathrm{H} & 1.0 & -0.40035215 & 1.29602027 & -2.36669183 \\ \mathrm{H} & 1.0 & 3.30062604 & -0.83969933 & -2.47647071 \\ \mathrm{H} & 1.0 & 3.22710252 & -1.03991890 & -0.08339660 \\ \mathrm{Pd} & 46.0 & -1.27924490 & 1.40157247 & 0.44400612 \\ \mathrm{P} & 15.0 & -3.43088937 & 2.02024102 & 1.62208390 \\ \mathrm{P} & 15.0 & -2.10162306 & -0.81977135 & 0.76036298 \\ \mathrm{C} & 6.0 & 1.40311861 & -0.18225352 & 1.66406178 \\ \mathrm{C} & 6.0 & 1.45767593 & -0.53985649 & 4.44655800 \\ \mathrm{C} & 6.0 & 1.25869775 & 0.90260822 & 2.52933455 \\ \mathrm{C} & 6.0 & 1.59046054 & -1.44546843 & 2.22550511 \\ \mathrm{C} & 6.0 & 1.61818552 & -1.62437308 & 3.59962034 \\ \mathrm{C} & 6.0 & 1.28134739 & 0.72369140 & 3.90417480 \\ \mathrm{H} & 1.0 & 1.15432024 & 1.89118803 & 2.12231255 \\ \mathrm{H} & 1.0 & 1.70736492 & -2.29693675 & 1.57787740 \\ \mathrm{H} & 1.0 & 1.76390064 & -2.60994411 & 4.00642061 \\ \mathrm{H} & 1.0 & 1.17984784 & 1.57780182 & 4.55057669 \\ \mathrm{H} & 1.0 & -2.12437725 & -1.73310375 & -0.28633744 \\ \mathrm{C} & 6.0 & -3.88416815 & -0.72903663 & 1.27943861 \\ \mathrm{H} & 1.0 & -1.51044452 & -1.59006369 & 1.75449371 \\ \mathrm{H} & 1.0 & -4.48494625 & 2.52827930 & 0.86562324 \\ \mathrm{H} & 1.0 & -3.50758481 & 2.86418414 & 2.72440958 \\ \mathrm{H} & 1.0 & -4.46617126 & -0.56735557 & 0.37664020 \\ \mathrm{C} & 6.0 & -4.12582541 & 0.42126784 & 2.27492762 \\ \mathrm{H} & 1.0 & -4.21293926 & -1.67035627 & 1.70412898 \\ \mathrm{H} & 1.0 & -3.61488795 & 0.21354850 & 3.21063542 \\ \mathrm{H} & 1.0 & -5.18232632 & 0.50170761 & 2.50112844 \\ \mathrm{I} & 53.0 & -0.57620537 & 4.02269983 & 0.01963981 \\ \mathrm{H} & 1.0 & 1.48178446 & -0.67542797 & 5.51338482 \\ \mathrm{~N} & 7.0 & 1.50186789 & 0.42002988 & -4.02309275 \\ \mathrm{C} & 6.0 & 2.32352710 & -0.48873413 & -4.79189730 \\ \mathrm{C} & 6.0 & 0.35875925 & 0.98589790 & -4.70165300 \\ \mathrm{H} & 1.0 & 0.59907955 & 1.11488044 & -5.74800825\end{array}$




$\begin{array}{rrrrr}\mathrm{H} & 1.0 & 0.12362382 & 1.96340621 & -4.30202436 \\ \mathrm{H} & 1.0 & -0.53434920 & 0.36207941 & -4.63188124 \\ \mathrm{H} & 1.0 & 3.37278247 & -0.36694849 & -4.55209303 \\ \mathrm{H} & 1.0 & 2.20957518 & -0.26486143 & -5.84323120 \\ \mathrm{H} & 1.0 & 2.05781746 & -1.53464985 & -4.63558626\end{array}$

ZPE $=0.365233$ HARTREE/MOLECULE

B3LYP/ LANL2DV+ $f$ for Pd, LANL2DV for I+ P diffuse + $d$ polarization function, and $6-31 \mathrm{G}(d) \mathrm{C}, \mathrm{H}, \mathrm{N}, \mathrm{P}$

$\begin{array}{lrrrr}\mathrm{C} & 6.0 & 1.41484833 & 0.22364886 & -2.64665151 \\ \mathrm{C} & 6.0 & 1.38282824 & -0.02744289 & 0.20947140 \\ \mathrm{C} & 6.0 & 0.37587425 & 0.77576643 & -1.86039269 \\ \mathrm{C} & 6.0 & 2.42537332 & -0.49031410 & -1.96416402 \\ \mathrm{C} & 6.0 & 2.40064359 & -0.59669781 & -0.58057076 \\ \mathrm{C} & 6.0 & 0.36032632 & 0.66600275 & -0.47113574 \\ \mathrm{H} & 1.0 & -0.42898706 & 1.31785798 & -2.33979058 \\ \mathrm{H} & 1.0 & 3.24581456 & -0.94538081 & -2.50727034 \\ \mathrm{H} & 1.0 & 3.21249652 & -1.12492073 & -0.08437874 \\ \mathrm{Pd} & 46.0 & -1.30315626 & 1.41535878 & 0.47938609 \\ \mathrm{P} & 15.0 & -3.37320518 & 2.04069185 & 1.55934536 \\ \mathrm{P} & 15.0 & -2.04044914 & -0.74082190 & 0.75059462 \\ \mathrm{C} & 6.0 & 1.41034102 & -0.19981304 & 1.68575191 \\ \mathrm{C} & 6.0 & 1.44331646 & -0.55567986 & 4.49137545 \\ \mathrm{C} & 6.0 & 1.15358257 & 0.87699747 & 2.55537796 \\ \mathrm{C} & 6.0 & 1.69800949 & -1.45343316 & 2.25667334 \\ \mathrm{C} & 6.0 & 1.71402323 & -1.63063037 & 3.64144182 \\ \mathrm{C} & 6.0 & 1.16756392 & 0.69864845 & 3.94079494 \\ \mathrm{H} & 1.0 & 0.97682571 & 1.86551034 & 2.14169765 \\ \mathrm{H} & 1.0 & 1.89371049 & -2.30099702 & 1.60429919 \\ \mathrm{H} & 1.0 & 1.93354905 & -2.61225677 & 4.05506945 \\ \mathrm{H} & 1.0 & 0.98123521 & 1.55154645 & 4.58924818 \\ \mathrm{H} & 1.0 & -2.04796171 & -1.65866470 & -0.32151207 \\ \mathrm{C} & 6.0 & -3.84185410 & -0.71742004 & 1.27416945 \\ \mathrm{H} & 1.0 & -1.43082309 & -1.55068469 & 1.73415911 \\ \mathrm{H} & 1.0 & -4.43925571 & 2.54615974 & 0.77795678 \\ \mathrm{H} & 1.0 & -3.49711227 & 2.91928792 & 2.65888810 \\ \mathrm{H} & 1.0 & -4.43266153 & -0.59582597 & 0.35768244 \\ \mathrm{C} & 6.0 & -4.10929871 & 0.45173532 & 2.24162436 \\ \mathrm{H} & 1.0 & -4.12879896 & -1.67431378 & 1.72298992 \\ \mathrm{H} & 1.0 & -3.60773015 & 0.26599568 & 3.19956064 \\ \mathrm{H} & 1.0 & -5.18021727 & 0.55084515 & 2.44594669 \\ \mathrm{I} & 53.0 & -0.55039501 & 3.98203802 & 0.05632673 \\ \mathrm{H} & 1.0 & 1.45855999 & -0.69245297 & 5.56968355 \\ \mathrm{~N} & 7.0 & 1.45042431 & 0.39159665 & -4.02856779 \\ & & & & \\ \mathrm{H} & 1.0 & & \end{array}$




$\begin{array}{rrrrr}\mathrm{C} & 6.0 & 2.30754876 & -0.47414604 & -4.82136917 \\ \mathrm{C} & 6.0 & 0.31366745 & 0.99761450 & -4.70047665 \\ \mathrm{H} & 1.0 & 0.56162524 & 1.14233410 & -5.75539684 \\ \mathrm{H} & 1.0 & 0.09472293 & 1.98257911 & -4.27557516 \\ \mathrm{H} & 1.0 & -0.60368448 & 0.38642254 & -4.64093971 \\ \mathrm{H} & 1.0 & 3.36130834 & -0.34866977 & -4.54697704 \\ \mathrm{H} & 1.0 & 2.20901179 & -0.20202255 & -5.87454128 \\ \mathrm{H} & 1.0 & 2.05452132 & -1.54288220 & -4.71522760\end{array}$

Energy $=-1497.93652$ Hartree

B3LYP/ LANL2DV+ $f$ for $P d$, LANL2DV for $I+S \& P$ diffuse $+d \& F$ polarization function, and $6-31 G+G(3 d f)$ for $P, 6-311+G(2 d)$ for $N, 6-$ $31+G(d)$ for $\mathrm{C}, \mathrm{H}$

Energy $=-1498.049164$ HARTREE

MP2/ LANL2DV+ $f$ for Pd, LANL2DV for $I+S \& P$ diffuse $+d \& F$ polarization function, and $6-31 \mathrm{G}+\mathrm{G}(3 d f)$ for $\mathrm{P}, 6-311+\mathrm{G}(2 d)$ for $\mathrm{N}, 6-31+G(d)$ for $\mathrm{C}, \mathrm{H}$ Energy $=-1494.77128$ HARTREE<smiles>O=[N+]([O-])c1ccc(-c2ccccc2P2CCP2CCP)cc1</smiles>

HF/LANL2DV $+f$ for Pd, LANL2DV for $I$, and 6-31G(d) for $C, H, P, N, 0$

$\begin{array}{lrrrr}\mathrm{H} & 1.0 & 1.83590925 & 0.31626487 & -5.04355764 \\ \mathrm{C} & 6.0 & 1.80174220 & 0.25801694 & -3.96980858 \\ \mathrm{C} & 6.0 & 1.68994141 & 0.13958621 & -1.19569504 \\ \mathrm{C} & 6.0 & 0.65913779 & 0.65224576 & -3.29577851 \\ \mathrm{C} & 6.0 & 2.90216827 & -0.19804907 & -3.26142812 \\ \mathrm{C} & 6.0 & 2.83964443 & -0.25563508 & -1.88409829 \\ \mathrm{C} & 6.0 & 0.57732576 & 0.60537457 & -1.90450597 \\ \mathrm{H} & 1.0 & -0.17540210 & 1.02196658 & -3.86405182 \\ \mathrm{H} & 1.0 & 3.79869127 & -0.49659562 & -3.77463818 \\ \mathrm{H} & 1.0 & 3.69663405 & -0.59223700 & -1.32705629 \\ \mathrm{Pd} & 46.0 & -1.17562175 & 1.13444316 & -1.03465891 \\ \mathrm{P} & 15.0 & -3.41714334 & 1.51052380 & 0.04692559 \\ \mathrm{P} & 15.0 & -1.73743927 & -1.16637778 & -0.69561845\end{array}$




$\begin{array}{rrrrr}\mathrm{C} & 6.0 & 1.69614410 & 0.03956110 & 0.29509276 \\ \mathrm{C} & 6.0 & 1.69436419 & -0.18391101 & 3.05673003 \\ \mathrm{C} & 6.0 & 1.38031757 & 1.13712621 & 1.09746075 \\ \mathrm{C} & 6.0 & 2.03396344 & -1.16407204 & 0.91646940 \\ \mathrm{C} & 6.0 & 2.03413558 & -1.28573275 & 2.29282093 \\ \mathrm{C} & 6.0 & 1.37606728 & 1.03143311 & 2.47629666 \\ \mathrm{H} & 1.0 & 1.15586042 & 2.08097935 & 0.63913387 \\ \mathrm{H} & 1.0 & 2.28769588 & -2.01675677 & 0.31358474 \\ \mathrm{H} & 1.0 & 2.28499603 & -2.21218538 & 2.76857734 \\ \mathrm{H} & 1.0 & 1.14072049 & 1.87586975 & 3.09227014 \\ \mathrm{~N} & 7.0 & 1.67108226 & -0.30886975 & 4.50624275 \\ \mathrm{H} & 1.0 & -1.61102438 & -2.09128666 & -1.72431076 \\ \mathrm{C} & 6.0 & -3.53317308 & -1.27836967 & -0.23185147 \\ \mathrm{H} & 1.0 & -1.09042239 & -1.84494352 & 0.33127633 \\ \mathrm{H} & 1.0 & -4.49243927 & 1.87399006 & -0.75982362 \\ \mathrm{H} & 1.0 & -3.62047982 & 2.37123609 & 1.11885953 \\ \mathrm{H} & 1.0 & -4.09990931 & -1.20953548 & -1.15586972 \\ \mathrm{C} & 6.0 & -3.94124818 & -0.14170122 & 0.72407663 \\ \mathrm{H} & 1.0 & -3.75938201 & -2.24230433 & 0.20846392 \\ \mathrm{H} & 1.0 & -3.44292402 & -0.26486653 & 1.68126857 \\ \mathrm{H} & 1.0 & -5.00695753 & -0.18089488 & 0.91449648 \\ \mathrm{I} & 53.0 & -0.76935738 & 3.80298257 & -1.47910476 \\ \mathrm{O} & 8.0 & 1.36202645 & 0.65255523 & 5.14363480 \\ \mathrm{O} & 8.0 & 1.95435488 & -1.36983120 & 4.97817564\end{array}$

ZPE $=.290601$ HARTREE/MOLECULE

B3LYP/ LANL2DV $+f$ for Pd, LANL2DV for $I+P$ diffuse $+d$ polarization function, and $6-31 \mathrm{G}(\mathrm{d}) \mathrm{C}, \mathrm{H}, \mathrm{N}, \mathrm{P}, \mathrm{O}$

$\begin{array}{lrrrr}\mathrm{H} & 1.0 & 1.84882796 & 0.28833732 & -5.08015442 \\ \mathrm{C} & 6.0 & 1.80438209 & 0.23967749 & -3.99430704 \\ \mathrm{C} & 6.0 & 1.66819501 & 0.14923745 & -1.18715119 \\ \mathrm{C} & 6.0 & 0.64108288 & 0.63483942 & -3.32901263 \\ \mathrm{C} & 6.0 & 2.90922070 & -0.20076497 & -3.26297927 \\ \mathrm{C} & 6.0 & 2.83488107 & -0.24462062 & -1.87513125 \\ \mathrm{C} & 6.0 & 0.55624801 & 0.59919101 & -1.93070793 \\ \mathrm{H} & 1.0 & -0.20475151 & 0.99310315 & -3.90922666 \\ \mathrm{H} & 1.0 & 3.82319403 & -0.49996325 & -3.76949072 \\ \mathrm{H} & 1.0 & 3.69984889 & -0.56666166 & -1.29936147 \\ \mathrm{Pd} & 46.0 & -1.22794557 & 1.11059797 & -1.05530894 \\ \mathrm{P} & 15.0 & -3.35907125 & 1.54466963 & -0.02077933 \\ \mathrm{P} & 15.0 & -1.74135005 & -1.11565423 & -0.71420139 \\ \mathrm{C} & 6.0 & 1.66762626 & 0.04177174 & 0.29692084 \\ \mathrm{C} & 6.0 & 1.69399559 & -0.22359370 & 3.07956934 \\ \mathrm{C} & 6.0 & 1.23989320 & 1.09943175 & 1.12411964 \\ \mathrm{C} & 6.0 & 2.12291312 & -1.14246631 & 0.90870631\end{array}$




$\begin{array}{rrrrr}\mathrm{C} & 6.0 & 2.13815308 & -1.28569317 & 2.29182315 \\ \mathrm{C} & 6.0 & 1.25166428 & 0.97157735 & 2.51171374 \\ \mathrm{H} & 1.0 & 0.92666954 & 2.03866625 & 0.67822975 \\ \mathrm{H} & 1.0 & 2.45356178 & -1.96866286 & 0.28583366 \\ \mathrm{H} & 1.0 & 2.47830224 & -2.19866037 & 2.76578498 \\ \mathrm{H} & 1.0 & 0.93865883 & 1.78554285 & 3.15468335 \\ \mathrm{~N} & 7.0 & 1.69504166 & -0.36985409 & 4.53990459 \\ \mathrm{H} & 1.0 & -1.65532565 & -2.06374621 & -1.75617886 \\ \mathrm{C} & 6.0 & -3.53420758 & -1.25241303 & -0.18407196 \\ \mathrm{H} & 1.0 & -1.04632008 & -1.82856166 & 0.28836781 \\ \mathrm{H} & 1.0 & -4.46186829 & 1.91462362 & -0.82518446 \\ \mathrm{H} & 1.0 & -3.55464840 & 2.45720387 & 1.03797936 \\ \mathrm{H} & 1.0 & -4.13242006 & -1.23401403 & -1.10361302 \\ \mathrm{C} & 6.0 & -3.92766666 & -0.07802179 & 0.73202080 \\ \mathrm{H} & 1.0 & -3.72232080 & -2.21376014 & 0.30569547 \\ \mathrm{H} & 1.0 & -3.41443586 & -0.16683921 & 1.69728482 \\ \mathrm{H} & 1.0 & -5.00405979 & -0.08398663 & 0.93028605 \\ \mathrm{I} & 53.0 & -0.79172438 & 3.72674036 & -1.55450487 \\ \mathrm{O} & 8.0 & 1.28295648 & 0.57665920 & 5.21274137 \\ \mathrm{O} & 8.0 & 2.10279822 & -1.43393266 & 5.00970030\end{array}$

Energy $=-1568.48222$ HARTREE

B3LYP/ LANL2DV+ $f$ for Pd, LANL2DV for $I+S \& P$ diffuse $+d \& F$ polarization function, and $6-31 \mathrm{G}+\mathrm{G}(3 d f)$ for $P, 6-311+G(2 d)$ for $N \& 0$, 6$31+\mathrm{G}(d)$ for $\mathrm{C}, \mathrm{H}$

Energy $=-1568.64625$ HARTREE

MP2/ LANL2DV $+f$ for Pd, LANL2DV for I+ $S$ \& P diffuse $+d$ \& F polarization function, and $6-31 \mathrm{G}+\mathrm{G}(3 d f)$ for $P, 6-311+G(2 d)$ for $N \& 0,6-31+G(d)$ for $C, H$ Energy $=-1565.39048$ HARTREE<smiles>O=[N+]([O-])c1ccc(-c2ccccc2)c(-c2ccccc2)c1</smiles>

HF/LANL2DV+ $f$ for Pd, LANL2DV for I, and 6-31G(d) for $C, H, P, N, 0$
$\mathrm{N} \quad 7.0$
1.87952793
0.31807858
$-4.77727938$
C $\quad 6.0$
1.82459283
0.25905609
$-3.32140255$ 


$\begin{array}{lrrrr}\mathrm{C} & 6.0 & 1.68870652 & 0.11257151 & -0.57708889 \\ \mathrm{C} & 6.0 & 0.66495848 & 0.64262748 & -2.67654395 \\ \mathrm{C} & 6.0 & 2.93750286 & -0.18351431 & -2.63036704 \\ \mathrm{C} & 6.0 & 2.85413337 & -0.25362638 & -1.25723207 \\ \mathrm{C} & 6.0 & 0.57646531 & 0.57770073 & -1.28828573 \\ \mathrm{H} & 1.0 & -0.15703757 & 0.99962264 & -3.26306987 \\ \mathrm{H} & 1.0 & 3.83013701 & -0.45895341 & -3.15378714 \\ \mathrm{H} & 1.0 & 3.70895600 & -0.58476299 & -0.69612616 \\ \mathrm{Pd} & 46.0 & -1.17857504 & 1.10075486 & -0.41100559 \\ \mathrm{P} & 15.0 & -3.40291333 & 1.49562716 & 0.65940362 \\ \mathrm{P} & 15.0 & -1.76317871 & -1.19743586 & -0.08562494 \\ \mathrm{C} & 6.0 & 1.67116249 & -0.02642692 & 0.91266620 \\ \mathrm{C} & 6.0 & 1.63798940 & -0.32156527 & 3.69382644 \\ \mathrm{C} & 6.0 & 1.36460960 & 1.05356824 & 1.73813891 \\ \mathrm{C} & 6.0 & 1.97650445 & -1.24995518 & 1.50535595 \\ \mathrm{C} & 6.0 & 1.95581019 & -1.39917076 & 2.88311005 \\ \mathrm{C} & 6.0 & 1.34866250 & 0.90499055 & 3.11657596 \\ \mathrm{H} & 1.0 & 1.16025984 & 2.01336861 & 1.30180550 \\ \mathrm{H} & 1.0 & 2.22415996 & -2.09231853 & 0.88326627 \\ \mathrm{H} & 1.0 & 2.19069409 & -2.35346293 & 3.32048106 \\ \mathrm{H} & 1.0 & 1.12282932 & 1.75340950 & 3.73778009 \\ \mathrm{H} & 1.0 & -1.64128244 & -2.12206244 & -1.11522079 \\ \mathrm{C} & 6.0 & -3.56326389 & -1.28804660 & 0.36597028 \\ \mathrm{H} & 1.0 & -1.12879360 & -1.88131666 & 0.94502455 \\ \mathrm{H} & 1.0 & -4.46078014 & 1.88361061 & -0.15807471 \\ \mathrm{H} & 1.0 & -3.59534073 & 2.35745263 & 1.73213637 \\ \mathrm{H} & 1.0 & -4.12367392 & -1.20781982 & -0.56085628 \\ \mathrm{C} & 6.0 & -3.96129632 & -0.14971064 & 1.32433319 \\ \mathrm{H} & 1.0 & -3.80476975 & -2.25095081 & 0.80030191 \\ \mathrm{H} & 1.0 & -3.47316837 & -0.28594047 & 2.28503251 \\ \mathrm{H} & 1.0 & -5.02916813 & -0.17051524 & 1.50509131 \\ \mathrm{I} & 53.0 & -0.76402712 & 3.76871490 & -0.83196586 \\ \mathrm{H} & 1.0 & 1.62751293 & -0.43328258 & 4.76340914 \\ \mathrm{O} & 8.0 & 2.92690158 & 0.08462854 & -5.30383301 \\ \mathrm{O} & 8.0 & 0.87518418 & 0.58505493 & -5.36594534 \\ & & & & \\ \mathrm{H} & 1.0 & & \end{array}$

ZPE $=.290494$ HARTREE/MOLECULE

B3LYP/ LANL2DV $+f$ for Pd, LANL2DV for $I+P$ diffuse + d polarization function, and 6-31G(d) $\mathrm{C}, \mathrm{H}, \mathrm{N}, \mathrm{P}, \mathrm{O}$

$\begin{array}{lllrr}\mathrm{N} & 7.0 & 1.88150203 & 0.29587021 & -4.80446672 \\ \mathrm{C} & 6.0 & 1.81862533 & 0.24828310 & -3.33589220 \\ \mathrm{C} & 6.0 & 1.66785121 & 0.12117074 & -0.55857420 \\ \mathrm{C} & 6.0 & 0.64352316 & 0.63855755 & -2.69434738 \\ \mathrm{C} & 6.0 & 2.93547702 & -0.19048598 & -2.62694812\end{array}$




\begin{tabular}{|c|c|c|c|c|}
\hline C & 6.0 & 2.84457707 & -0.24906917 & -1.24341869 \\
\hline C & 6.0 & 0.55657977 & 0.58206183 & -1.29968464 \\
\hline $\mathrm{H}$ & 1.0 & -0.18608323 & 0.98816043 & -3.29657722 \\
\hline $\mathrm{H}$ & 1.0 & 3.83805728 & -0.47028559 & -3.15626931 \\
\hline $\mathrm{H}$ & 1.0 & 3.70600843 & -0.57595527 & -0.66669005 \\
\hline $\mathrm{Pd}$ & 46.0 & -1.22523022 & 1.08550656 & -0.41311386 \\
\hline$P$ & 15.0 & -3.34660697 & 1.51670408 & 0.62057340 \\
\hline$P$ & 15.0 & -1.76126659 & -1.14319122 & -0.12677138 \\
\hline C & 6.0 & 1.64723754 & -0.02641483 & 0.92445856 \\
\hline C & 6.0 & 1.63930130 & -0.35538608 & 3.72368264 \\
\hline C & 6.0 & 1.24969208 & 1.02596498 & 1.76764953 \\
\hline C & 6.0 & 2.04994416 & -1.23930168 & 1.50952446 \\
\hline C & 6.0 & 2.04165697 & -1.40559912 & 2.89532518 \\
\hline C & 6.0 & 1.24988091 & 0.85992831 & 3.15515733 \\
\hline $\mathrm{H}$ & 1.0 & 0.97503895 & 1.98501885 & 1.33824503 \\
\hline $\mathrm{H}$ & 1.0 & 2.36032057 & -2.06197548 & 0.86999995 \\
\hline $\mathrm{H}$ & 1.0 & 2.35266209 & -2.35431099 & 3.32562709 \\
\hline $\mathrm{H}$ & 1.0 & 0.95801598 & 1.69206703 & 3.79089856 \\
\hline $\mathrm{H}$ & 1.0 & -1.67722762 & -2.07217407 & -1.18586695 \\
\hline C & 6.0 & -3.55738497 & -1.27302873 & 0.39223185 \\
\hline $\mathrm{H}$ & 1.0 & -1.07441294 & -1.87491894 & 0.86707717 \\
\hline $\mathrm{H}$ & 1.0 & -4.43656063 & 1.91994822 & -0.18450722 \\
\hline $\mathrm{H}$ & 1.0 & -3.53443408 & 2.40946794 & 1.69723153 \\
\hline $\mathrm{H}$ & 1.0 & -4.15124273 & -1.22824693 & -0.52900660 \\
\hline C & 6.0 & -3.94102192 & -0.11492360 & 1.33307719 \\
\hline $\mathrm{H}$ & 1.0 & -3.75748205 & -2.24259567 & 0.86040622 \\
\hline $\mathrm{H}$ & 1.0 & -3.43354368 & -0.23270389 & 2.29828048 \\
\hline $\mathrm{H}$ & 1.0 & -5.01857233 & -0.11093161 & 1.52486980 \\
\hline I & 53.0 & -0.77029771 & 3.71386194 & -0.81516892 \\
\hline $\mathrm{H}$ & 1.0 & 1.64189863 & -0.48019639 & 4.80337238 \\
\hline 0 & 8.0 & 2.94819808 & -0.00789135 & -5.34282064 \\
\hline 0 & 8.0 & 0.86531031 & 0.62701499 & -5.41756392 \\
\hline
\end{tabular}

B3LYP/ LANL2DV+ $f$ for Pd, LANL2DV for $I+S \& P$ diffuse $+d \& F$ polarization function, and $6-31 \mathrm{G}+\mathrm{G}(3 d f)$ for $\mathrm{P}, 6-311+G(2 d)$ for $N \& 0,6-$ $31+\mathrm{G}(d)$ for $\mathrm{C}, \mathrm{H}$

Energy $=-1568.64722$ HARTREE 


\begin{tabular}{|c|c|c|c|c|}
\hline \multicolumn{5}{|c|}{ Anions } \\
\hline \multicolumn{5}{|c|}{ 2-carbanion of 4-hydroxybiphenyl } \\
\hline \multicolumn{5}{|c|}{$H F / 6-31+G(d, p)$} \\
\hline C & 6.0 & .0610065004 & .0241960470 & -.9372952832 \\
\hline C & 6.0 & .0928247308 & .0348147732 & .5586221194 \\
\hline C & 6.0 & .0379155870 & .0464054185 & 3.3223186319 \\
\hline $\mathrm{C}$ & 6.0 & 1.2974712792 & .3965322291 & 1.2239099218 \\
\hline C & 6.0 & -1.0632448588 & -.3257591675 & 1.2532353563 \\
\hline C & 6.0 & -1.1101153256 & -.3249900718 & 2.6415973089 \\
\hline $\mathrm{C}$ & 6.0 & 1.1898607292 & .3868006948 & 2.6274184516 \\
\hline $\mathrm{H}$ & 1.0 & -1.9479997499 & -.6411414182 & .7217349121 \\
\hline $\mathrm{H}$ & 1.0 & -1.9958923221 & -.6138596390 & 3.1810325419 \\
\hline $\mathrm{H}$ & 1.0 & 2.0573319721 & .6567142584 & 3.2255654640 \\
\hline 0 & 8.0 & -.0048693711 & .0521642572 & 4.6950216524 \\
\hline C & 6.0 & .0450437428 & -.0367767726 & -3.7678628446 \\
\hline C & 6.0 & -1.0973317767 & .2973049648 & -1.6723750277 \\
\hline C & 6.0 & 1.2205837320 & -.2614992413 & -1.6715550427 \\
\hline C & 6.0 & 1.2122323873 & -.2951725707 & -3.0551744701 \\
\hline C & 6.0 & -1.1086272086 & .2638198480 & -3.0619113738 \\
\hline $\mathrm{H}$ & 1.0 & -2.0014456235 & .5660429340 & -1.1555604358 \\
\hline $\mathrm{H}$ & 1.0 & 2.1259846993 & -.4424418786 & -1.1253437020 \\
\hline $\mathrm{H}$ & 1.0 & 2.1228070674 & -.5245114188 & -3.5847986501 \\
\hline $\mathrm{H}$ & 1.0 & -2.0215370255 & .4866976664 & -3.5902917934 \\
\hline $\mathrm{H}$ & 1.0 & .0408236203 & -.0603531836 & -4.8448884374 \\
\hline $\mathrm{H}$ & 1.0 & .8457692861 & .3138300921 & 5.0182088693 \\
\hline
\end{tabular}

Energy

ZPE

$M P 2 / 6-311+G(d, p)$

$B 3 L Y P / 6-311+G(d, p)$

-534.4802491009 Hartree .183084 HARTREE/MOLECULE -536.4717137938 Hartree

-537.7079700998 Hartree

2'-carbanion of 4-hydroxybiphenyl

$H F / 6-31+G(d, p)$

$\begin{array}{lrrrr}C & 6.0 & .0460082284 & .0580955637 & -.4315921461 \\ \mathrm{C} & 6.0 & .0757371982 & .0690723435 & 1.0688274136 \\ \mathrm{C} & 6.0 & .0179896902 & .0631129537 & 3.8359843438 \\ \mathrm{C} & 6.0 & 1.1473677147 & .7273890477 & 1.7253041128 \\ \mathrm{C} & 6.0 & -.9547638296 & -.5847291673 & 1.7553579613 \\ \mathrm{C} & 6.0 & -.9950416660 & -.5898962185 & 3.1423011924 \\ \mathrm{C} & 6.0 & 1.0448998765 & .6852965303 & 3.1340226209 \\ \mathrm{H} & 1.0 & -1.7247882686 & -1.1164693694 & 1.2166815492\end{array}$




$\begin{array}{rrrrr}\mathrm{H} & 1.0 & -1.7885631143 & -1.1000677544 & 3.6657322220 \\ \mathrm{H} & 1.0 & 1.8154846394 & 1.1677953059 & 3.7232644160 \\ \mathrm{H} & 1.0 & .0078900351 & .0745151925 & 4.9171463339 \\ \mathrm{C} & 6.0 & .0498429248 & .0267156206 & -3.2558906994 \\ \mathrm{C} & 6.0 & -1.1361490872 & .0407663425 & -1.1735477144 \\ \mathrm{C} & 6.0 & 1.2354781560 & .0779594527 & -1.1640208173 \\ \mathrm{C} & 6.0 & 1.2435438304 & .0579212735 & -2.5502492808 \\ \mathrm{C} & 6.0 & -1.1451780722 & .0221661967 & -2.5642568973 \\ \mathrm{H} & 1.0 & -2.0820044217 & .0665368580 & -.6625350827 \\ \mathrm{H} & 1.0 & 2.1583199141 & .1239956122 & -.6189744509 \\ \mathrm{H} & 1.0 & 2.1843548796 & .0675658114 & -3.0806571728 \\ \mathrm{H} & 1.0 & -2.0724192104 & .0173263697 & -3.1106774506 \\ \mathrm{O} & 8.0 & .0121251448 & .0064938319 & -4.6258793934 \\ \mathrm{C} & 1.0 & .8930696008 & .0502783260 & -4.9684917752\end{array}$

Energy

ZPE

$M P 2 / 6-311+G(d, p)$

$B 3 L Y P / 6-311+G(d, p)$

-534.4735035275 Hartree .182774 HARTREE/MOLECULE $-536.4674726383$

$-537.7022560781$

2-carbanion of 4-nitrobiphenyl

$H F / 6-31+G(d, p)$

$\begin{array}{lrrrr}\mathrm{C} & 6.0 & -0.0017277833 & -0.1428960045 & -0.3140383338 \\ \mathrm{C} & 6.0 & -0.0125682442 & -0.1036462691 & 1.1848109358 \\ \mathrm{C} & 6.0 & 0.0011204135 & -0.0691952787 & 4.0044763099 \\ \mathrm{C} & 6.0 & 0.5075114763 & -1.1731988401 & 1.9212991453 \\ \mathrm{C} & 6.0 & -0.5393571361 & 0.9719842457 & 1.9023464206 \\ \mathrm{C} & 6.0 & -0.5340757290 & 0.9915633038 & 3.2919722029 \\ \mathrm{C} & 6.0 & 0.5202128057 & -1.1538645370 & 3.3056762985 \\ \mathrm{C} & 1.0 & 0.8857312188 & -2.0191008204 & 1.3809324217 \\ \mathrm{H} & 1.0 & -0.9849219629 & 1.7962353343 & 1.3745774793 \\ \mathrm{H} & 1.0 & -0.9586230112 & 1.8335280812 & 3.8133700264 \\ \mathrm{H} & 1.0 & 0.9331277511 & -1.9907522170 & 3.8441691171 \\ \mathrm{H} & 1.0 & 0.0068182775 & -0.0578324090 & 5.0814162406 \\ \mathrm{C} & 6.0 & -0.0181822892 & -0.0902641996 & -3.0485623260 \\ \mathrm{C} & 6.0 & 0.1463814602 & 1.0746159330 & -1.0014622479 \\ \mathrm{C} & 6.0 & -0.1303600861 & -1.3926928540 & -0.9603127402 \\ \mathrm{C} & 6.0 & -0.1362006339 & -1.2947830010 & -2.3702050685 \\ \mathrm{C} & 6.0 & 0.1369073371 & 1.1184819090 & -2.3787740908 \\ \mathrm{H} & 1.0 & 0.2937509928 & 1.9972779345 & -0.4646654480 \\ \mathrm{H} & 1.0 & -0.2366875599 & -2.1875301013 & -2.9603515846 \\ \mathrm{H} & 1.0 & 0.2546247171 & 2.0388440560 & -2.9148626018 \\ \mathrm{~N} & 7.0 & -0.0384755303 & -0.0675622226 & -4.5059799772 \\ \mathrm{O} & 8.0 & 0.0591629675 & 0.9942442451 & -5.0650981513 \\ \mathrm{O} & 8.0 & -0.1516275505 & -1.1026337854 & -5.1006399588\end{array}$


Energy

ZPE

$M P 2 / 6-311+G(d, p)$

$B 3 L Y P / 6-311+G(d, p)$

2'-carbanion of 4-nitrobiphenyl

$H F / 6-31+G(d, p)$

$\begin{array}{lrrrr}\mathrm{C} & 6.0 & 0.0469790907 & -0.0839666425 & -0.1865668558 \\ \mathrm{C} & 6.0 & 0.0453594275 & -0.1161833850 & 1.3020413278 \\ \mathrm{C} & 6.0 & 0.0526405396 & -0.0749364609 & 4.0639385268 \\ \mathrm{C} & 6.0 & 0.5329568334 & -1.2781198578 & 1.9490177644 \\ \mathrm{C} & 6.0 & -0.4441280122 & 1.0047840003 & 1.9952295340 \\ \mathrm{C} & 6.0 & -0.4470328172 & 1.0323142041 & 3.3761567922 \\ \mathrm{C} & 6.0 & 0.5153574160 & -1.1759065499 & 3.3621400582 \\ \mathrm{H} & 1.0 & -0.8528667324 & 1.8502971295 & 1.4648556695 \\ \mathrm{H} & 1.0 & -0.8363400879 & 1.8859091912 & 3.9071576644 \\ \mathrm{H} & 1.0 & 0.8798756901 & -2.0120382897 & 3.9448049768 \\ \mathrm{H} & 1.0 & 0.0657691442 & -0.0703574874 & 5.1445529240 \\ \mathrm{C} & 6.0 & 0.0235592153 & -0.0828376078 & -2.9886645809 \\ \mathrm{C} & 6.0 & 0.1082391223 & 1.1109296628 & -0.9231260185 \\ \mathrm{C} & 6.0 & -0.0039714295 & -1.2835608852 & -0.9204044926 \\ \mathrm{C} & 6.0 & -0.0212467938 & -1.2905746454 & -2.2960645374 \\ \mathrm{C} & 6.0 & 0.0947792184 & 1.1202075374 & -2.3037137638 \\ \mathrm{H} & 1.0 & 0.1956319125 & 2.0496293982 & -0.4099987949 \\ \mathrm{H} & 1.0 & -0.0182009162 & -2.2021246792 & -0.3690762433 \\ \mathrm{H} & 1.0 & -0.0707221250 & -2.2148110580 & -2.8374019567 \\ \mathrm{H} & 1.0 & 0.1482047908 & 2.0435698565 & -2.8459866528 \\ \mathrm{~N} & 7.0 & 0.0044329290 & -0.0801533808 & -4.4300314259 \\ \mathrm{O} & 8.0 & 0.0475996138 & 0.9766454664 & -5.0020105802 \\ \mathrm{O} & 8.0 & -0.0553751634 & -1.1336153606 & -5.0035633742\end{array}$

Energy

ZPE

$M P 2 / 6-311+G(d, p)$

$B 3 L Y P / 6-311+G(d, p)$

2-carbanion of 4-carbomethoxybiphenyl

$H F / 6-31+G(d, p)$

$\begin{array}{lrrrr}C & 6.0 & -0.1577064373 & -0.2911845642 & -3.5432563557 \\ C & 6.0 & -0.1238987676 & -0.2585133169 & -2.0631907882 \\ C & 6.0 & -0.1215239358 & -0.2918118353 & 0.7044177610 \\ C & 6.0 & -0.6244782431 & -1.3429924278 & -1.3437527572\end{array}$

-663.1195003069 Hartree .182401 HARTREE/MOLECULE $-665.5185312616$

$-666.9900726504$ 


$\begin{array}{rrrrr}\mathrm{C} & 6.0 & 0.4137052739 & 0.8335022439 & -1.3738970913 \\ \mathrm{C} & 6.0 & 0.4121706940 & 0.8047268758 & 0.0044858436 \\ \mathrm{C} & 6.0 & -0.6533143386 & -1.4349126839 & 0.0673685263 \\ \mathrm{H} & 1.0 & -1.0137728558 & -2.1631879056 & -1.9247024960 \\ \mathrm{H} & 1.0 & 0.8291957292 & 1.6713081035 & -1.9024522319 \\ \mathrm{H} & 1.0 & 0.8532444731 & 1.6339583094 & 0.5343613924 \\ \mathrm{C} & 6.0 & -0.1087211139 & -0.2402951718 & 2.2033282681 \\ \mathrm{C} & 6.0 & -0.0511509141 & -0.1955208644 & 5.0257156565 \\ \mathrm{C} & 6.0 & 0.0227894119 & -1.4183567590 & 2.9478250363 \\ \mathrm{C} & 6.0 & -0.2304217938 & 0.9541070970 & 2.9172593093 \\ \mathrm{C} & 6.0 & -0.2013420008 & 0.9791470926 & 4.3068079921 \\ \mathrm{C} & 6.0 & 0.0573120552 & -1.3963017536 & 4.3316570291 \\ \mathrm{H} & 1.0 & 0.0831946228 & -2.3450836949 & 2.4110599611 \\ \mathrm{H} & 1.0 & -0.3744908341 & 1.8779091647 & 2.3861021002 \\ \mathrm{H} & 1.0 & -0.3052083075 & 1.9190363930 & 4.8238338169 \\ \mathrm{H} & 1.0 & 0.1673068348 & -2.3207581467 & 4.8744944407 \\ \mathrm{H} & 1.0 & -0.0266942850 & -0.1801047533 & 6.1024839547 \\ \mathrm{O} & 8.0 & -0.5727061683 & -1.1861320390 & -4.2229485080 \\ \mathrm{O} & 8.0 & 0.3376613084 & 0.8189100185 & -4.1142721602 \\ \mathrm{C} & 6.0 & 0.3377465870 & 0.8669344869 & -5.5183645552 \\ \mathrm{H} & 1.0 & 0.9343702380 & 0.0659408174 & -5.9346994751 \\ \mathrm{H} & 1.0 & 0.7668615064 & 1.8238670531 & -5.7824309203 \\ \mathrm{H} & 1.0 & -0.6683793133 & 0.7915252817 & -5.9092839723\end{array}$

Energy

ZPE

$M P 2 / 6-311+G(d, p)$

-686.2775067761 Hartree .225330 HARTREE/MOLECULE $B 3 L Y P / 6-311+G(d, p)$

$-688.7748585834$

$-690.3305591927$

2'-carbanion of 4-carbomethoxybiphenyl

$\mathrm{HF} / 6-31+\mathrm{G}(d, \mathrm{p})$

$\begin{array}{rrrrr}\text { C } & 6.0 & -0.1183198009 & -0.2947069393 & -3.4810901031 \\ \text { C } & 6.0 & -0.0854320501 & -0.2533662690 & -2.0077952525 \\ \text { C } & 6.0 & -0.0583626138 & -0.2551167106 & 0.8231013331 \\ \text { C } & 6.0 & -0.5487553821 & -1.3564191767 & -1.2842089875 \\ \text { C } & 6.0 & 0.4015060257 & 0.8426062536 & -1.3005599817 \\ \text { C } & 6.0 & 0.4141674972 & 0.8381348959 & 0.0825419934 \\ \text { C } & 6.0 & -0.5308939313 & -1.3590010018 & 0.0935560793 \\ \text { H } & 1.0 & -0.9221177466 & -2.2101067145 & -1.8199855303 \\ \text { H } & 1.0 & 0.7811879582 & 1.6956649129 & -1.8314693444 \\ \text { H } & 1.0 & 0.8251872557 & 1.6888763916 & 0.5939890522 \\ \text { H } & 1.0 & -0.8670945634 & -2.2142952381 & 0.6453760126 \\ \text { C } & 6.0 & -0.0592246181 & -0.2775164676 & 2.3160192973 \\ \text { C } & 6.0 & -0.0121283981 & -0.2134356742 & 5.0794127506 \\ \text { C } & 6.0 & 0.0481587968 & -1.5279029121 & 2.9746309073\end{array}$




$\begin{array}{rrrrr}\mathrm{C} & 6.0 & -0.1679032225 & 0.9444838289 & 2.9991932029 \\ \mathrm{C} & 6.0 & -0.1465151787 & 0.9862488056 & 4.3817626793 \\ \mathrm{C} & 6.0 & 0.0754655933 & -1.4112311490 & 4.3857047557 \\ \mathrm{H} & 1.0 & -0.2966086052 & 1.8698202551 & 2.4596290737 \\ \mathrm{H} & 1.0 & -0.2419531016 & 1.9248525602 & 4.9041774094 \\ \mathrm{H} & 1.0 & 0.1650814461 & -2.3140320959 & 4.9767834322 \\ \mathrm{H} & 1.0 & 0.0119804150 & -0.2036927947 & 6.1601458212 \\ \mathrm{O} & 8.0 & -0.5242376520 & -1.2088303881 & -4.1410731739 \\ \mathrm{O} & 8.0 & 0.3591965002 & 0.8136362282 & -4.0565563873 \\ \mathrm{C} & 6.0 & 0.3664540255 & 0.8516468093 & -5.4643249758 \\ \mathrm{H} & 1.0 & 0.9754250706 & 0.0540337955 & -5.8677047095 \\ \mathrm{H} & 1.0 & 0.7858070282 & 1.8111025246 & -5.7312231284 \\ \mathrm{H} & 1.0 & -0.6371441511 & 0.7622167673 & -5.8573794091\end{array}$

Energy

ZPE

$M P 2 / 6-311+G(d, p)$

$B 3 L Y P / 6-311+G(d, p)$

2-carbanion of 4-methylbiphenyl

$H F / 6-31+G(d, p)$

$\begin{array}{lrrrr}\mathrm{C} & 6.0 & -.1063369181 & .0822295899 & -.1042357837 \\ \mathrm{C} & 6.0 & -.0693746807 & .0695476248 & 1.3929007430 \\ \mathrm{C} & 6.0 & -.0555124976 & .0305731833 & 4.2219199006 \\ \mathrm{C} & 6.0 & -1.2423842162 & .2676149955 & 2.1337031139 \\ \mathrm{C} & 6.0 & 1.1091743491 & -.1282876862 & 2.1187538426 \\ \mathrm{C} & 6.0 & 1.1181715045 & -.1511875214 & 3.5087354638 \\ \mathrm{C} & 6.0 & -1.2366313696 & .2445892408 & 3.5175073284 \\ \mathrm{H} & 1.0 & -2.1495354847 & .4525384903 & 1.5917334776 \\ \mathrm{H} & 1.0 & 2.0412076943 & -.2412525845 & 1.5941830677 \\ \mathrm{H} & 1.0 & 2.0491086121 & -.03006008041 & 4.0314909048 \\ \mathrm{H} & 1.0 & -2.1594684818 & .3949675861 & 4.0539286486 \\ \mathrm{H} & 1.0 & -.0525288370 & .0150296502 & 5.2991051086 \\ \mathrm{C} & 6.0 & -.0637874786 & .0581094570 & -2.8933035572 \\ \mathrm{C} & 6.0 & .8075136868 & -.7210656874 & -.7986017933 \\ \mathrm{C} & 6.0 & -1.0637653929 & .8904207657 & -.7637827918 \\ \mathrm{C} & 6.0 & -.9719876951 & .8278342174 & -2.1746367307 \\ \mathrm{C} & 6.0 & .8359726006 & -.7379606430 & -2.1827134338 \\ \mathrm{H} & 1.0 & 1.4917742541 & -1.3642260437 & -.2659792071 \\ \mathrm{H} & 1.0 & -1.6659137522 & 1.4236343143 & -2.7567849127 \\ \mathrm{H} & 1.0 & 1.5399508810 & -1.3711261525 & -2.7024179080 \\ \mathrm{C} & 6.0 & -.0432375868 & .0636403487 & -4.4076935291 \\ \mathrm{H} & 1.0 & .9002435625 & .4455763908 & -4.7949033053 \\ \mathrm{H} & 1.0 & -.8386309174 & .6873897045 & -4.8047514798 \\ \mathrm{H} & 1.0 & -.1740218362 & -.9379884915 & -4.8141569012 \\ & & & & \end{array}$

-686.2772911244 Hartree .225243 HARTREE/MOLECULE $-688.7717373018$ $-690.3300872560$ 
Energy

ZPE

$M P 2 / 6-311+G(d, p)$

$B 3 L Y P / 6-311+G(d, p)$

2'-carbanion of 4-methylbiphenyl

$H F / 6-31+G(d, p)$

$\begin{array}{lrrrr}\mathrm{C} & 6.0 & .0076116966 & .0977577973 & .0185206365 \\ \mathrm{C} & 6.0 & .0316908323 & .1244415772 & 1.5172765226 \\ \mathrm{C} & 6.0 & .0114902639 & .0745633767 & 4.2840304805 \\ \mathrm{C} & 6.0 & -.3114541041 & -1.0480456167 & 2.2032018634 \\ \mathrm{C} & 6.0 & .4100499770 & 1.3235619331 & 2.1739886139 \\ \mathrm{C} & 6.0 & .3690045675 & 1.2207106374 & 3.5835943096 \\ \mathrm{C} & 6.0 & -.3270237906 & -1.0826396351 & 3.5893941431 \\ \mathrm{H} & 1.0 & -.5465291750 & -1.9526439193 & 1.6629494136 \\ \mathrm{H} & 1.0 & .6360334671 & 2.0894781050 & 4.1732981115 \\ \mathrm{H} & 1.0 & -.5846106575 & -1.9904284897 & 4.1122542198 \\ \mathrm{H} & 1.0 & .0047880452 & .0730726280 & 5.3653406385 \\ \mathrm{C} & 6.0 & .0280541961 & .0663242473 & -2.8307723076 \\ \mathrm{C} & 6.0 & .8943361654 & .8930162759 & -.7216603693 \\ \mathrm{C} & 6.0 & -.8748376763 & -.6921614561 & -.7153256835 \\ \mathrm{C} & 6.0 & -.8624013253 & -.7105333691 & -2.1086429962 \\ \mathrm{C} & 6.0 & .9057168456 & .8728849485 & -2.1031427571 \\ \mathrm{H} & 1.0 & 1.5627684954 & 1.5330217490 & -.1789389651 \\ \mathrm{H} & 1.0 & -1.6049989164 & -1.2918819736 & -.2012884989 \\ \mathrm{H} & 1.0 & -1.5692408547 & -1.3370443255 & -2.6300938259 \\ \mathrm{H} & 1.0 & 1.6109283553 & 1.4970835319 & -2.6306783125 \\ \mathrm{C} & 6.0 & .0480483749 & .0607917722 & -4.3431247229 \\ \mathrm{H} & 1.0 & 1.0346456570 & -.1868115952 & -4.7282084658 \\ \mathrm{H} & 1.0 & -.2219945519 & 1.0328378300 & -4.7501116401 \\ \mathrm{H} & 1.0 & -.6520757305 & -.6673560287 & -4.7418602505\end{array}$

Energy

ZPE

$M P 2 / 6-311+G(d, p)$

$B 3 L Y P / 6-311+G(d, p)$
-498.6526973189 Hartree .207478 HARTREE/MOLECULE -500.5827198937 Hartree

-501.7833436215 Hartree

2'-carbanion of 4-N,N-dimethylaminobiphenyl

$H F / 6-31+G(d, p)$
C
$6.0 \quad 0.0407120584$
0.7577982457
$-0.4965067689$
$\begin{array}{lll}C & 6.0 & -0.0710715347\end{array}$
$2.0565351819-1.2365951211$
C
$6.0-0.1576188284$
$4.4514274954-2.6193398249$ 


$\begin{array}{lrrrr}\mathrm{C} & 6.0 & 1.0539897996 & 2.8903699395 & -1.2817405385 \\ \mathrm{C} & 6.0 & -1.3056057454 & 2.3865621808 & -1.8519133119 \\ \mathrm{C} & 6.0 & -1.2681812132 & 3.6195155836 & -2.5432577028 \\ \mathrm{C} & 6.0 & 1.0213813100 & 4.0915883251 & -1.9733617075 \\ \mathrm{H} & 1.0 & 1.9589786889 & 2.6221454185 & -0.7578763079 \\ \mathrm{H} & 1.0 & -2.1633823886 & 3.9535943210 & -3.0537158245 \\ \mathrm{H} & 1.0 & 1.8877710675 & 4.7337038070 & -1.9978987737 \\ \mathrm{H} & 1.0 & -0.2072065877 & 5.3814631292 & -3.1686959605 \\ \mathrm{C} & 6.0 & 0.1936993454 & -1.7023428695 & 0.9120414458 \\ \mathrm{C} & 6.0 & -1.0773639526 & 0.2063094122 & 0.1429511782 \\ \mathrm{C} & 6.0 & 1.2280563027 & 0.0295303783 & -0.4132505141 \\ \mathrm{C} & 6.0 & 1.3048655535 & -1.1758236260 & 0.2783909459 \\ \mathrm{C} & 6.0 & -1.0024786337 & -0.9898062681 & 0.8334147900 \\ \mathrm{H} & 1.0 & -2.0080715547 & 0.7343606243 & 0.0671301706 \\ \mathrm{H} & 1.0 & 2.1073741186 & 0.3877261116 & -0.9183292884 \\ \mathrm{H} & 1.0 & 2.2322608895 & -1.7219888564 & 0.3160467913 \\ \mathrm{H} & 1.0 & -1.8884860252 & -1.3735638073 & 1.3125208337 \\ \mathrm{~N} & 7.0 & 0.3068042596 & -2.9584610929 & 1.6054954646 \\ \mathrm{C} & 6.0 & -0.4673936547 & -4.0272016117 & 1.0085630997 \\ \mathrm{C} & 6.0 & 0.0996373349 & -2.8634906282 & 3.0352451070 \\ \mathrm{H} & 1.0 & -0.9351643201 & -2.6409797283 & 3.3110197580 \\ \mathrm{H} & 1.0 & 0.7315915557 & -2.0848273300 & 3.4434944176 \\ \mathrm{H} & 1.0 & 0.3733600601 & -3.8059495217 & 3.5029184272 \\ \mathrm{H} & 1.0 & -1.5471706042 & -3.8990226213 & 1.1256694185 \\ \mathrm{H} & 1.0 & -0.1918372753 & -4.9732308967 & 1.4678716024 \\ \mathrm{H} & 1.0 & -0.2494439399 & -4.0859399219 & -0.0502940821 \\ & & & & \end{array}$

Energy

ZPE

$M P 2 / 6-311+G(d, p)$
-592.6949562409 Hartree

.256300 HARTREE/MOLECULE

-594.8826958823 Hartree

2-carbanion of 4-N,N-dimethylaminobiphenyl

$H F / 6-31+G(d, p)$

$\begin{array}{lrrrr}\mathrm{C} & 6.0 & .0178689151 & .7458215208 & -.3106767088 \\ \mathrm{C} & 6.0 & -.0404049517 & 2.0068408063 & -1.1159288577 \\ \mathrm{C} & 6.0 & -.1639084781 & 4.4185547860 & -2.5924067076 \\ \mathrm{C} & 6.0 & .5407235757 & 2.1235107101 & -2.3825278863 \\ \mathrm{C} & 6.0 & -.7040631853 & 3.1372254451 & -.6193294649 \\ \mathrm{C} & 6.0 & -.7618127734 & 4.3177141149 & -1.3396135990 \\ \mathrm{C} & 6.0 & .4841115454 & 3.3079412856 & -3.1082738977 \\ \mathrm{H} & 1.0 & 1.0239881624 & 1.2703212393 & -2.8243960026 \\ \mathrm{H} & 1.0 & -1.1815782069 & 3.0552767768 & .3377598115 \\ \mathrm{H} & 1.0 & -1.2779676443 & 5.1675249832 & -.9230823198 \\ \mathrm{H} & 1.0 & .9381904737 & 3.3536335263 & -4.0849323747 \\ \mathrm{H} & 1.0 & -.2119946125 & 5.3375009137 & -3.1528322419\end{array}$




$\begin{array}{lrrrr}\mathrm{C} & 6.0 & .1973827970 & -1.6487506966 & 1.1013216717 \\ \mathrm{C} & 6.0 & -1.0416264646 & .4457088147 & .5788082782 \\ \mathrm{C} & 6.0 & 1.1306653937 & -.0918399441 & -.4600783272 \\ \mathrm{C} & 6.0 & 1.2304810339 & -1.2815640193 & .2391791214 \\ \mathrm{C} & 6.0 & -.8847360699 & -.7893377301 & 1.2534648757 \\ \mathrm{H} & 1.0 & 1.9540052184 & .1925211801 & -1.0978418458 \\ \mathrm{H} & 1.0 & 2.1041020241 & -1.9062434619 & .1477810841 \\ \mathrm{H} & 1.0 & -1.6629314073 & -1.0858172699 & 1.9416156609 \\ \mathrm{~N} & 7.0 & .3193892012 & -2.9076007150 & 1.7899025536 \\ \mathrm{C} & 6.0 & -.0142912258 & -4.0505562237 & .9660476494 \\ \mathrm{C} & 6.0 & -.3101488050 & -2.9889052737 & 3.0865556371 \\ \mathrm{H} & 1.0 & -1.0837534191 & -4.1048843880 & .7377541898 \\ \mathrm{H} & 1.0 & .2695260352 & -4.9666601112 & 1.4799034320 \\ \mathrm{H} & 1.0 & .5251570396 & -4.0059615449 & .0293965189 \\ \mathrm{H} & 1.0 & -1.4003785936 & -3.0593101491 & 3.0442073782 \\ \mathrm{H} & 1.0 & -.0518369610 & -2.1185276489 & 3.6756562769 \\ \mathrm{H} & 1.0 & .0558296360 & -3.8741023727 & 3.6025529698\end{array}$

Energy

ZPE

$M P 2 / 6-311+G(d, p)$

2-carbanion of 4-methoxybiphenyl

$H F / 6-31+G(d, p)$

$\begin{array}{lrrrr}\mathrm{C} & 6.0 & -.0799082731 & -.0041936655 & .1411309792 \\ \mathrm{C} & 6.0 & -.1651548995 & .0278475992 & 1.6341684591 \\ \mathrm{C} & 6.0 & -.2814720875 & .0409774437 & 4.4631026280 \\ \mathrm{C} & 6.0 & .9631265776 & -.2571216396 & 2.4162643760 \\ \mathrm{C} & 6.0 & -1.3437344131 & .3388353434 & 2.3203327461 \\ \mathrm{C} & 6.0 & -1.4041715914 & .3419423355 & 3.7091839261 \\ \mathrm{C} & 6.0 & .9056491866 & -.2542048345 & 3.7988607141 \\ \mathrm{H} & 1.0 & 1.8832075413 & -.4679148156 & 1.9064136895 \\ \mathrm{H} & 1.0 & -2.2250102586 & .6072220874 & 1.7651359674 \\ \mathrm{H} & 1.0 & -2.3313359072 & .5923163503 & 4.1990959910 \\ \mathrm{H} & 1.0 & 1.7929829822 & -.4847832893 & 4.3660065275 \\ \mathrm{H} & 1.0 & -.3241658186 & .0435833337 & 5.5396182741 \\ \mathrm{C} & 6.0 & -.0281460439 & -.0768763748 & -2.6295351646 \\ \mathrm{C} & 6.0 & -1.2206129654 & -.3616082140 & -.5872228478 \\ \mathrm{C} & 6.0 & 1.1489751724 & .3112981091 & -.4890368467 \\ \mathrm{C} & 6.0 & 1.1039138050 & .2630815379 & -1.9034372883 \\ \mathrm{C} & 6.0 & -1.2101256470 & -.4017825868 & -1.9698924844 \\ \mathrm{H} & 1.0 & -2.1325177916 & -.6415685698 & -.0820941501 \\ \mathrm{H} & 1.0 & 2.0061005434 & .5022676871 & -2.4441210541 \\ \mathrm{H} & 1.0 & -2.0791155456 & -.6892787694 & -2.5367188485 \\ \mathrm{O} & 8.0 & -.0890844518 & -.1347875269 & -4.0001944706\end{array}$




$\begin{array}{lrrrr}\mathrm{C} & 6.0 & 1.0560573875 & .1267511261 & -4.7423041597 \\ \mathrm{H} & 1.0 & 1.8605135375 & -.5610159700 & -4.4977693717 \\ \mathrm{H} & 1.0 & 1.4099346815 & 1.1431064523 & -4.5938944727 \\ \mathrm{H} & 1.0 & .7835528263 & -.0035309024 & -5.7830142718 \\ & & & \\ \text { Energy } & & & -573.5017480223 \text { Hartree } \\ \text { ZPE } & & .213691 \text { HARTREE/MOLECULE } \\ \text { MP2/6-311+G(d,p) } & & -575.6465054722 \text { Hartree } \\ \text { B3LYP/6-311+G(d,p) } & & -576.9849017274 \text { Hartree }\end{array}$

2'-carbanion of 4-methoxybiphenyl

$H F / 6-31+G(d, p)$

$\begin{array}{lrrrr}\mathrm{C} & 6.0 & .0213868346 & .2852071533 & -.0884270135 \\ \mathrm{C} & 6.0 & -.3525364857 & 1.3636848207 & -1.0616882619 \\ \mathrm{C} & 6.0 & -.9275320344 & 3.3834209992 & -2.8638632842 \\ \mathrm{C} & 6.0 & .6727330458 & 1.9713713161 & -1.7966423786 \\ \mathrm{C} & 6.0 & -1.7206183872 & 1.7101978029 & -1.2070107680 \\ \mathrm{C} & 6.0 & -1.9322172350 & 2.7512744314 & -2.1389557975 \\ \mathrm{C} & 6.0 & .3951843593 & 2.9864477918 & -2.7010413240 \\ \mathrm{H} & 1.0 & 1.6957922006 & 1.6428526422 & -1.6893328517 \\ \mathrm{H} & 1.0 & -2.9460064919 & 3.0915035068 & -2.3128322383 \\ \mathrm{H} & 1.0 & 1.1882027345 & 3.4452666057 & -3.2706173671 \\ \mathrm{H} & 1.0 & -1.1694038590 & 4.1740963274 & -3.5607506152 \\ \mathrm{C} & 6.0 & .6874710999 & -1.7850591763 & 1.7231223209 \\ \mathrm{C} & 6.0 & 1.2246101007 & .2842322298 & .6250474451 \\ \mathrm{C} & 6.0 & -.8391710765 & -.7815075673 & .1579263453 \\ \mathrm{C} & 6.0 & -.5205941787 & -1.8071054406 & 1.0419030124 \\ \mathrm{C} & 6.0 & 1.5587089588 & -.7271637154 & 1.5117344404 \\ \mathrm{H} & 1.0 & 1.9114279082 & 1.1033303371 & .5059679976 \\ \mathrm{H} & 1.0 & -1.7837035837 & -.7913253804 & -.3508748510 \\ \mathrm{H} & 1.0 & -1.2256539825 & -2.6055252845 & 1.1837890341 \\ \mathrm{H} & 1.0 & 2.4870970552 & -.7002506563 & 2.0554593528 \\ \mathrm{O} & 8.0 & 1.0932136883 & -2.7402971002 & 2.6138078654 \\ \mathrm{C} & 6.0 & .2730093022 & -3.8389311213 & 2.8489158835 \\ \mathrm{H} & 1.0 & -.6883033815 & -3.5424515625 & 3.2590762378 \\ \mathrm{H} & 1.0 & .7883928917 & -4.4570317858 & 3.5722033914 \\ \mathrm{H} & 1.0 & .1076182943 & -4.4162154028 & 1.9431272253 \\ & & & & \\ \mathrm{Energy} & & & -573.4969209563 \mathrm{Hartree} \\ \mathrm{ZPE} & & & .213560 \mathrm{HARTREE} / \mathrm{MOLECULE} \\ \mathrm{MP} 2 / 6-311+\mathrm{G}(\mathrm{d}, \mathrm{p}) & & -575.6438026917 \mathrm{Hartree} \\ \mathrm{B} 3 \mathrm{LYP} / 6-311+\mathrm{G}(\mathrm{d}, \mathrm{p}) & & -576.9814497925 \mathrm{Hartree} \\ & & & & \end{array}$


2'-carbanion of 3-phenylfuran

$H F / 6-31+G(d, p)$

$\begin{array}{lrrrr}\mathrm{C} & 6.0 & 0.0158217274 & 0.1737512269 & -1.2228674365 \\ \mathrm{C} & 6.0 & 0.0148402356 & -0.2817281743 & 0.1936669993 \\ \mathrm{C} & 6.0 & 0.0003403249 & -1.0386613434 & 2.8516954665 \\ \mathrm{C} & 6.0 & 0.0360513585 & -1.6791791649 & 0.4514400721 \\ \mathrm{C} & 6.0 & -0.0054731373 & 0.6845826111 & 1.2017289990 \\ \mathrm{C} & 6.0 & -0.0148094353 & 0.3145102430 & 2.5413116187 \\ \mathrm{C} & 6.0 & 0.0279404425 & -1.9844138781 & 1.8282739212 \\ \mathrm{H} & 1.0 & -0.0114187495 & 1.7370081068 & 0.9596334250 \\ \mathrm{H} & 1.0 & -0.0320941688 & 1.0641227562 & 3.3168749803 \\ \mathrm{H} & 1.0 & 0.0423161803 & -3.0253031996 & 2.1289297100 \\ \mathrm{H} & 1.0 & -0.0082237539 & -1.3522584872 & 3.8865332817 \\ \mathrm{C} & 6.0 & 0.1261014607 & -0.5992970729 & -2.3197146772 \\ \mathrm{C} & 6.0 & -0.1076831895 & 1.5214344534 & -1.7514782230 \\ \mathrm{H} & 1.0 & 0.2319467353 & -1.6534655265 & -2.4335048071 \\ \mathrm{O} & 8.0 & 0.0827521734 & 0.1390952096 & -3.4622942968 \\ \mathrm{C} & 6.0 & -0.0601390556 & 1.4182547103 & -3.0881844452 \\ \mathrm{H} & 1.0 & -0.2224508473 & 2.4332610101 & -1.2012936998 \\ \mathrm{H} & 1.0 & -0.1171978126 & 2.1414840327 & -3.8746446081\end{array}$

Energy

ZPE

$M P 2 / 6-311+G(d, p)$

$B 3 L Y P / 6-311+G(d, p)$

-457.5407665136 Hartree 0.146540 HARTREE/MOLECULE -459.2298829273 Hartree

-460.2831012574 Hartree

2-carbanion of 3-phenylfuran

$H F / 6-31+G(d, p)$

$\begin{array}{lrrrr}\mathrm{C} & 6.0 & .0180454368 & .1501180132 & -1.3755640486 \\ \mathrm{C} & 6.0 & .0288323267 & .0238565648 & .0975695715 \\ \mathrm{C} & 6.0 & .0333727037 & -.1384526451 & 2.9168183394 \\ \mathrm{C} & 6.0 & -.1490833192 & -1.1964306623 & .7602040829 \\ \mathrm{C} & 6.0 & .2251414032 & 1.1593626973 & .8907205183 \\ \mathrm{C} & 6.0 & .2266466241 & 1.0785982054 & 2.2746426230 \\ \mathrm{C} & 6.0 & -.1538799246 & -1.2769623131 & 2.1445207778 \\ \mathrm{H} & 1.0 & -.2767150465 & -2.0982285980 & .1871851601 \\ \mathrm{H} & 1.0 & .3806805703 & 2.0962348020 & .3905100251 \\ \mathrm{H} & 1.0 & .3801811564 & 1.9726688401 & 2.8567398274 \\ \mathrm{H} & 1.0 & -.2955594770 & -2.2337466534 & 2.6204128592 \\ \mathrm{H} & 1.0 & .0351282499 & -.1997696363 & 3.9922356549 \\ \mathrm{C} & 6.0 & -.4036431586 & -.7974317199 & -2.2431067218 \\ \mathrm{C} & 6.0 & .4507372544 & 1.3391544094 & -2.1363341495 \\ \mathrm{H} & 1.0 & -.8103609232 & -1.7817878567 & -2.1138827958\end{array}$




$\begin{array}{lrrrr}\mathrm{C} & 6.0 & .2255799600 & .9160545583 & -3.3972983354 \\ \mathrm{H} & 1.0 & .3652292922 & 1.3717830490 & -4.3582322020 \\ 0 & 8.0 & -.2942099884 & -.3766751848 & -3.5020306164 \\ & & & \\ \text { Energy } & & -457.5622506807 \text { Hartree } \\ \text { ZPE } & & .146515 \text { HARTREE/MOLECULE } \\ \text { MP2/6-311+G(d,p) } & -459.2484225596 \text { Hartree } \\ \text { B3LYP/6-311+G(d,p) } & -460.3052002960 \text { Hartree }\end{array}$

4'-carbanion of 3-phenylfuran

$H F / 6-31+G(d, p)$

$\begin{array}{lrrrr}\mathrm{C} & 6.0 & 0.0158217274 & 0.1737512269 & -1.2228674365 \\ \mathrm{C} & 6.0 & 0.0148402356 & -0.2817281743 & 0.1936669993 \\ \mathrm{C} & 6.0 & 0.0003403249 & -1.0386613434 & 2.8516954665 \\ \mathrm{C} & 6.0 & 0.0360513585 & -1.6791791649 & 0.4514400721 \\ \mathrm{C} & 6.0 & -0.0054731373 & 0.6845826111 & 1.2017289990 \\ \mathrm{C} & 6.0 & -0.0148094353 & 0.3145102430 & 2.5413116187 \\ \mathrm{C} & 6.0 & 0.0279404425 & -1.9844138781 & 1.8282739212 \\ \mathrm{H} & 1.0 & -0.0114187495 & 1.7370081068 & 0.9596334250 \\ \mathrm{H} & 1.0 & -0.0320941688 & 1.0641227562 & 3.3168749803 \\ \mathrm{H} & 1.0 & 0.0423161803 & -3.0253031996 & 2.1289297100 \\ \mathrm{H} & 1.0 & -0.0082237539 & -1.3522584872 & 3.8865332817 \\ \mathrm{C} & 6.0 & 0.1261014607 & -0.5992970729 & -2.3197146772 \\ \mathrm{C} & 6.0 & -0.1076831895 & 1.5214344534 & -1.7514782230 \\ \mathrm{H} & 1.0 & 0.2319467353 & -1.6534655265 & -2.4335048071 \\ 0 & 8.0 & 0.0827521734 & 0.1390952096 & -3.4622942968 \\ \mathrm{C} & 6.0 & -0.0601390556 & 1.4182547103 & -3.0881844452 \\ \mathrm{H} & 1.0 & -0.2224508473 & 2.4332610101 & -1.2012936998 \\ \mathrm{H} & 1.0 & -0.1171978126 & 2.1414840327 & -3.8746446081 \\ & & & & \\ \text { Energy } & & & -457.5497576335 \\ \text { ZPE } & & & .146972 \text { HARTREE/MOLECULE } \\ \text { MP2/6-311+G(d,p) } & & -459.2421124387 \text { Hartree } \\ \text { B3LYP/6-311+G(d,p) } & & -460.2957340247 \text { Hartree }\end{array}$




\section{NMR Spectra as Proof of Purity}

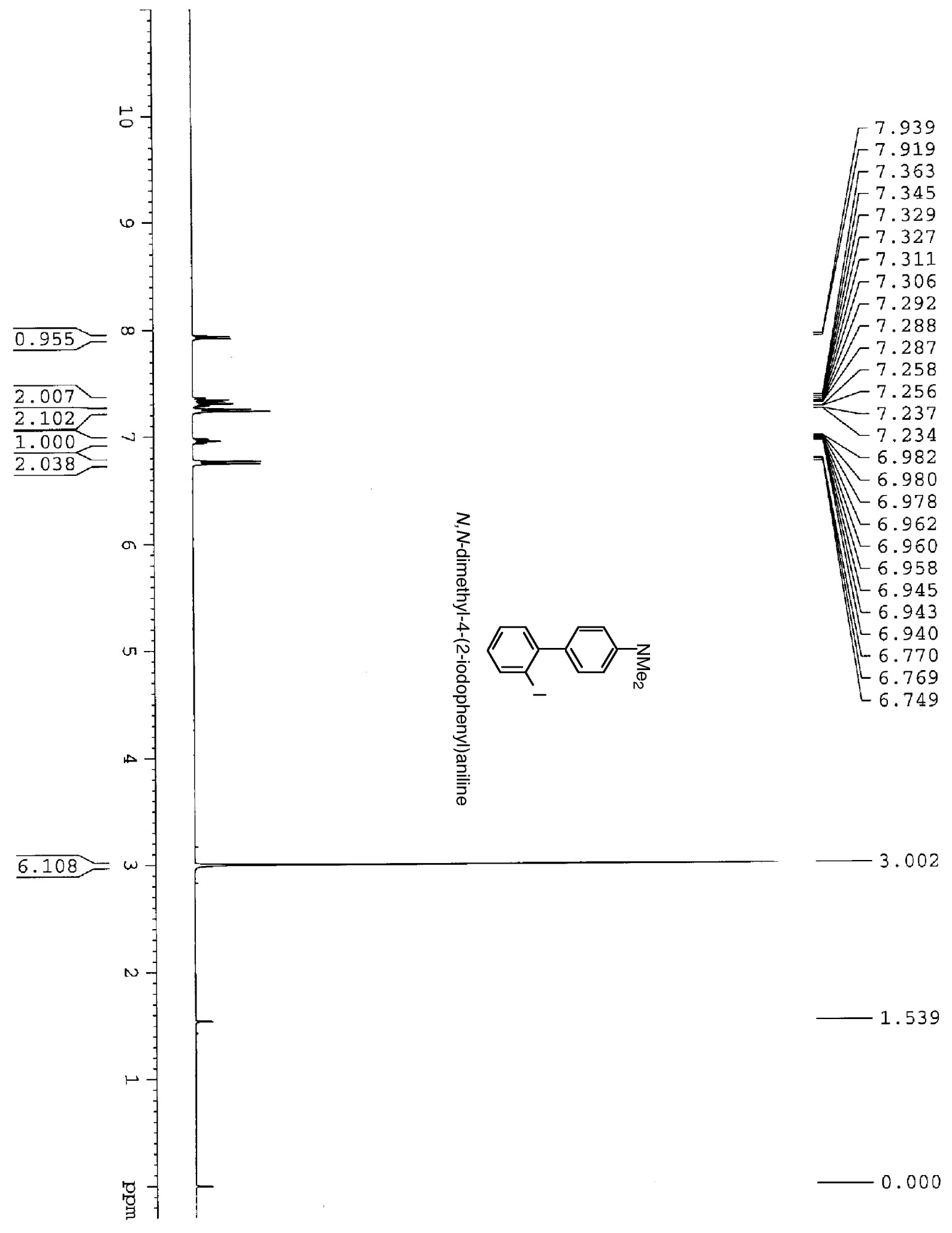



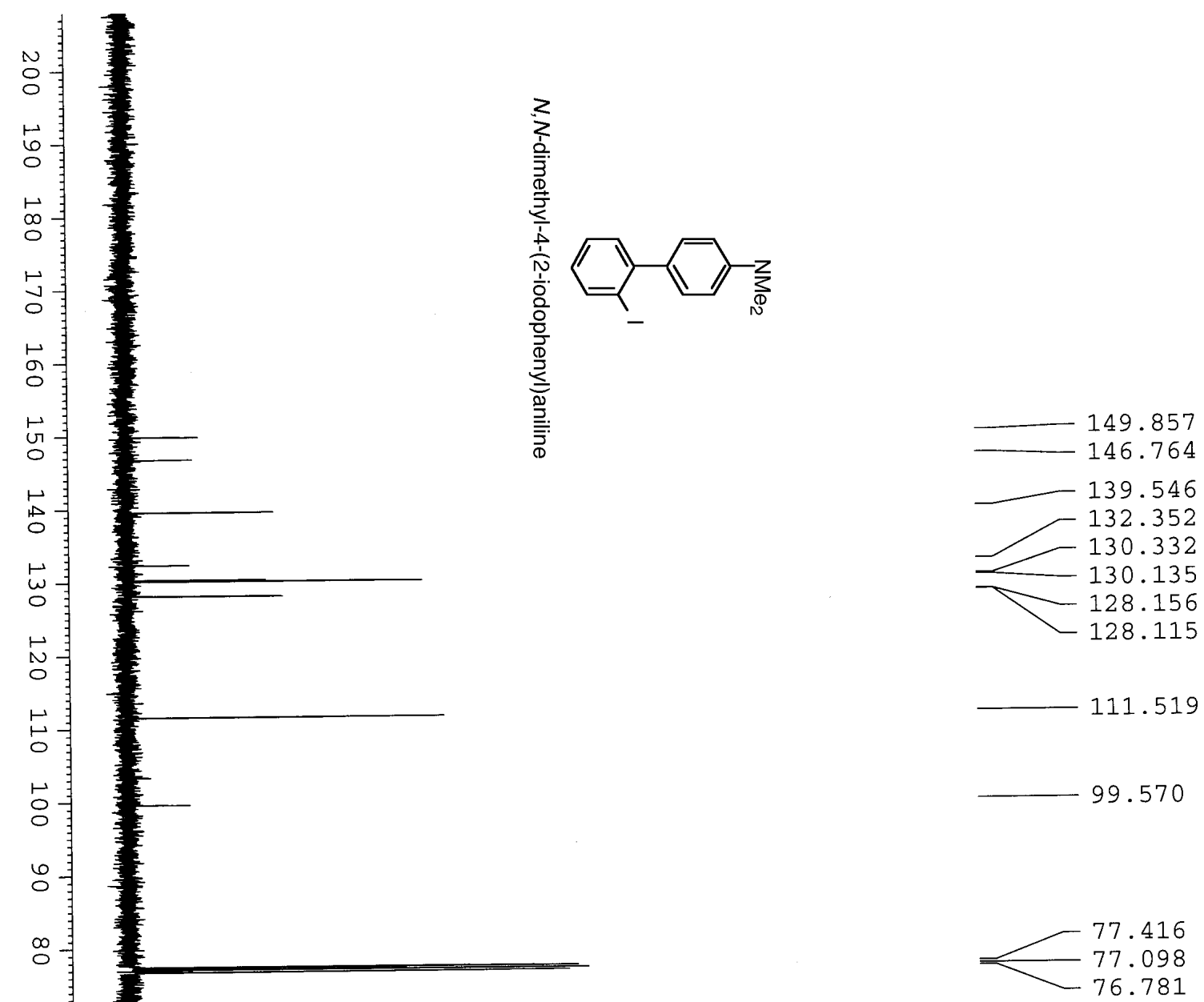

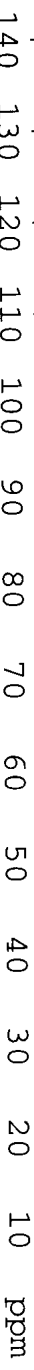

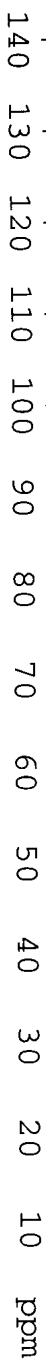

v

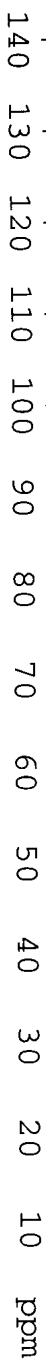

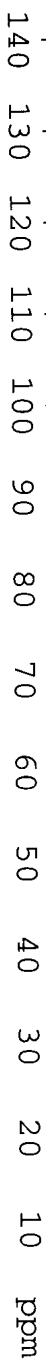

N

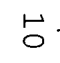

음
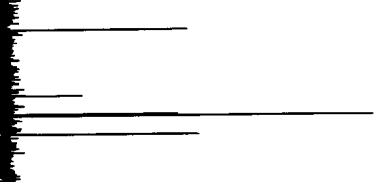

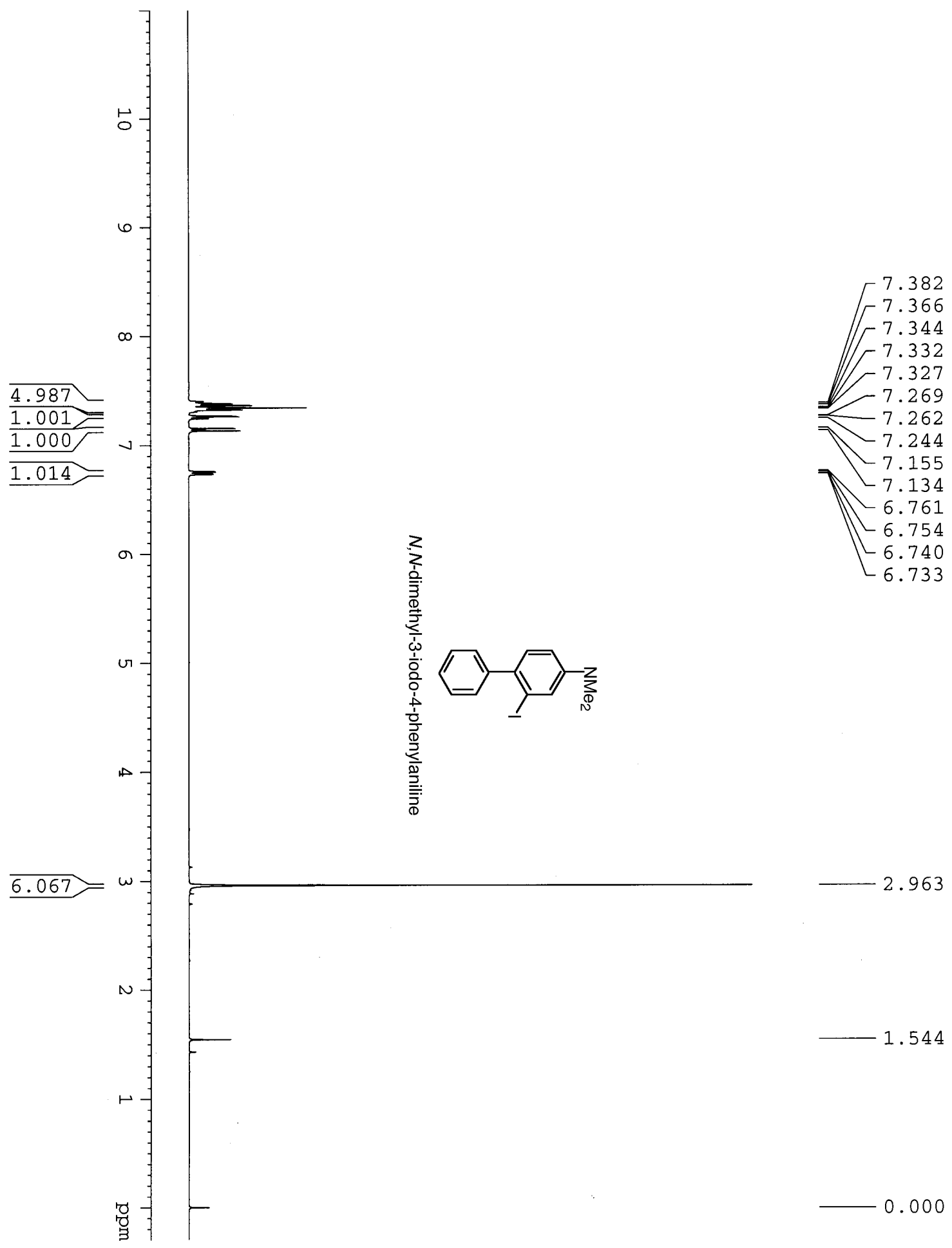

0.000 

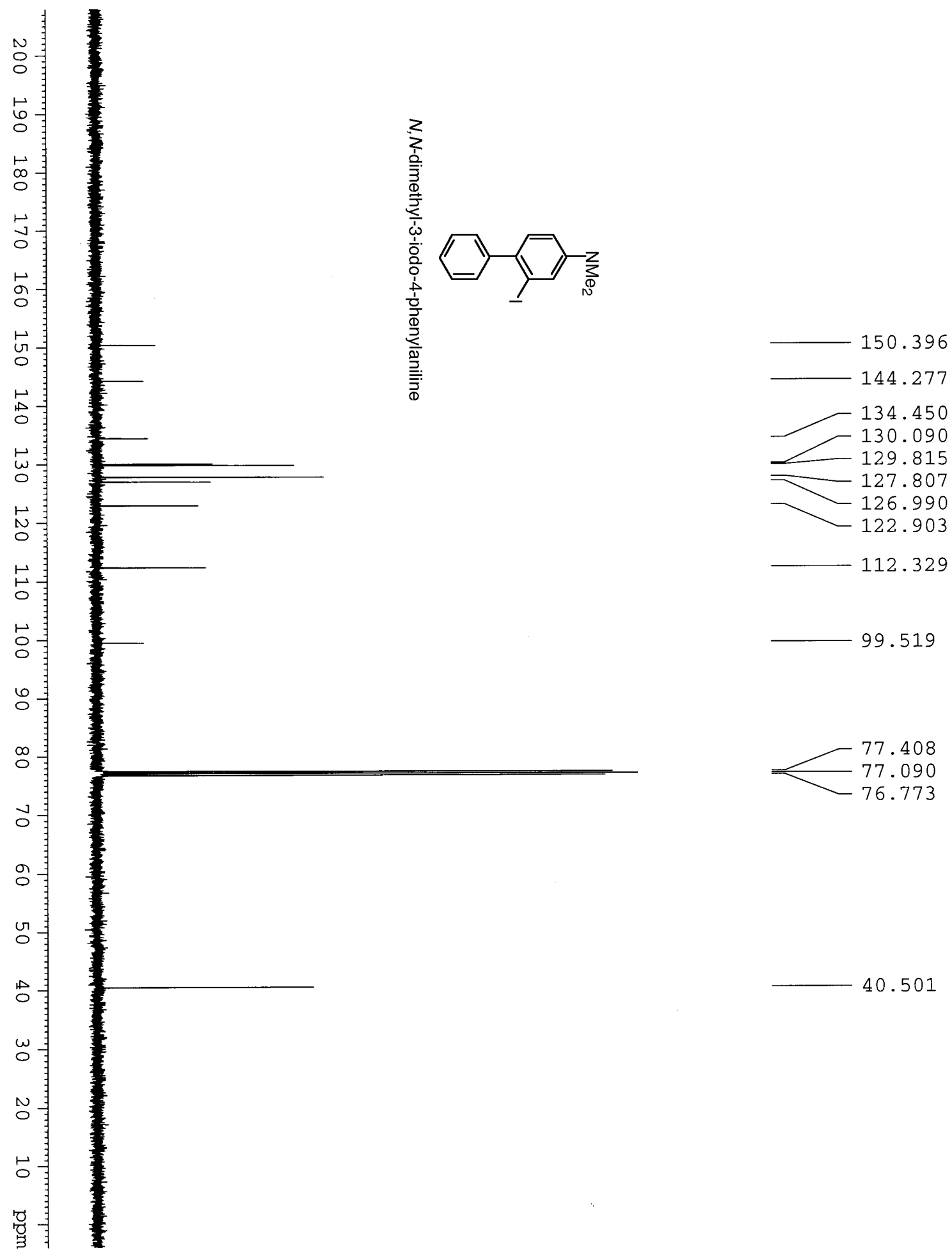

40.501 

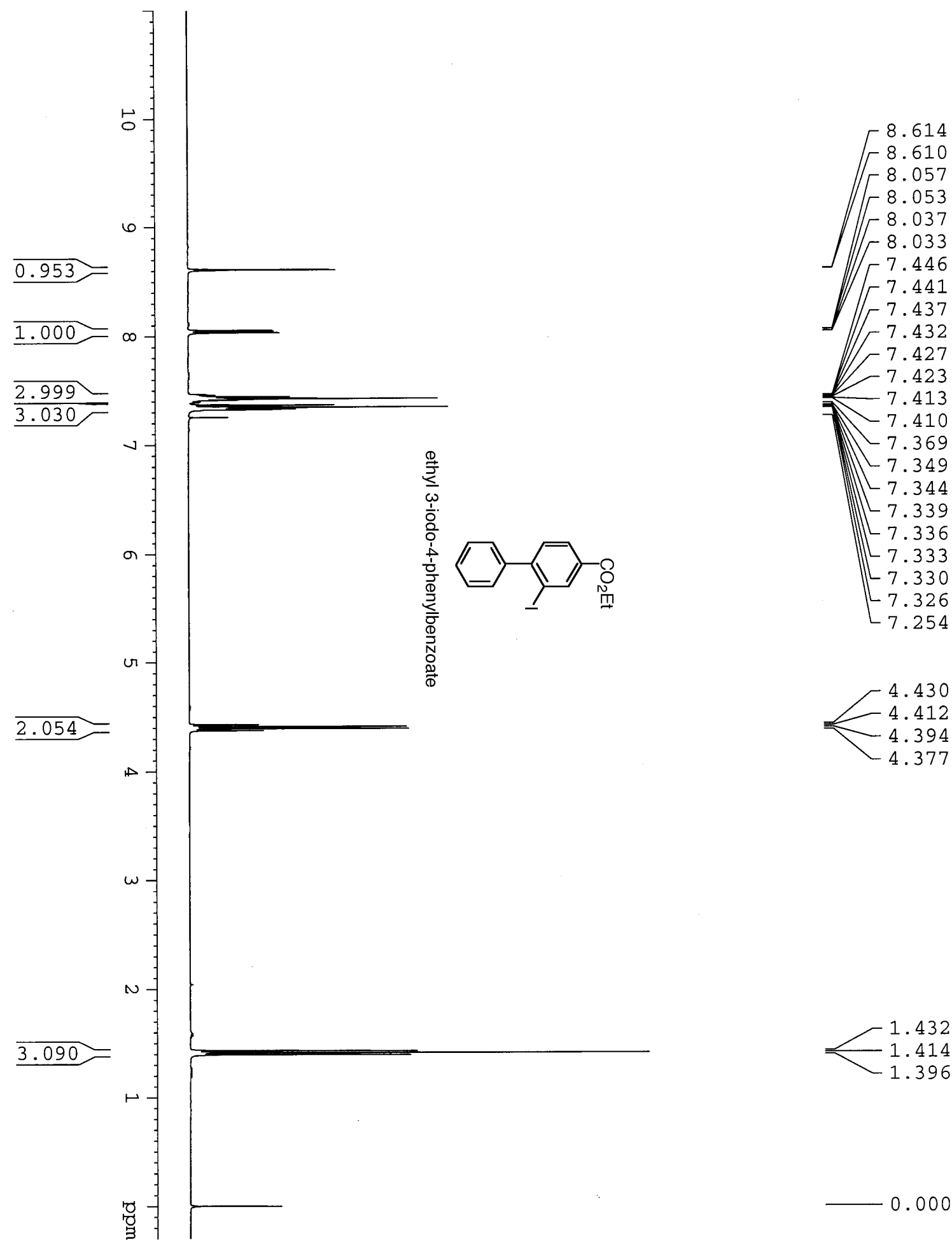

1. 432

1. 414

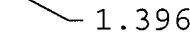

0.000 


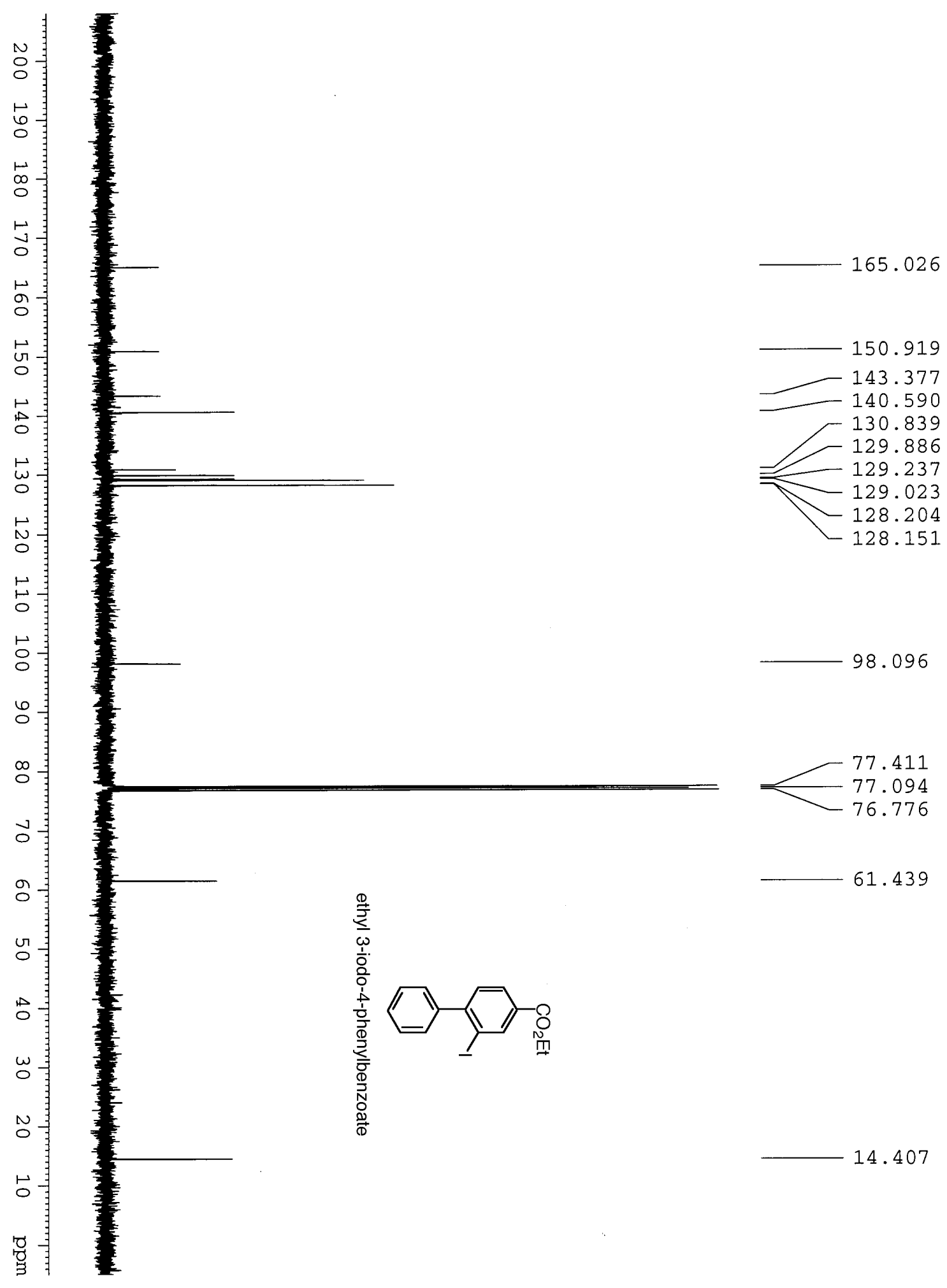




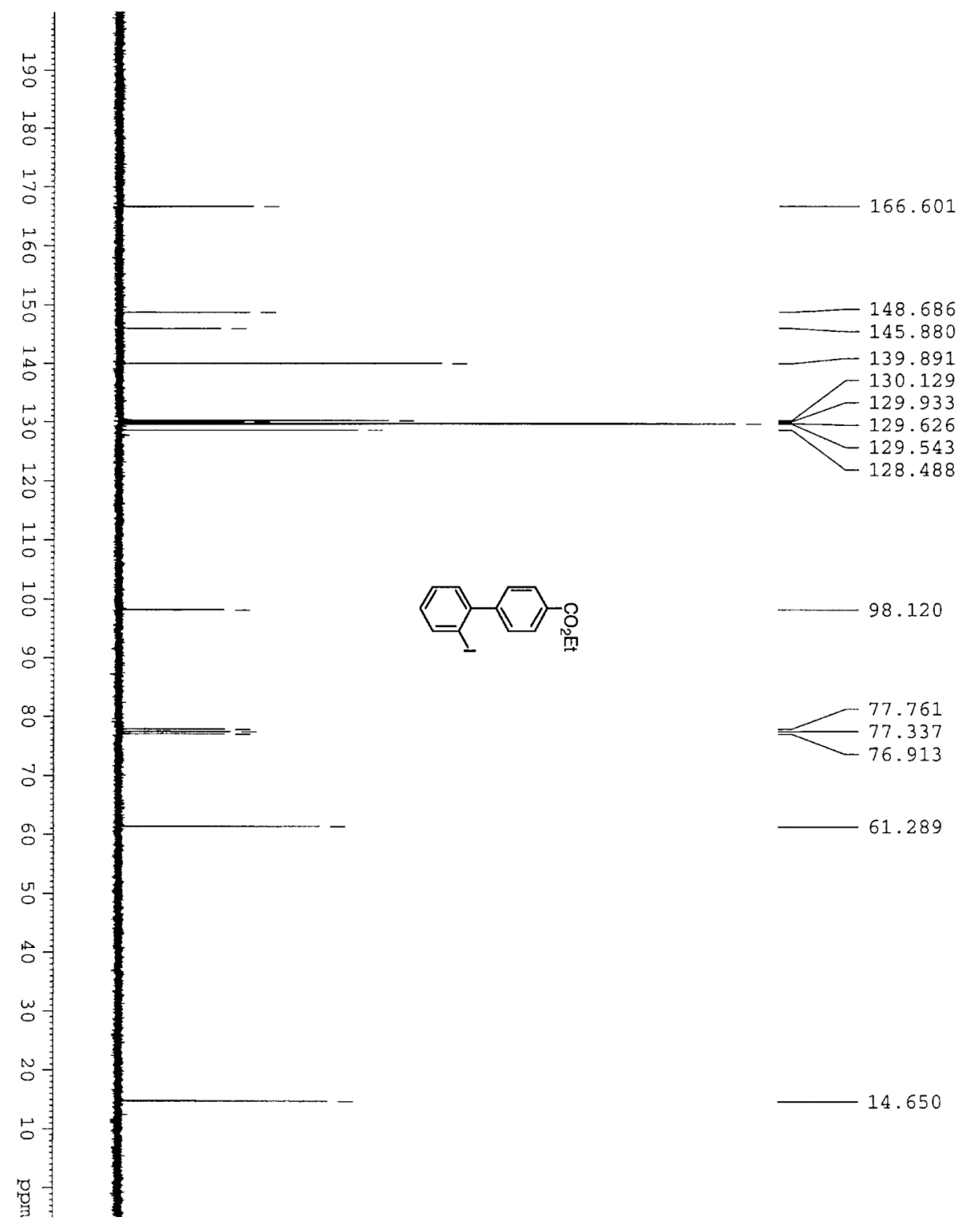



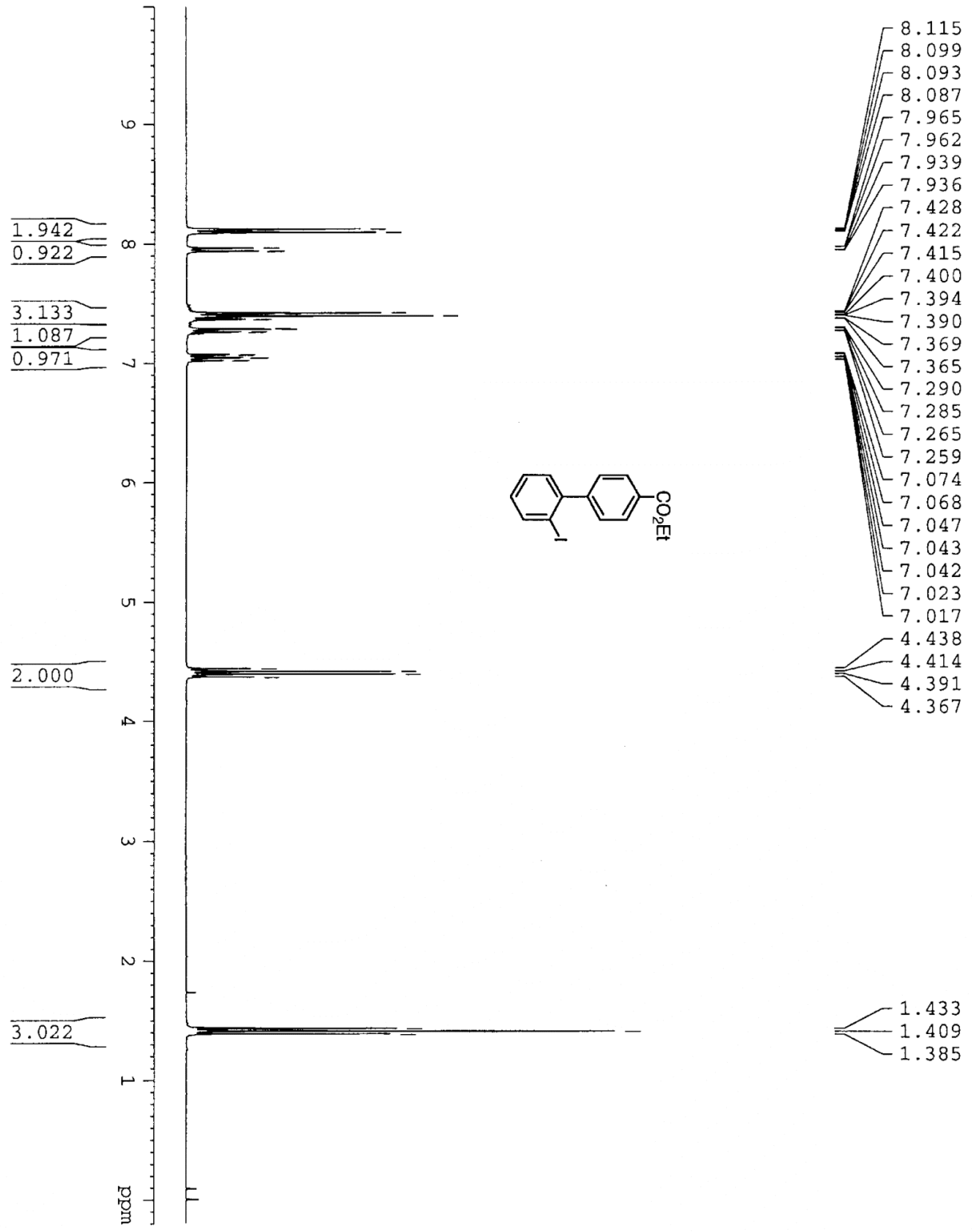

$-1.433$

1.409
-1.385 

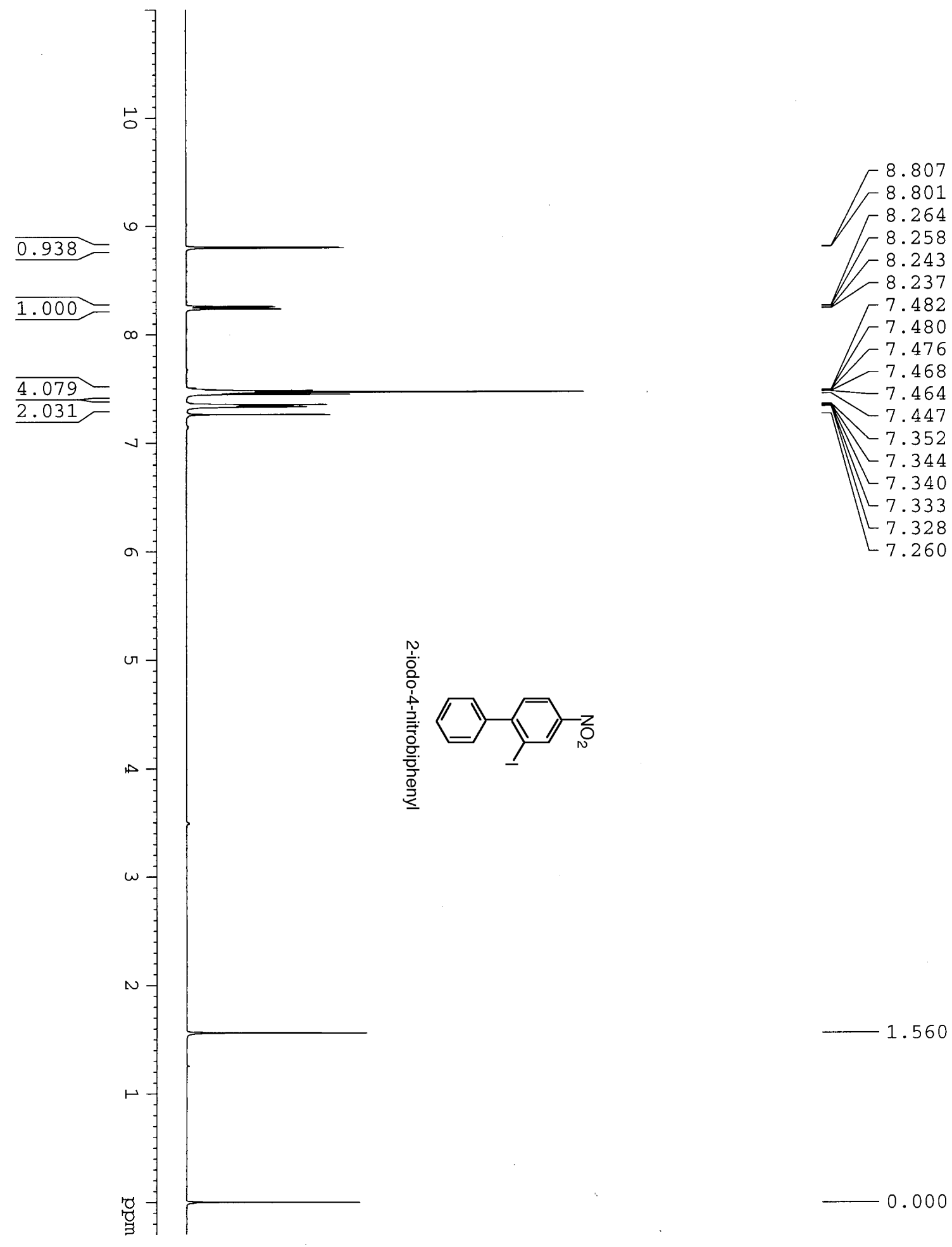

$-0.000$ 


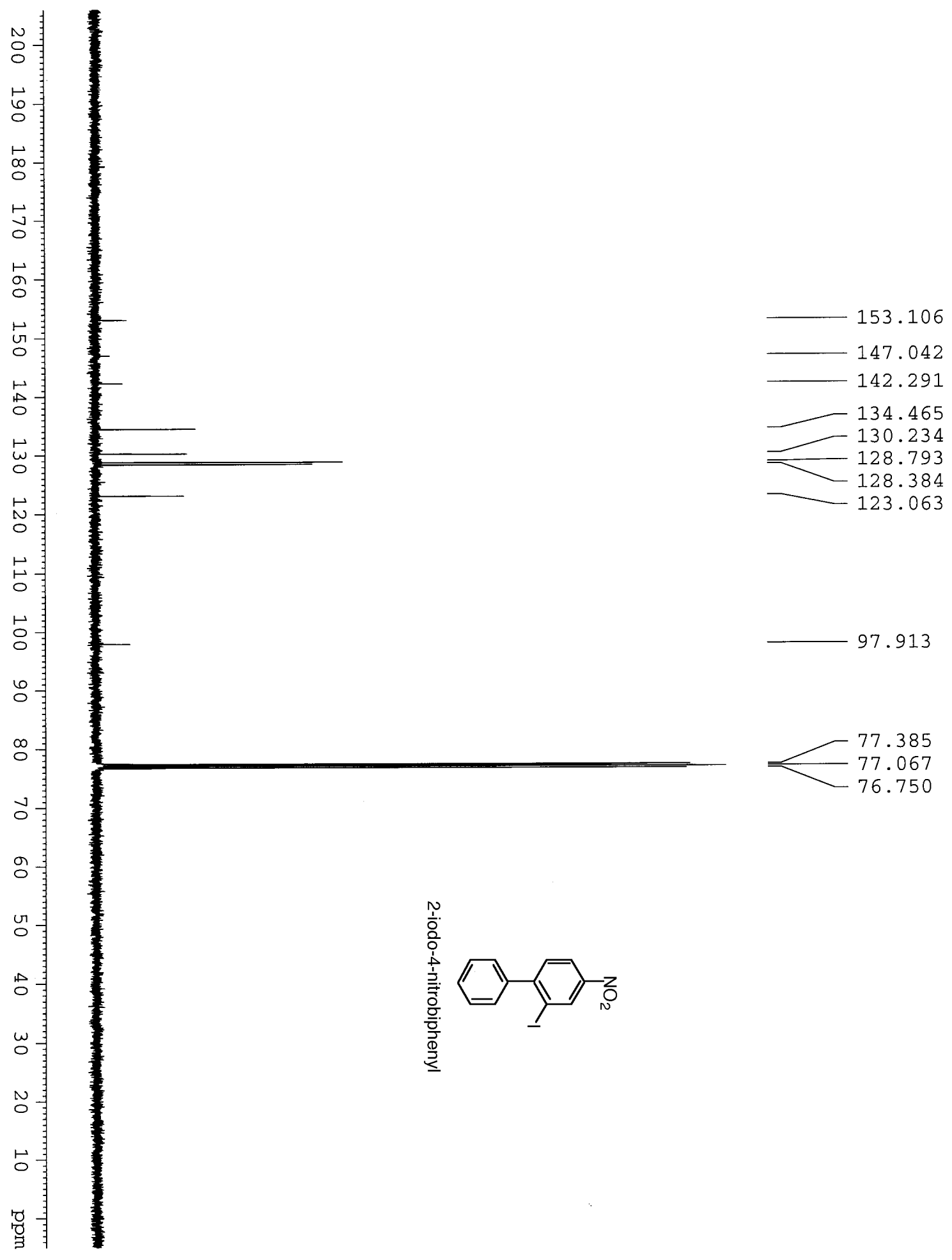



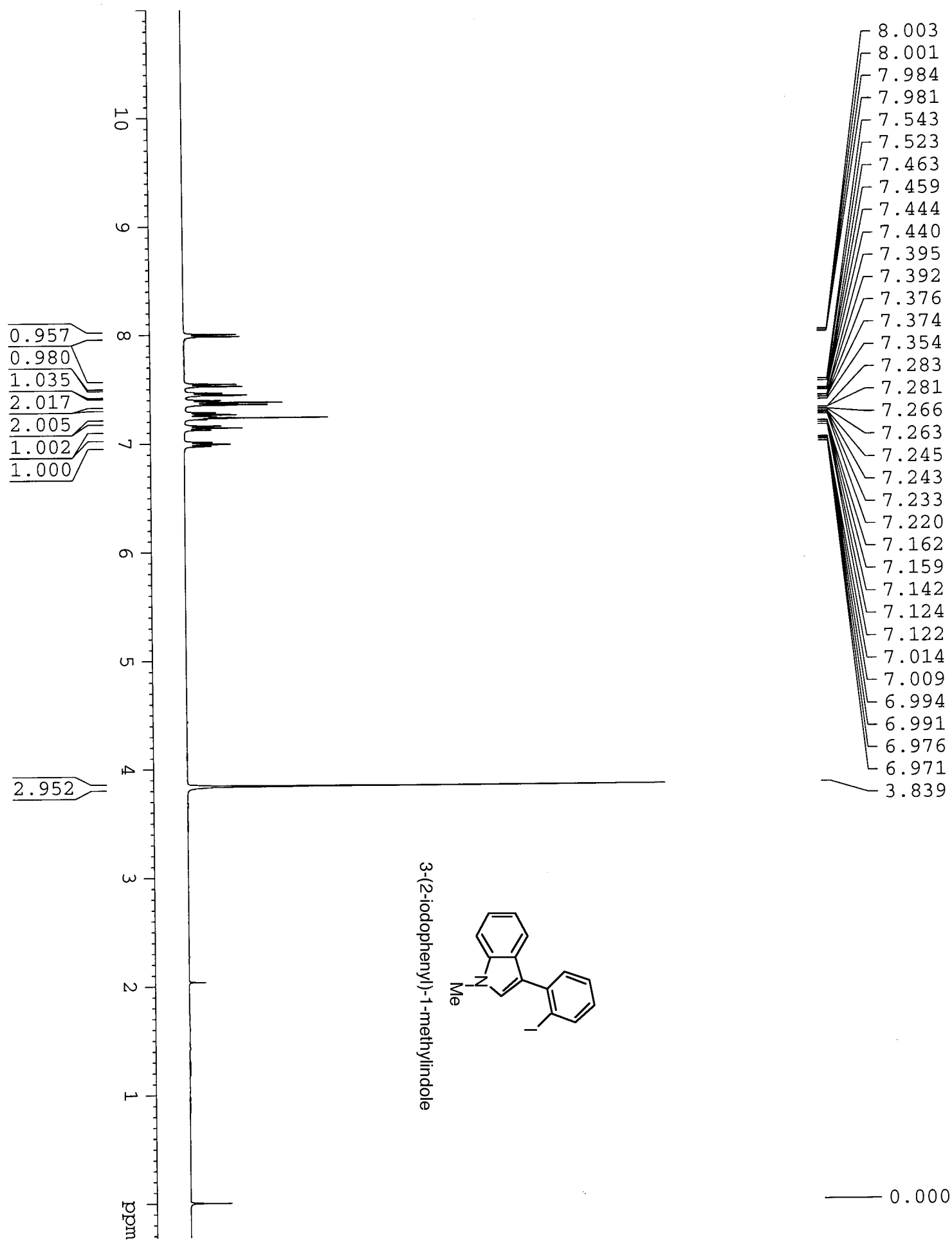

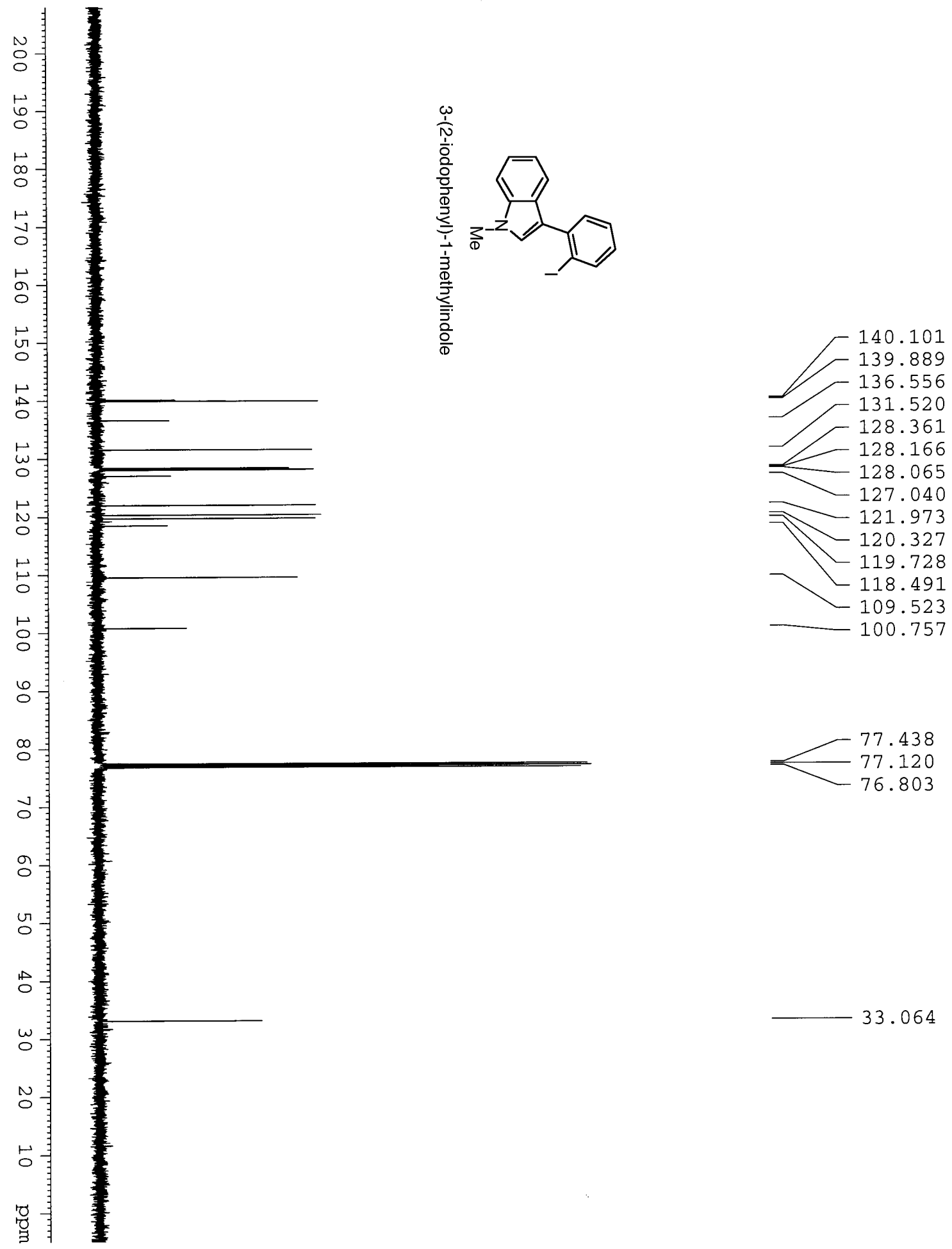

33.064 

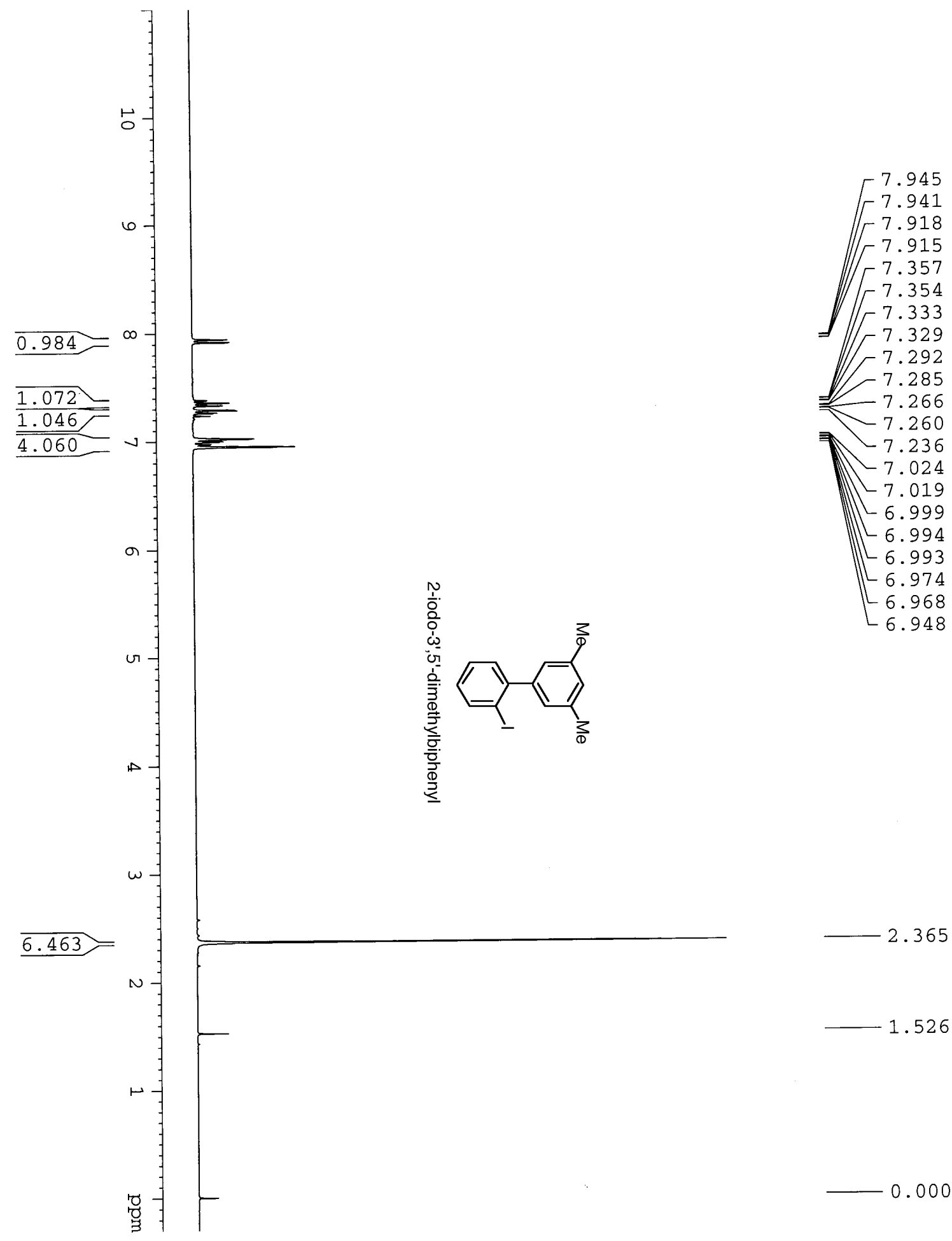

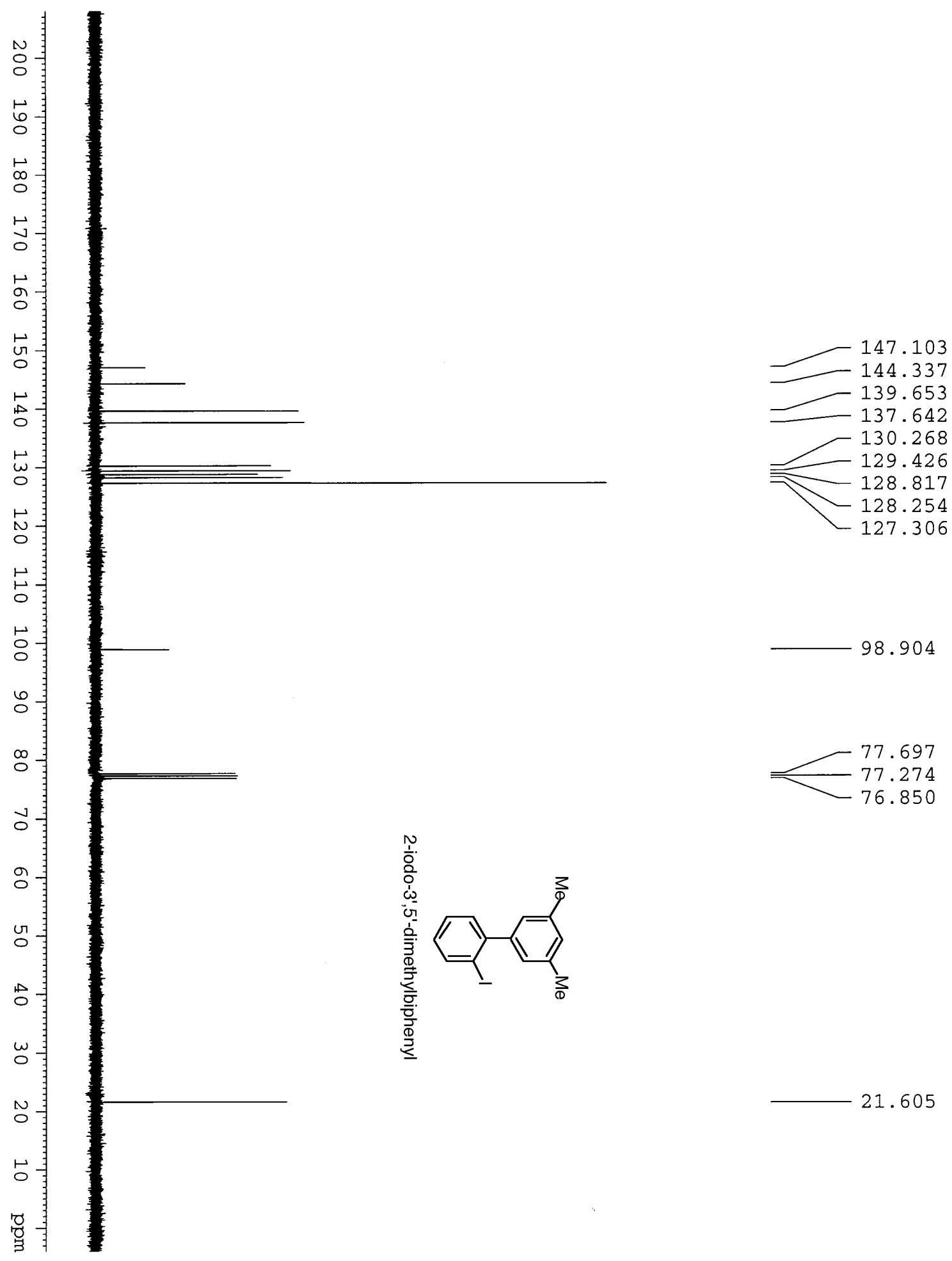

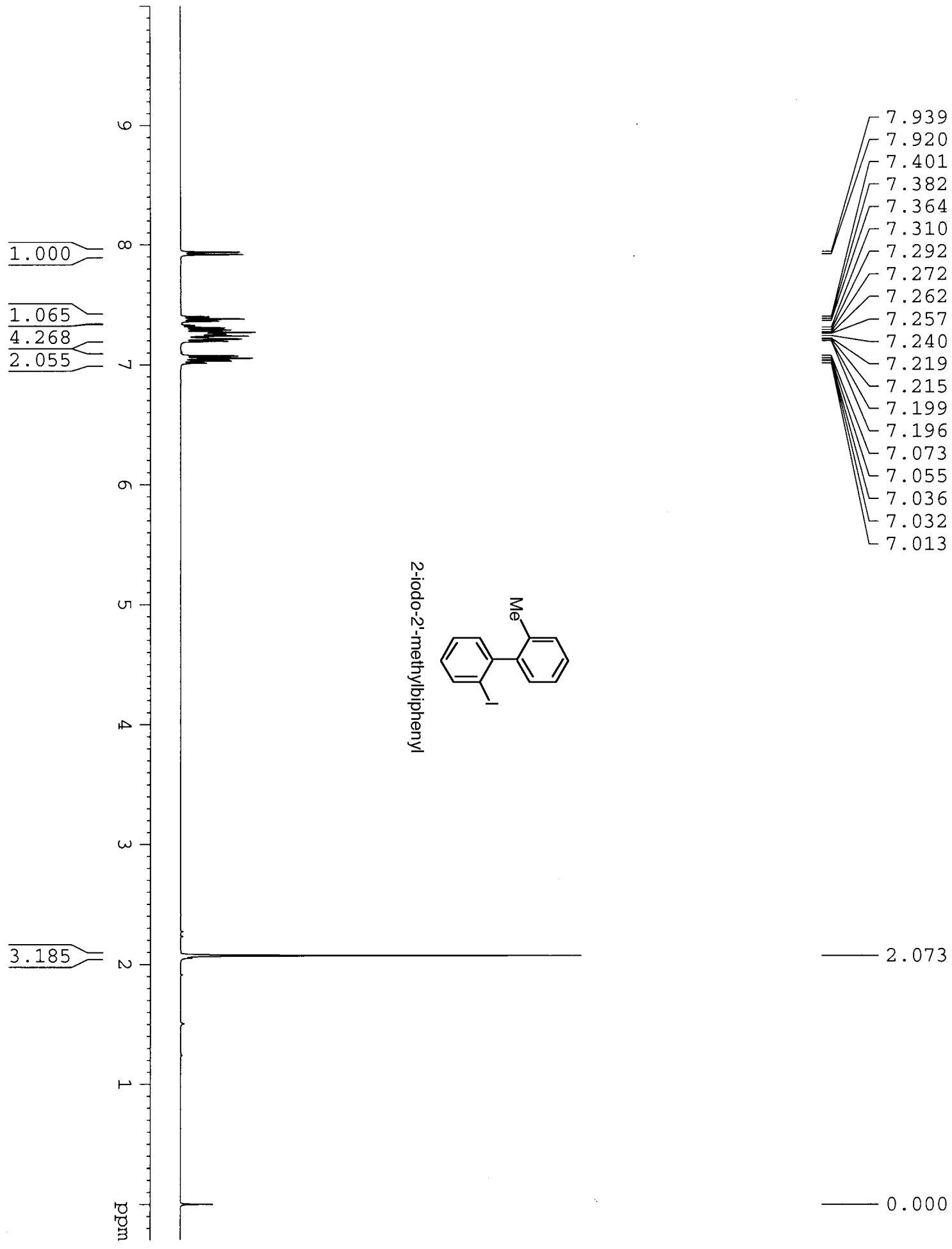

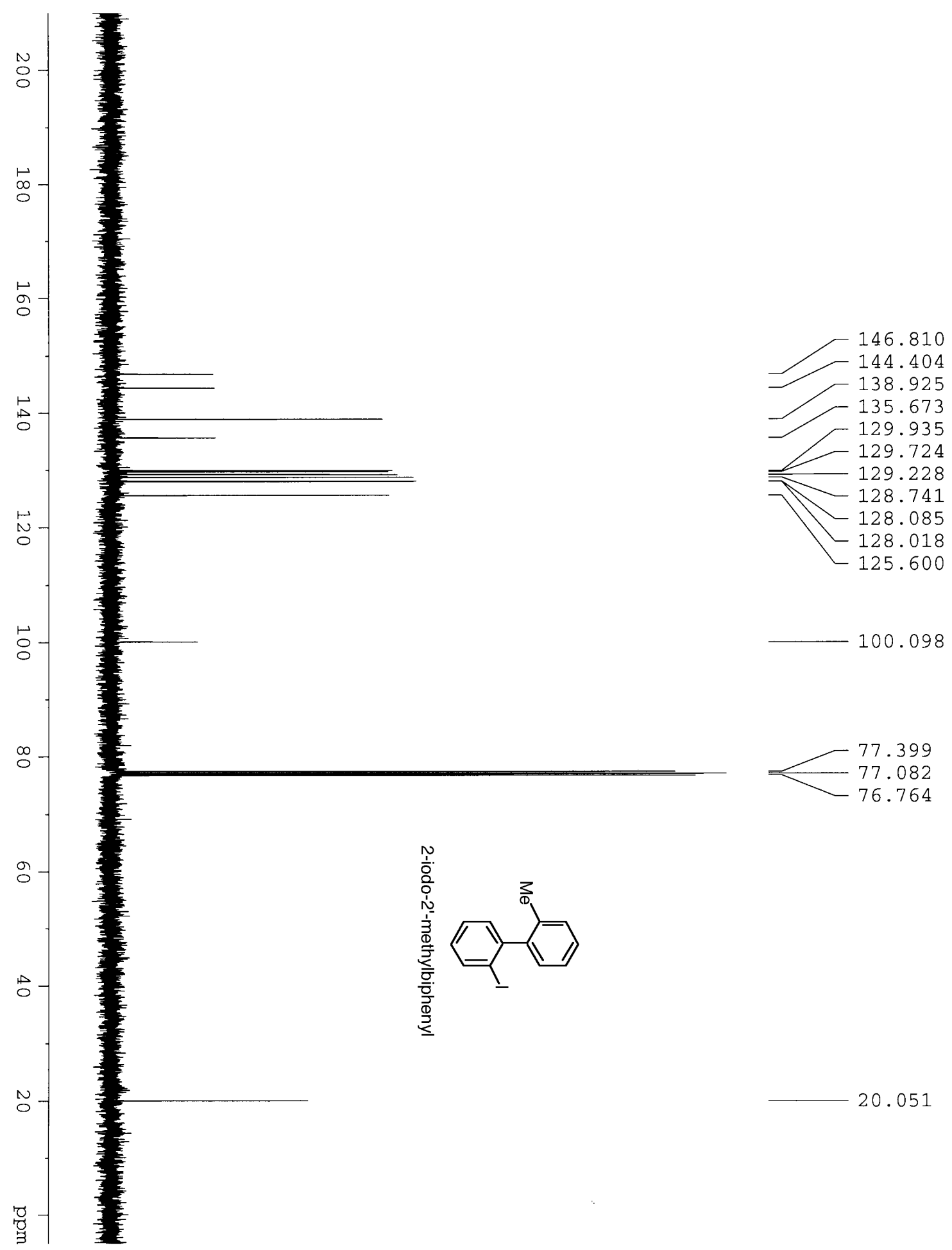

100.098

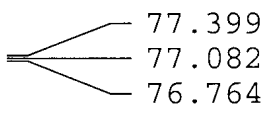

20.051 

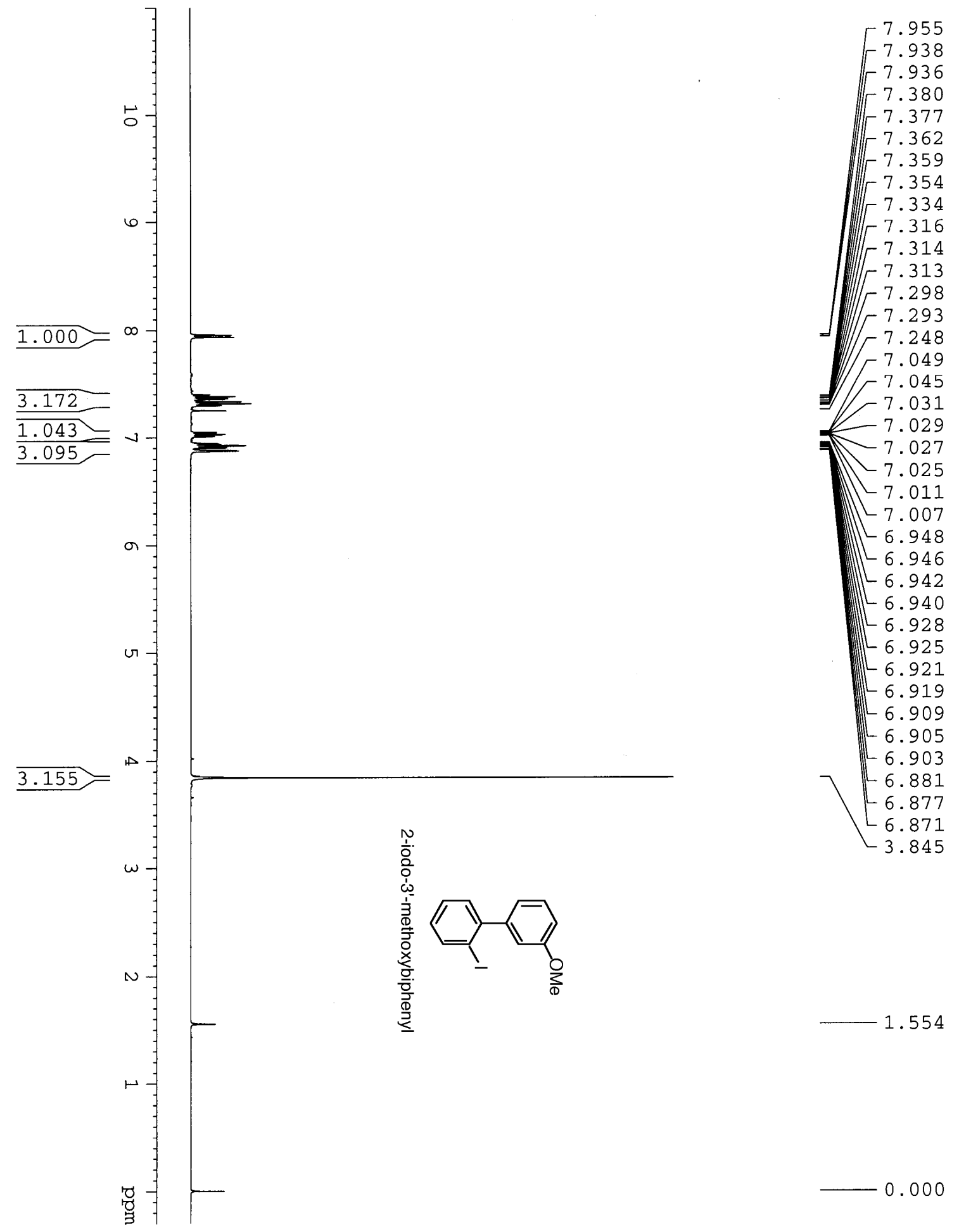

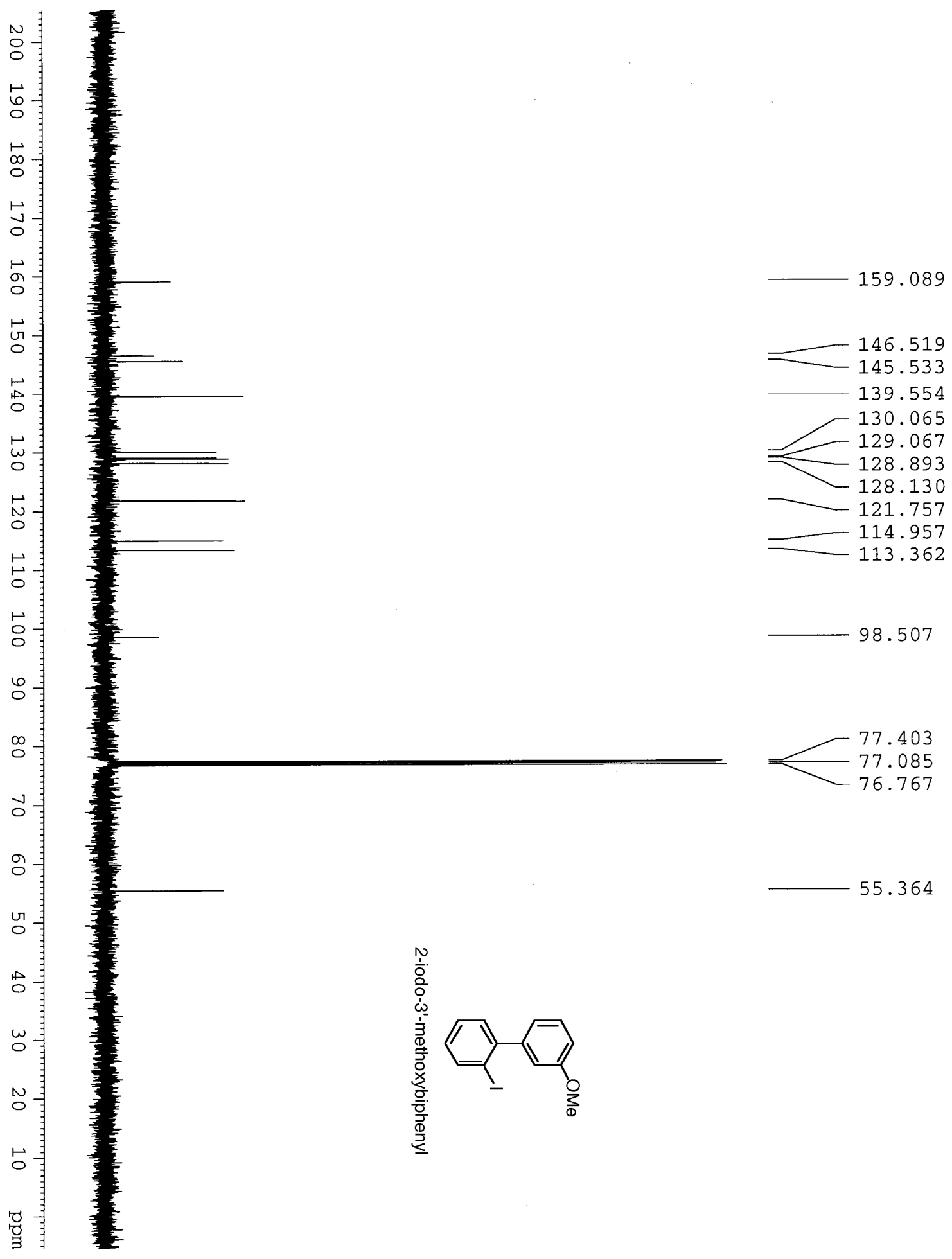

98.507

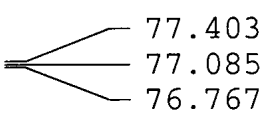

55.364 


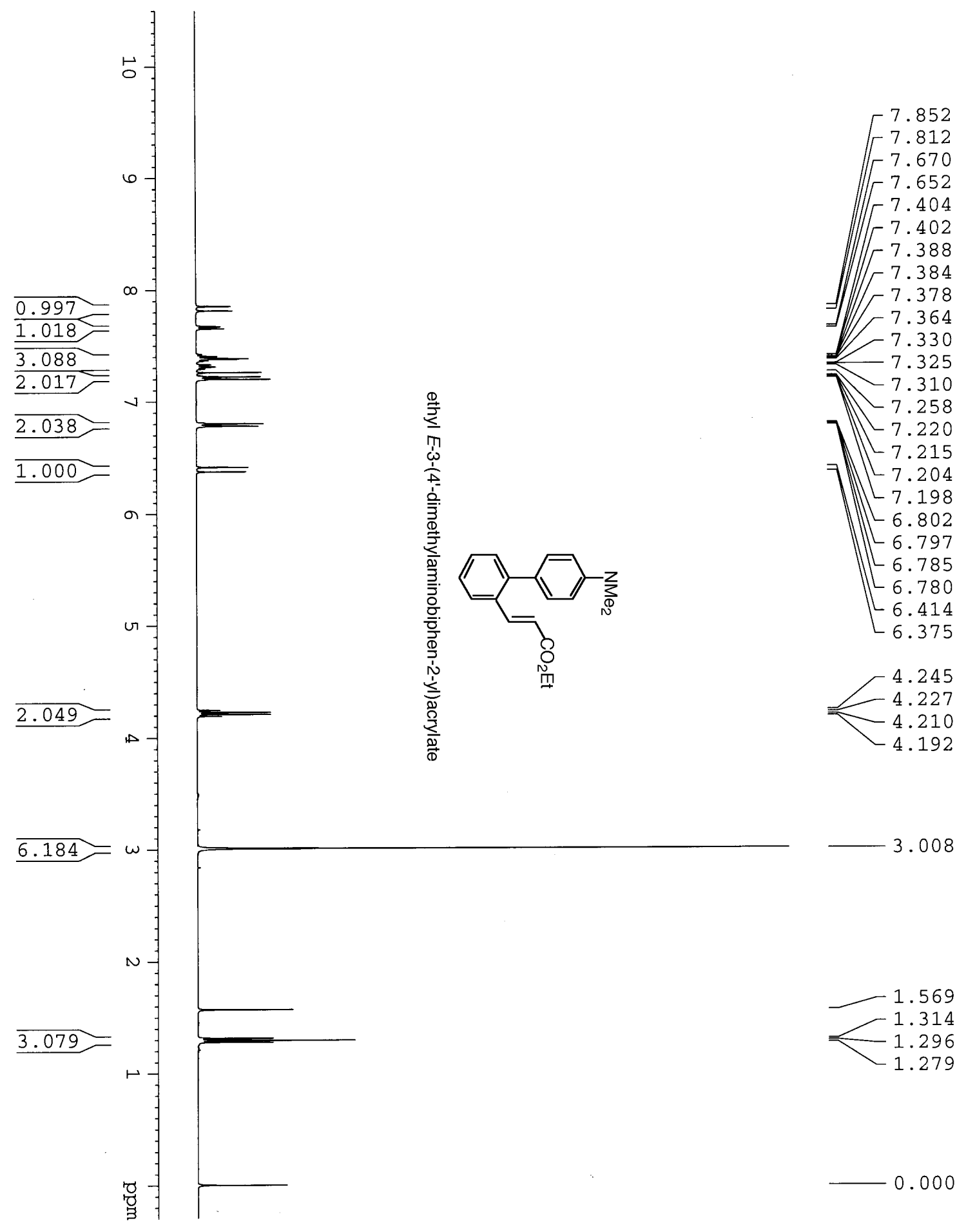




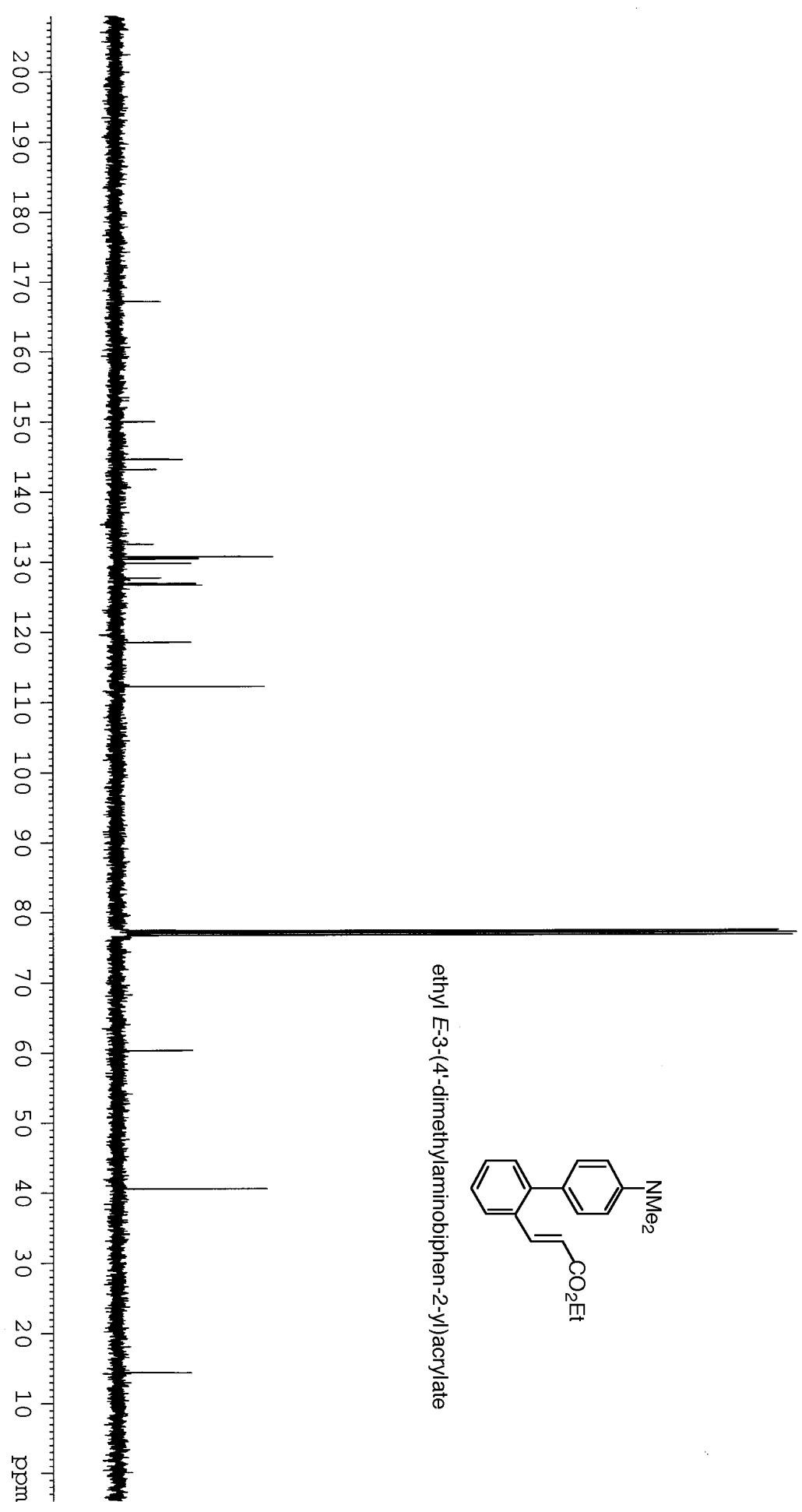
167.173
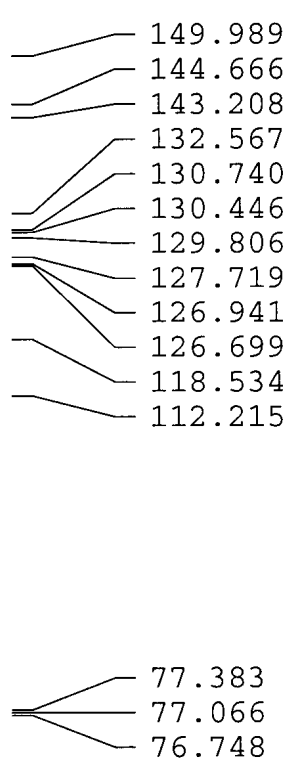

76.748

60.326

14.380 


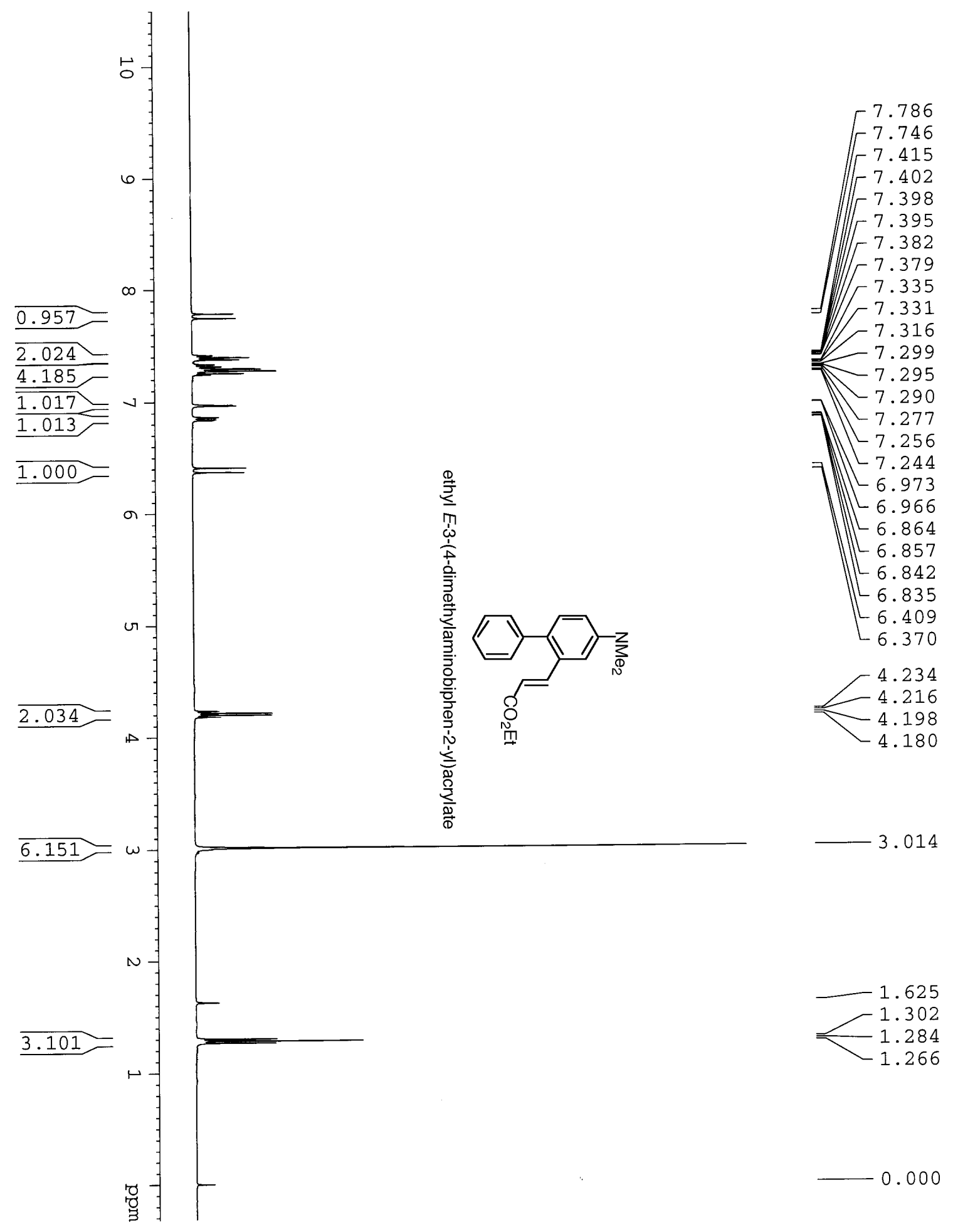



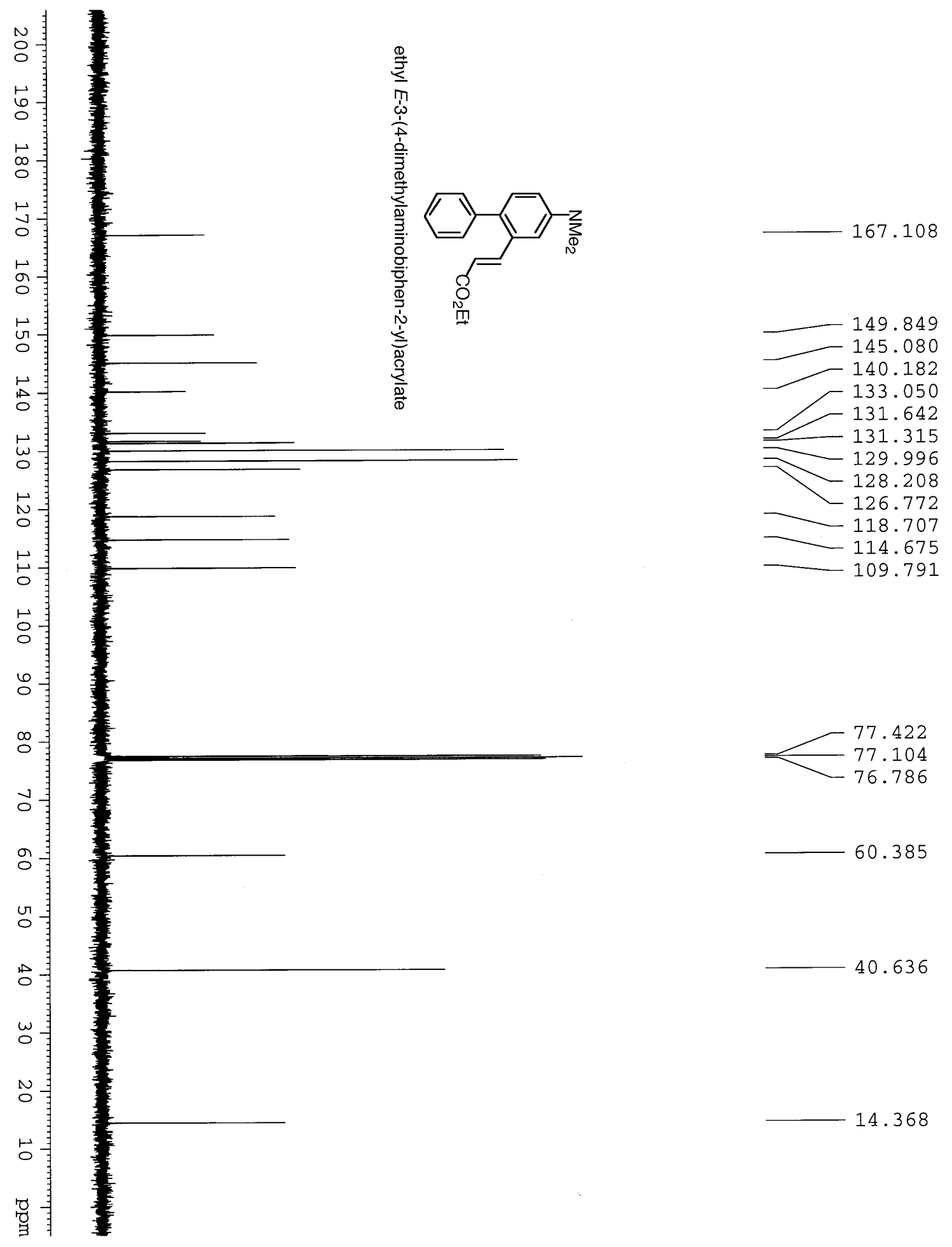

60.385

40.636

14.368 

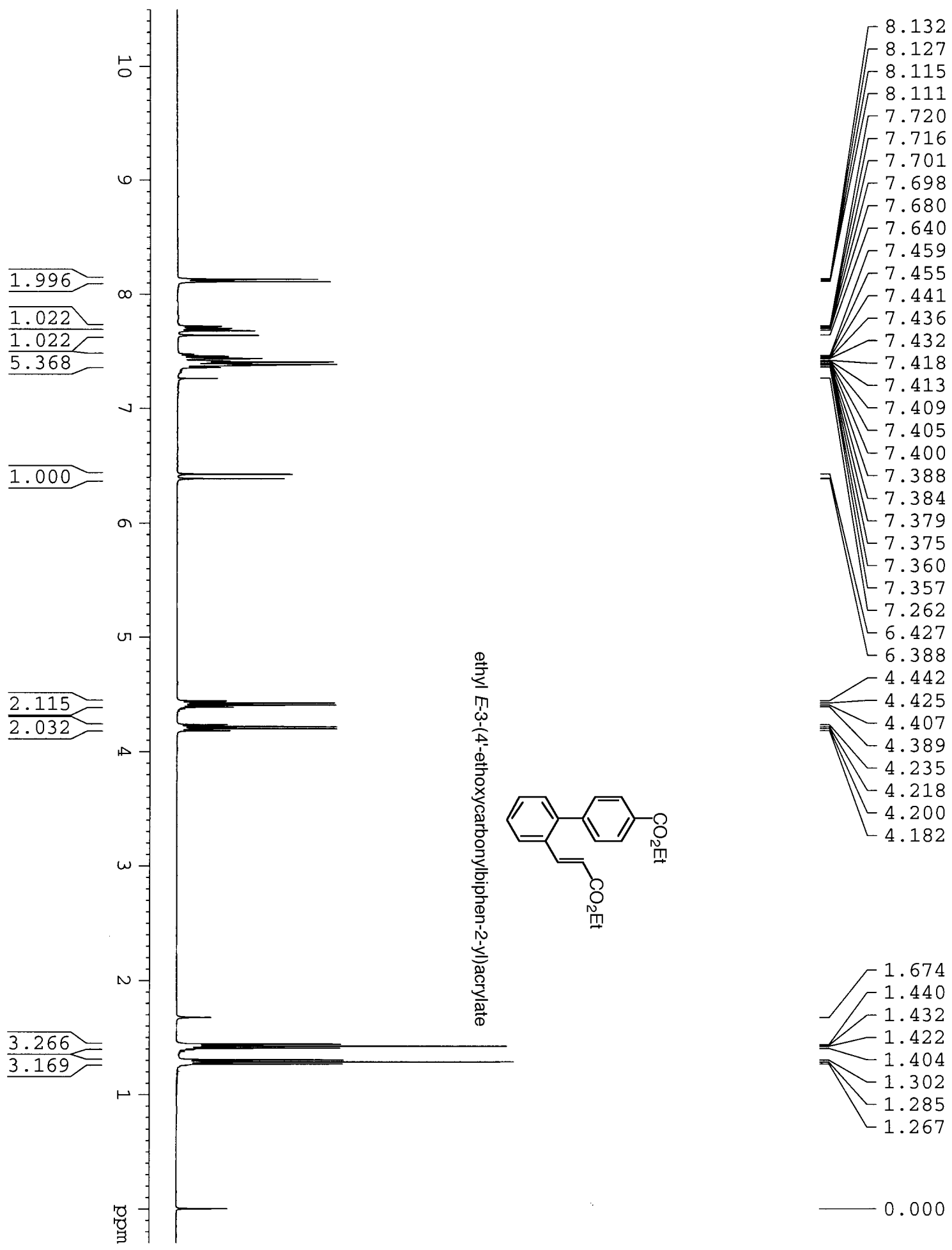

0.000 

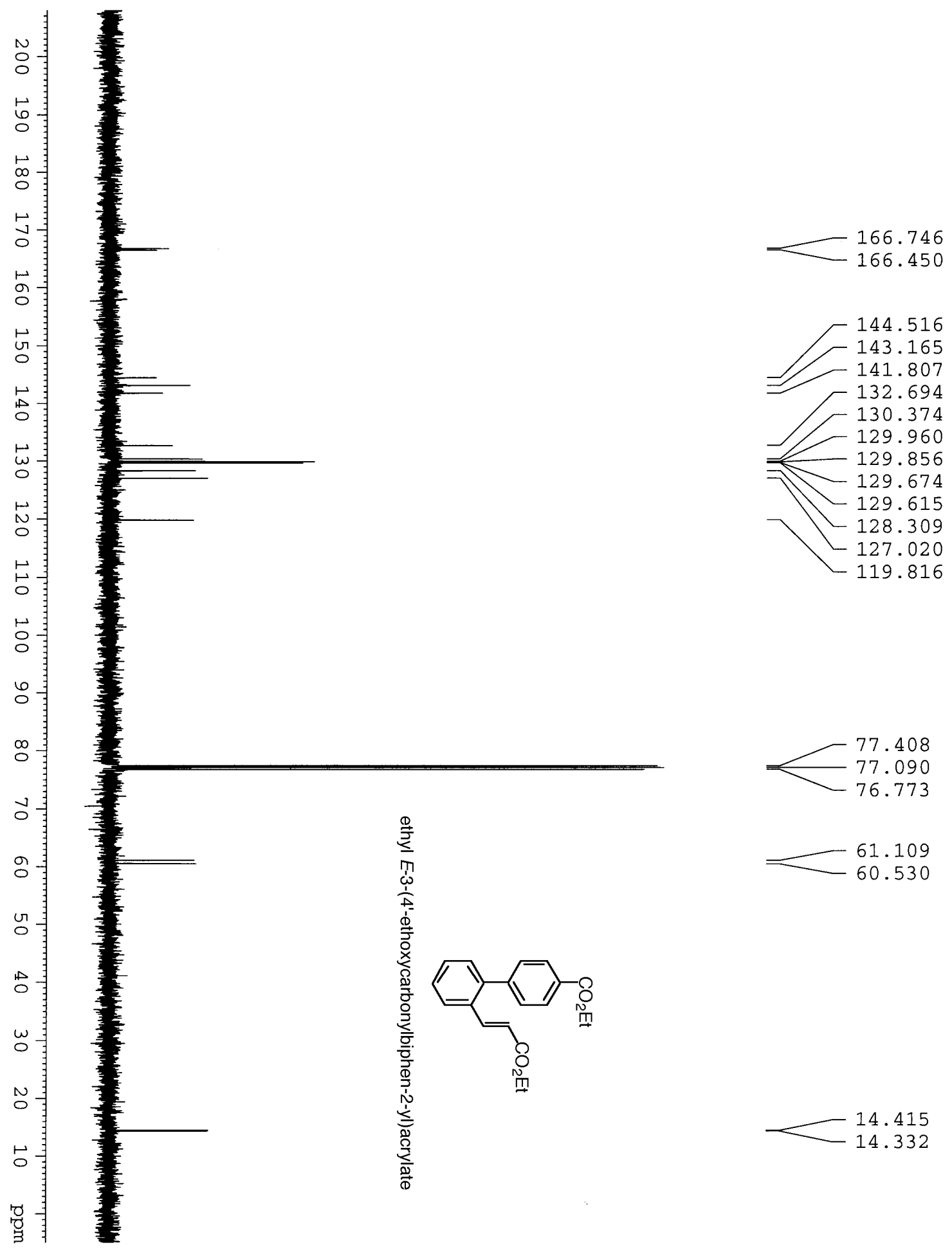

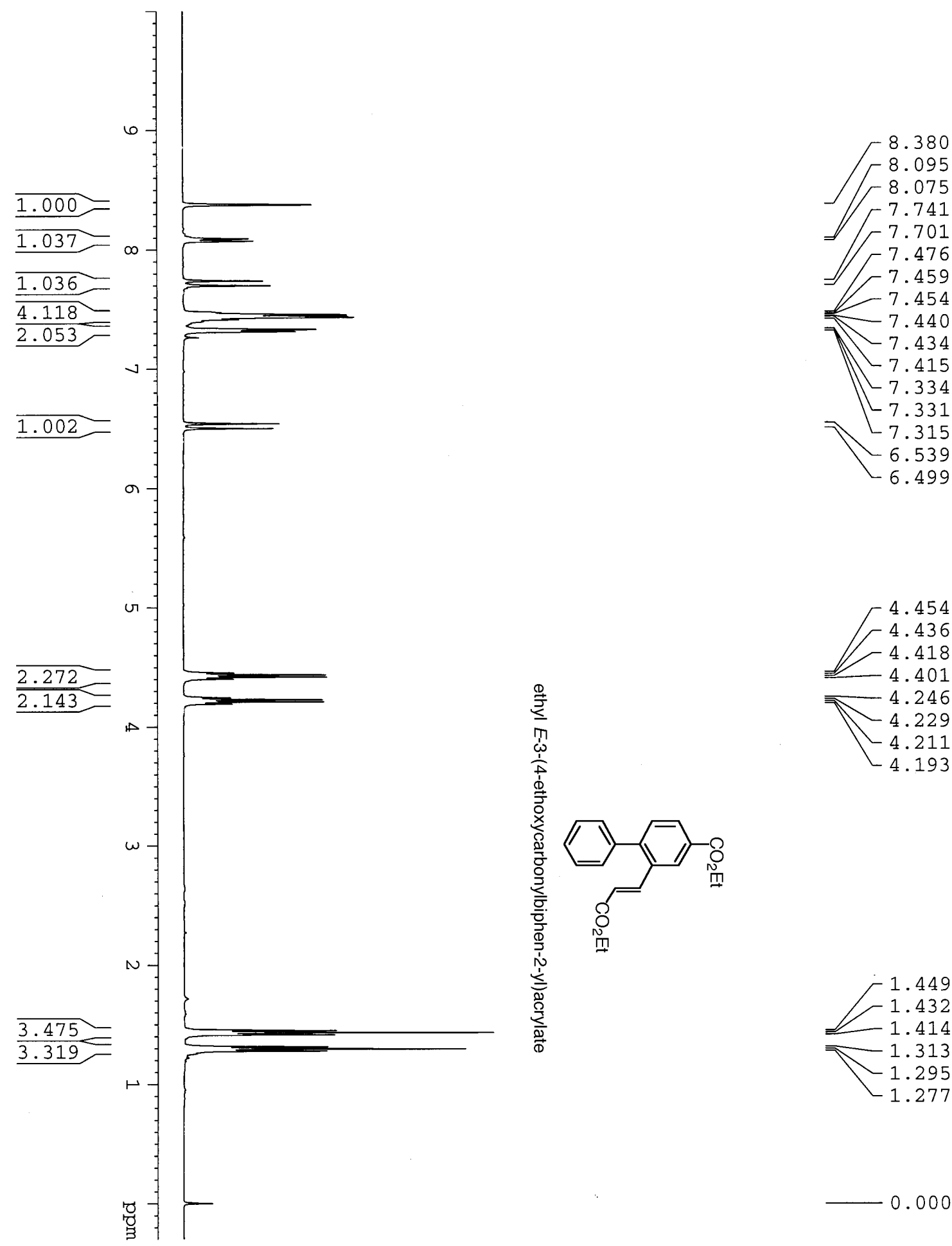

0.000 

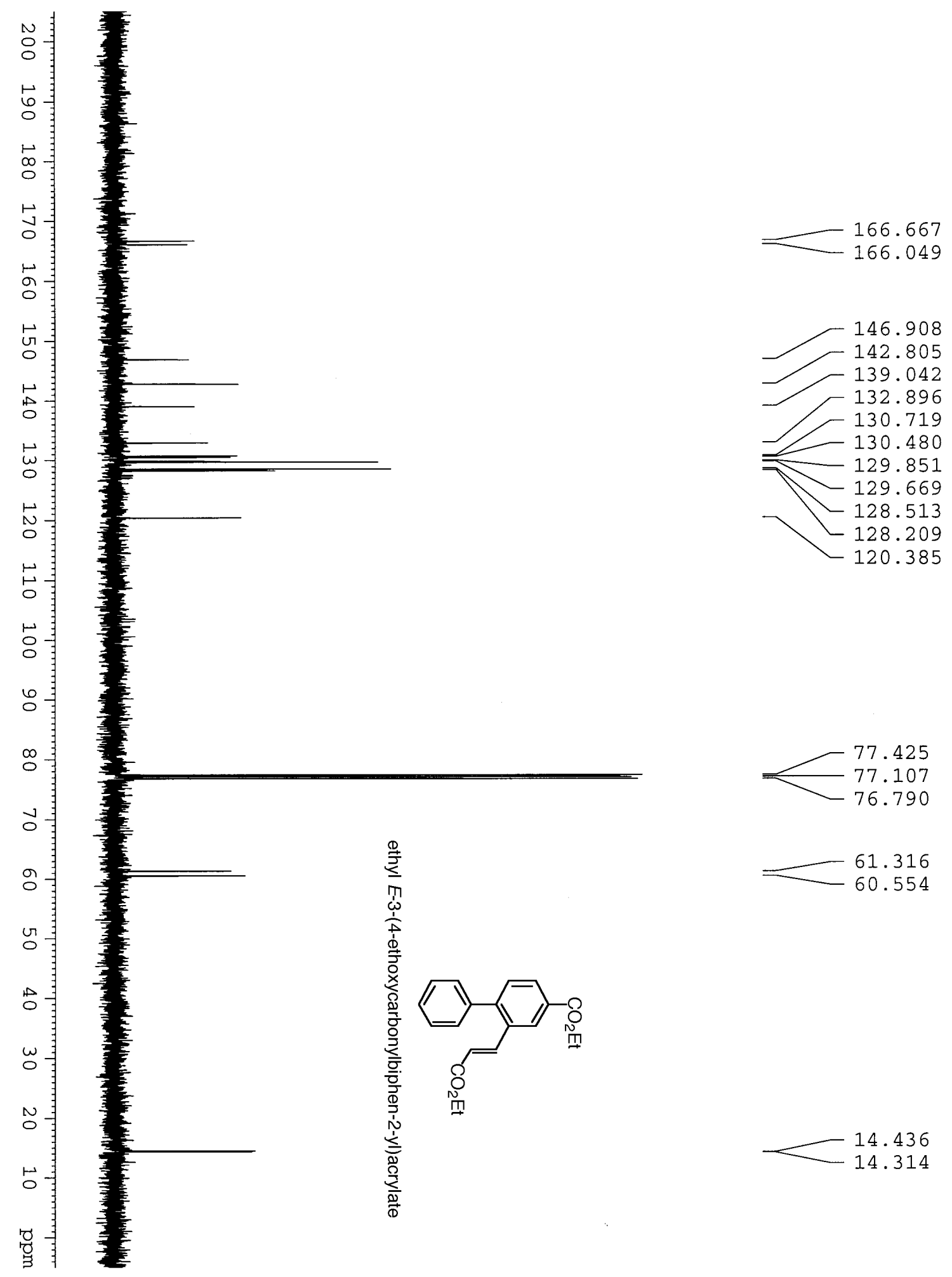

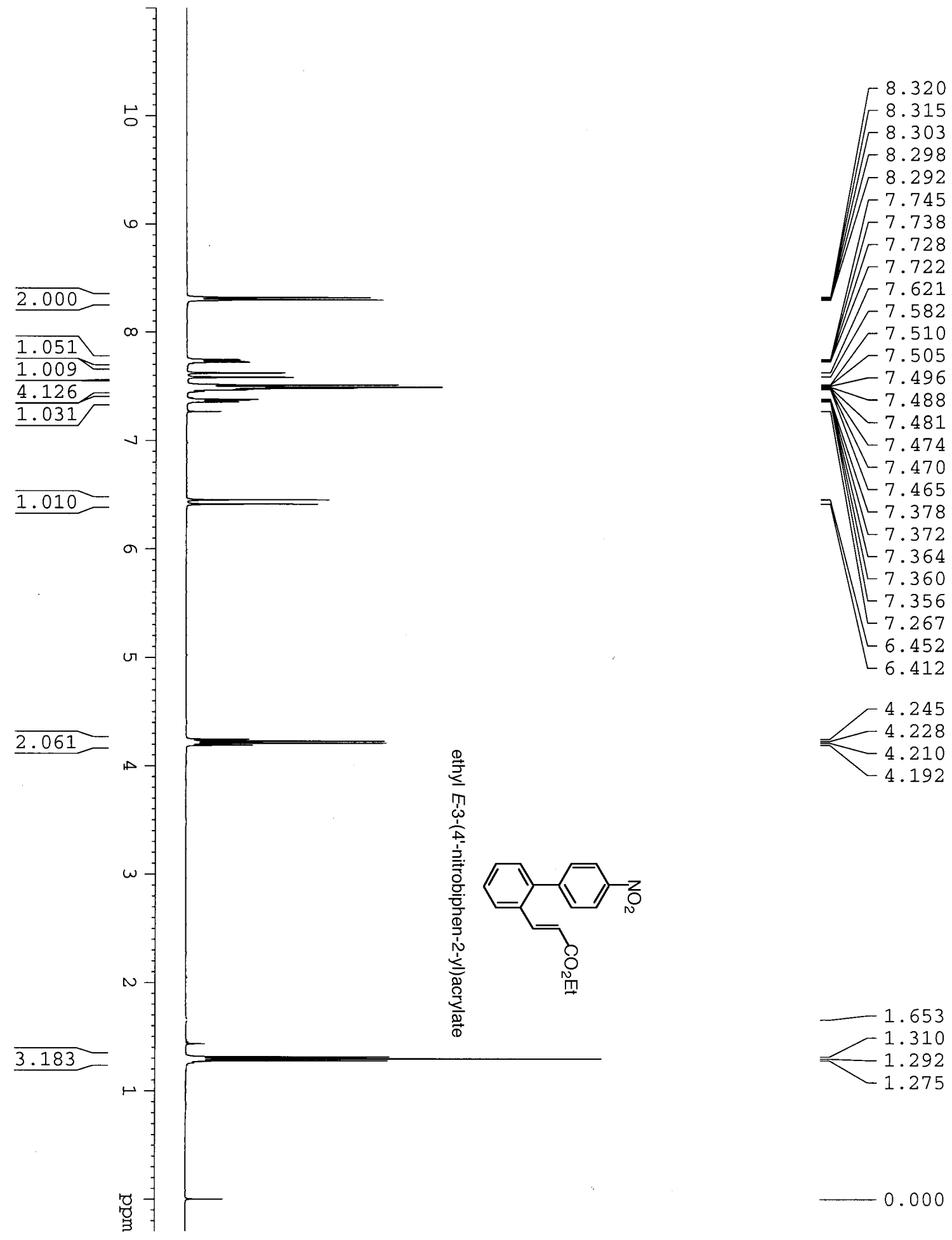

$-0.000$ 


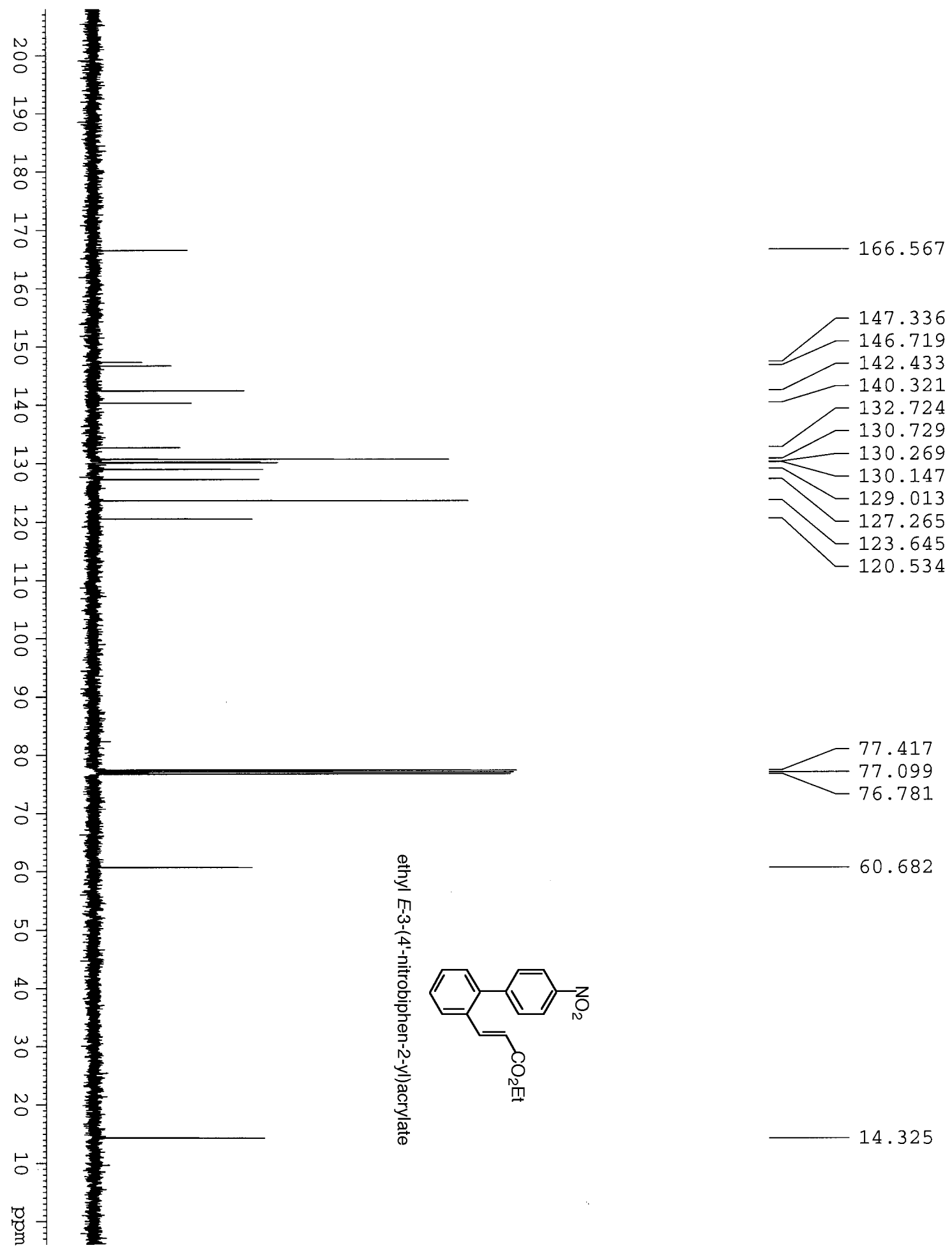



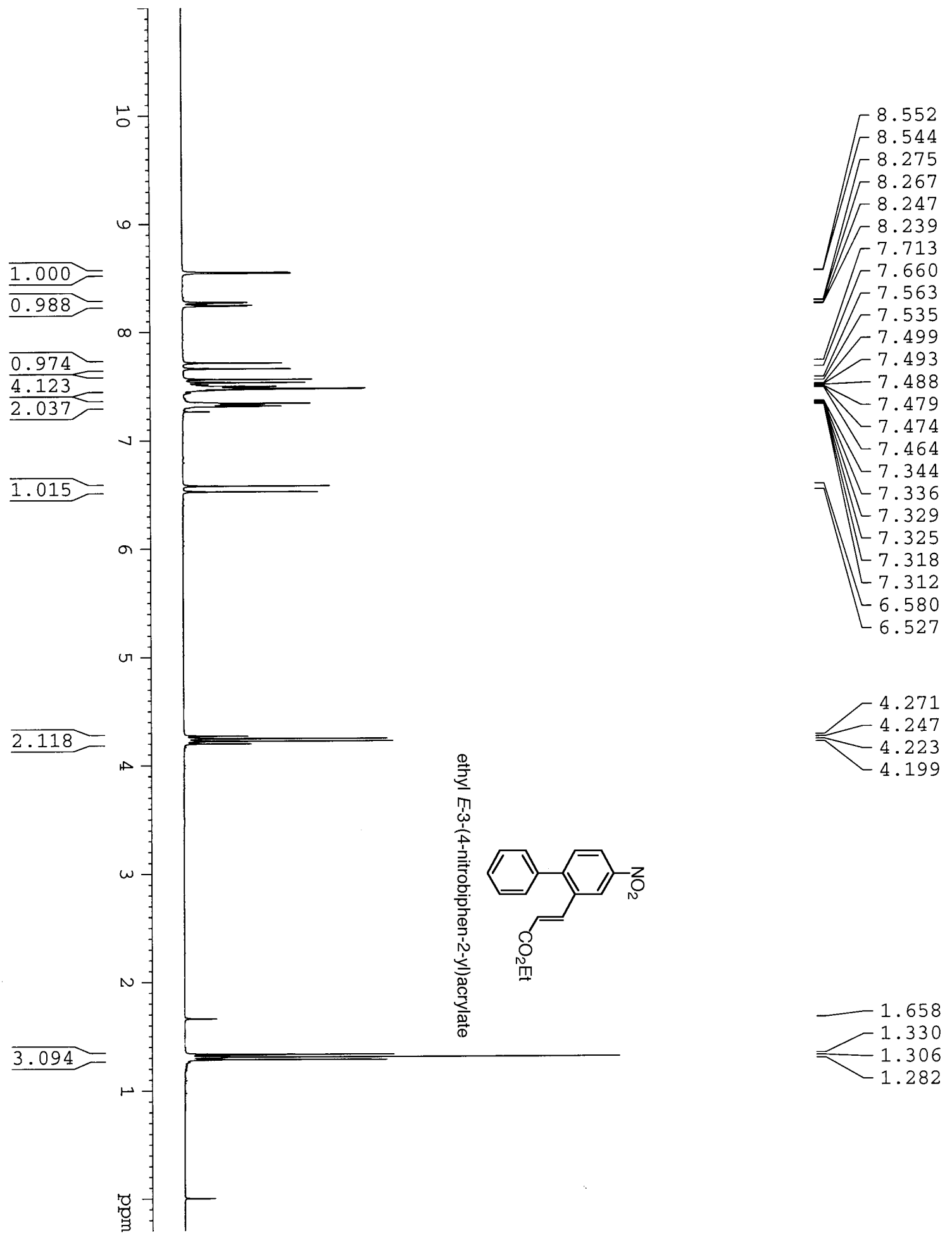


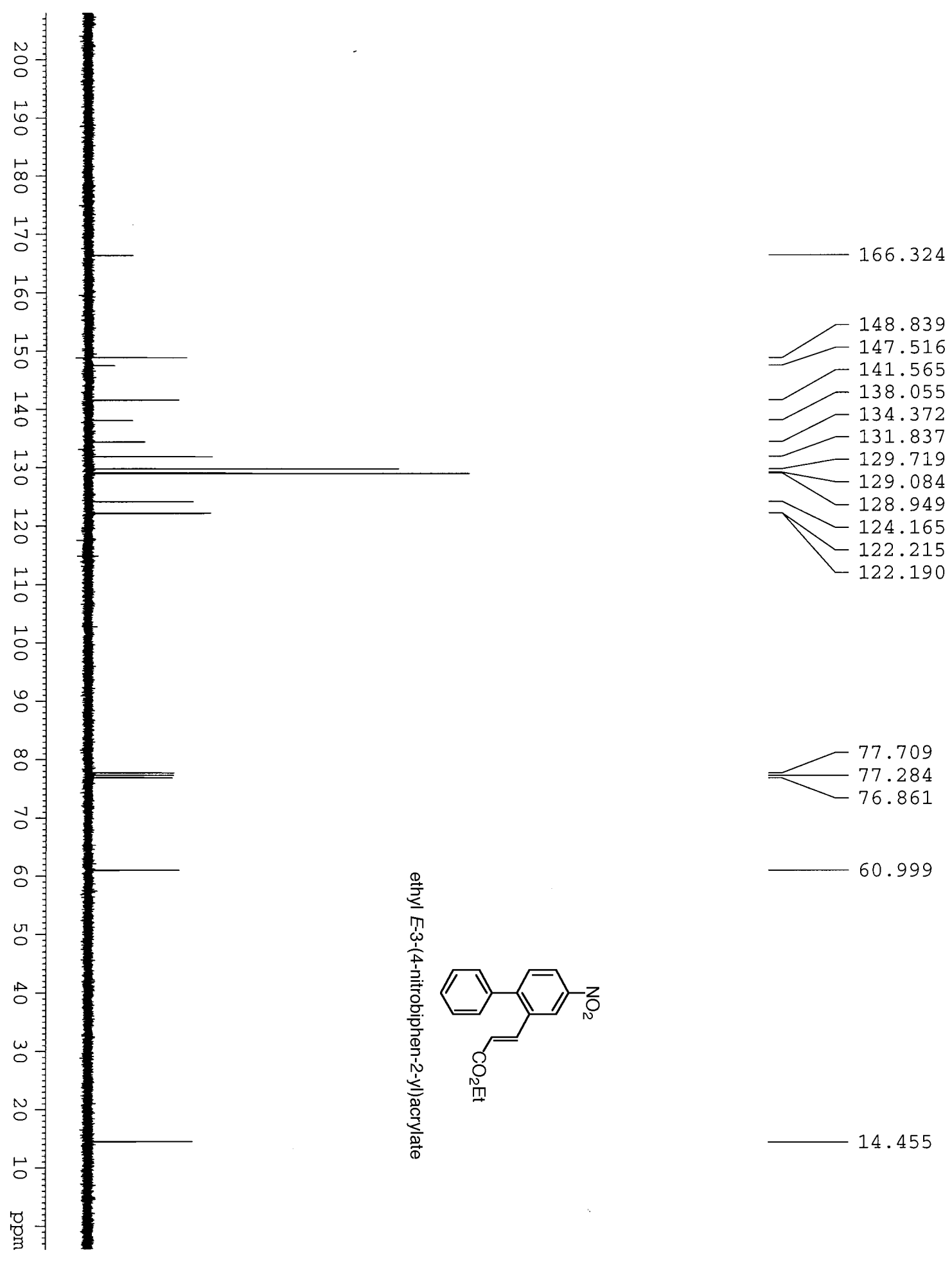




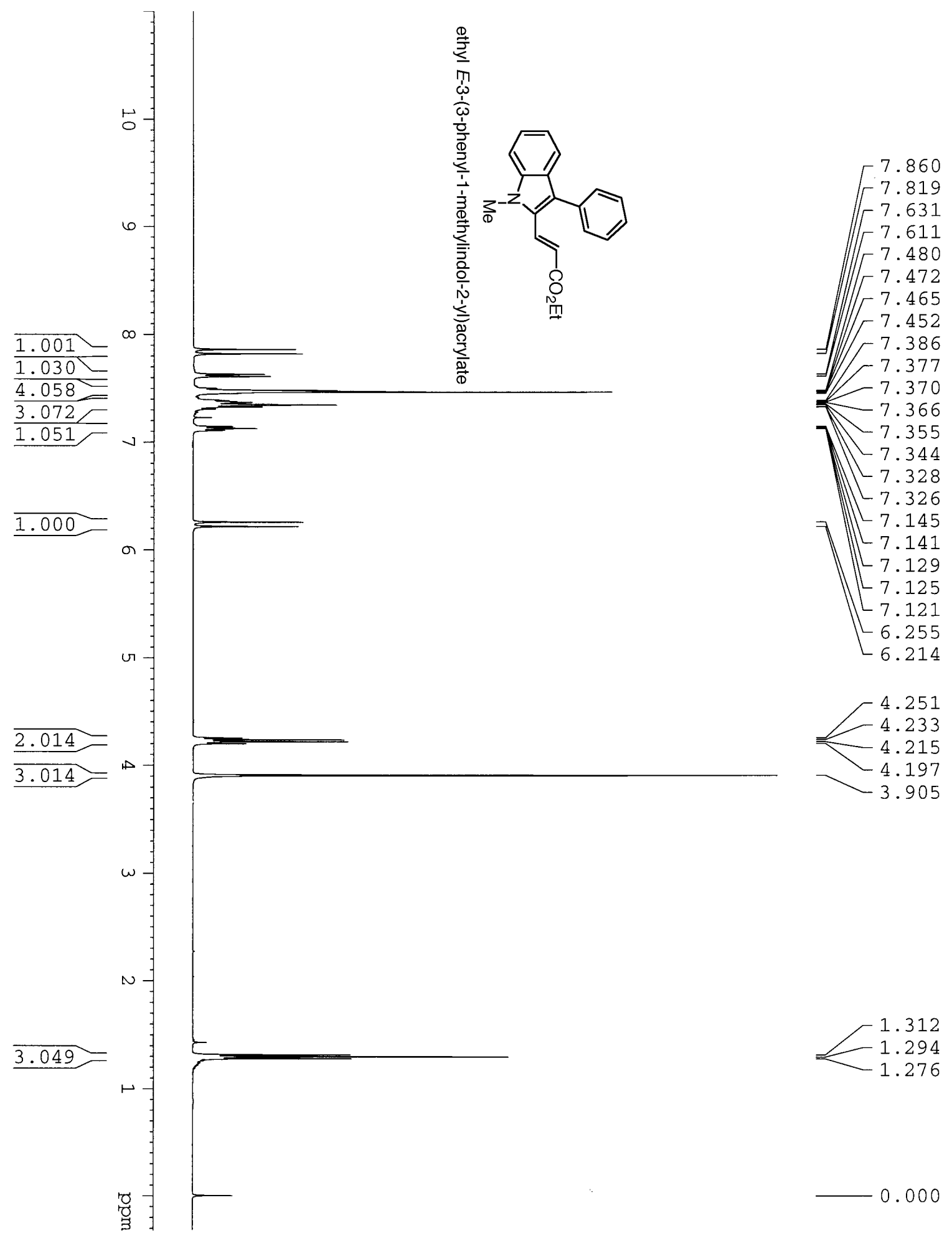




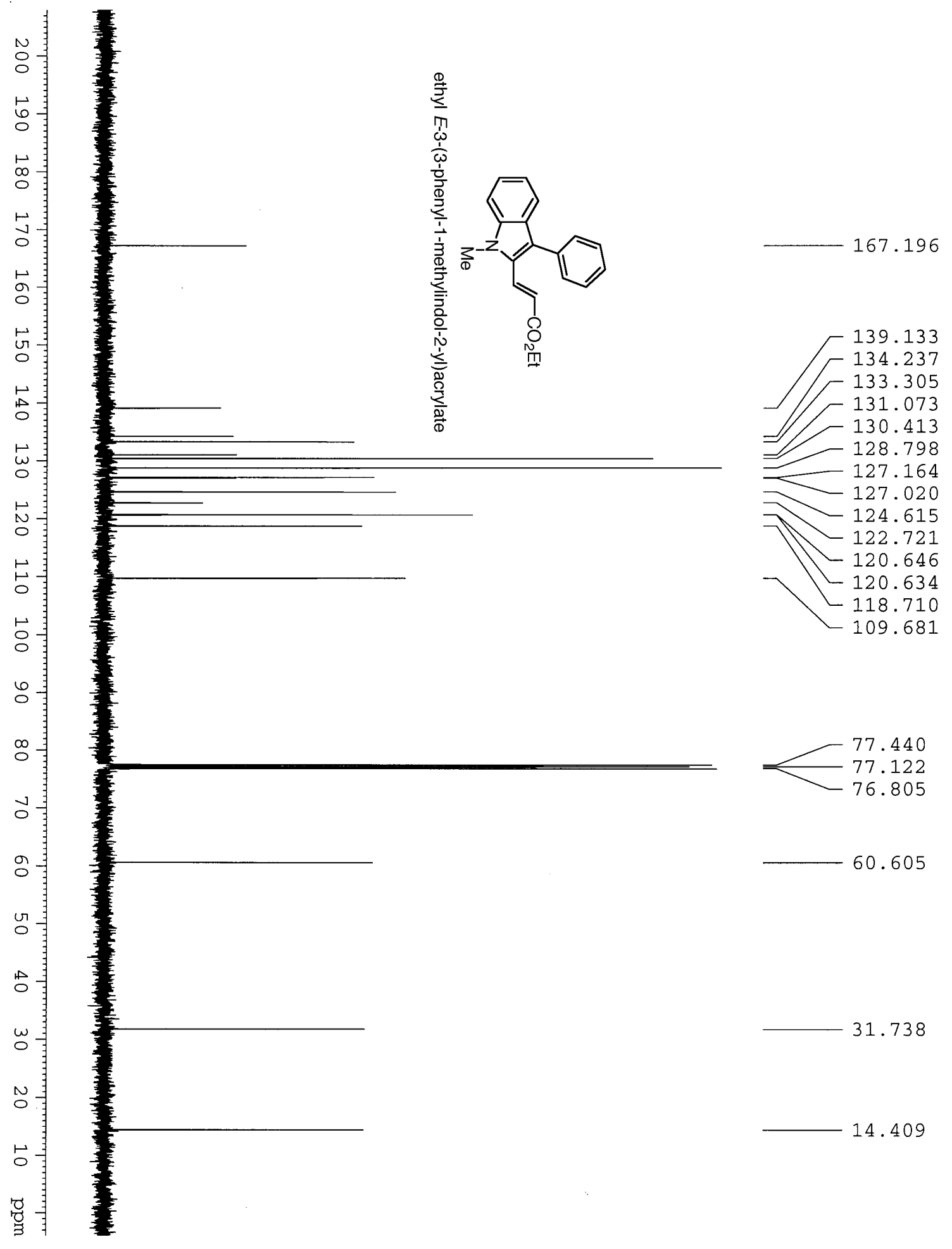



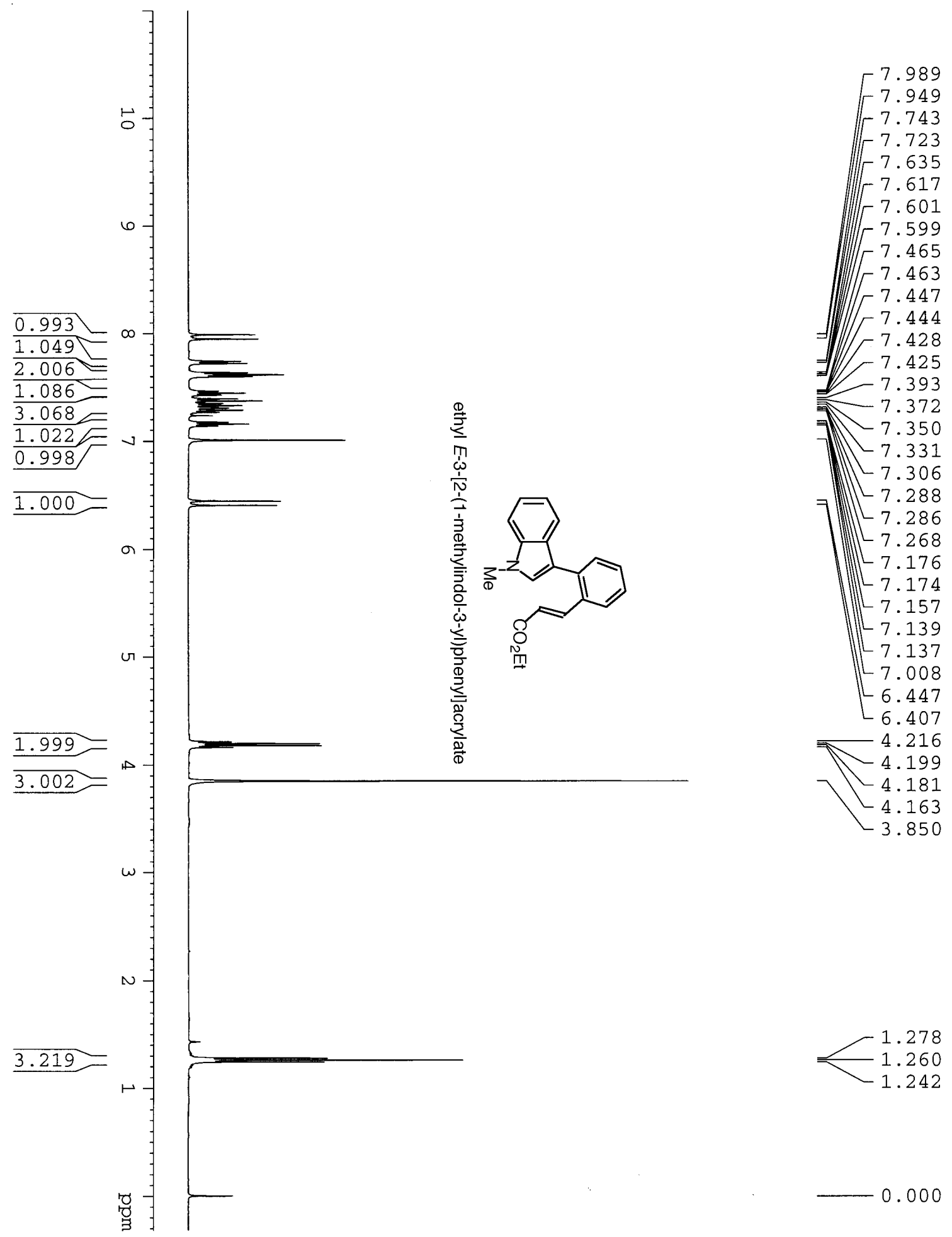

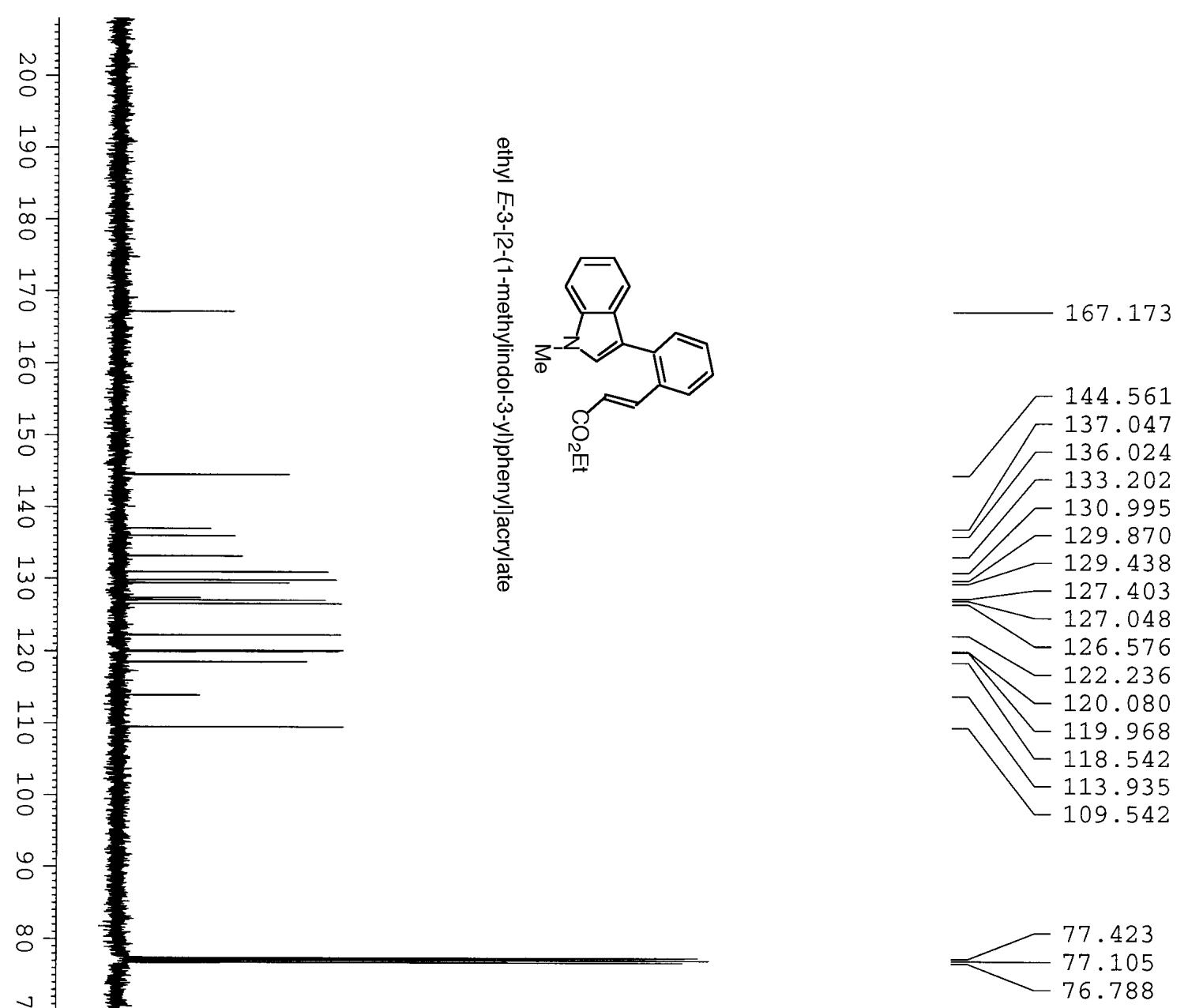

o

$\stackrel{\infty}{\circ}$

菃

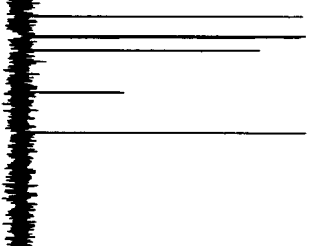

$\circ$

搽

on

ज

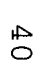

$\stackrel{w}{\circ}$

N

$\vdash$

$\circ$

弚

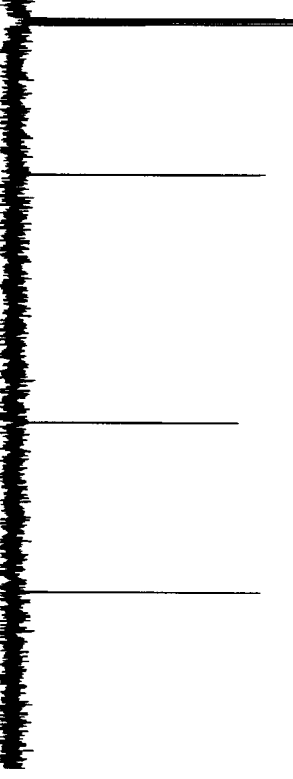



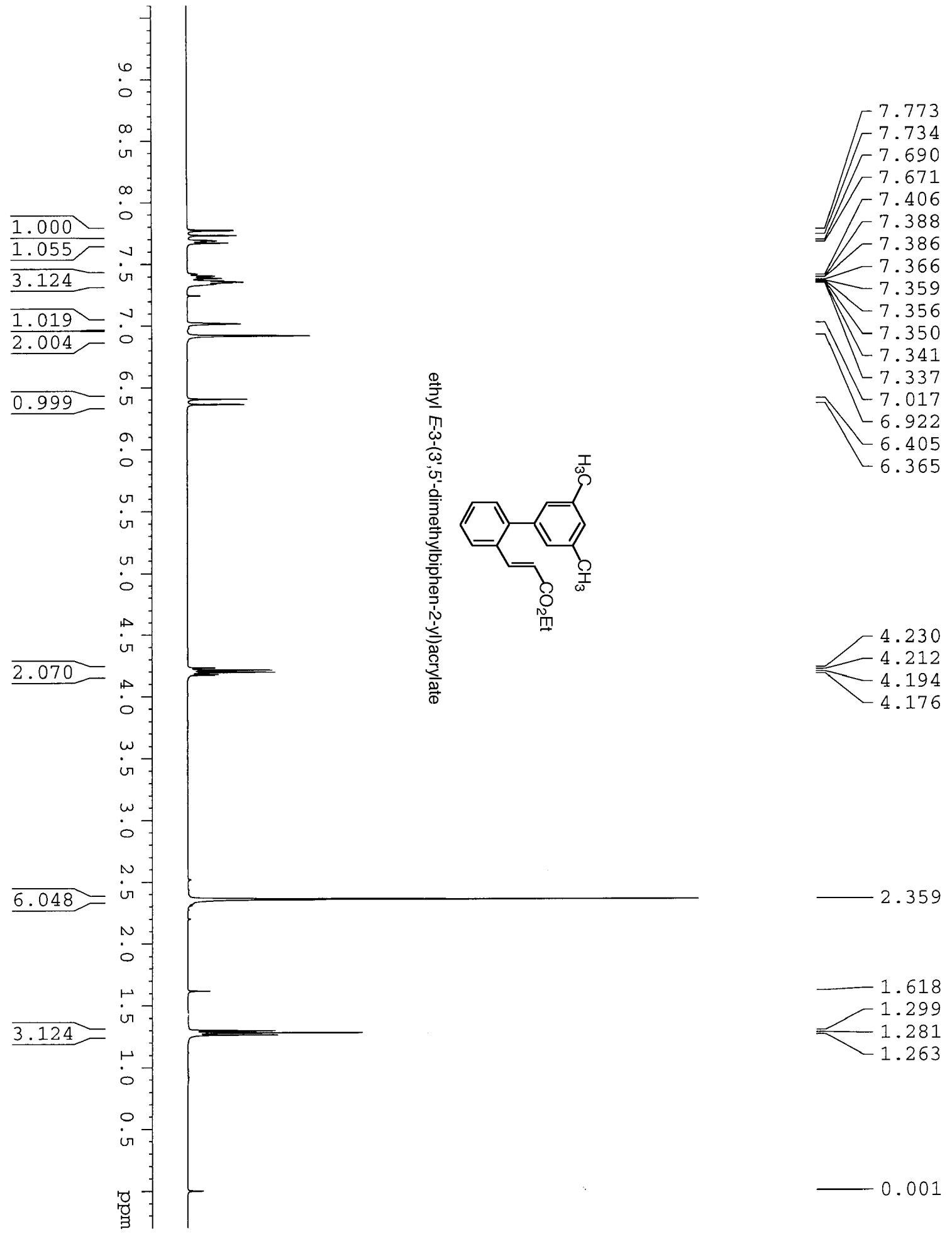

4.230

$-4.212$

4.194

2.359

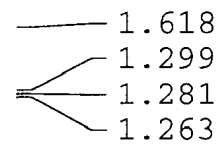

0.001 


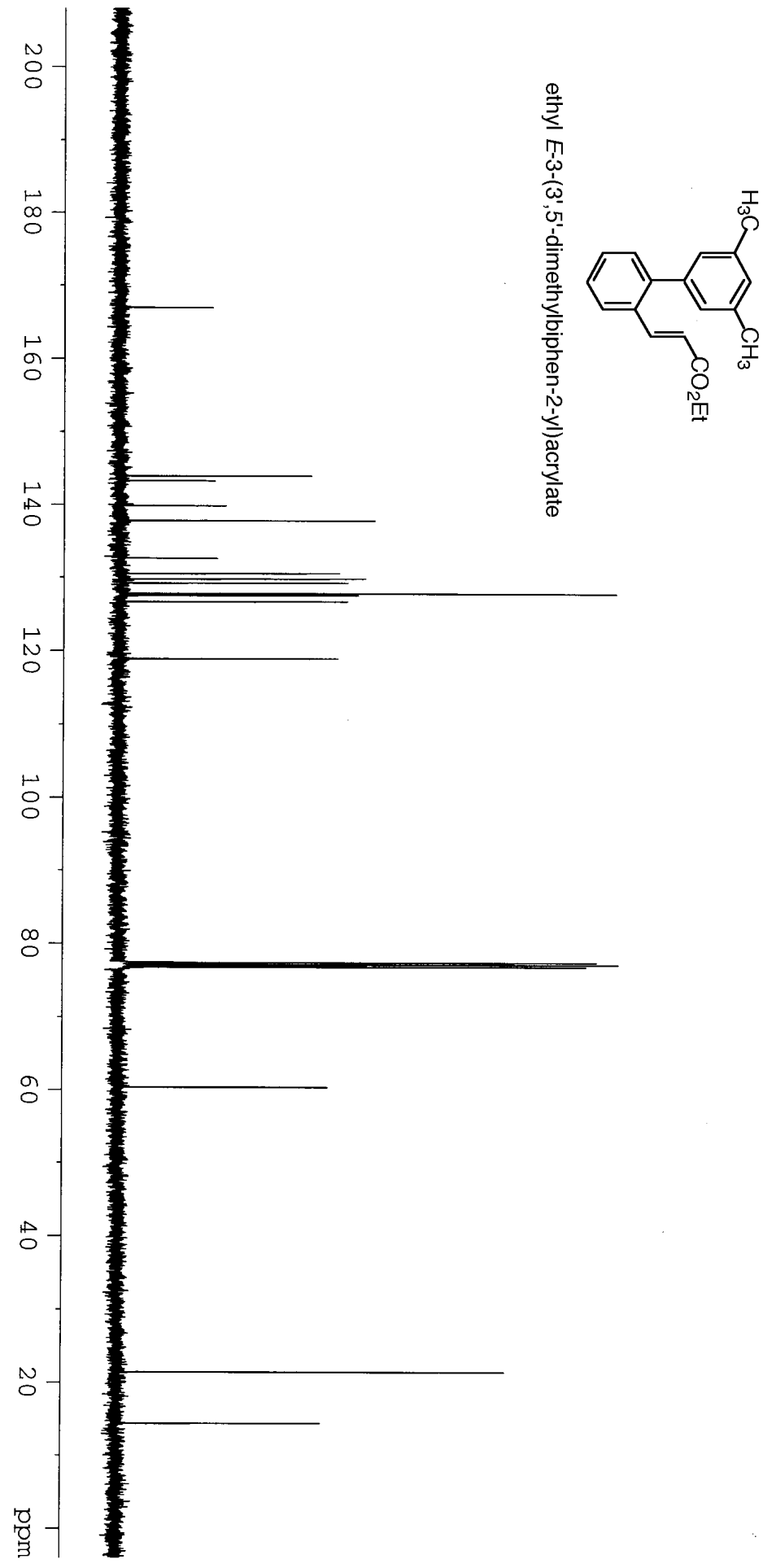

166.956
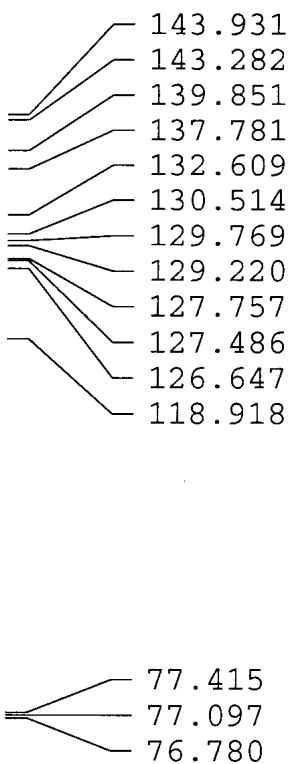

60.349

21.391

14.353 

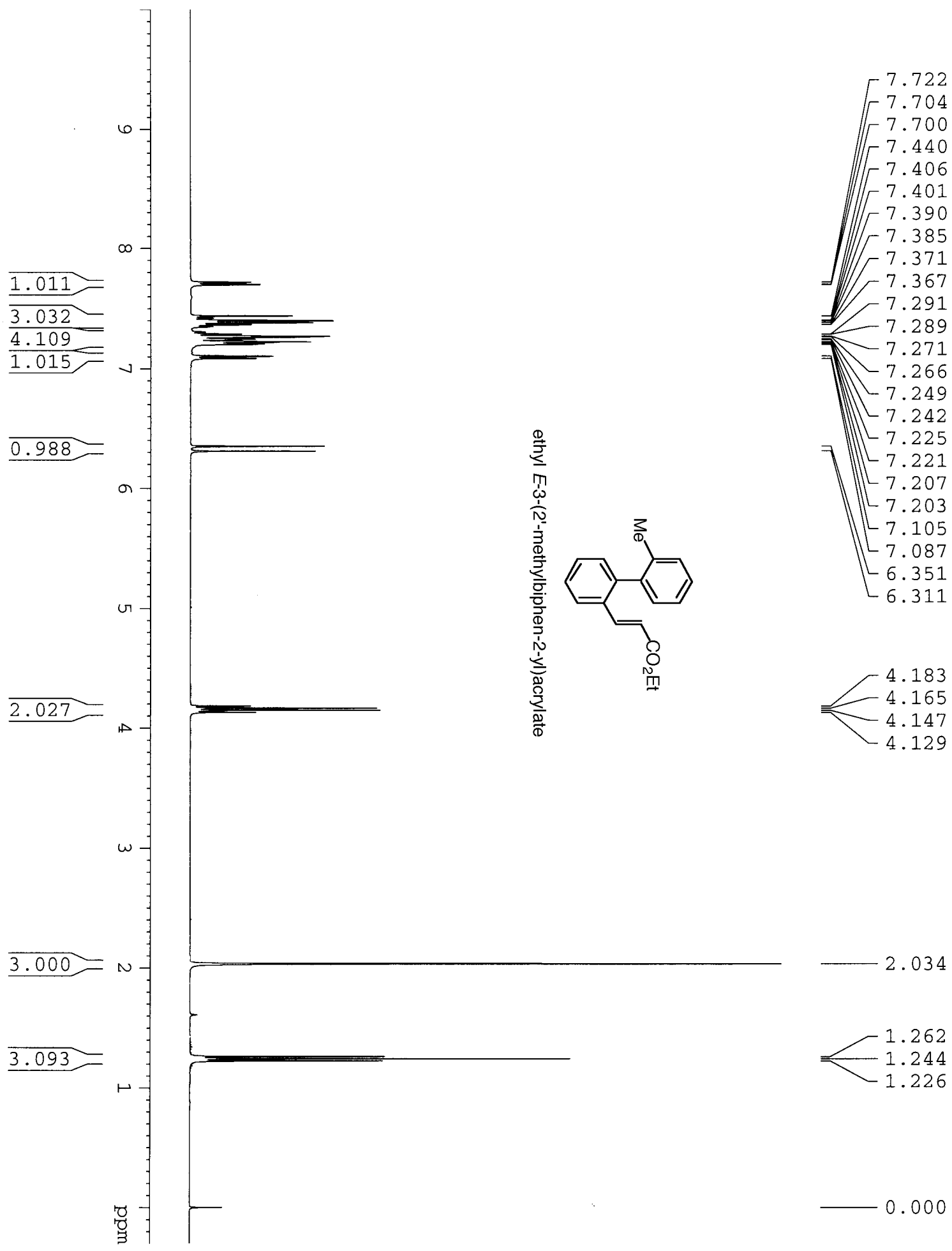

1.262

1.244

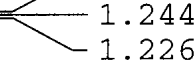

0.000 


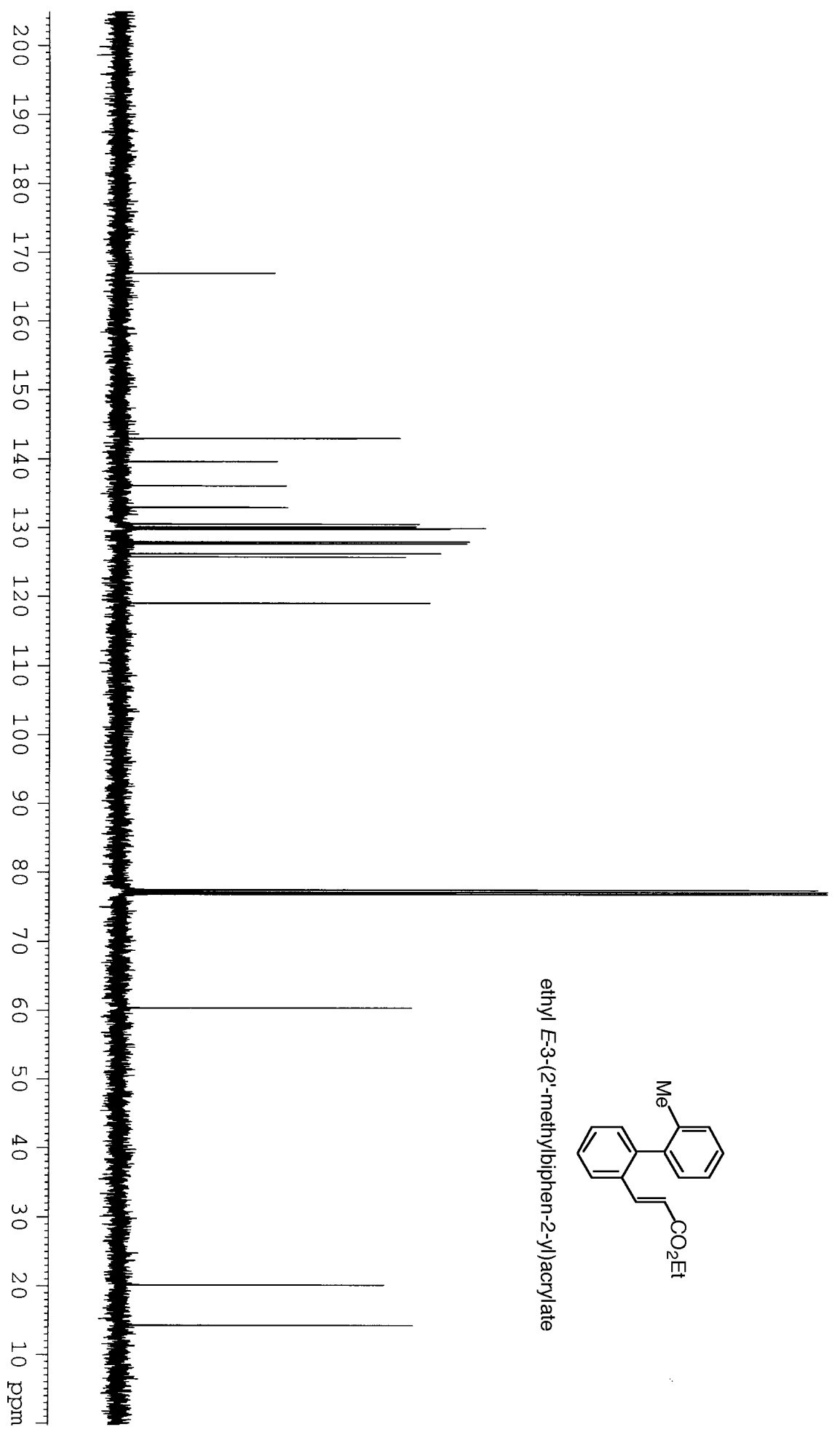

166.916
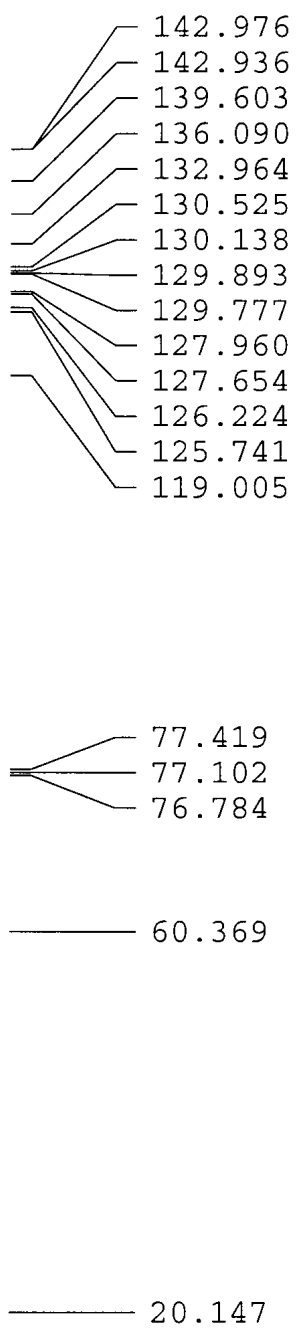

14.287 

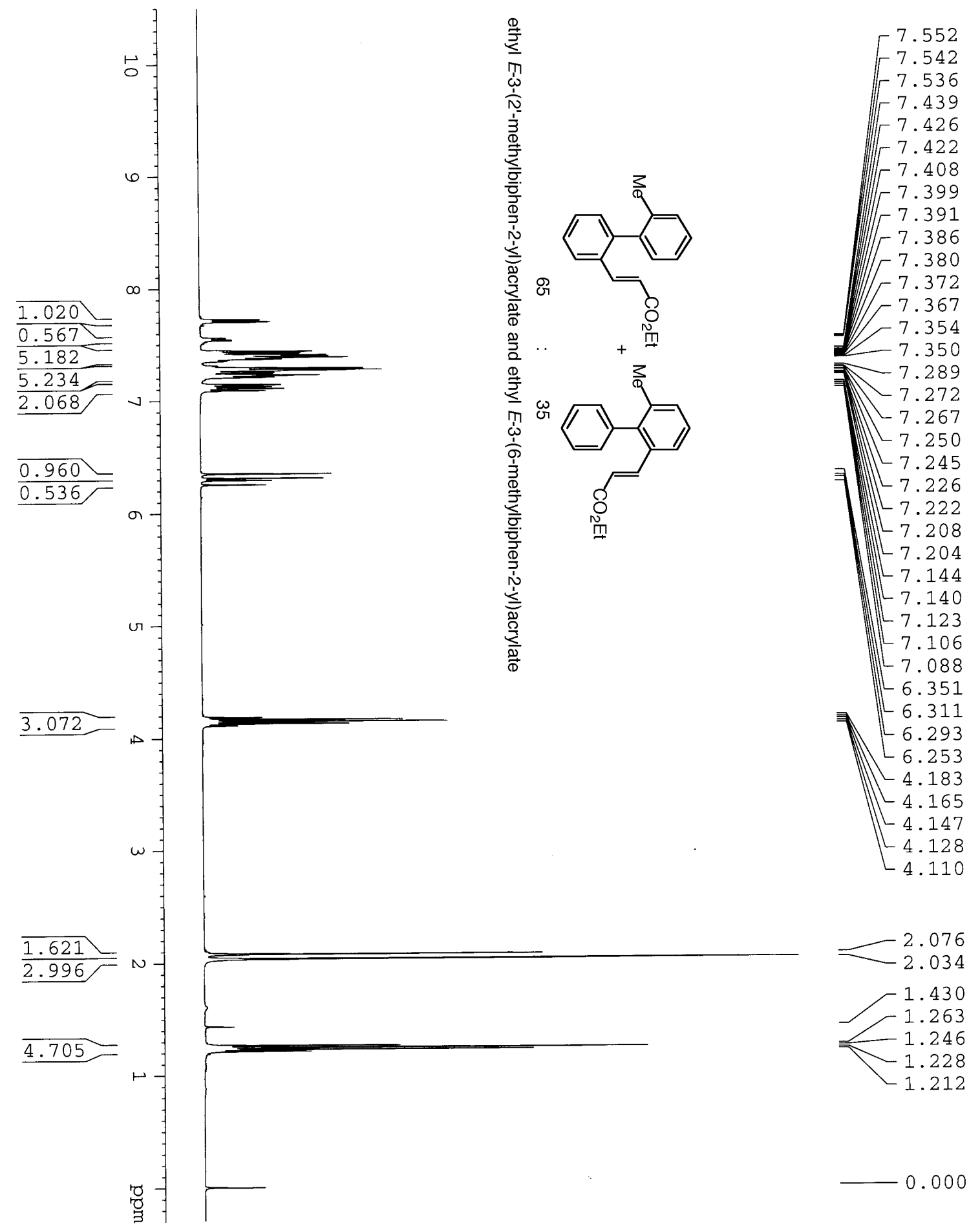

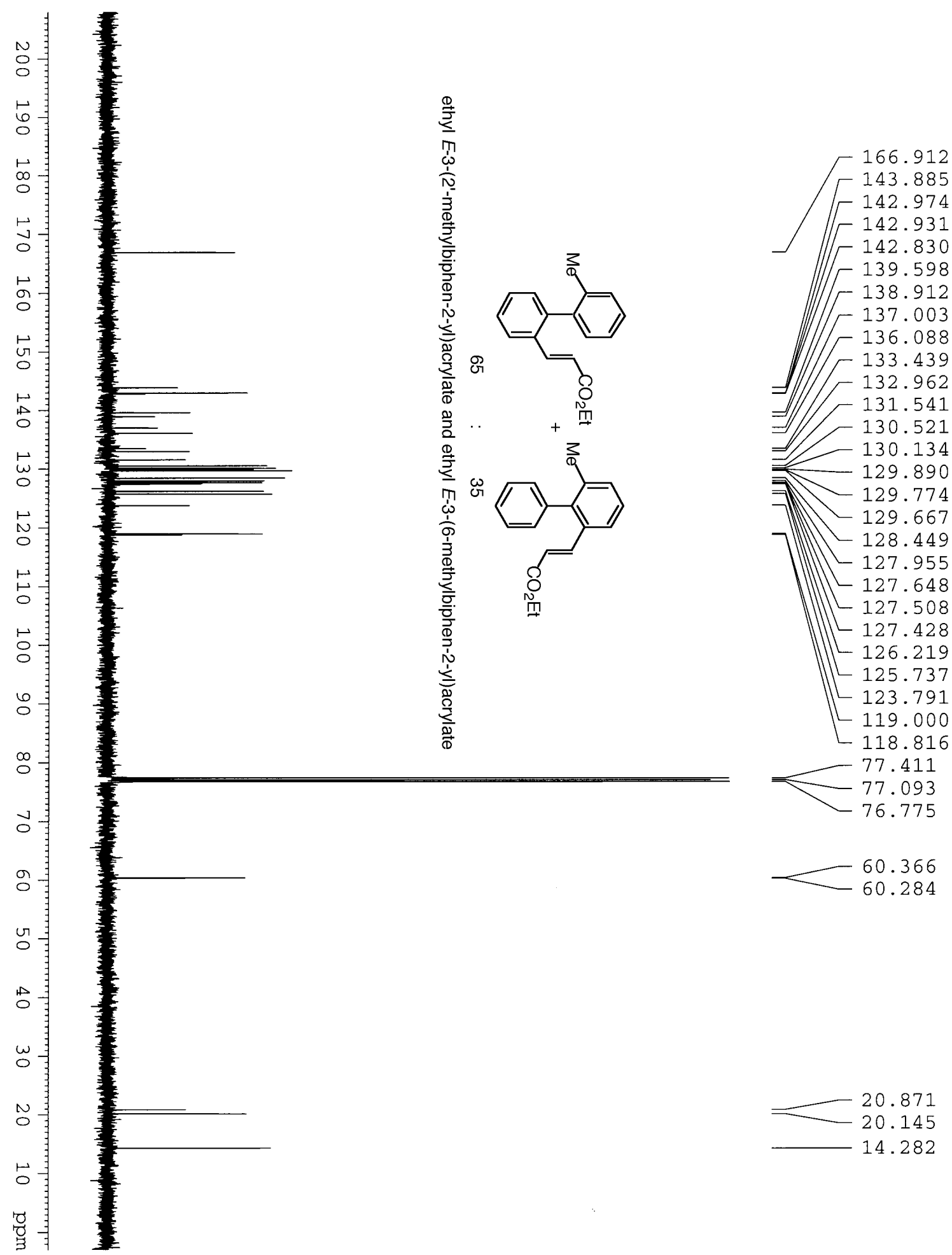

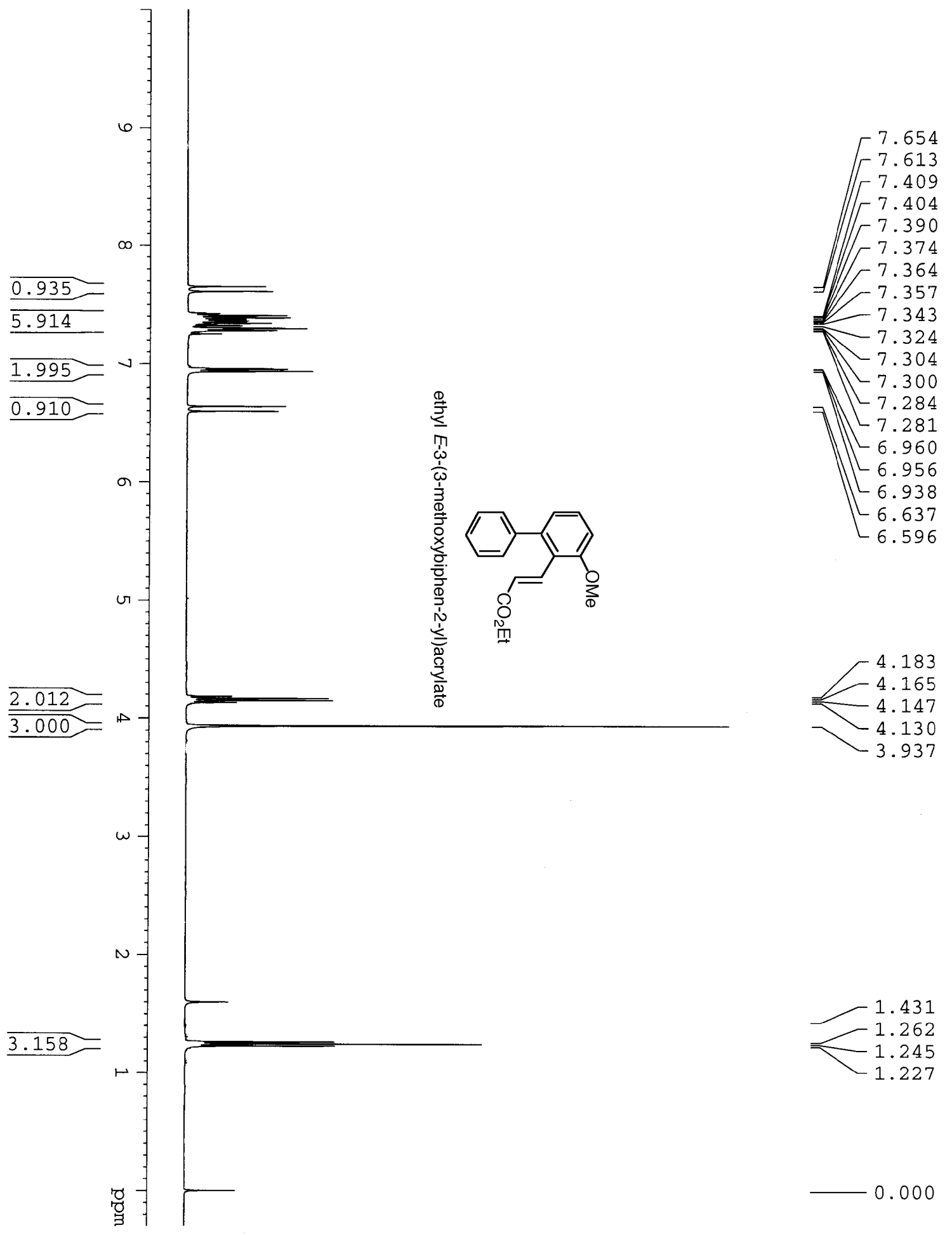

0.000 


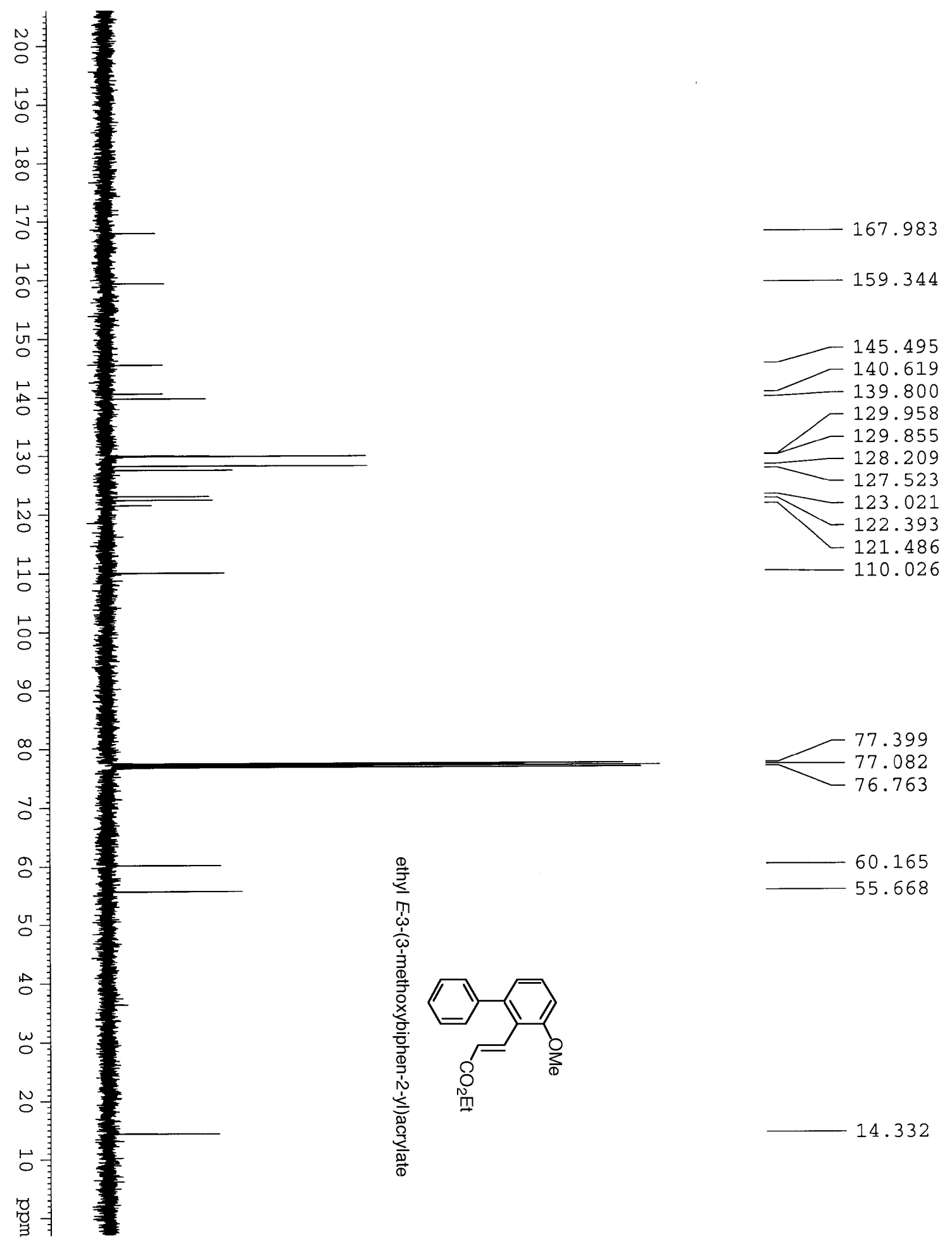



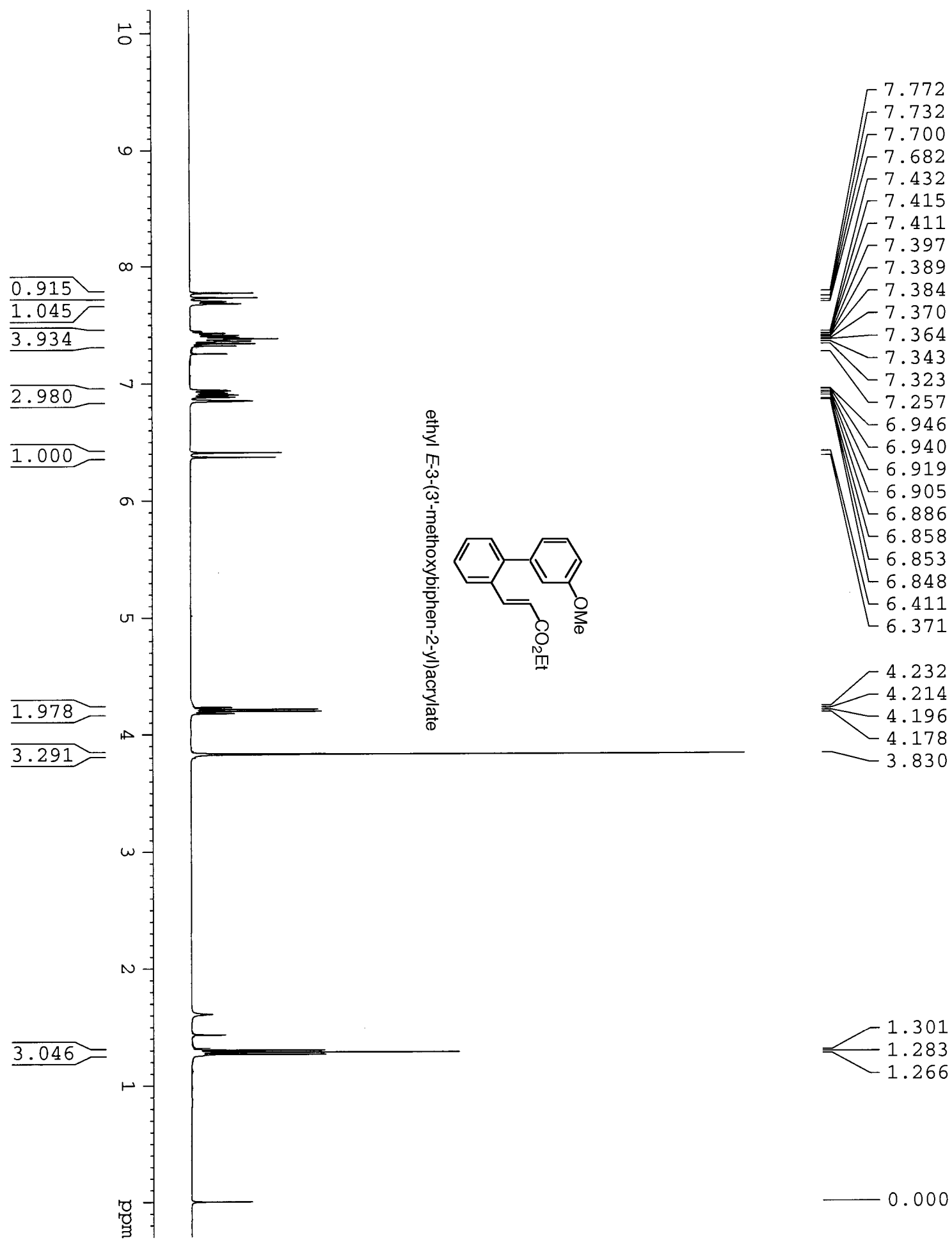

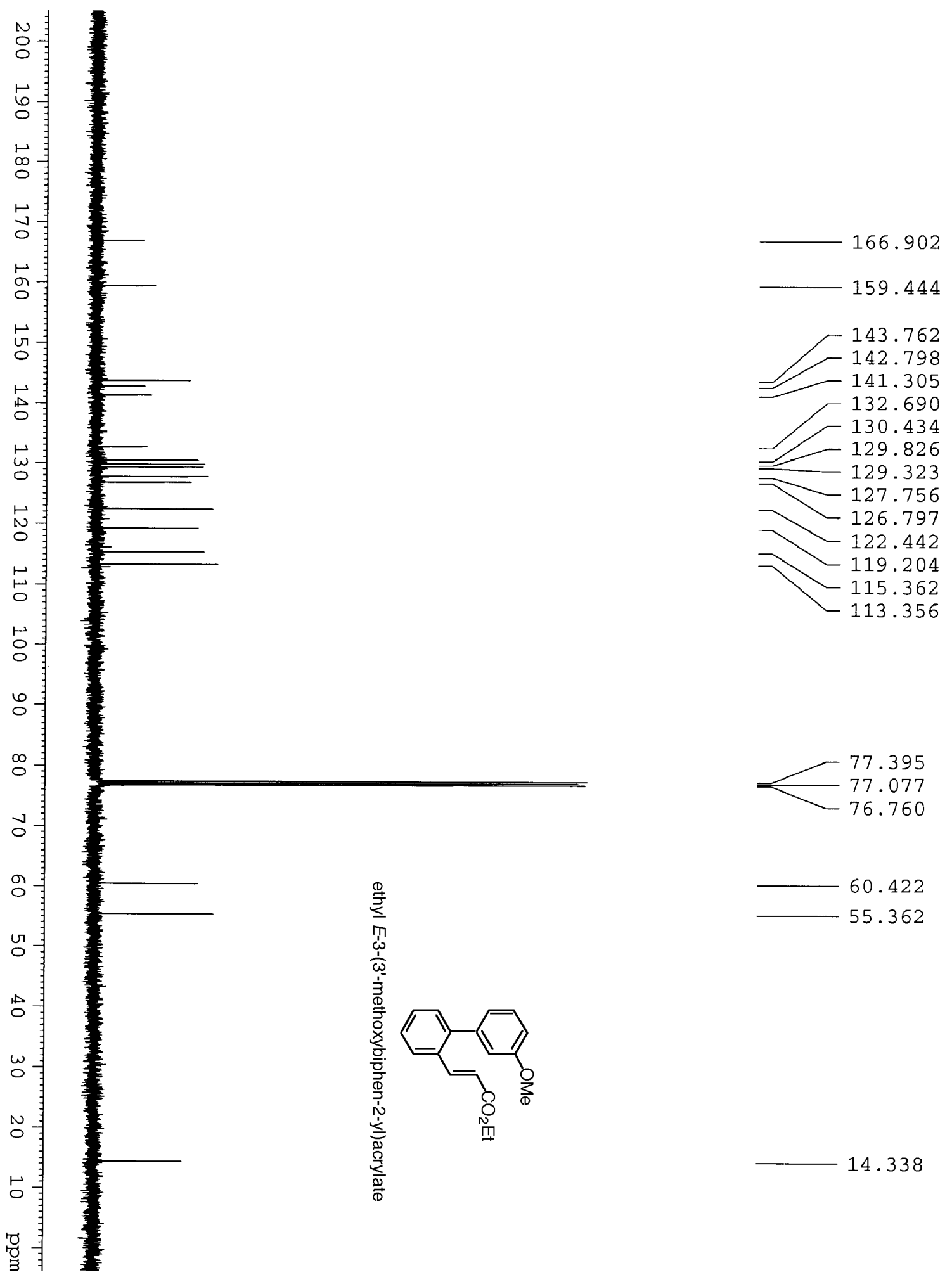

60.422

55.362

14.338 

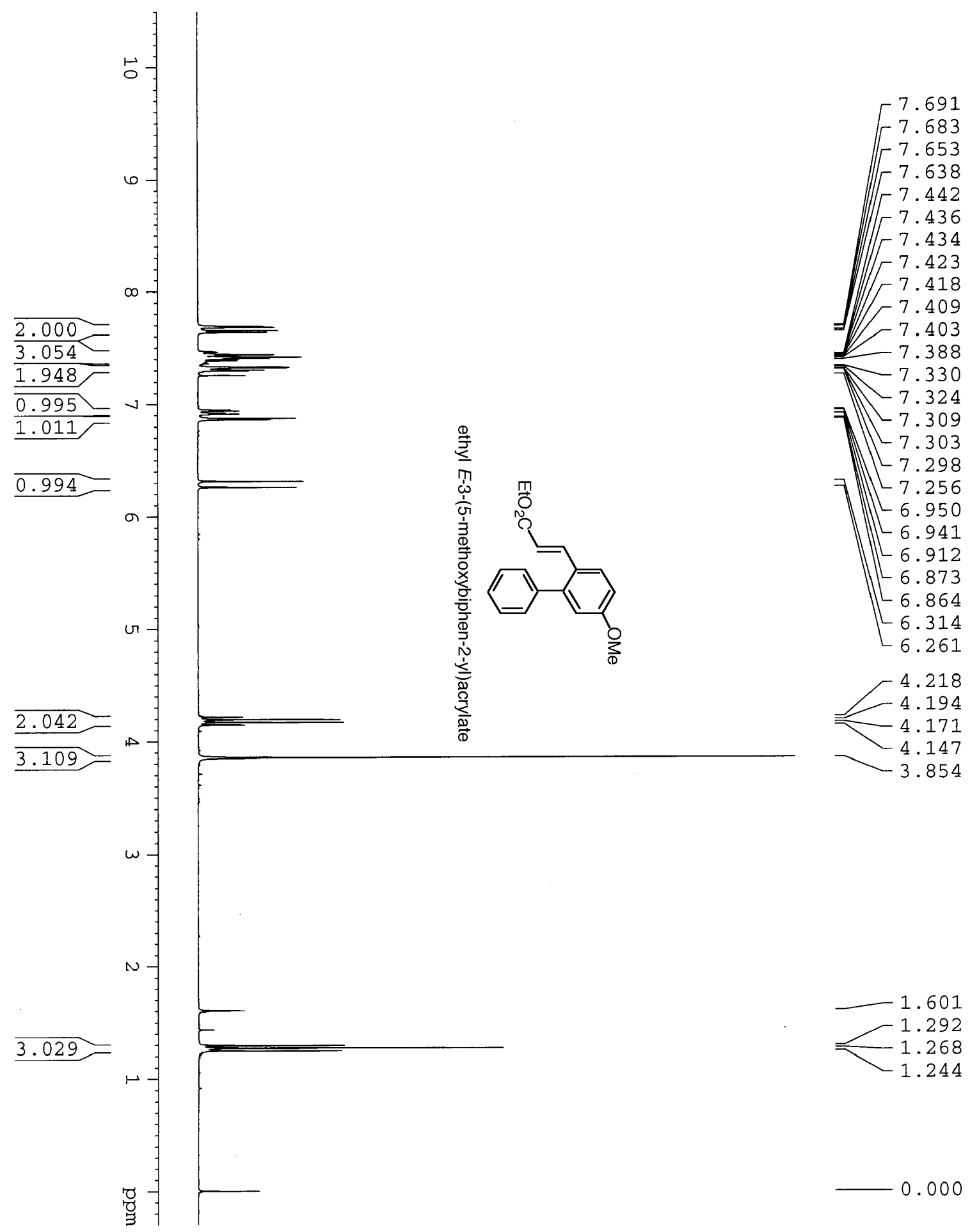


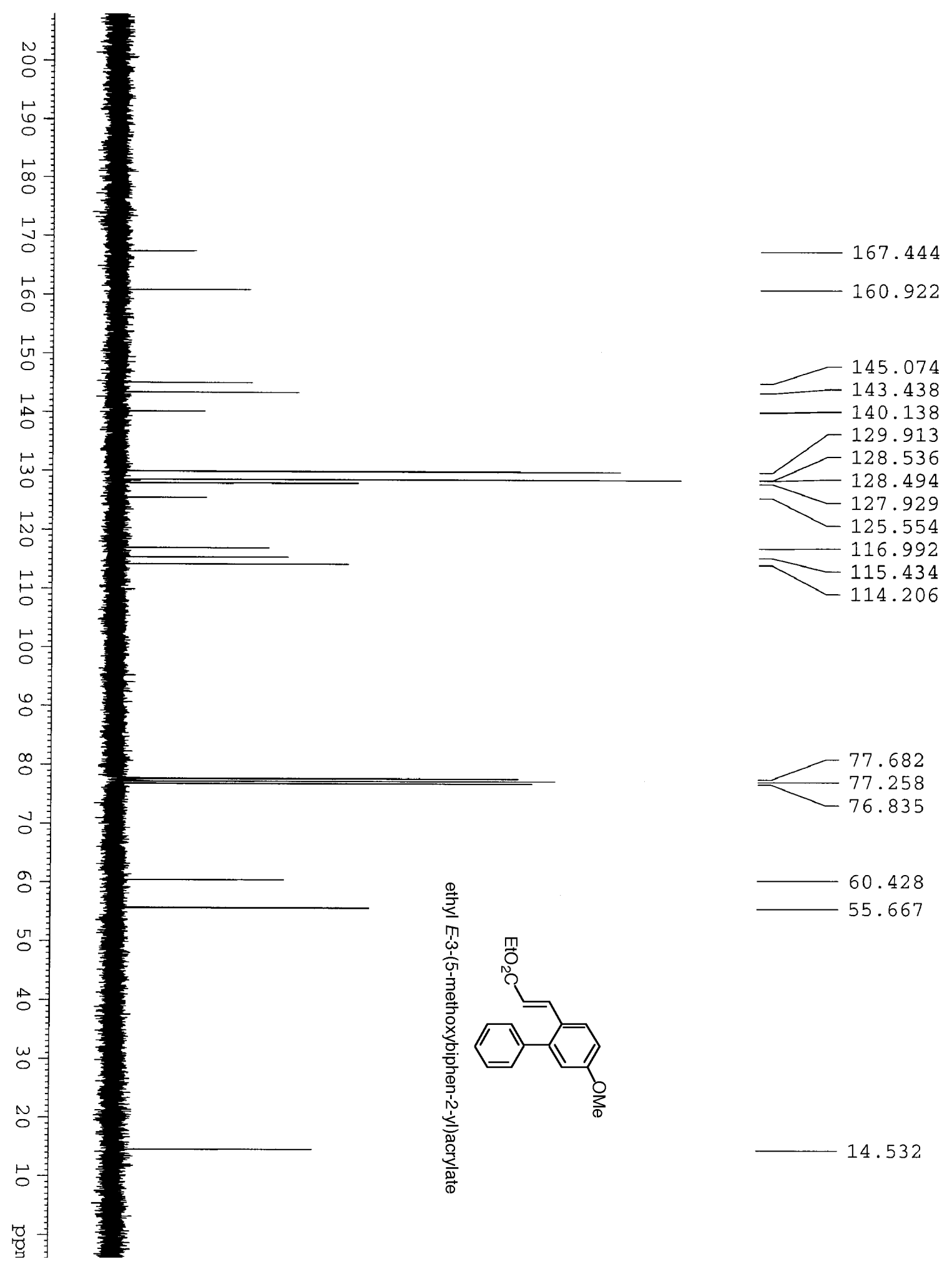



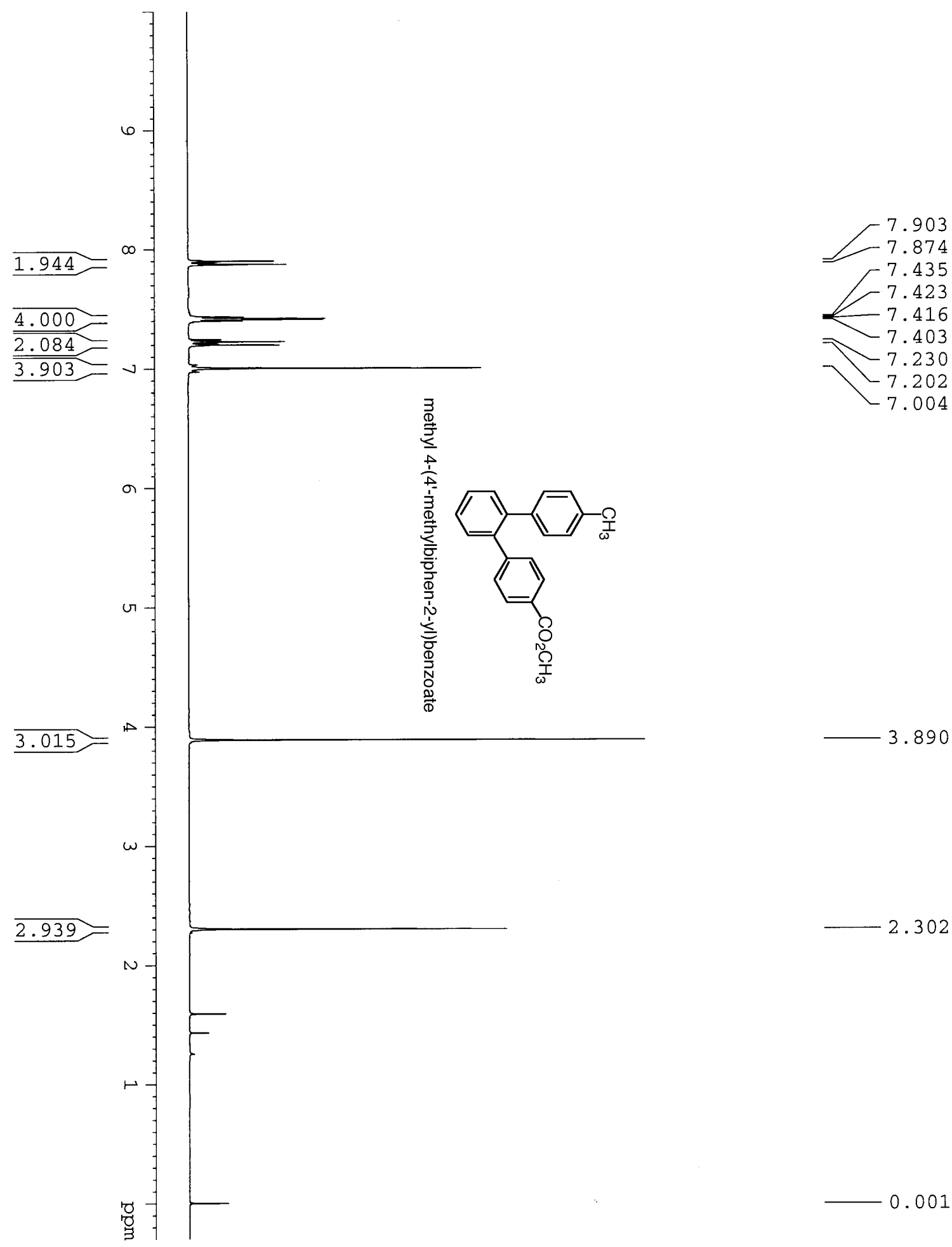

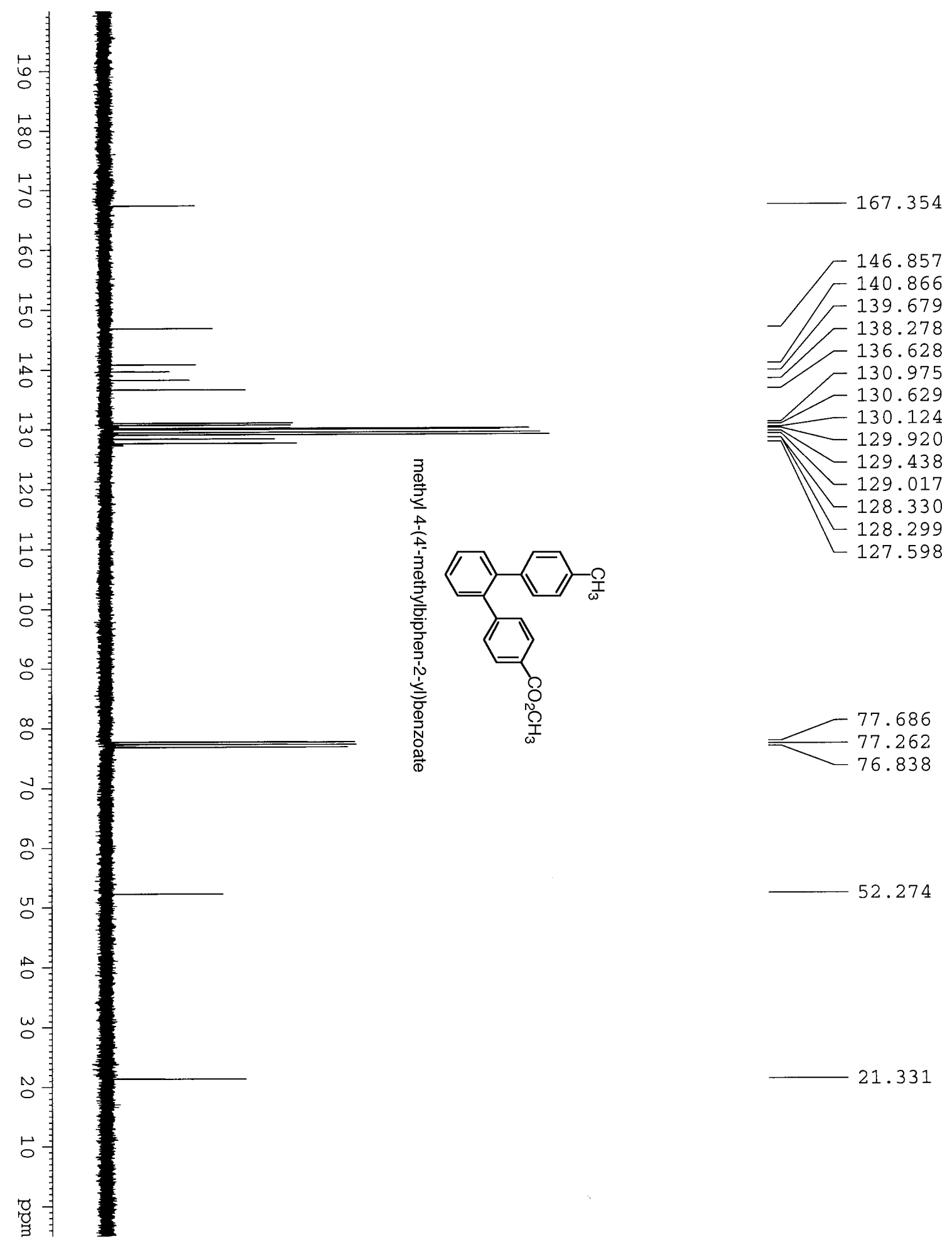

52.274

21.331 

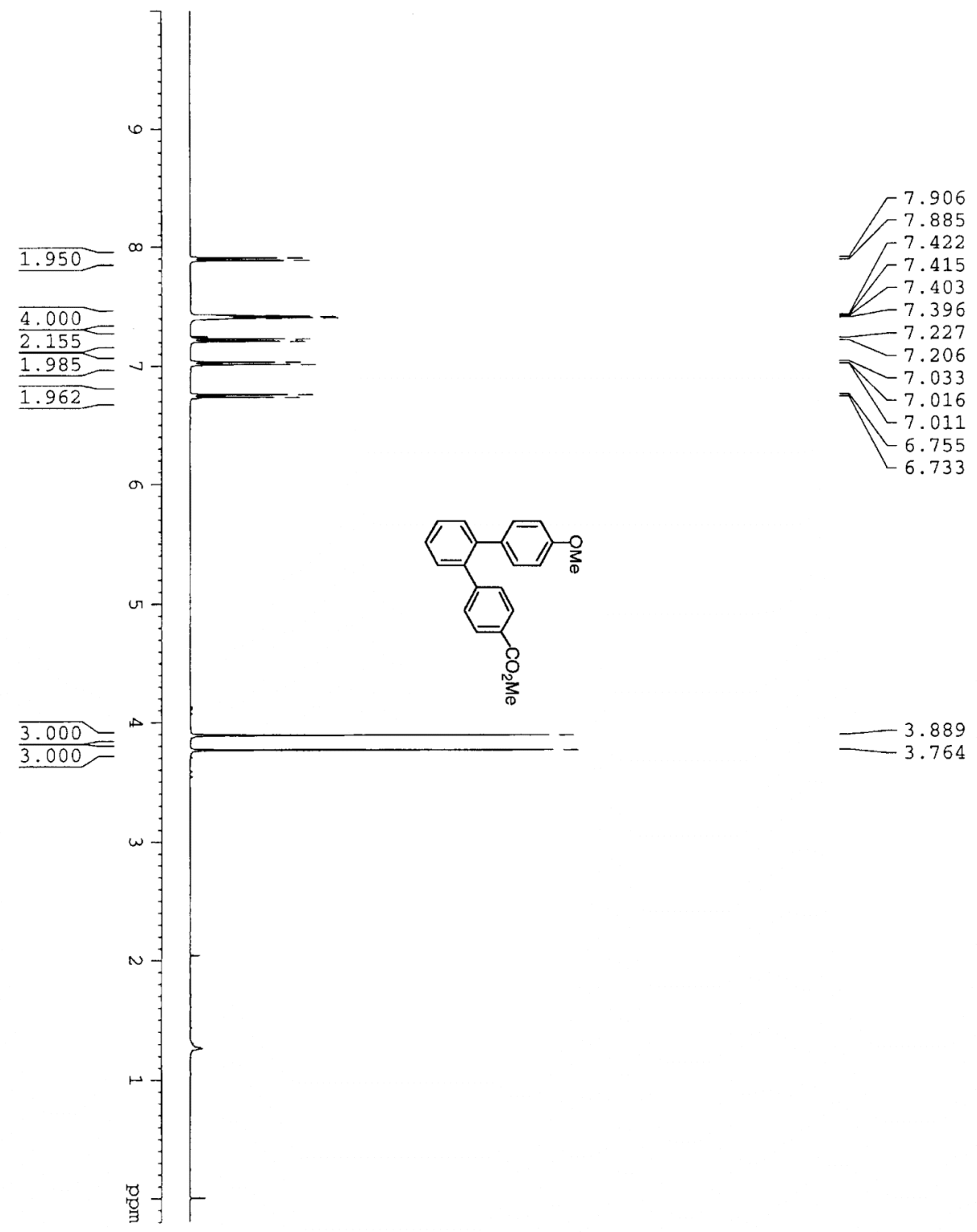
-3.889
$-\quad 3.764$ 

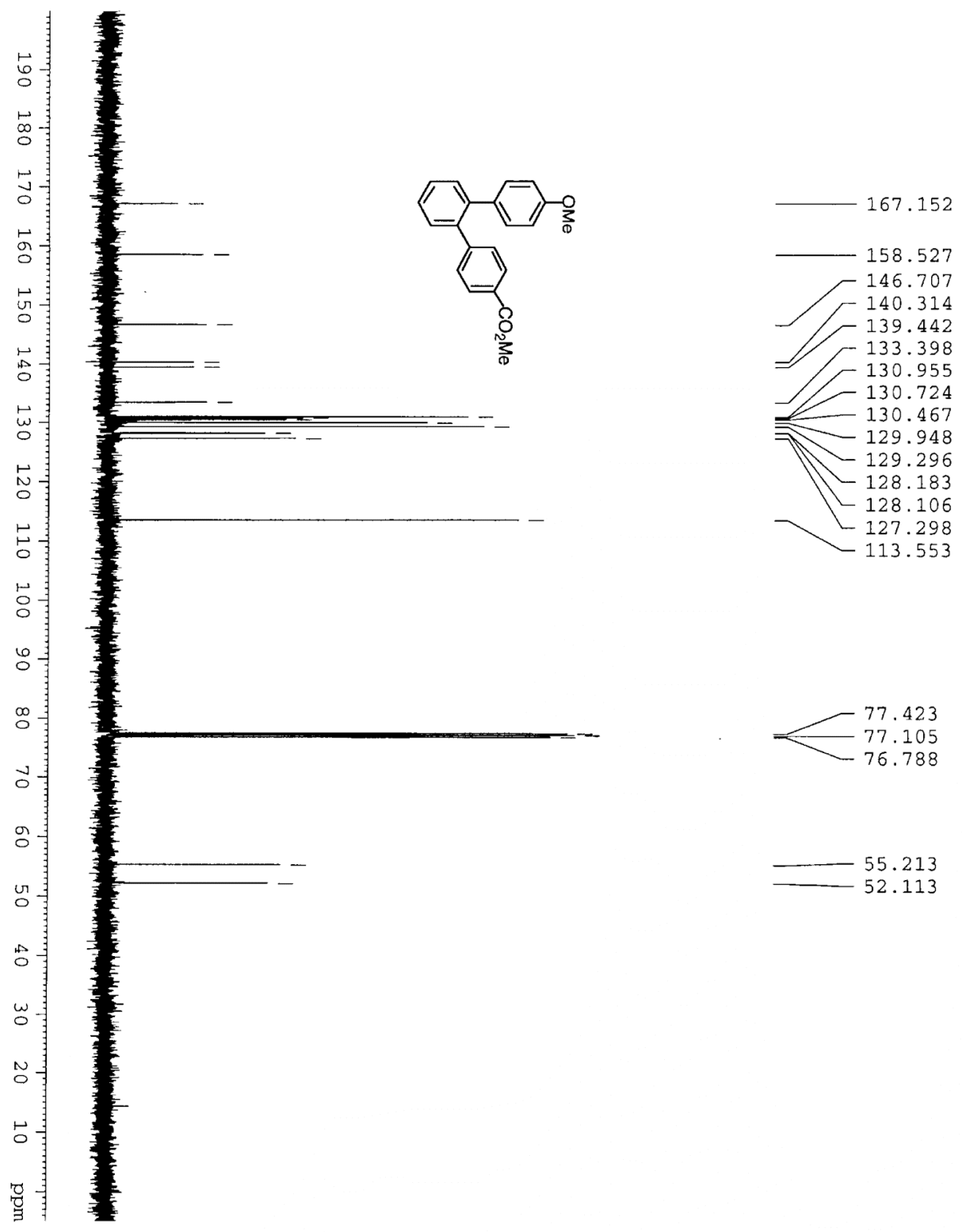

- 55.213

$-52.113$ 

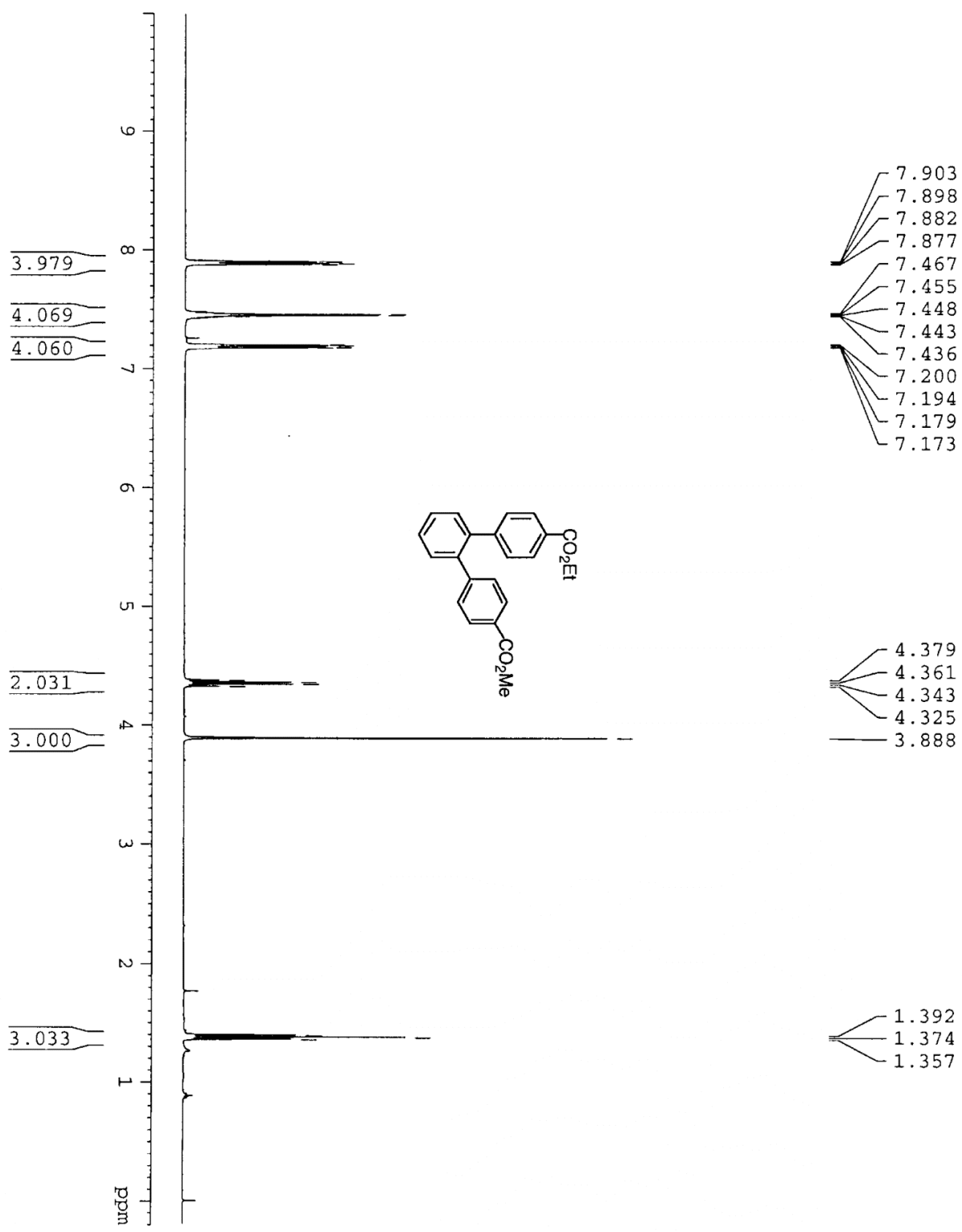

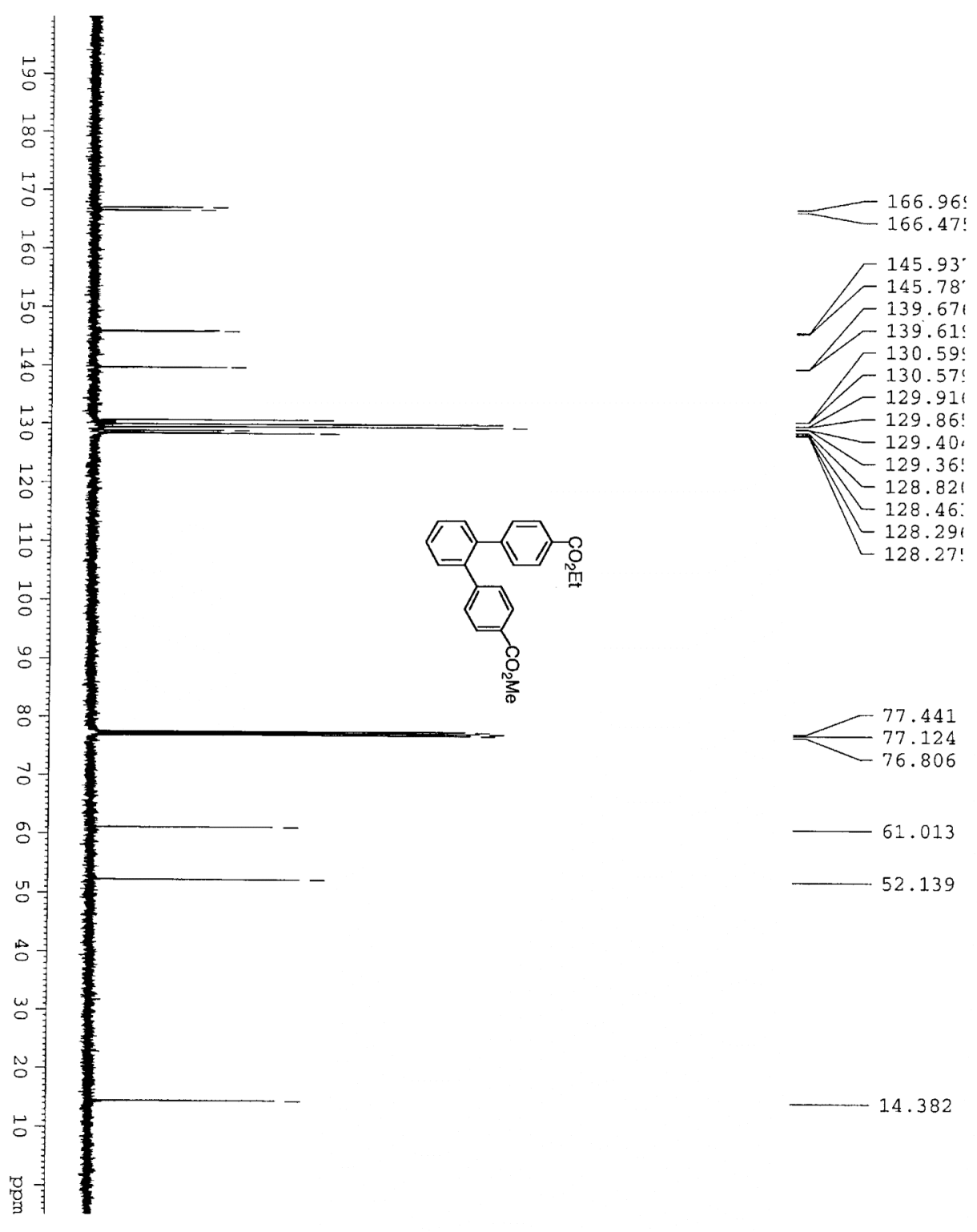

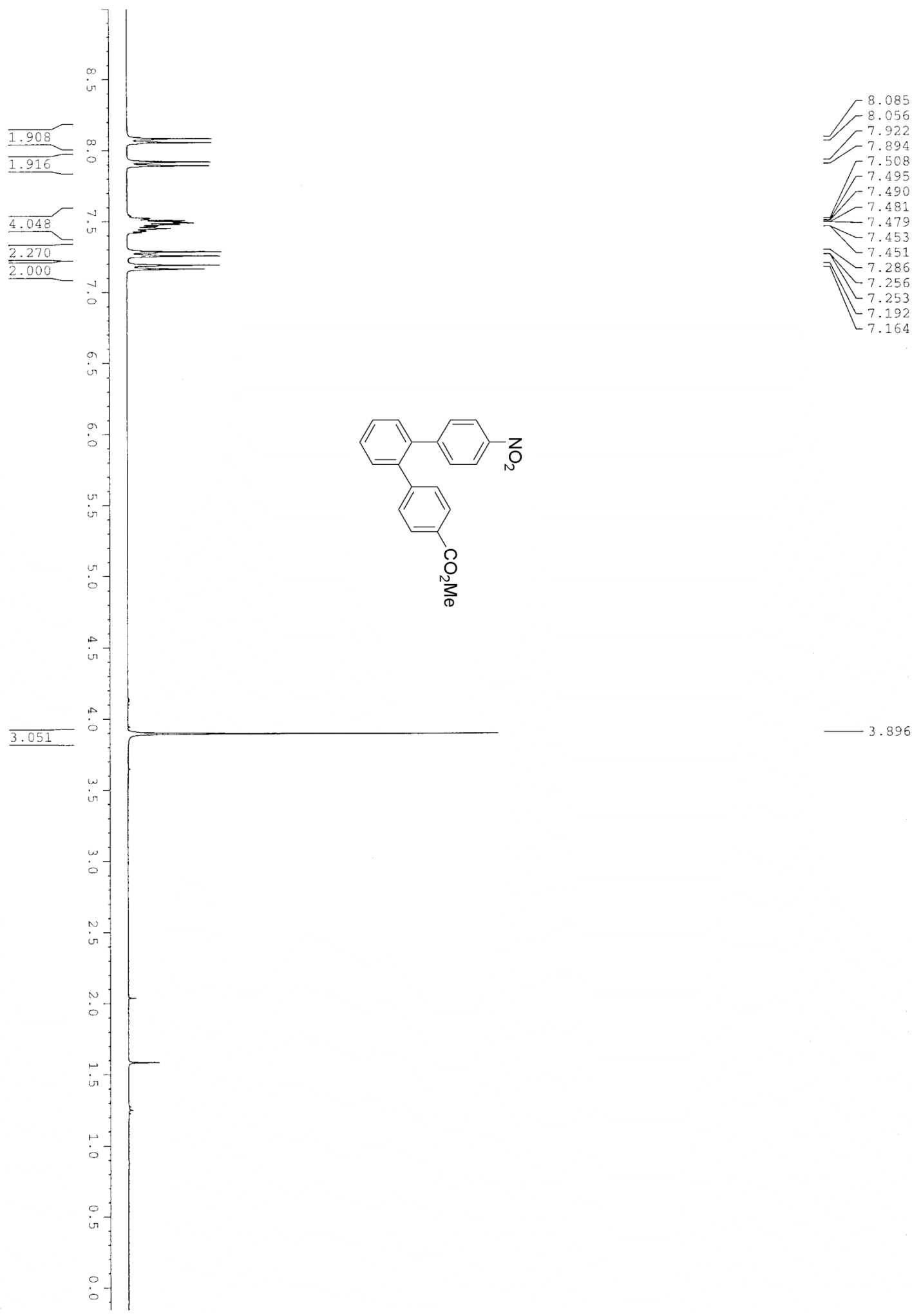

$-3.896$ 


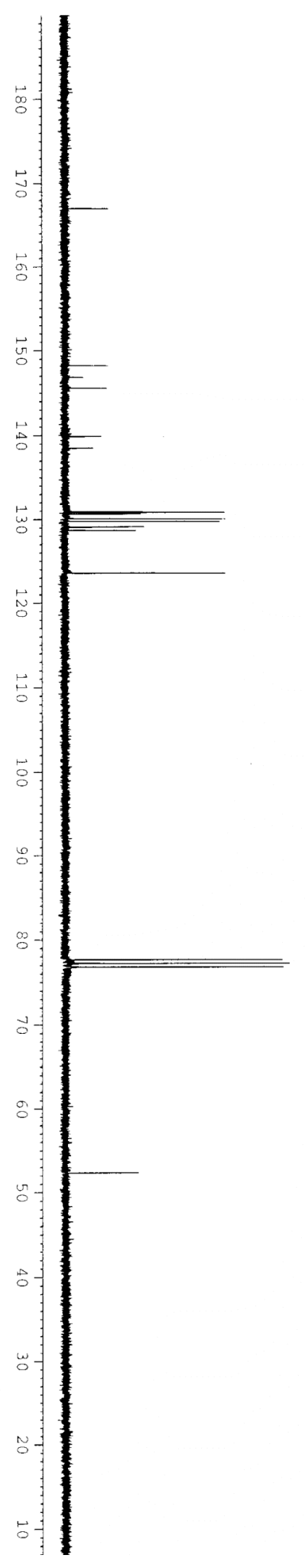

$-166.975$
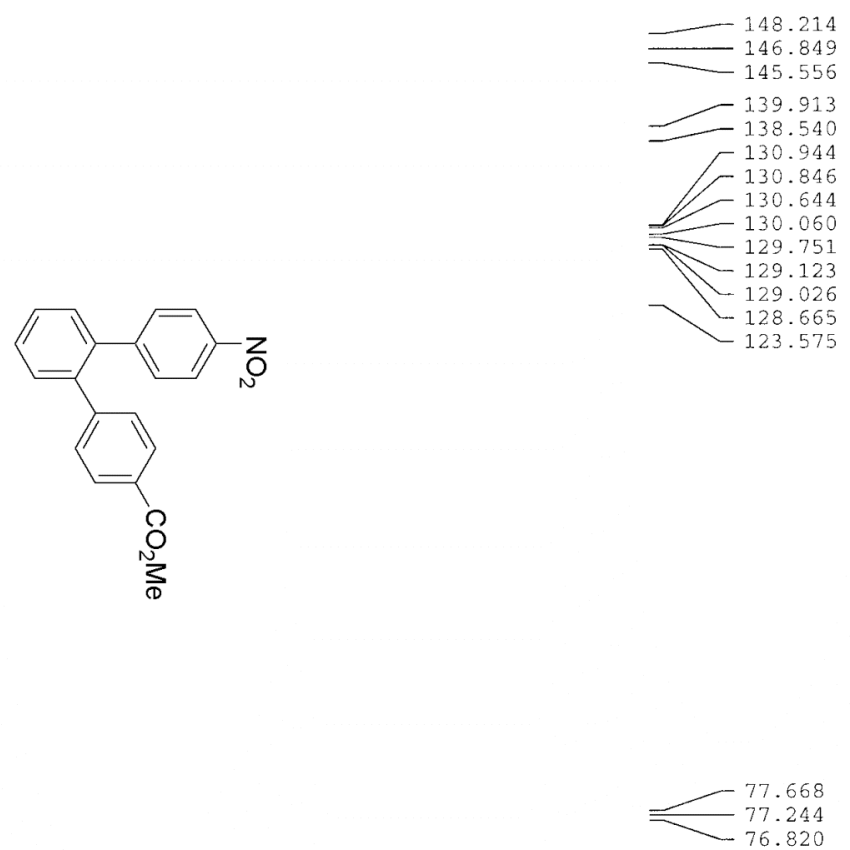

52.382 

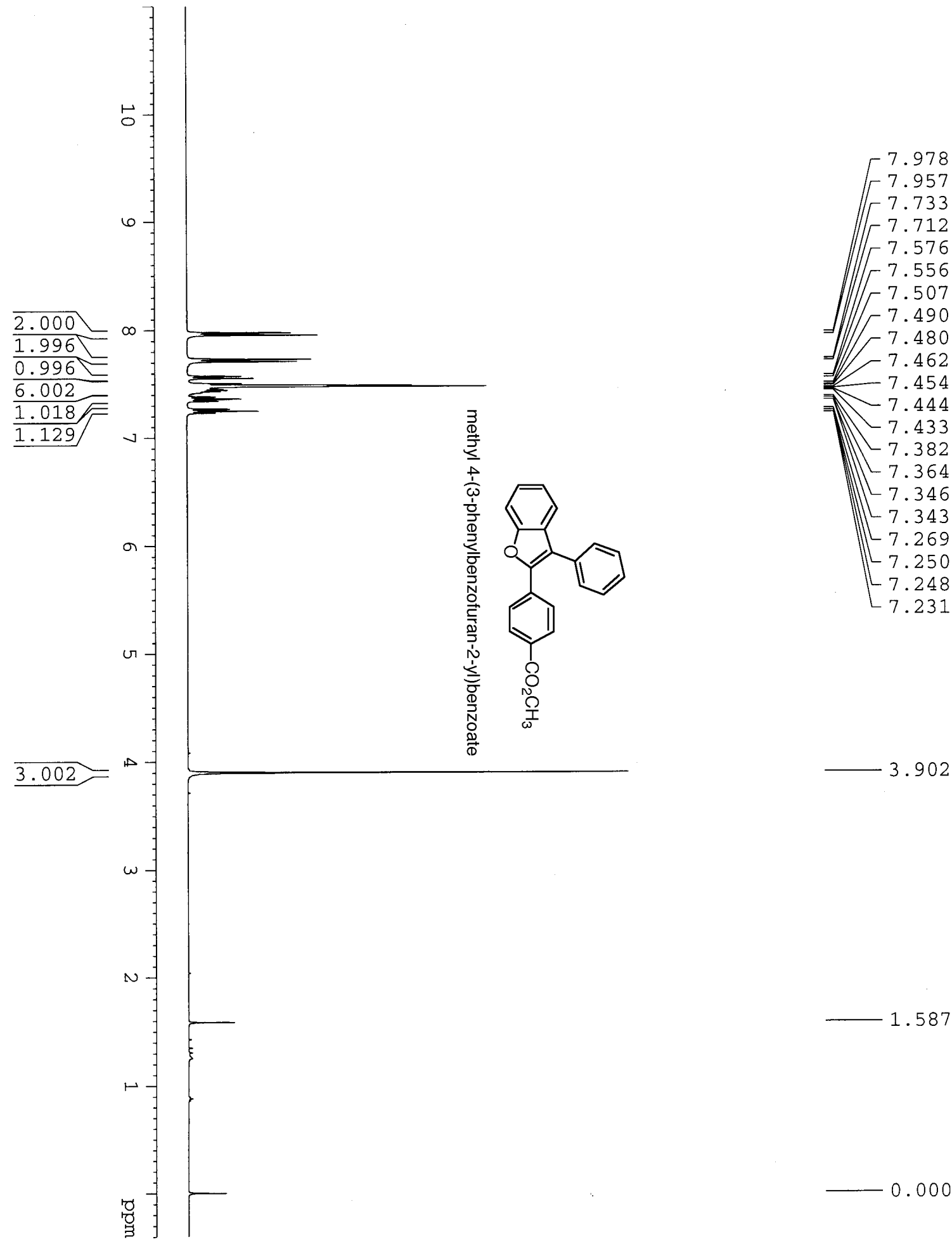

$-0.000$ 


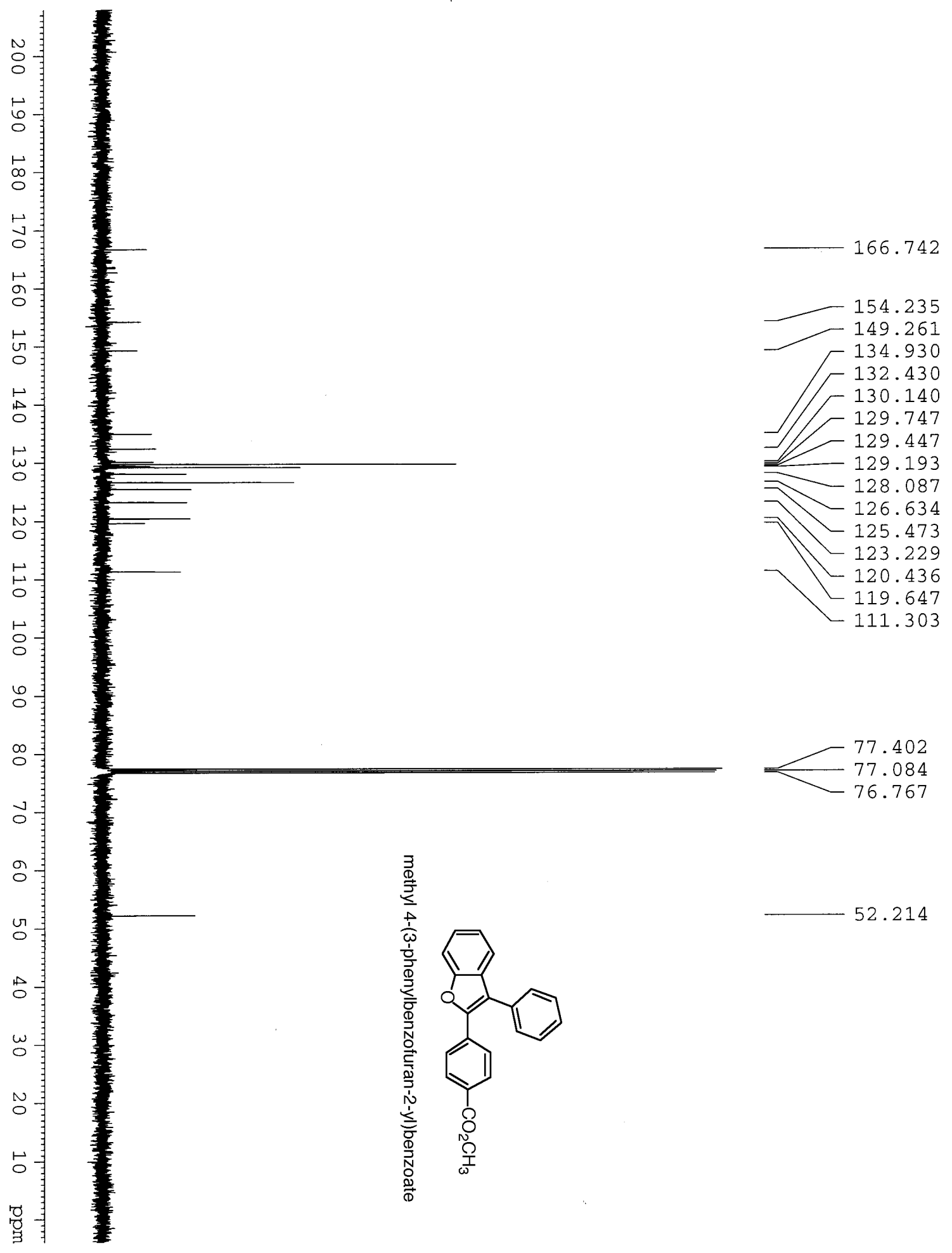



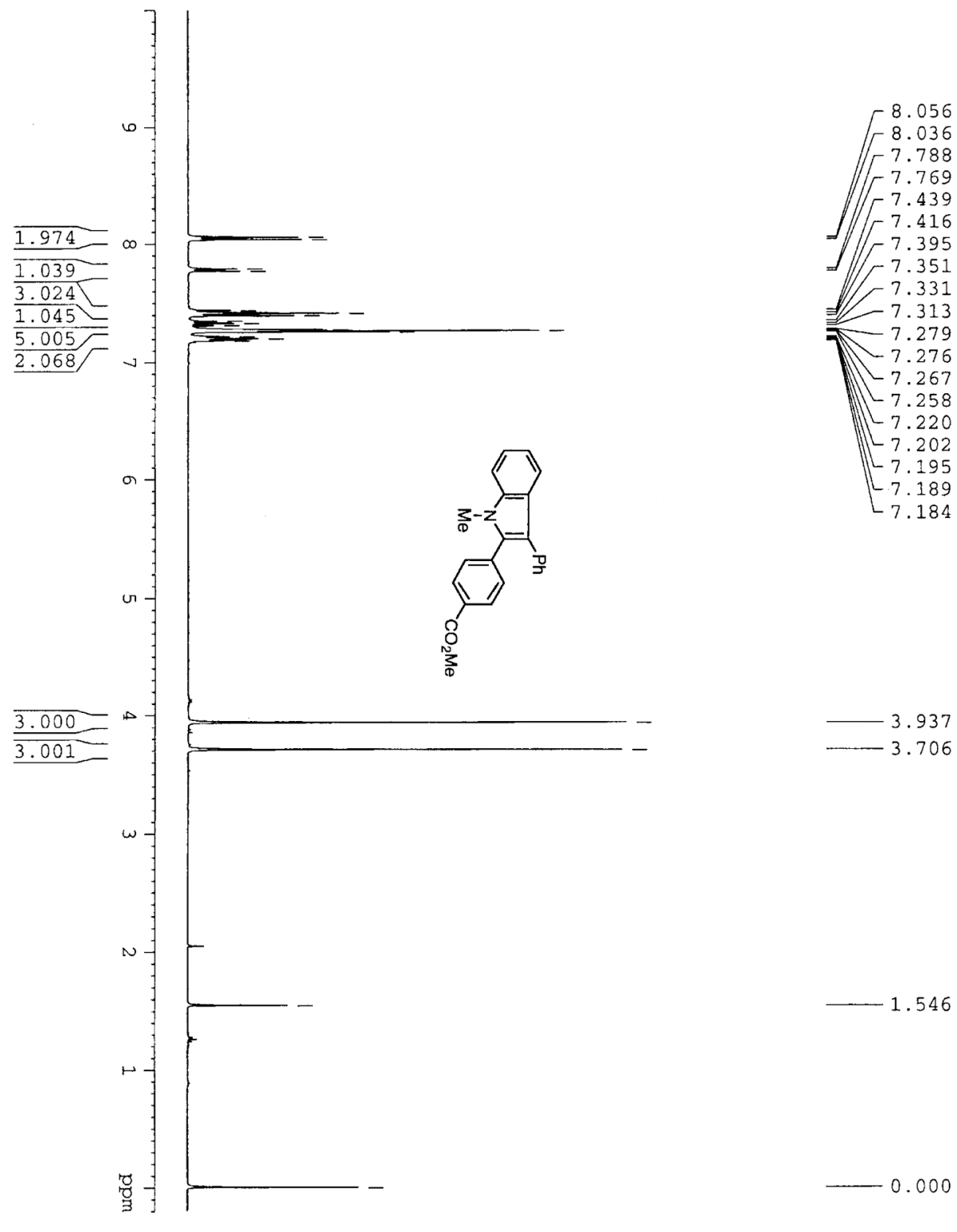

$-0.000$ 

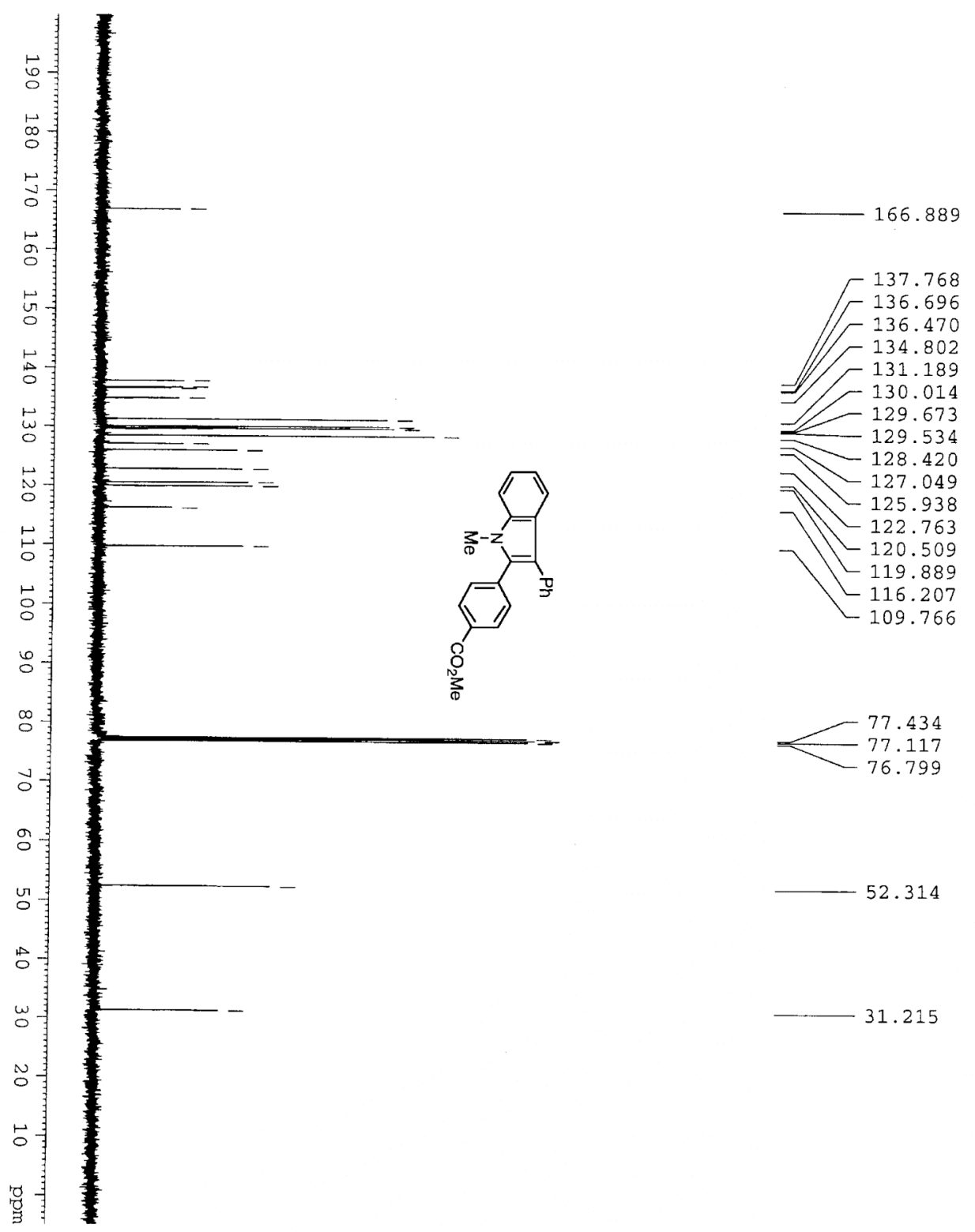

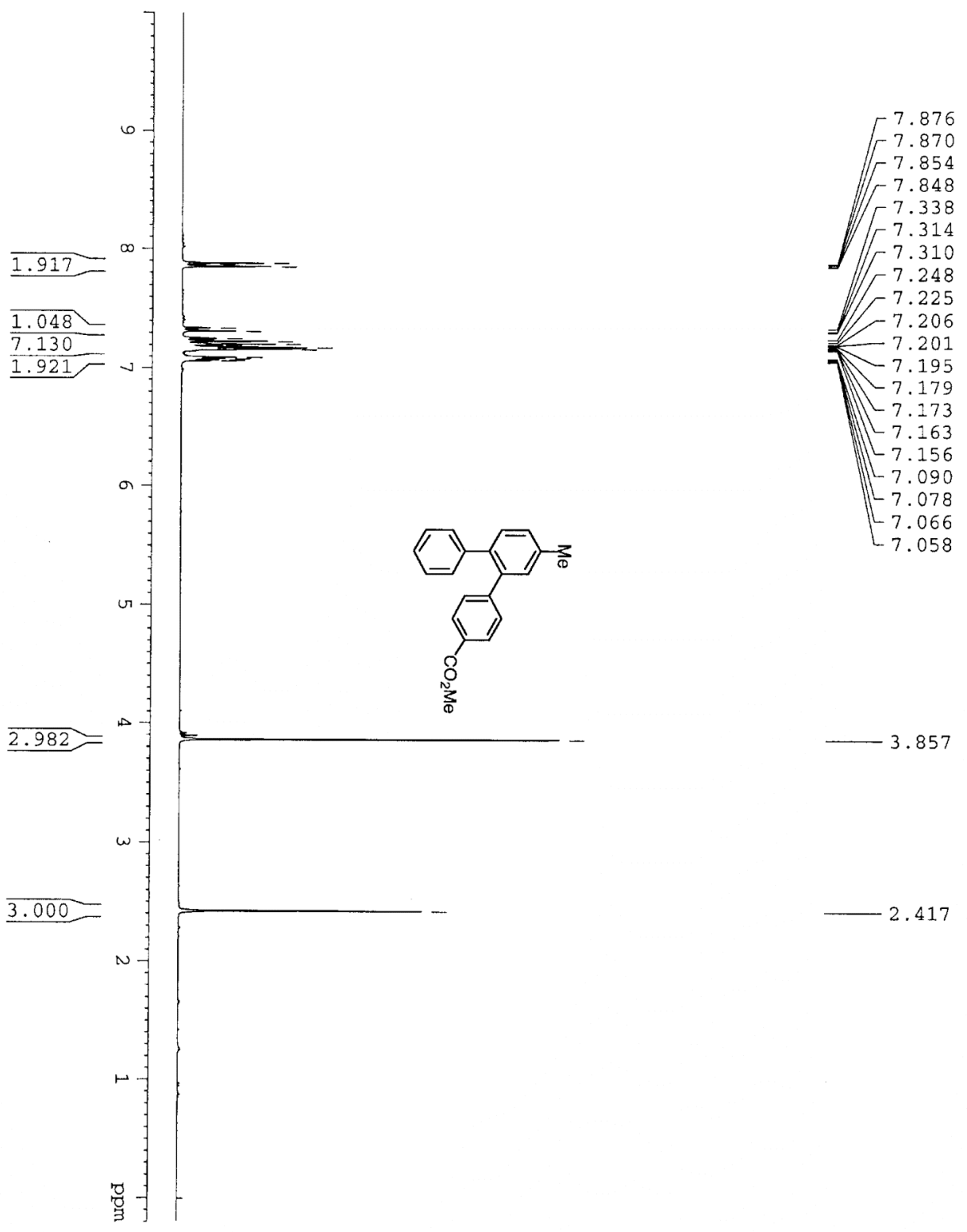

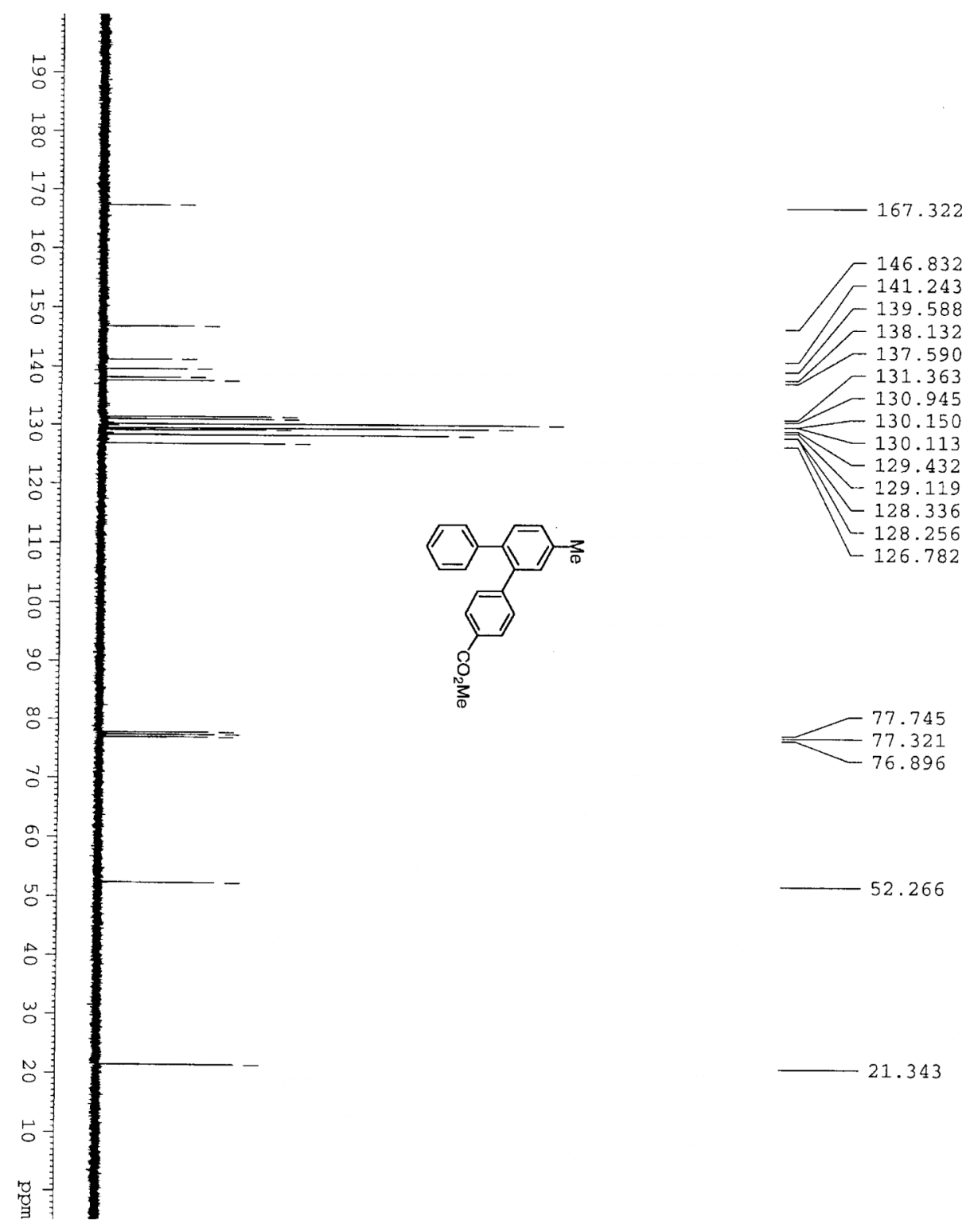

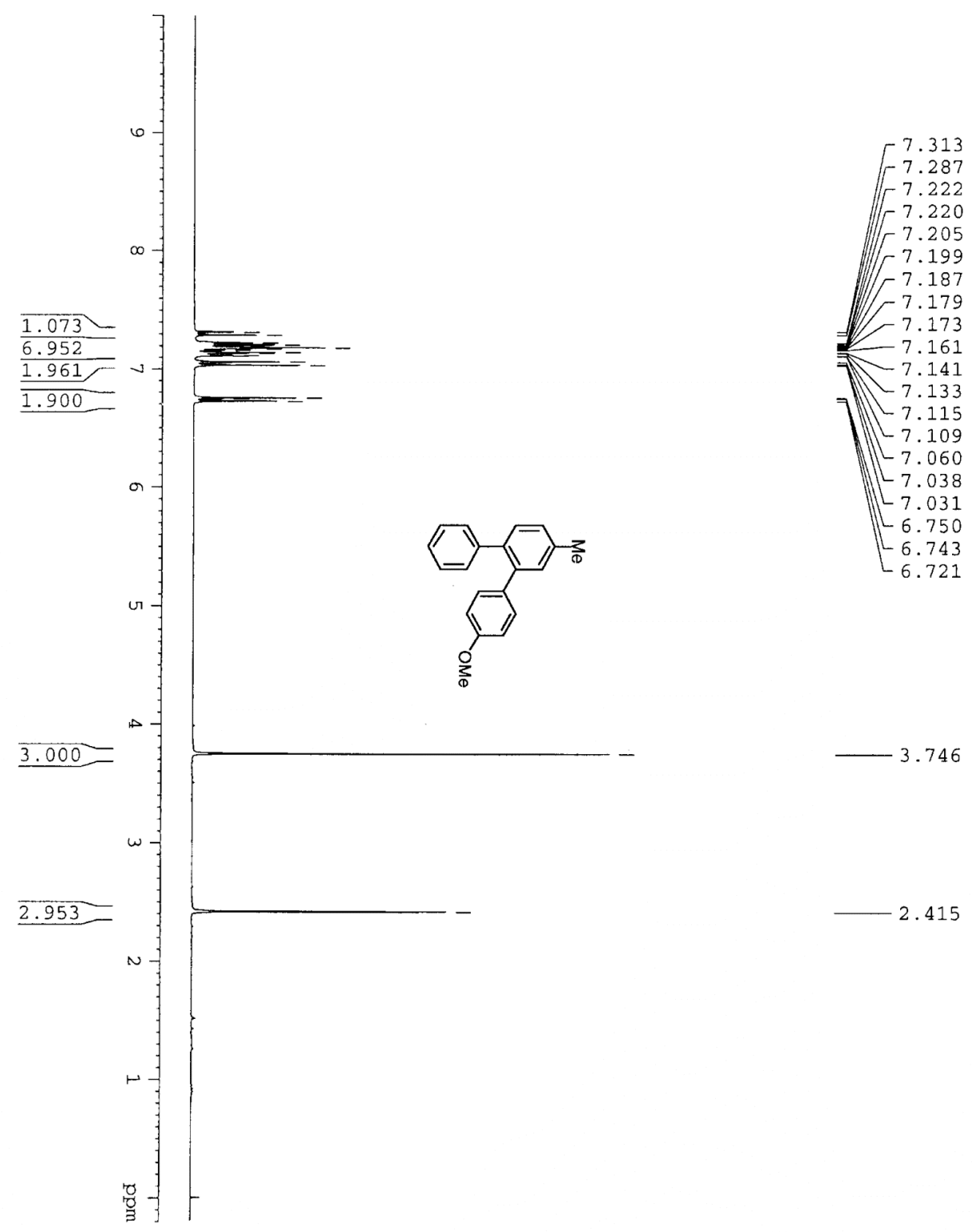

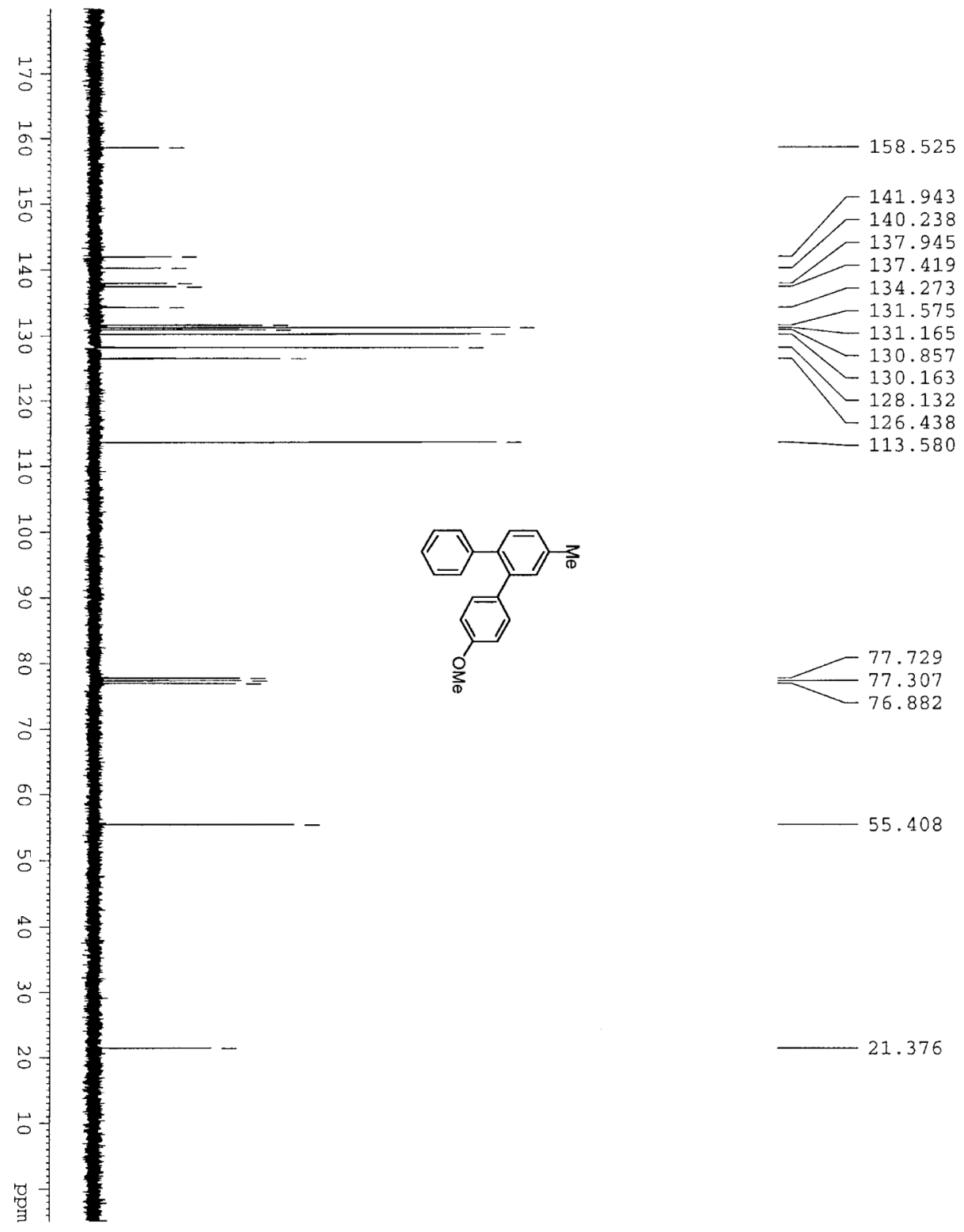

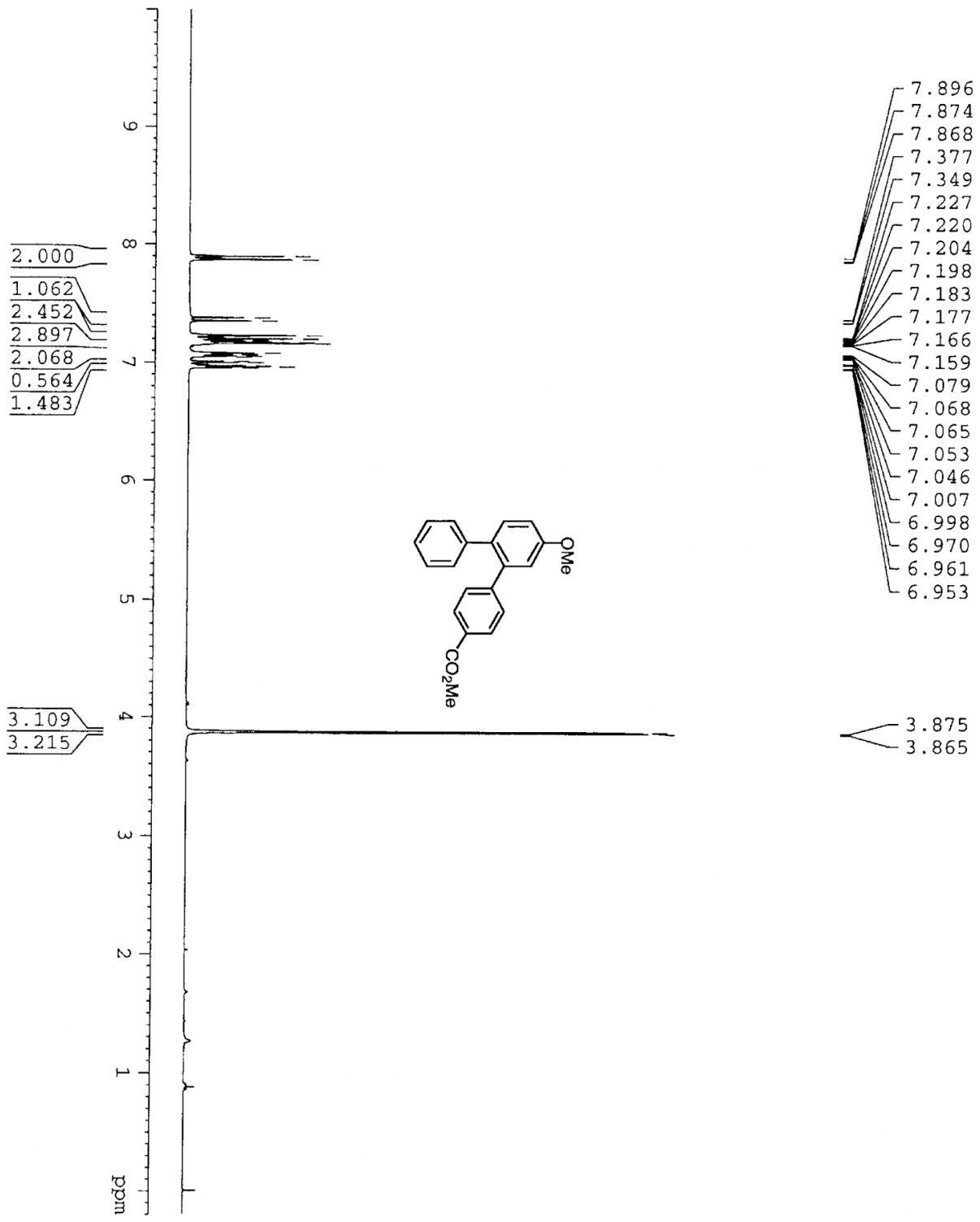

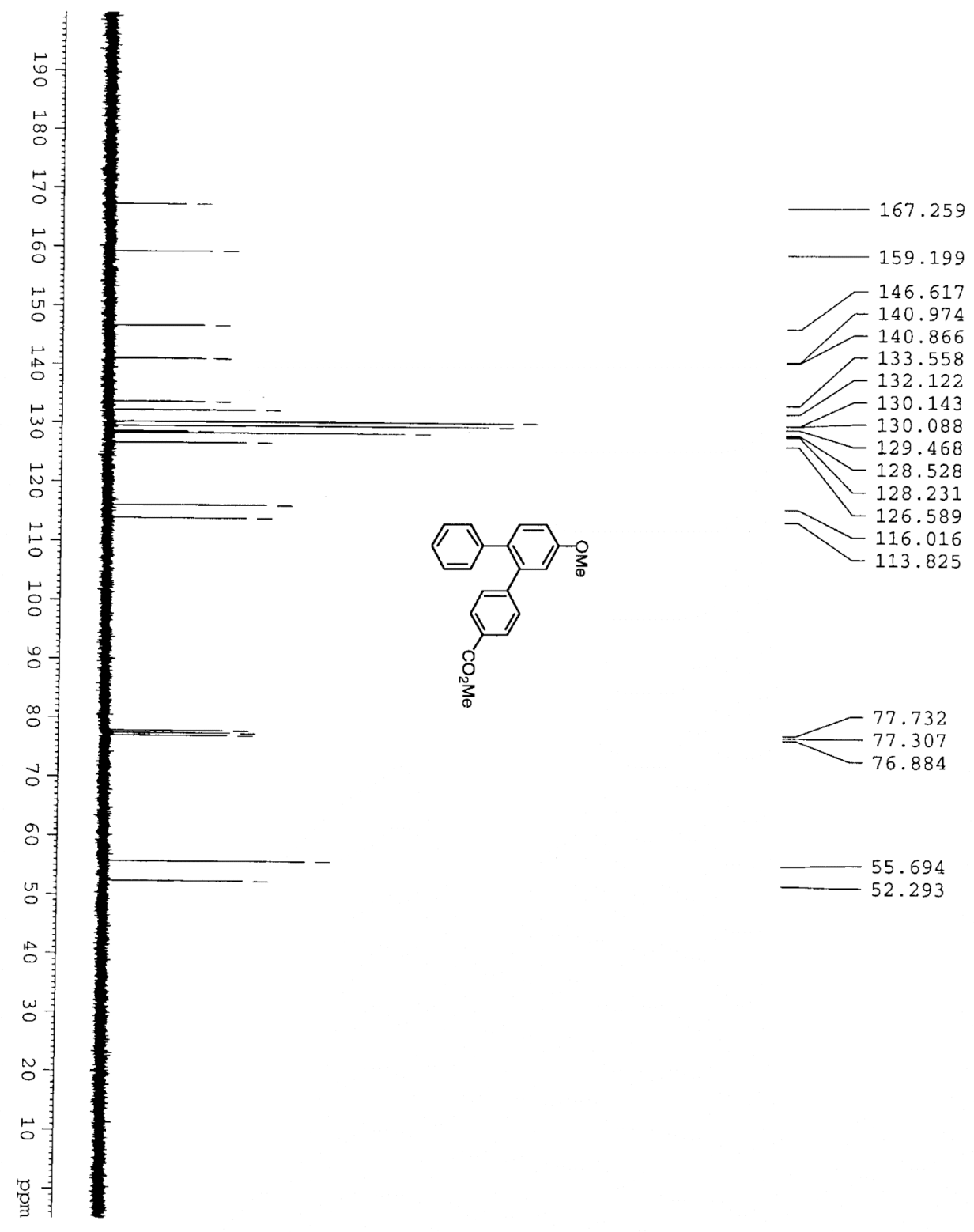

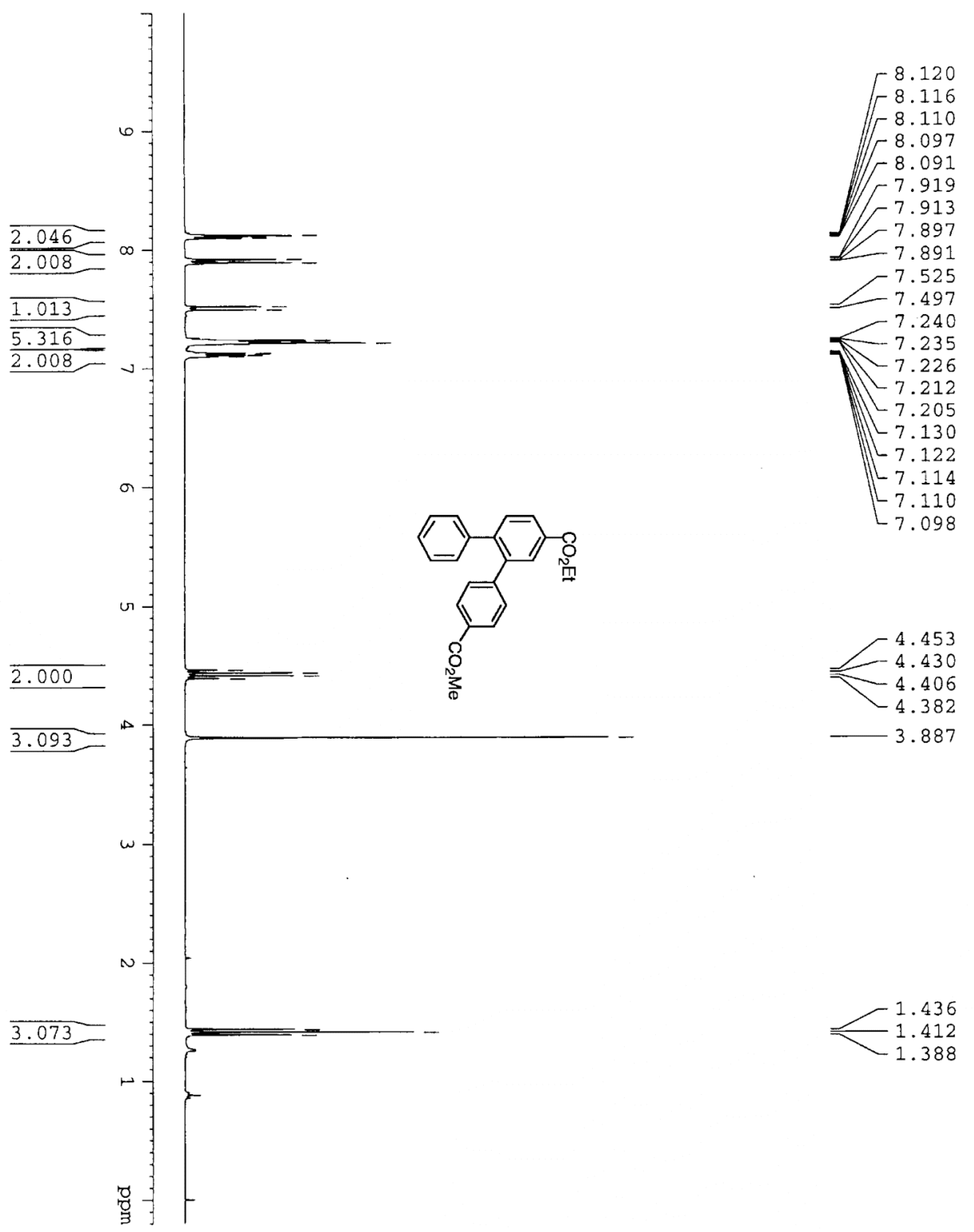

1.436

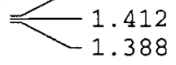



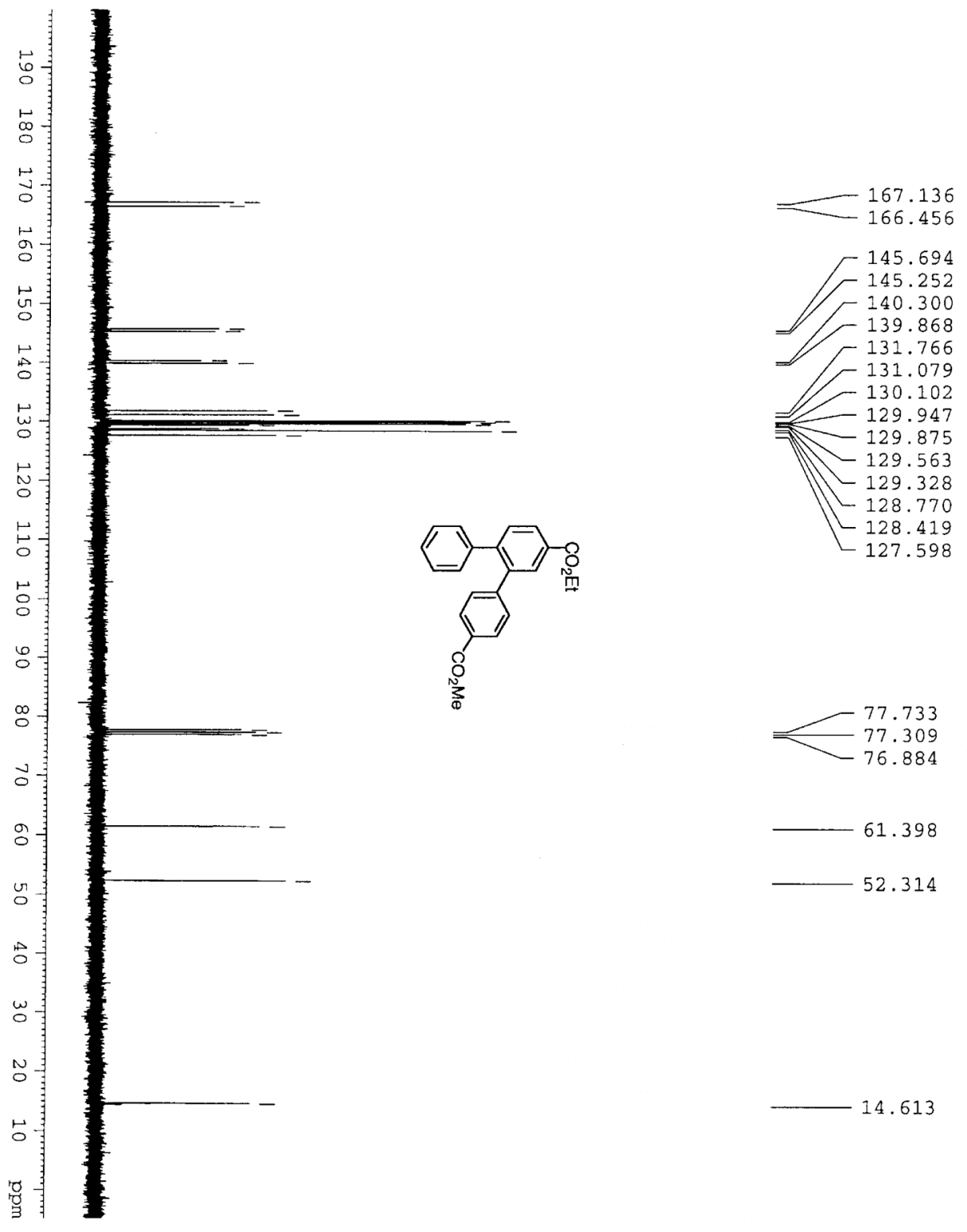

$-14.613$ 

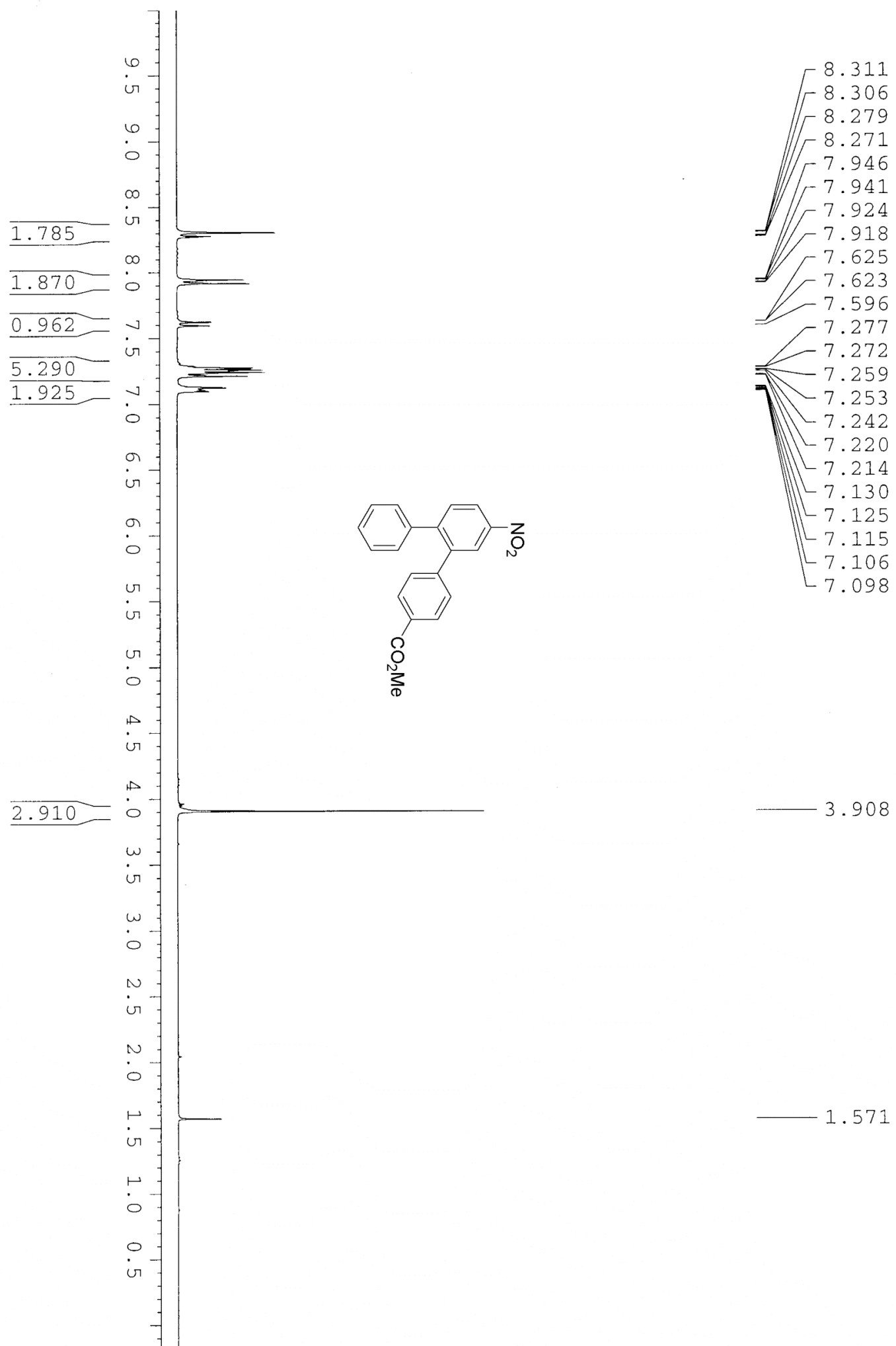

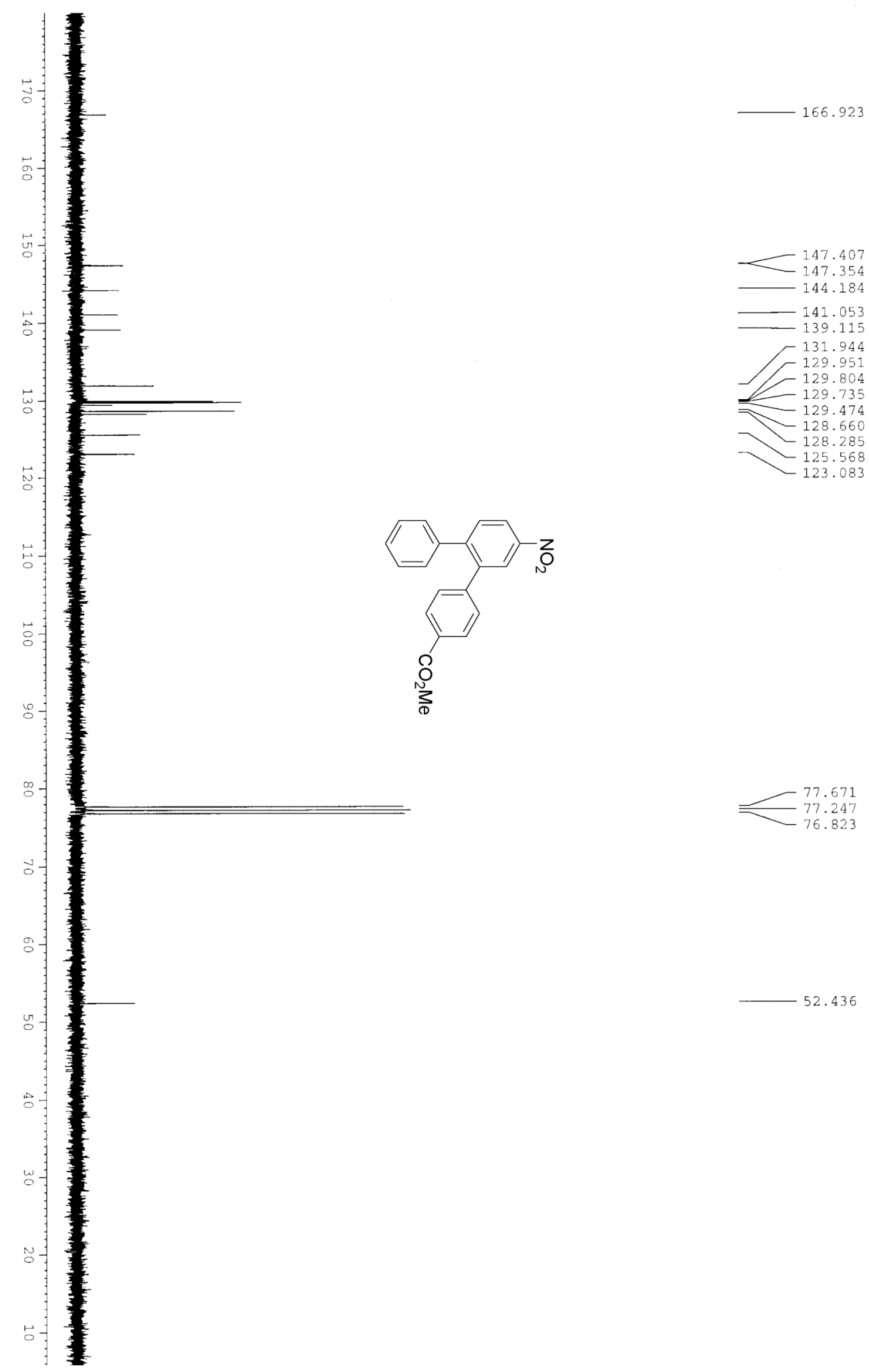

$-52.436$ 

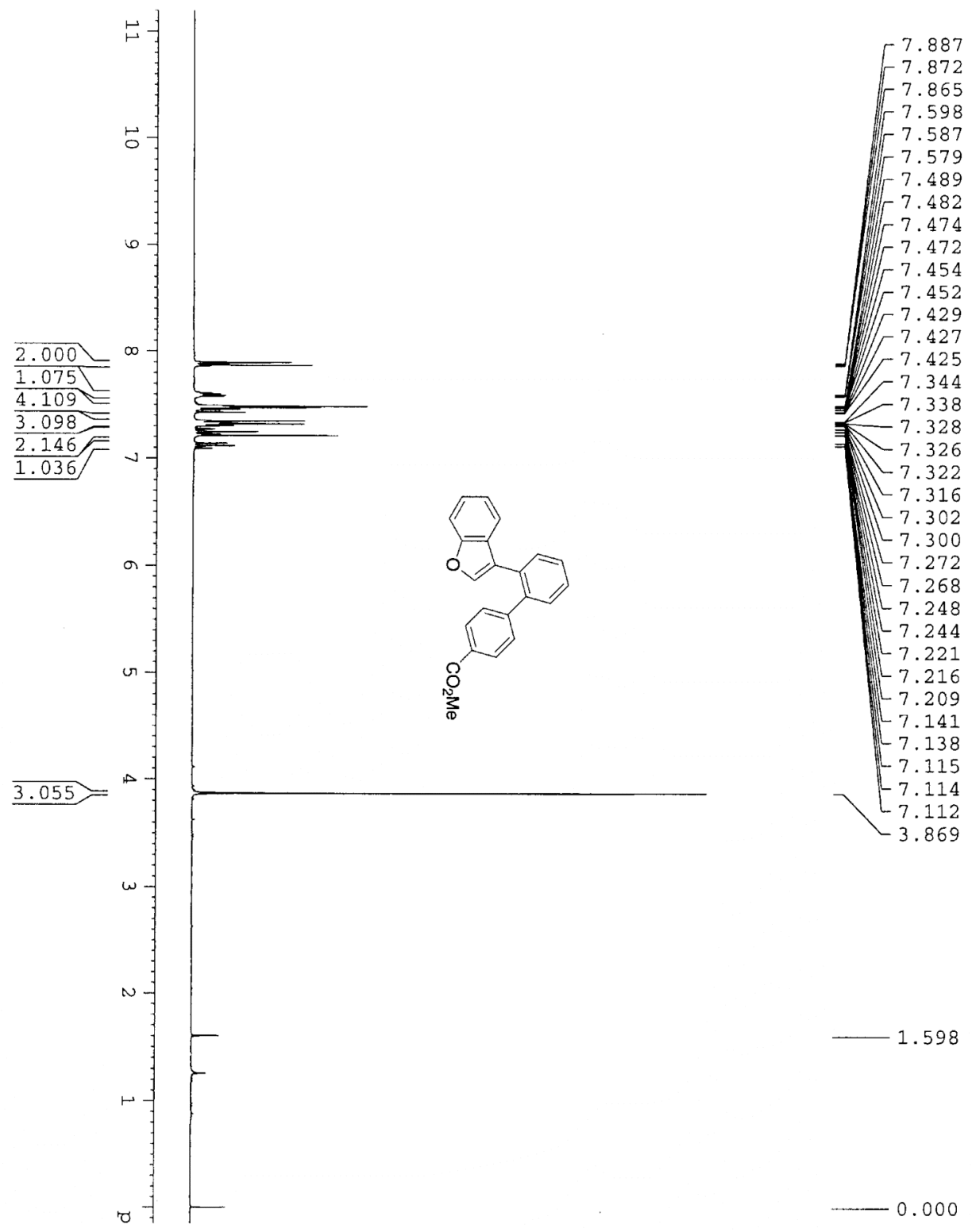

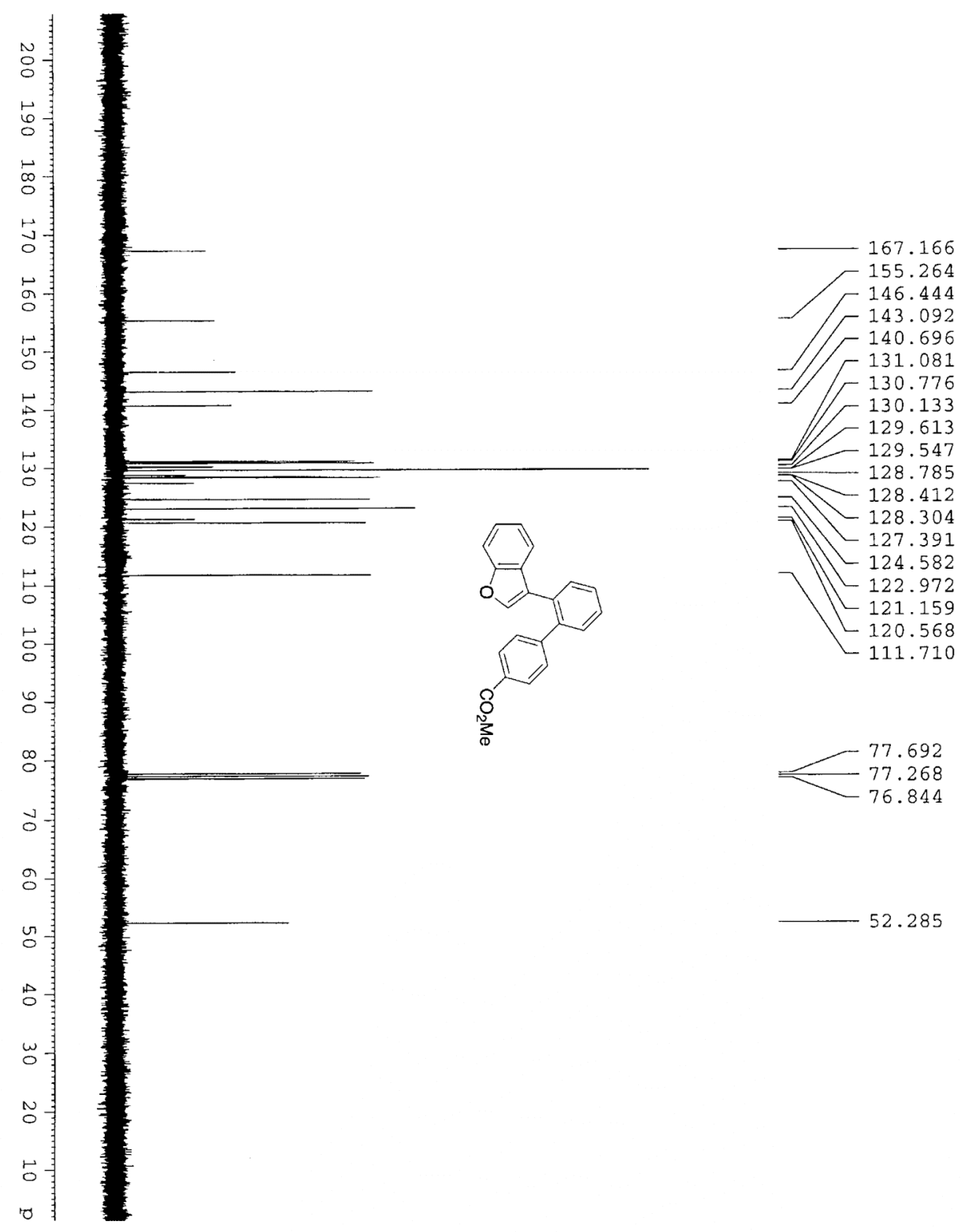

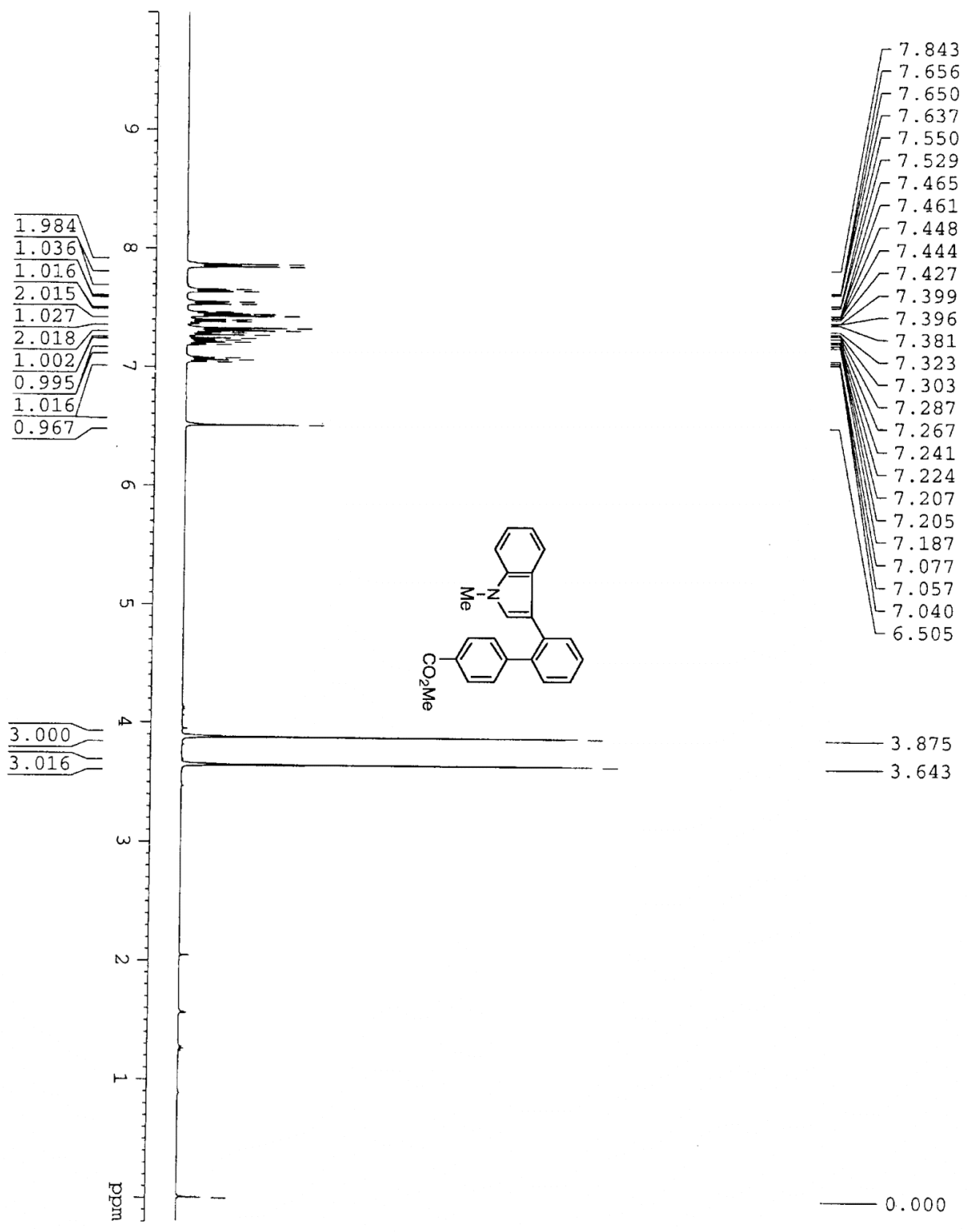

$-0.000$ 


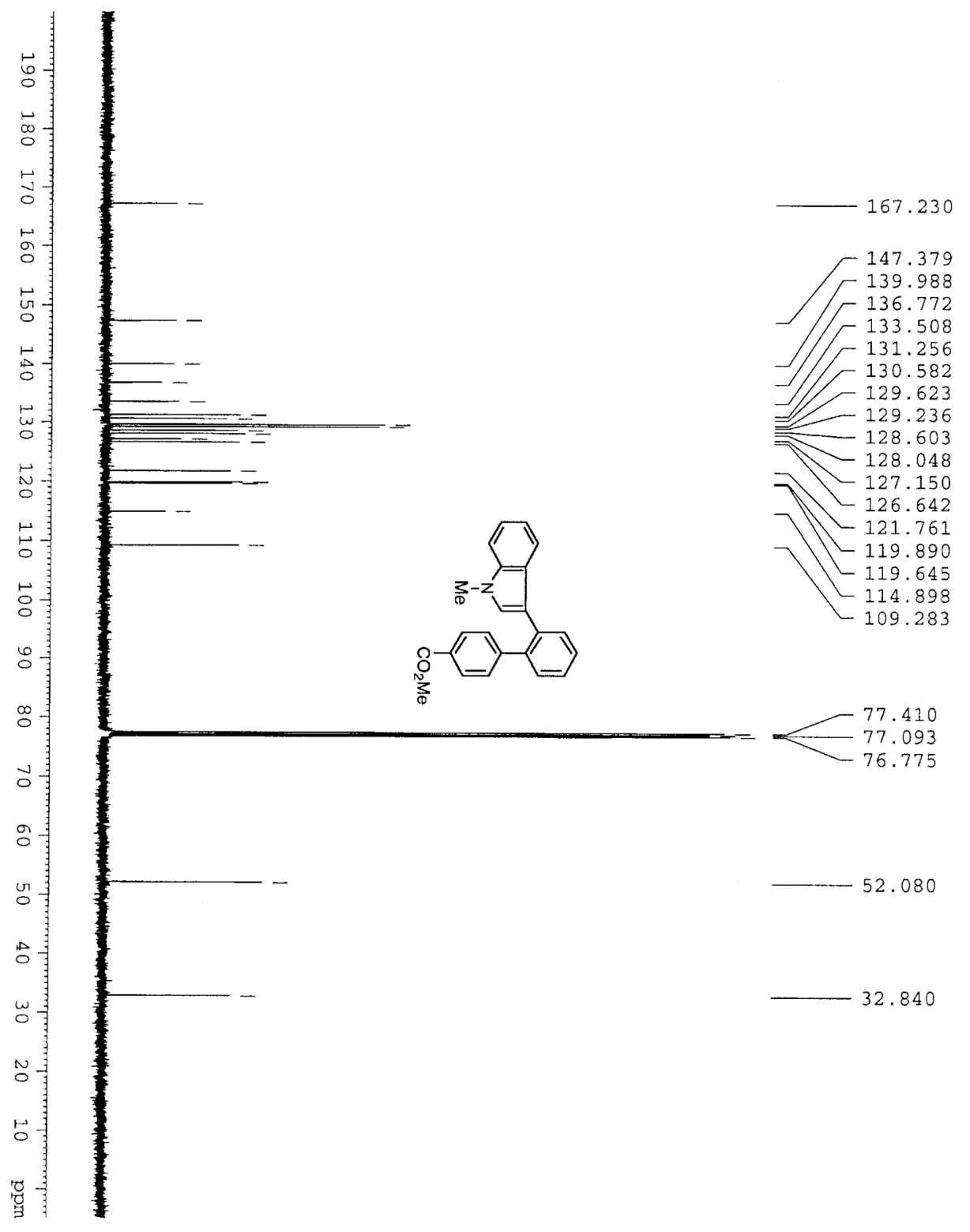




\section{References}

(1) (a) Campo, M. A.; Larock, R. C. Org. Lett. 2000, 2, 3675. (b) Campo, M. A.; Larock, R. C. J. Org. Chem. 2002, 67, 5616.

(2) Campo, M. A.; Larock, R. C. J. Am. Chem. Soc. 2002, 124, 14326.

(3) Ozasa, S.; Fujoka, Y.; Tsukada, M.; Ibuki, E. Chem. Pharm. Bull. 1981, 29, 344.

(4) Murray, M. M.; Kaszynski, P.; Kaisaki, D. A.; Dougherty, D. A. J. Am. Chem. Soc. 1994, $116,8152$.

(5) Ma, C.; Liu, X.; Li, X.; Flippen-Anderson, J.; Yu, S.; Cook, J. M. J. Org. Chem. 2001, 66, 4525 .

(6) Gibson, S. E.; Guillo, N.; Middleton, R. J.; Thuilliez, A.; Tozer, M. J. J. Chem. Soc., Perkin Trans. 1 1997, 447.

(7) Hart, H.; Harada, K.; Du, C. J. Org. Chem. 1985, 50, 3104.

(8) Karl, J.; Gust, R.; Spruss, T.; Schneider, M. R.; Schoenenberger, H.; Engel, J.; Wrobel, K. H.; Lux, F.; Haeberlin, S. T. J. Med. Chem. 1988, 31, 72.

(9) Wright, S. W.; Hageman, D. L.; McClure, L. D. J. Org. Chem. 1994, 59, 6095.

(10) Ibuki, E.; Ozasa, S.; Fujioka, Y.; Yanogihara, Y. Chem. Pharm. Bull. 1982, 30, 802.

(11) Trost, B. M.; Arndt, H. C. J. Am. Chem. Soc. 1973, 95, 5288.

(12) Hanson, G.; Kemp, D. S. J. Org. Chem. 1981, 46, 5441.

(13) Freeman, P. K.; Tafesh, A. M.; Clapp, G. E. J. Org. Chem. 1989, 54, 782. 